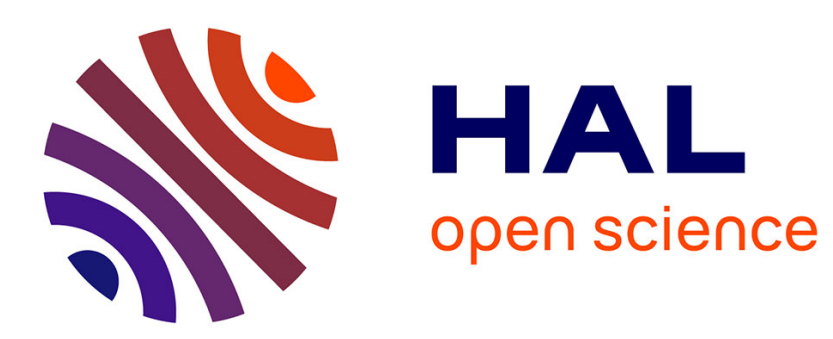

\title{
Asymmetrical three-dimensional travelling gravity waves
}

Gérard Iooss, Pavel Plotnikov

\section{To cite this version:}

Gérard Iooss, Pavel Plotnikov. Asymmetrical three-dimensional travelling gravity waves. Archive for Rational Mechanics and Analysis, 2011, 200 (3), pp.92. hal-01265172

\section{HAL Id: hal-01265172 \\ https://hal.univ-cotedazur.fr/hal-01265172}

Submitted on 31 Jan 2016

HAL is a multi-disciplinary open access archive for the deposit and dissemination of scientific research documents, whether they are published or not. The documents may come from teaching and research institutions in France or abroad, or from public or private research centers.
L'archive ouverte pluridisciplinaire HAL, est destinée au dépôt et à la diffusion de documents scientifiques de niveau recherche, publiés ou non, émanant des établissements d'enseignement et de recherche français ou étrangers, des laboratoires publics ou privés. 


\title{
Asymmetrical three-dimensional travelling gravity waves
}

\begin{abstract}
We consider periodic travelling gravity waves at the surface of an infinitely deep perfect fluid. The pattern is non symmetric with respect to the propagation direction of the waves and we consider a general non resonant situation. Defining a couple of amplitudes $\varepsilon_{1}, \varepsilon_{2}$ along the basis of wave vectors which satisfy the dispersion relation, first we give the formal asymptotic expansion of bifurcating solutions in powers of $\varepsilon_{1}, \varepsilon_{2}$. Then, introducing an additional equation for the unknown diffeomorphism of the torus, associated with an irrational rotation number, which allows to transform the differential at the successive points of the Newton iteration method, into a differential equation with two constant main coefficients, we are able to use a descent method leading to an invertible differential. Then by using an adapted Nash Moser theorem, we prove the existence of solutions with the above asymptotic expansion, for values of the couple $\left(\varepsilon_{1}^{2}, \varepsilon_{2}^{2}\right)$ in a subset of the first quadrant of the plane, with asymptotic full measure at the origin.
\end{abstract}

Key words: nonlinear water waves; small divisors; bifurcation theory; pseudodifferential operators; travelling gravity waves; asymmetric 3D waves; rotation number

AMS: 76B15; 47J15; 35S15; 76B07

Gérard Iooss

IUF, Université de Nice, Labo J.A.Dieudonné, 06108 Nice Cedex 02, France

E-mail: gerard.iooss@unice.fr

Pavel Plotnikov

Lavrentyev Institute of Hydrodynamics, Lavryentyev pr. 15, Novosibirsk 630090, Russia

E-mail: plotnikov@hydro.nsc.ru 


\section{Introduction}

\subsection{Presentation and history of the problem}

We consider small-amplitude three-dimensional doubly periodic travelling gravity waves on the free surface of a perfect fluid. These waves are steady in a frame moving with the velocity of the wave $(-\mathbf{c}$ in the absolute reference frame). The fluid layer is supposed to be infinitely deep, and the flow is irrotational only subjected to gravity. The bifurcation parameters are the horizontal phase velocity, and the direction of propagation of the travelling waves, the infinite depth case being not essentially different from the finite depth case.

In 1847 Stokes [27] gave a nonlinear theory of two-dimensional travelling gravity waves, computing the flow up to the cubic order of the amplitude of the waves, and the first mathematical proofs for such periodic two-dimensional waves are due to Nekrasov [22] and Levi-Civita [19]. Mathematical progress on the study of three-dimensional doubly periodic water waves came much later. In particular, first formal expansions in powers of the amplitude of three-dimensional travelling waves can be found in papers [7] and [26]. One can find many references and results of research on this subject in the review paper of Dias and Kharif [6]. Reeder and Shinbrot (1981)[24] proved the existence of gravity-capillary waves with symmetric diamond patterns, resulting from (horizontal) wave vectors belonging to a lattice $\Gamma^{\prime}$ (dual to the spatial lattice $\Gamma$ of the doubly periodic pattern) spanned by two wave vectors $K_{1}$ and $K_{2}$ with the same length, the velocity of the wave being in the direction of the bissectrix of these two wave vectors. This was completed by Craig and Nicholls (2000) [3] who used the hamiltonian formulation introduced by Zakharov [28], with a variational method. These waves appear in litterature as "short crested waves" (see Roberts and Schwartz [25], Bridges, Dias, Menasce [1] for an extensive discussion on various situations and numerical computations), and the fact that the surface tension is supposed not to be too small is essential for being able to use Lyapunov-Schmidt technique, and the authors mention a small divisor problem if there is no surface tension. Asymmetrical "simple" doubly periodic waves in the non resonant cases were also considered by Craig and Nicholls (2002) [2] who gave the principal part of the formal Taylor series, taking into account of the two-dimensions of the vector parameter c. They emphasize the fact that this expansion is only formal in the absence of surface tension.

Another type of mathematical results are obtained in using "spatial dynamics", in which one of the horizontal coordinates (the distinguished direction) plays the role of a time variable, as was initiated by Kirchgässner [17] and extensively applied to two-dimensional water wave problems (see a review in [5]). The advantage of this method is that one does not choose the behavior of the solutions in the direction of the distinguished coordinate, and solutions periodic in this coordinate are a particular case, as well as quasiperiodic or localized solutions (solitary waves). In this framework one may a priori assume periodicity in a direction transverse to the distinguished direction, and a periodic solution in the distinguished direction is automatically 
doubly periodic and non necessarily symmetric with respect to the propagation direction. The first mathematical results obtained by this method, containing 3-dimensional doubly periodic travelling waves, start with Haragus, Kirchgässner, Groves and Mielke (2001) [11], [9], [13], generalized by Groves and Haragus (2003) [10]. They use a hamiltonian formulation and center manifold reduction. This is essentially based on the fact that the spectrum of the linearized operator is discrete and has only a finite number of eigenvalues on the imaginary axis. These eigenvalues are related with the dispersion relation mentioned above. Here, one component (or multiples of such a component) of the wave vector $K$ is imposed in a direction transverse to the distinguished one, and there is no restriction for the component of $K$ in the distinguished direction, which, in solving the dispersion relation, gives the eigenvalues of the linearized operator on the imaginary axis. However, it appears that the number of imaginary eigenvalues becomes infinite when the surface tension cancels, which prevents the use of center manifold reduction in the limiting case we are considering in the present paper.

One essential difficulty here, with respect to the existing literature, except our previous work [16], is that we assume the absence of surface tension. Indeed the surface tension plays a major role in all existing proofs for threedimensional travelling gravity-capillary waves, and when the surface tension is very small, which is the case in many usual situations, this implies a reduced domain of validity of results strongly dependent on the existence of a non small surface tension. In our previous work [16] on three-dimensional travelling gravity waves, there is no surface tension, and we restricted the study to the existence of diamond waves: the periodic lattice is a diamond lattice, and there are equal amplitudes at the leading order for the two basic wave vectors $K_{1}, K_{2}$ symmetric with respect to the propagation direction of the waves. We proved the existence of bifurcating diamond gravity waves, symmetric with respect to the propagation direction of the waves. In this case, because of the absence of surface tension, a small divisor problem arises and since the use of Nash-Moser theorem is necessary, one of the essential technical ingredients for the preparation of the differential near the origin which needs to be inverted, is that the integral curves of the horizontal projection of the velocity field $V$ of particles, may be transformed by a suitable diffeomorphism of the torus, into straight lines, parallel to the direction of propagation. This diffeomorphism was given by the solution of a simple ordinary differential equation, thanks to the required symmetries of the solution.

In the present work, we consider asymmetrical travelling gravity waves, which implies that the basic wave vectors $K_{1}$ and $K_{2}$ have not the same length, and given the two amplitudes on the basic modes, the direction of propagation $\mathbf{u}$ of the waves is part of the unknown. We need to find a diffeomorphism for "preparing" the differential we have to invert, and this is now a serious new difficulty here, leading to the necessity to compute the rotation number $\rho$ of the velocity field $V$, (which was $\rho=1$ in the symmetric case) for which a diophantine condition is now necessary. This leads to our choice to consider as unknowns in our problem: the main unknowns (the velocity potential and the shape of the free surface) together with the unknown diffeomorphism and the associated rotation number of $V$. This allows 
us to use only once the Nash-Moser implicit function theorem. Finally we are able to prove the existence of asymmetrical gravity waves (see Theorem 2 ) for nearly all choices of angles $\theta_{1}, \theta_{2}$ made by the basis of non symmetric wave vectors $K_{1}, K_{2}$ with the direction $\mathbf{u}_{0}$ of the critical velocity, and for 2-dimensional parameter values $\left(\varepsilon_{1}^{2}, \varepsilon_{2}^{2}\right)$ in a set for which 0 is a Lebesgue point ( 0 corresponds to the critical value $\mathbf{c}_{c}$ of the velocity vector $\mathbf{c}$ ).

As a side result which is worthy to mention, we prove the existence of a "directional Stokes drift" (see Lemma 3). This means that the asymptotic direction of the trajectories of fluid particles on the free surface differs from the propagation direction of the waves by an angle of order $O\left(\varepsilon_{1}^{2}+\varepsilon_{2}^{2}\right)$. This angle (0 for diamond waves) cancels for an appropriate choice of the ratio between amplitudes $\varepsilon_{1}, \varepsilon_{2}$.

Finally, nice experimental results are known on diamond waves (symmetric with respect to the propagation direction, see Hammack, Henderson, Segur [12]). We have now the explicit expansion in powers of the two amplitudes of asymmetric waves, and the directional Stokes drift property, it would be then very interesting to have experiments allowing to verify the domain of validity of these formulas.

\subsection{Formulation of the problem}

We are looking for waves travelling with velocity $-\mathbf{c}$, so we consider the system in the moving frame where the waves look steady. Assume that a flow occupies a domain in the Euclidian space of points $x=\left(x_{1}, x_{2}, x_{3}\right)$ bounded from above by a free surface $\Sigma:\left\{x_{3}=\eta\left(x_{1}, x_{2}\right)\right\}$. Let us denote by $\varphi$ the potential defined by $\varphi=\phi-\mathbf{c} \cdot X$, where $\phi$ is the usual velocity potential in the moving frame (the rest state is then $\varphi=0), X=\left(x_{1}, x_{2}\right)$ is the 2-dim horizontal coordinate, $x_{3}$ is the vertical coordinate. We choose $|\mathbf{c}|$ for the velocity scale, and $L$ for a length scale (to be chosen below), and we still denote by $\left(X, x_{3}\right)$ the new coordinates, and by $\varphi, \eta$ the unknown functions. Now define the inverse squared Froude number by $\mu=g L / c^{2}$, where $g$ denotes the acceleration of gravity, and by $\mathbf{u}$ the unit vector in the direction of $\mathbf{c}$. Following V.E.Zakharov we introduce the new unknown function $\psi(X)=\varphi(X, \eta(X))$, and define the Dirichlet-Neumann operator $\mathcal{G}_{\eta}$ by

$$
\mathcal{G}_{\eta} \psi=\left.\sqrt{1+(\nabla \eta)^{2}} \frac{d \varphi}{d n}\right|_{x_{3}=\eta(X)}=\left.\frac{\partial \varphi}{\partial x_{3}}\right|_{x_{3}=\eta(X)}-\nabla \eta \cdot \nabla_{X} \varphi,
$$

where $n$ is normal to $\Sigma$, exterior to the domain of the flow, and $\varphi$ is the bounded solution of the $\eta$ - dependent Dirichlet problem

$$
\Delta \varphi=0 \text { for } x_{3}<\eta(X), \quad \varphi=\psi \text { for } x_{3}=\eta(X) .
$$

This definition of $\mathcal{G}_{\eta}$ follows [18] and insures the selfadjointness and positivity of this linear operator. Then the problem of a spatial gravity waves is equivalent, see [16] for example, to the system of nonlinear operator equations for function $U=(\psi, \eta)$ and parameters $\mathbf{u}$ and $\mu$,

$$
\mathcal{F}(U, \mu, \mathbf{u})=0, \text { where } \mathcal{F}=\left(\mathcal{F}_{1}, \mathcal{F}_{2}\right),
$$




$$
\begin{aligned}
\mathcal{F}_{1}(U, \mathbf{u})= & : \mathcal{G}_{\eta}(\psi)-\mathbf{u} \cdot \nabla_{X} \eta \\
\mathcal{F}_{2}(U, \mu, \mathbf{u})= & : \mathbf{u} \cdot \nabla_{X} \psi+\mu \eta+\frac{(\nabla \psi)^{2}}{2}+ \\
& -\frac{1}{2\left(1+\left(\nabla_{X} \eta\right)^{2}\right)}\left\{\nabla_{X} \eta \cdot\left(\nabla_{X} \psi+\mathbf{u}\right)\right\}^{2} .
\end{aligned}
$$

We specialize our study to spatially periodic 3-dimensional travelling waves, i.e. solutions $\eta$ and $\varphi$ are periodic in $X$. This means that there are two independent wave vectors $K_{1}, K_{2} \in \mathbb{R}^{2}$ generating a lattice

$$
\Gamma^{\prime}=\left\{\mathbf{k}=n_{1} K_{1}+n_{2} K_{2}: n_{j} \in \mathbb{Z}, j=1,2\right\},
$$

and a dual lattice $\Gamma$ of periods in $\mathbb{R}^{2}$ such that

$$
\Gamma=\left\{\boldsymbol{\lambda}=m_{1} \boldsymbol{\lambda}_{1}+m_{2} \boldsymbol{\lambda}_{2}: m_{j} \in \mathbb{Z}, \boldsymbol{\lambda}_{j} \cdot K_{l}=2 \pi \delta_{j l}, \quad j, l=1,2\right\} .
$$

The Fourier expansions of $\eta$ and $\varphi$ are in terms of $e^{i \mathbf{k} \cdot X}$, where $\mathbf{k} \in \Gamma^{\prime}$ and $\mathbf{k} \cdot \boldsymbol{\lambda}=2 n \pi, n \in \mathbb{Z}$, for $\boldsymbol{\lambda} \in \Gamma$. The situation we consider in the further analysis, is with a lattice $\Gamma^{\prime}$ generated by the wave vectors

$$
K_{1}=\left(1, \tau_{1}\right), K_{2}=\lambda\left(1,-\tau_{2}\right) .
$$

This means that we have chosen the length scale $L$ for having 1 in the first component of $K_{1}$, and $\tau_{1}, \tau_{2}$ measure the angles between the wave vectors $K_{1}, K_{2}$ and the $x_{1}$ axis. We define the Fourier coefficients of a periodic function $u$ on the lattice $\Gamma$ by

$$
\widehat{u}_{\mathbf{k}}=|\Omega|^{-1 / 2} \int_{\Omega} u(X) \exp (-i \mathbf{k} \cdot X) d X, \quad|\Omega|=4 \pi^{2}\left\{\lambda\left(\tau_{1}+\tau_{2}\right)\right\}^{-1}
$$

where $\Omega$ is the paralellogram built with $\boldsymbol{\lambda}_{1}, \boldsymbol{\lambda}_{2}$. For $m \geq 0$ we denote by $H^{m}\left(\mathbb{R}^{2} / \Gamma\right)$ the Sobolev space of periodic functions of $X \in \mathbb{R}^{2} / \Gamma$ which are square integrable on a period, with their partial derivatives up to order $m$, and we can choose the norm as

$$
\|u\|_{m}=\left\{\sum_{\mathbf{k} \in \Gamma^{\prime}}(1+|\mathbf{k}|)^{2 m}\left|\widehat{u}_{\mathbf{k}}\right|^{2}\right\}^{1 / 2} .
$$

For every $s \geq 0$ we denote by $H^{s}$ the Hilbert space of all $2 \pi$ periodic functions $u: \mathbb{R}^{2} \rightarrow \mathbb{R}$ having the finite norm

$$
\|u\|_{s}=\left\{\sum_{\mathbf{k} \in \mathbb{Z}^{2}}\left(1+|\mathbf{k}|^{2}\right)^{s}\left|\hat{u}_{\mathbf{k}}\right|^{2}\right\}^{1 / 2}, \text { where } \widehat{u}_{\mathbf{k}}=\frac{1}{2 \pi} \int_{\mathbb{R}^{2} /(2 \pi \mathbb{Z})^{2}} e^{-i \mathbf{k} \cdot Y} u(Y) d Y .
$$

Denote also by $H_{e}^{s}$ the closed subspace of $H^{s}$ which consists of all even functions $u \in H^{s}$, i.e. $u(Y)=u(-Y)$, and by $H_{o}^{s}$ the subspace of the odd functions $(u(-Y)=-u(Y))$. We also denote by $H^{s, 0}$ the subspace of $H^{s}$ which consists of all function orthogonal to 1, i.e., $H^{s, 0}=\left\{u \in H^{s}: \hat{u}_{0}=0\right\}$. Let us define the 2-components function space

$$
\mathbb{H}^{m}\left(\mathbb{R}^{2} / \Gamma\right)=H_{0}^{m}\left(\mathbb{R}^{2} / \Gamma\right) \times H^{m}\left(\mathbb{R}^{2} / \Gamma\right) \text { with } \quad\|U\|_{m}=\|\psi\|_{H^{m}}+\|\eta\|_{H^{m}},
$$


where $H_{0}^{m}$ means functions with 0 average, and $U=(\psi, \eta)$. The 0 average condition comes from the fact that the value $\psi$ of the potential is defined up to an additive constant (easily checked in equations (1.3), (1.4)). Moreover, the average of the right hand side of (1.3) is 0 as it can be easily checked (this is proved for instance in [2]). We have the following

$\mathrm{F}(\mathrm{U}, \mathrm{mu}) \quad$ Lemma 1 For any fixed $m \geq 3$, the mapping

$$
(U, \mu, \mathbf{u}) \mapsto \mathcal{F}(U, \mu, \mathbf{u}) \quad \text { is } C^{\infty}: \mathbb{H}^{m}\left(\mathbb{R}^{2} / \Gamma\right) \times \mathbb{R} \times \mathbb{S}_{1} \rightarrow \mathbb{H}^{m-1}\left(\mathbb{R}^{2} / \Gamma\right)
$$

in the neighborhood of $\{0\} \times \mathbb{R} \times \mathbb{S}_{1}$. The mapping $\mathcal{F}(\cdot, \mu, \mathbf{u})$ is equivariant under translations of the plane:

$$
\mathcal{T}_{\mathbf{v}} \mathcal{F}(U, \mu, \mathbf{u})=\mathcal{F}\left(\mathcal{T}_{\mathbf{v}} U, \mu, \mathbf{u}\right) \text { where } \mathcal{T}_{\mathbf{v}} U(X)=U(X+\mathbf{v}),
$$

and it is also equivariant with respect of the symmetry $\mathcal{S}_{0}$ defined by the following representation of the symmetry with respect to 0

$$
\left(\mathcal{S}_{0} U\right)(X)=(-\psi(-X), \eta(-X)) .
$$

In addition, there is $M_{3}>0$, such that for $\|U\|_{3} \leq M_{3}$ and $|\mu| \leq M_{3}, \mathcal{F}$ satisfies for any $m \geq 3$ the "tame" estimate

$$
\|\mathcal{F}(U, \mu, \mathbf{u})\|_{m-1} \leq c_{m}\left(M_{3}\right)\|U\|_{m} .
$$

Proof The $C^{\infty}$ smoothness of $(\psi, \eta) \mapsto \mathcal{G}_{\eta}(\psi): \mathbb{H}^{m}\left(\mathbb{R}^{2} / \Gamma\right) \rightarrow H^{m-1}\left(\mathbb{R}^{2} / \Gamma\right)$ comes from the study of the Dirichlet-Neumann operator, and the properties of elliptic operators. This regularity is proved in particular by Craig et al in [4], Craig and Nicholls in [3], D.Lannes in [18], Hu and Nicholls in [14], and by Buffoni et al in [8] (the most elementary proof in Sobolev spaces). Notice that $H^{s}\left(\mathbb{R}^{2} / \Gamma\right)$ is an algebra for $s>1$. We choose here to work with Sobolev spaces, with $(\psi, \eta) \in \mathbb{H}^{m}\left(\mathbb{R}^{2} / \Gamma\right)$ and we refer to [18] for the proof of the "tame" estimate, valid for any $m \geq 3$ (here simpler than in [18] since we have periodic functions and there is no bottom wall). The equivariance of $\mathcal{F}$ under translations of the plane is obvious. The system $(1.2,1.3,1.4)$ is equivariant under $\mathcal{S}_{0}$, as this results from the reversibility of the original Euler system.

It is important to note that for any $\mu \in \mathbb{R}$, the operator equation (1.2) has the trivial solution $U=0, \mathbf{u}_{0}=(1,0)$ and our main goal is to find non-trivial solutions which bifurcate from the critical value $\mu=\mu_{c}$, where $\boldsymbol{\tau}=\left(\tau_{1}, \tau_{2}\right)$. Moreover, we are looking for non-trivial solutions satisfying a special topological condition.

The specificity of spatial water wave problems is that the free boundary is a two dimensional surface, then the liquid particles moving along the surface, have two degrees of freedom, and the totality of their trajectories forms a flow on the periodic free boundary. As it was shown in [16] this flow is the geodesic flow for the Jacobi metric induced by the gravity field. Recall that in our notation each trajectory $x=x(t)$ is determined by the relation $x(t)=\{X(t), \eta(X(t))\}$ in which $X(t)$ is a solution of the equation

$$
\dot{X}=V(X), \quad-\infty<t<\infty,
$$


where the periodic vector field $V=\mathbf{u}_{0}+O(\varepsilon)$ determined by (4.1) is the horizontal projection of the fluid velocity restricted to the free surface. Notice that the behavior of integral lines of the perturbed vector field $V$ may be very complicated, which makes impossible the analysis of the problem, since these lines serve as characteristics of the linearized equations. Hence we have to impose restrictions on the behavior of solutions to (1.6). In order to do this we recall the notion of rotation number of a solution to this equation.

Definition 1 We say that a solution $X(t)$ of equation (1.6) has a rotation number $\rho$ with respect to the lattice $\Gamma$ if

$$
\sup _{t} \operatorname{dist}\left\{X(t), \mathbf{l}_{\rho}\right\}<\infty, \quad \inf _{t}\left(\boldsymbol{\lambda}_{1}+\rho \boldsymbol{\lambda}_{\mathbf{2}}\right) \cdot V(X(t))>0,
$$

where $\mathbf{l}_{\rho}$ is the straight line parallel to the vector $\boldsymbol{\lambda}_{1}+\rho \boldsymbol{\lambda}_{2}$. We say that equation (1.6) determines a foliation of the torus $\mathbb{R}^{2} / \Gamma$ with rotation number $\rho$, if each solution of (1.6) has the rotation number $\rho$.

For the trivial solution, the integral curves of $V=\mathbf{u}_{0}$ are horizontal straight lines on the $X$-plane. They form a trivial foliation of the torus $\mathbb{R}^{2} / \Gamma$ with the rotation number $\rho_{c}=\lambda$.

Hypothesis 1 Further we are looking for solutions of the asymmetrical water waves problem satisfying an extra topological condition: the integral curves of the vector field $V$ form a foliation of the torus $\mathbb{R}^{2} / \Gamma$ with rotation number $\rho$ close to $\rho_{c}$. In this setting $\rho$ is unknown and is an integral part of the solution.

The existence of such a foliation is equivalent to the existence of a foliation of the Jacobi geodesic flow on the free surface [16]. A distinction needs to be drawn between the question on the existence of a single geodesic with a given rotation number and the question on the existence of a geodesic foliation. In the first case the answer is positive and the corresponding result holds true even in multidimensional case, see [20], [23]. The common belief is that in generic cases the geodesic foliation does not exist because of the occurrence of gaps related as Aubry-Maser sets. Nevertheless, as it was shown in [21], the trivial foliation is stable with respect to perturbations of $V$ if the rotation number $\rho_{c}$ is irrational and satisfies a Diophantine condition. Following this result we restrict our considerations to the case of irrational $\rho$.

It is worthy to notice that for symmetric diamond waves, the rotation number of trajectories of liquid particles on the free surface is equal to 1 . In this very special case the corresponding foliation exists only because of extra symmetric properties of diamond waves, which do not hold for asymmetric waves.

\subsection{Method and Results}

We now formulate the main results proved in this paper, on the existence of asymmetric travelling gravity waves satisfying equations (1.2), (1.3), (1.4). We find a unique solution in a small neighborhood of an approximate solution $U_{N}(\varepsilon), \mu_{N}(\varepsilon), \mathbf{u}_{N}(\varepsilon)$ which is chosen such that $\psi$ is odd, and $\eta$ even in 
$X$. Here the parameter $\varepsilon=\left(\varepsilon_{1}, \varepsilon_{2}\right)$ represents the (small) two-dimensional amplitude of the waves. From a single solution of this type, we then deduce a torus family of solutions by using the translation operator $\mathcal{T}_{\mathbf{v}}$. We first define the subspace where $U$ lies:

$\mathbb{H}_{(S)}^{k}\left(\mathbb{R}^{2} / \Gamma\right)=\left\{U=(\psi, \eta) \in \mathbb{H}^{k}\left(\mathbb{R}^{2} / \Gamma\right): \psi(-X)=-\psi(X), \eta(-X)=\eta(X)\right\}$.

Then we prove the following

Lemma 2 For any fixed integer $N \geq 3$, and $\boldsymbol{\tau}=\left(\tau_{1}, \tau_{2}\right) \in \mathbb{R}^{+2} \backslash\{0\}$, we define

$$
\lambda=\left(1+\tau_{2}^{2}\right)^{1 / 2}\left(1+\tau_{1}^{2}\right)^{-1 / 2}, \quad \mu_{c}=\left(1+\tau_{1}^{2}\right)^{-1 / 2},
$$

and assume (non resonance condition) that the equation $\mu_{c}|\mathbf{k}|=\left(\mathbf{k} \cdot \mathbf{u}_{0}\right)^{2}$, where $\mathbf{u}_{0}=(1,0)$, has only the solutions $\mathbf{k}=\mathbf{0}, \pm K_{1}, \pm K_{2}$, where $K_{1}=$ $\left(1, \tau_{1}\right), K_{2}=\lambda\left(1,-\tau_{2}\right)$, and $\mathbf{k} \in \Gamma^{\prime}$. Then, for any $\varepsilon=\left(\varepsilon_{1}, \varepsilon_{2}\right) \in \mathbb{R}^{+2}$, there is an approximate asymmetric travelling wave given by the expansion

$$
\begin{gathered}
U_{N}(\varepsilon)=\sum_{p+q=1}^{N} \varepsilon_{1}^{p} \varepsilon_{2}^{q} U_{p q} \in \mathbb{H}_{(S)}^{k}\left(\mathbb{R}^{2} / \Gamma\right), \text { for any } k \geq 3, \\
\mu_{N}(\varepsilon)=\mu_{c}+\sum_{p+q=1}^{N / 2} \varepsilon_{1}^{2 p} \varepsilon_{2}^{2 q} \mu_{p q}, \mathbf{u}_{N}(\varepsilon)=\mathbf{u}_{0}+\sum_{p+q=1}^{N / 2} \varepsilon_{1}^{2 p} \varepsilon_{2}^{2 q} \mathbf{u}_{p q},
\end{gathered}
$$

where $\mu_{N}(\varepsilon) \in \mathbb{R}^{+}, \mathbf{u}_{N}(\varepsilon) \in \mathbb{S}_{1}$. In addition, we have

$$
\mathcal{F}\left(U_{N}(\varepsilon), \mu_{N}(\varepsilon), \mathbf{u}_{N}(\varepsilon)\right)=|\varepsilon|^{N+1} \mathcal{Q}(\varepsilon),
$$

with $\mathcal{Q}(\varepsilon)$ bounded in $\mathbb{H}_{(S)}^{k}\left(\mathbb{R}^{2} / \Gamma\right)$, uniformly in $\varepsilon$.

Proof This Lemma follows directly from Theorem 4.

We notice that the non resonance condition is in fact a condition on $\left(\tau_{1}, \tau_{2}\right)$. It is indeed satisfied for a full measure set in $\mathbb{R}^{+2}$ as it results from Theorem 10. Notice also the difference between this result and the corresponding one in [16] where only one amplitude occurs. In the above Lemma, if we make $\tau_{1}=\tau_{2}$, and $\varepsilon_{1}=\varepsilon_{2}$ we recover the result of Lemma 1.2 in [16].

The following theorem is the main result of the paper

existenceThm Theorem 2 Let us choose arbitrary integers $l \geq 34, N$ even $\geq 4$ and a real number $0<\delta<1$. Then, there is a full measure subset $\mathcal{T}$ of $\overline{\mathbb{R}}^{+2}$ such that for any $\boldsymbol{\tau} \in \mathcal{T}$ there exists a subset $\mathcal{E}(\boldsymbol{\tau})$ of the quadrant $\left\{\left(\varepsilon_{1}^{2}, \varepsilon_{2}^{2}\right) \in \mathbb{R}^{+2}\right\}$ for which 0 is a Lebesgue point, i.e.

$$
\frac{2}{\epsilon^{2}} \operatorname{meas}\left(\mathcal{E}(\boldsymbol{\tau}) \cap\left\{\varepsilon_{1}^{2}+\varepsilon_{2}^{2}<\epsilon\right\}\right) \rightarrow 1 \text { as } \epsilon \rightarrow 0 .
$$

Moreover, for $\delta<\varepsilon_{1} / \varepsilon_{2}<1 / \delta$ and $\varepsilon=\left(\varepsilon_{1}, \varepsilon_{2}\right) \in \mathcal{E}(\boldsymbol{\tau})$, the system (1.2, 1.3, 1.4) has a unique solution of the form

$$
\begin{aligned}
U & =U_{2 N}(\varepsilon)+|\varepsilon|^{N} \breve{U}(\varepsilon) \in \mathbb{H}_{(S)}^{l}\left(\mathbb{R}^{2} / \Gamma\right), \\
\mu & =\mu_{2 N}(\varepsilon)+|\varepsilon|^{N} \breve{\mu}(\varepsilon) \in \mathbb{R}^{+}, \\
\mathbf{u} & =\mathbf{u}_{2 N}(\varepsilon)+|\varepsilon|^{N} \breve{\mathbf{u}}(\varepsilon) \in \mathbb{S}_{1},
\end{aligned}
$$

where $(\breve{U}(\varepsilon), \breve{\mu}(\varepsilon), \breve{\mathbf{u}}(\varepsilon)) \in \mathbb{H}_{(S)}^{l}\left(\mathbb{R}^{2} / \Gamma\right) \times \mathbb{R} \times \mathbb{R}^{2}$. 
The proof of this theorem follows the same structure as for the proof of the main theorem in [15] and in [16]. As in [16], the above result expressed in terms of bifurcation analysis, provides a branch of solutions bifurcating from a non isolated eigenvalue 0 in the spectrum of the linearized operator at the origin. However, there is an additional difficulty here, due to asymmetry.

After a simple study of the linearized system at the origin, made in section 2, we prove Theorem 4 in section 3, equivalent to Lemma 2 showing formally how to handle the pair of "angular parameters" $\tau_{j}$ and the pair of "amplitude parameters" $\varepsilon_{j}$. The formal computation used for proving this Theorem shows a small divisor problem, which prevents us to use the Lyapunov-Schmidt method, contrary to the case when there is surface tension and where this method would be possible to be used. We are then led to use the Nash-Moser implicit function theorem.

In section 4 we consider the linear operator $\mathcal{L}_{\mu, \mathbf{u}}(U)$ corresponding to the differential of $\mathcal{F}$ at a non zero point in $\mathbb{H}_{(S)}^{k}$, which we need to invert. The principal part of this operator is the sum of a second order derivative in one direction $V$ and of the Dirichlet-Neumann operator which is integrodifferential of first order, both parts depending periodically on $X$. The problem of invertibility of the operator $\mathcal{L}_{\mu, \mathbf{u}}(U)$ is equivalent to the problem of existence and uniqueness of periodic solutions to the linear pseudodifferential equation

$$
-\mathcal{J}^{*}\left(\frac{1}{\mathfrak{a}} \mathcal{J}(\delta \phi)\right)+\mathcal{G}_{\eta}(\delta \phi)=h,
$$

where $\mathcal{J}=V \cdot \nabla_{X}$ and $\mathcal{G}_{\eta}$ is the Dirichlet-Neumann operator. The coefficients of this equations depend on $U, \mu, \mathbf{u}$ and are determined by formulae (4.1) and (4.5). If we prove the solvability of this equation in the space $H^{k}\left(\mathbb{R}^{2} / \Gamma\right)$, then the existence of a solution to the nonlinear problem can be obtained by using a Nash-Moser iteration scheme. The main difficulty is that this equation has variable coefficients. Hence the first task is to find a diffeomorphism $Y=Y(X): \mathbb{R}^{2} / \Gamma \rightarrow \mathbb{R}^{2} /(2 \pi \mathbb{Z})^{2}$ of the torus which transforms the principal part into a constant operator for the two main derivatives. It is clear that this diffeomorphism must take the vector field $V_{1} \partial_{x_{1}}+V_{2} \partial_{x_{2}}$ to the vector field $\partial_{y_{1}}+\rho \partial_{y_{2}}$, where $\rho$ is the rotation number of the flow generated by $V$. It follows from this that the inverse mapping $X=X(Y): \mathbb{R}^{2} /(2 \pi \mathbb{Z})^{2} \rightarrow \mathbb{R}^{2} / \Gamma$ must satisfy the equation

$$
\mathcal{D} X \equiv \partial_{y_{1}} X+\rho \partial_{y_{2}} X=c(Y) V(X(Y)),
$$

where $c$ is a positive $2 \pi$-periodic function which will be specified below. For technical reasons it is convenient to take it in the form $c=(\sqrt{\nu} f)^{-1}$. After the change of variables in (1.9) we obtain (see section 5 ) the equation for the function $u(Y)=\delta \phi(X(Y))$

$$
\nu \mathcal{D}^{2} u+p \mathcal{D} u+\mathfrak{G}_{1} u+\mathfrak{G}^{*} u=\mathfrak{g} h(X(Y))
$$

where the differential operator $\mathcal{D}=\partial_{y_{1}}+\rho \partial_{y_{2}}$, the coefficients $\mathfrak{g}, p$ depend only on $U, \nu, \mu, \mathbf{u}$, and $\mathfrak{G}_{1}$ is a first order pseudodifferential operator with the symbol

$$
\left\{G_{1,22} k_{2}^{2}+2 G_{1,12} k_{2}\left(k_{1}+\rho k_{2}\right)+G_{1,12}\left(k_{1}+\rho k_{2}\right)^{2}\right\}^{1 / 2},
$$


and $\mathfrak{G}^{*}$ is a linear operator of order 0 . Hence the resulting equation has a parabolic character with leading derivatives $\mathcal{D}^{2}$ and $\partial_{y_{2}}$. In order to make constant the coefficients of these derivatives, we choose the function $f$ in such a way that $G_{1,22}=1$. As it is shown in section 5 , this gives the following equation for the diffeomorphism $X(Y)$

$$
\mathcal{F}_{3}(U, X, \rho, \nu, \mu, \mathbf{u}):=\mathcal{D} X-\left|\operatorname{det} X^{\prime}\right|^{1 / 3} \mathbf{U}(X(Y))=0,
$$

where

$\mathbf{U}(X)=\mathbf{F}[U, \nu, \mu, \mathbf{u}](X), \mathbf{F}[U, \nu, \mu, \mathbf{u}]=(\nu \mathfrak{a})^{-1 / 3}\left(V^{2}+(V \cdot \nabla \eta)^{2}\right)^{-1 / 6} V$,

$\rho$ and $\nu$ being unknown constants, necessary here for the solvability of (1.12), $\mathfrak{a}$ and $V$ are periodic functions of $X$ (depending on $U(X), \mu, \mathbf{u}$ ). We are looking for a solution in the form $X=\mathbb{T}^{-1} Y+\mathcal{W}(Y)$, where the matrix $\mathbb{T}^{-1}$ is formed with columns $\boldsymbol{\lambda}_{i} / 2 \pi, i=1,2$, and $\mathcal{W}$ is an unknown $2 \pi$ periodic function. We prove (Lemma 5 ) that (1.12) may be formally solved with respect to $(\mathcal{W}, \rho, \nu)$ in powers of $\varepsilon$, where at the origin $\rho=\lambda, \nu=$ $\left[\mu_{c} \lambda\left(\tau_{1}+\tau_{2}\right)\right]^{-1}$, provided that $\lambda \notin \mathbb{Q}$ (which restricts the choice of $\boldsymbol{\tau}=$ $\left.\left(\tau_{1}, \tau_{2}\right)\right)$.

The solving of (1.12) is the only first step in the study of the linear equation (1.9). In the course of application of the Nash-Moser scheme to the nonlinear system (1.2) we have to repeat this step infinitely many times. This makes the problem very difficult. The next step is of strategic importance. We add to system (1.2), the equation (1.12) for $X(Y), \rho, \nu$ and replace (1.2) by the extended system of operator equations

$$
\tilde{\mathcal{F}}(U, X, \rho, \nu, \mu, \mathbf{u})=0, \quad \tilde{\mathcal{F}}=\left(\mathcal{F}_{1}, \mathcal{F}_{2}, \mathcal{F}_{3}\right),
$$

where $\mathcal{F}_{1}$ and $\mathcal{F}_{2}$ are defined by (1.3), (1.4), and $\mathcal{F}_{3}$ is defined by (1.12).

We find again for this extended system an approximate solution under the form of a power series up to order $|\varepsilon|^{2 N}$, and this constitutes the starting point for the Newton iteration used in the Nash-Moser theorem. In particular we obtain $\rho=\rho_{2 N}(\varepsilon), \nu=\nu_{2 N}(\varepsilon)$, where $\rho_{2 N}$ and $\nu_{2 N}$ are polynomials of degree $N$ of variables $\varepsilon_{i}^{2}$. Next we fix $N$ and will consider $\rho=\rho_{2 N}$ and $\nu=\nu_{2 N}$ as given functions of the small parameter $\varepsilon$. In this framework, relations (1.13) form a system of operator equations for the function $U$, the diffeomorphism $X$, and parameters $\mu, \mathbf{u}$.

Section 7 studies the differential of the extended system with respect to $(U, X, \mu, \mathbf{u})$, which means that we consider, now for convenience, that the scalars $(\rho, \nu)$ are the bifurcation parameters. The last equation in (1.13) is independent of the first two, and this system has a triangular form. Therefore, in order to invert the derivative of the operator $\tilde{\mathcal{F}}$ we have to invert separately the linear operator $\mathcal{L}_{\mu, \mathbf{u}}$ corresponding to the differential of $\mathcal{F}$, and the differential $D_{X} \mathcal{F}_{3}$. The last question merits a detailed consideration here.

Equation (1.12) is a fully nonlinear system of PDE of indefinite type. However we claim that it belongs to the class of conjugacy problems and the invertibility of its linearization can be obtained by using Fourier analysis. In KAM theory the notion of conjugacy problems refers to a class of 
nonlinear equations in the form $\Phi(X, \mathbf{U})=0$ with a property $\Phi(X \circ Z, \mathbf{F}) \equiv$ $\Phi(Z, \Phi(X, \mathbf{F}))$. It is easy to see that the operator $\Phi=X^{\prime-1}\left|X^{\prime}\right|{ }^{\alpha} \mathbf{U} \circ X$ has this property and equation (1.12) can be regarded as a new example of conjugacy problem. In Section 7 we show that the linearized equation $D_{X} \mathcal{F}_{3}[X] \delta \phi=g$ can be reduced to the equation

$$
\mathcal{D} v-\frac{1}{3}(\operatorname{div} v) \varrho=X^{\prime-1} g+O\left(\mathcal{F}_{3}\right) v,
$$

introducteq2

where $\varrho=(1, \rho), \delta \phi=X^{\prime} v$. In Section 7 we study the structure of the extended equation in details, and reduce the inversion of the differential $D \tilde{\mathcal{F}}$ to a system of truncated equation (7.17)-(7.19). In its turn the truncated problem can be reduced to the system of decoupled equations (1.11)-(1.14). Since an approximate (with accuracy up to the discrepancy) solution to (1.14) can be derived by the Fourier method, our study focuses on equation (1.11).

Notice that the leading order terms in this equation have constant coefficients (this was the purpose in adding equation (1.12)), provided that the rotation number $\rho$ satisfies a suitable diophantine condition, which restricts the choice of amplitudes $\left(\varepsilon_{1}^{2}, \varepsilon_{2}^{2}\right)$ since we now consider $(\rho, \nu)=\rho_{2 N}(\varepsilon), \nu_{2 N}(\varepsilon)$ as parameters connected to $\left(\varepsilon_{1}^{2}, \varepsilon_{2}^{2}\right)$ via a diffeomorphism.

After some elements on pseudodifferential operators and an introduction of useful operators in section 8 , the section 9 , which plays a fundamental role in the paper, deals with the descent method. This consists in making a succession of change of variables in (1.11). First of all we show (Proposition 1) that there is a linear operator $\mathfrak{S}_{-1}: H_{o}^{s} \rightarrow H_{o}^{s}$ (odd functions) with a bounded inverse such that

$$
\mathfrak{S}_{-1}\left\{\nu \mathcal{D}^{2}+p \mathcal{D}+\mathfrak{G}_{1}+\mathfrak{G}^{*}\right\}=\mathfrak{L}+\mathfrak{A D}+\mathfrak{B}+\mathfrak{F}_{-1},
$$

where $\mathfrak{F}_{-1}$ is a pseudodifferential operator of order $-1, \mathfrak{A}$ and $\mathfrak{B}$ are zero order pseudodifferential operators, and the operator $\mathfrak{L}$ has the representation $\mathfrak{L}=-\nu \mathcal{D}^{2}+(-\Delta)^{1 / 2}, \quad$ where $e^{-i \mathbf{k} \cdot X} \Delta e^{i \mathbf{k} \cdot X}=-\mathbb{A} \tilde{\mathbf{k}} \cdot \tilde{\mathbf{k}}, \tilde{\mathbf{k}}=\left(k_{1}+\rho k_{2}, k_{2}\right)$

with a positive constant matrix $\mathbb{A}$. Moreover, the norms of operators $\mathfrak{A}, \mathfrak{B}$ and $\mathfrak{F}_{-1}$ do not exceed $c \varepsilon$. The main difficulty now is that the operator $\mathcal{D}$, in contrast to the case of symmetric waves, has an unbounded inverse. In order to cope with this difficulty we introduce the projection $\Pi$ such that the operator $\mathfrak{L}$ has a compact inverse $\mathfrak{L}^{-1}:(I-\Pi) H^{s}\left(\mathbb{R}^{2} /(2 \pi \mathbb{Z})^{2}\right) \rightarrow$ $(I-\Pi) H^{s}\left(\mathbb{R}^{2} /(2 \pi \mathbb{Z})^{2}\right)$. Next we apply the descent method developed in [16] to the operator $\Pi\left(\mathfrak{L}+\mathfrak{A D}+\mathfrak{B}+\mathfrak{F}_{-1}\right)$. The descent method along with estimates of commutators of $\Pi$ and zero order pseudodifferential operators leads to the representation (see Theorem 8)

$$
\mathfrak{S}\left\{\mathfrak{L}+\mathfrak{A D}+\mathfrak{B}+\mathfrak{F}_{-1}\right\} \mathfrak{T}=\Pi(\mathfrak{L}+\mathfrak{V}+\mathfrak{F}) \Pi+(\mathbf{I}-\Pi)(\mathfrak{L}+\mathfrak{P}),
$$

where the operators $\mathfrak{S}, \mathfrak{T}$ are bounded and have bounded inverses, $\mathfrak{V}$ is a bounded zero order pseudodifferential operator with small constant coefficients, $\mathfrak{P}$ is a small bounded operator, and $\mathfrak{F}$ is a pseudodifferential operator of order -1 . The operator in the right hand side is easily invertible once we 
control the invertibility of the main part, which is now with constant coefficients. There is a serious difference between the descent method presented here, and the one used in [16], since at some stage we need to split the Fourier plane in two parts, to be able to control the inverse of the operator $\mathcal{D}$ on the part of the Fourier plane where the full linear operator has an unbounded inverse. This means that the method used in this paper is more general that the one used in [16], and far more general than the one used in [15]. This implies a triangular final normal form for the operator and in section 9 we introduce the diophantine estimate (Theorem 11) allowing to control its inverse, estimated in section 10 (Theorems 12 and 13). Notice that this last estimate is valid all along the successive points of the Newton iteration scheme, and this provides a restriction on the choice of couples $(\rho, \nu)$, which corresponds to restrictions on the choice of amplitudes $\left(\varepsilon_{1}^{2}, \varepsilon_{2}^{2}\right)$ belonging to a set which has 0 as a Lebesgue point in $\mathbb{R}^{+2}$.

Section 12 applies extensively the result proved in [15] concerning the Nash-Moser Theorem with a parameter in a Cantor set. The existence of an asymmetrical travelling wave seen as a perturbation of a specific approximate solution, is the main result of the paper, as indicated in Theorem 2.

\subsubsection{Directional Stokes drift}

Knowing the rotation number of the velocity field $V$, which is the horizontal projection of the velocity of fluid particles, allows us to give the asymptotic direction of particles trajectories, and to compare them with the propagation direction of the waves. It is a remarkable fact that these directions are slightly different. We prove the following

Lemma 3 (Directional Stokes drift) In the frame moving with the velocity of the waves, the horizontal projection of the asymptotic direction taken by fluid particles makes an angle $\sim 4\left(\mu_{c}^{-2}\right)\left\{-\tau_{1} \varepsilon_{1}^{2}+\lambda^{4} \tau_{2} \varepsilon_{2}^{2}\right\}$ with the direction of propagation of the waves. There is a special value of the ratio $\varepsilon_{1}^{2} / \varepsilon_{2}^{2} \sim \lambda^{4} \tau_{2} / \tau_{1}$ for which both directions are identical.

\section{lineqorigin 2 Linearized equations at the origin and dispersion relation}

The linearization at the origin of the system (1.2), (1.3), (1.4) with $\mu=\mu_{c}$, $\mathbf{u}=\mathbf{u}_{0}$ leads to

$$
\mathcal{L}_{0} U:=\left(\begin{array}{c}
\mathcal{G}^{(0)} \psi-\mathbf{u}_{0} \cdot \nabla \eta \\
\mathbf{u}_{0} \cdot \nabla \psi+\mu_{c} \eta
\end{array}\right), \quad \text { where } \mathcal{G}^{(0)}=(-\Delta)^{1 / 2}
$$

linsyst1

is the Dirichlet-Neumann operator corresponding to a flat free surface. Now expanding in Fourier series, the system (2.1) gives for any $\mathbf{k} \in \Gamma^{\prime}$

$$
|\mathbf{k}| \widehat{\psi}_{\mathbf{k}}-i\left(\mathbf{k} \cdot \mathbf{u}_{0}\right) \widehat{\eta}_{\mathbf{k}}=i\left(\mathbf{k} \cdot \mathbf{u}_{0}\right) \widehat{\psi}_{\mathbf{k}}+\mu_{c} \widehat{\eta}_{\mathbf{k}}=0 .
$$

Hence, the dispersion relation reads

$$
\Delta\left(\mathbf{k}, \mu_{c}, \mathbf{u}_{0}\right) \stackrel{\text { def }}{=} \mu_{c}|\mathbf{k}|-\left(\mathbf{k} \cdot \mathbf{u}_{0}\right)^{2}=0 .
$$


Without restricting the generality, we choose vectors $K_{1}=\left(1, \tau_{1}\right)$ and $K_{2}=$ $\lambda\left(1,-\tau_{2}\right)$ and $\mathbf{u}_{0}=(1,0)$ (thanks to the invariance under rotations of the system), satisfying the dispersion relation (2.2). This implies

$$
\lambda=\frac{\cos \theta_{1}}{\cos \theta_{2}}, \mu_{c}=\cos \theta_{1}=\frac{1}{\left|K_{1}\right|}=\frac{\lambda^{2}}{\left|K_{2}\right|}
$$

where $\tau_{i}=\tan \theta_{i}$. Notice that we still have two free parameters $\tau_{1}, \tau_{2}$ in the problem. We assume in the following

Hypothesis $\mathbf{3}$ The only solutions $\mathbf{k} \in \Gamma^{\prime}$ of the dispersion relation (2.2) are $\mathbf{0}, \pm K_{1}, \pm K_{2}$.

Notice that we prove in particular at Theorem 10 that a stronger condition than hypothesis 3 is verified for almost all $\left(\tau_{1}, \tau_{2}\right)$ in the positive quadrant of the plane. For the linear operator $\mathcal{L}_{0}$ we have now the 4-dim kernel

$$
\zeta_{K_{1}}=\left(i, \frac{1}{\mu_{c}}\right) e^{i K_{1} \cdot X}, \zeta_{K_{2}}=\left(i, \frac{\lambda}{\mu_{c}}\right) e^{i K_{2} \cdot X}, \quad \zeta_{-K_{j}}=\bar{\zeta}_{K_{j}}, \quad j=1,2 .
$$

\section{Formal computation of asymmetrical 3-dimensional waves}

In this section we state the following

Theorem 4 Assume that $\left(\tau_{1}, \tau_{2}\right)$ satisfies the non resonance Hypothesis 3 for the dispersion equation (2.2). Then, for fixed $\left(\varepsilon_{1}, \varepsilon_{2}\right) \in \mathbb{R}^{2}$, and any $\mathbf{v} \in \mathbb{R}^{2}$, there is a formal expansion solution of the system (1.2), (1.3), (1.4), of the form

$$
\begin{aligned}
U & =(\psi, \eta)=\sum_{p+q+r+s \geq 1} A^{p} \bar{A}^{q} B^{r} \bar{B}^{s} U_{p q r s}, \\
\widetilde{\mu} & =\mu-\mu_{c}=\alpha_{1} \varepsilon_{1}^{2}+\alpha_{2} \varepsilon_{2}^{2}+O\left\{\left(\varepsilon_{1}^{2}+\varepsilon_{2}^{2}\right)^{2}\right\}, \\
\boldsymbol{\omega} & =\mathbf{u}-\mathbf{u}_{0}=\left(\omega_{1}, \omega_{2}\right), \omega_{1}=-\frac{\omega_{2}^{2}}{2}+O\left(\omega_{2}^{4}\right) \\
\omega_{2} & =\beta_{1} \varepsilon_{1}^{2}+\beta_{2} \varepsilon_{2}^{2}+O\left\{\left(\varepsilon_{1}^{2}+\varepsilon_{2}^{2}\right)^{2}\right\},
\end{aligned}
$$

with $A=\varepsilon_{1} e^{i K_{1} \cdot \mathbf{v}}, B=\varepsilon_{2} e^{i K_{2} \cdot \mathbf{v}}, \mathbf{v}$ corresponds to an arbitrary horizontal shift $\mathcal{T}_{\mathbf{v}}$, where $\left.U\right|_{\mathbf{v}=0}$ corresponds to $A=\varepsilon_{1}, B=\varepsilon_{2}$ both real,

$$
U_{1000}=\zeta_{K_{1}}, \quad U_{0010}=\zeta_{K_{2}}, \quad U_{p q r s}=\bar{U}_{q p s r},
$$

and $U_{2000}, U_{0020}, U_{1010}, U_{1001}$ given by $(A .6, A .7, A .8)$, and coefficients $\alpha_{j}, \beta_{j}$ given by (A.13), (A.14) depend analytically on $\left(\tau_{1}, \tau_{2}\right)$. Bifurcation parameters $\widetilde{\mu}$ and $\boldsymbol{\omega}$ are expanded in powers of $\varepsilon_{1}^{2}$ and $\varepsilon_{2}^{2}$. For $A=\varepsilon_{1}, B=\varepsilon_{2}, \psi$ is odd, while $\eta$ is even in $X$. Moreover, each formal solution $U\left(X, \varepsilon_{1}, \varepsilon_{2}\right)$ is invariant under the two symmetries $\mathcal{S}_{1}$ and $\mathcal{S}_{2}$ defined by

$$
\left(\mathcal{S}_{i} U\right)\left(X, \varepsilon_{1}, \varepsilon_{2}\right)=U\left(X+\boldsymbol{\lambda}_{i} / 2,(-1)^{i} \varepsilon_{1},(-1)^{i+1} \varepsilon_{2}\right) .
$$

Notice that for $\varepsilon_{1}=\varepsilon_{2}$ we have "diamond waves" only if $\left|K_{1}\right|=\left|K_{2}\right|$, i.e. if $\lambda=1$. For $\omega_{2}=0$, the direction of propagation is along the $x_{1}-$ axis. 
Proof The proof of Theorem 4 is given in Appendix A.

Remark 1 In the proof of Theorem 4, for the computation of the coefficients $U_{\text {pqrs }}$, we observe that at every successive increasing order in $|\varepsilon|$, we need to invert the linear operator $\mathcal{L}_{0}$ in a subspace orthogonal to ker $\mathcal{L}_{0}$. We have then at each step a division by $\Delta\left(\mathbf{k}, \mu_{c}, \mathbf{u}_{0}\right)$ with $\mathbf{k} \neq \mathbf{0}, \pm K_{1}, \pm K_{2}$. Since this quantity may be as close to 0 as we wish when $|\mathbf{k}|$ is large, we are faced with a small divisor problem, leading to a possibly diverging series for $U$, which is precisely the reason why we use below the Nash-Moser theorem. This problem does not occur in the case with surface tension, since it adds a term $|\mathbf{k}|^{3}$ in $\Delta$ which would imply a regularizing property of the inverse of $\mathcal{L}_{0}$ in $\left\{\operatorname{ker} \mathcal{L}_{0}\right\}^{\perp}$. In our present case this operator is unbounded, and its properties are made precise in section 10.

Remark 2 The set of formal solutions is defined up to an arbitrary horizontal translation, which makes a torus of formal solutions for fixed $\left(\tau_{1}, \tau_{2}, \varepsilon_{1}, \varepsilon_{2}\right)$. In fact we replace the initial bifurcation parameters $\mu$ and $\mathbf{u}$ by $\varepsilon_{j}=\left|\left(U, \zeta_{K_{j}}\right)\right|$, $j=1,2$, which is eligible as soon as $\alpha_{1} \beta_{2} \neq \alpha_{2} \beta_{1}$, which corresponds to the condition

$$
a_{1} b_{2}-a_{2} b_{1} \neq 0
$$

where $a_{j}, b_{j}$ are given in Appendix A. The left hand side of this condition is a non trivial analytic function of $\tau_{1}$ and $\tau_{2}$ (as it can be checked easily with the formulas giving $a_{j}, b_{j}, j=1,2$ in Appendix A), hence this condition holds for all $\left(\tau_{1}, \tau_{2}\right)$ except maybe on a curve of the $\left(\tau_{1}, \tau_{2}\right)$ plane.

Remark 3 In [2] Craig and Nicholls were the first to obtain a formal asymptotic expansion of asymmetrical waves. However their result needs to be corrected and simplified, because they do not use properly the symmetries of the system, by fixing a priori real amplitudes for $A$ and $B$ before using the translational invariance.

\section{Linearized system in $(\psi, \eta) \neq 0$}

To avoid ambiguity in the proof of existence of a solution, because of the torus family of formal solutions, we need to specify what solution we are looking for. Indeed, from now on we restrict the study to solutions invariant under the symmetry $\mathcal{S}_{0}$, and such that $A=\varepsilon_{1}, B=\varepsilon_{2}$ are real positive.

Let us consider the nonlinear system (1.2). We define the horizontal projection $V$ of the velocity of fluid particles by

$$
V=\mathbf{u}+\nabla \psi-\mathfrak{b} \nabla \eta, \quad \mathfrak{b}=\frac{1}{1+|\nabla \eta|^{2}}\{\nabla \eta \cdot(\mathbf{u}+\nabla \psi)\},
$$

and it is shown in [16] that the differential of $\mathcal{F}$ with respect to $U$ takes the form

$$
\partial_{U} \mathcal{F}(U, \mu, \mathbf{u})[\delta U]=\mathcal{L}_{\mu, \mathbf{u}}(U)[\delta \phi, \delta \eta]+\mathcal{R}(\mathcal{F}, U)[\delta U],
$$

where $\delta U=(\delta \psi, \delta \eta) \in \mathbb{H}^{m}\left(\mathbb{R}^{2} / \Gamma\right)$,

$$
\delta \phi=\delta \psi-\mathfrak{b} \delta \eta,
$$


and where the linear symmetric operator $\mathcal{L}_{\mu, \mathbf{u}}(U)$ is defined by

$$
\begin{gathered}
\mathcal{L}_{\mu, \mathbf{u}}(U)=\left(\begin{array}{cc}
\mathcal{G}_{\eta} & \mathcal{J}^{*} \\
\mathcal{J} & \mathfrak{a}
\end{array}\right), \\
\mathcal{J}=V \cdot \nabla(\cdot), \quad \mathcal{J}^{*}=-\nabla \cdot(V(\cdot)) \quad \mathfrak{a}=V \cdot \nabla \mathfrak{b}+\mu .
\end{gathered}
$$

The rest $\mathcal{R}$ has the form

$$
\begin{gathered}
\mathcal{R}(\mathcal{F}, U)[\delta U]=\left(\mathcal{R}_{1}(\mathcal{F}, U)[\delta U], 0\right) \\
\mathcal{R}_{1}(\mathcal{F}, U)[\delta U]=-\mathcal{G}_{\eta}\left(\frac{\mathcal{F}_{1} \delta \eta}{1+(\nabla \eta)^{2}}\right)+\nabla \cdot\left(\frac{\mathcal{F}_{1} \delta \eta}{1+(\nabla \eta)^{2}} \nabla \eta\right)
\end{gathered}
$$

and cancels when $U$ is a solution of $\mathcal{F}(U, \mu, \mathbf{u})=0$. For $U \in \mathbb{H}_{(S)}^{m}$

$$
V \in\left(H_{e}^{m-1}\left(\mathbb{R}^{2} / \Gamma\right)\right)^{2}, \mathfrak{b} \in H_{o}^{m-1}\left(\mathbb{R}^{2} / \Gamma\right), \mathfrak{a} \in H_{e}^{m-2}\left(\mathbb{R}^{2} / \Gamma\right),
$$

where the indexes $e$ or $o$ means "even" or "odd" with respect to $X$. Moreover we have the following "tame" estimates proved in [16]:

tame coef Lemma 4 Let $U \in \mathbb{H}_{(S)}^{m}, m \geq 3$. Then, there exists $M_{3}>0$ such that for $\|U\|_{3} \leq M_{3}$, one has

$$
\begin{gathered}
\|V-\mathbf{u}\|_{m-1}+\|\mathfrak{a}-\mu\|_{m-2} \leq c_{m}\left(M_{3}\right)\|U\|_{m} \\
\left.\|\mathcal{R}(\mathcal{F}, U)[\delta U]\|\right|_{m-2} \leq c_{s}\left(M_{3}\right)\left\{\left\|\mathcal{F}_{1}\right\|_{2}\left(\|\eta\|_{m}\|\delta \eta\|_{2}+\|\delta \eta\|_{m-1}\right)+\left\|\mathcal{F}_{1}\right\|_{m-1}\|\delta \eta\|_{2}\right\} .
\end{gathered}
$$

The main difficulty in using the Nash Moser theorem, is to invert the approximate linearized system, i.e. invert the linear system

$$
\mathcal{L}_{\mu, \mathbf{u}}(U)[\delta \phi, \delta \eta]=(f, g),
$$

which leads to the scalar equation

$$
-\mathcal{J}^{*}\left(\frac{1}{\mathfrak{a}} \mathcal{J}(\delta \phi)\right)+\mathcal{G}_{\eta}(\delta \phi)=h \text { with } h=f-\mathcal{J}^{*}\left(\frac{1}{\mathfrak{a}} g\right) \in H_{o}^{s}\left(\mathbb{R}^{2} / \Gamma\right)
$$

and where we look for $\delta \phi$ in some $H_{o}^{s-r}\left(\mathbb{R}^{2} / \Gamma\right)$. In the Newton iteration method, we linearize around some $U(\cdot, \varepsilon) \in \mathbb{H}_{(S)}^{m}$, where $\varepsilon=\left(\varepsilon_{1}, \varepsilon_{2}\right)$, which is not only invariant under $\mathcal{S}_{0}$, but also invariant under the symmetries $\mathcal{S}_{1}$ and $\mathcal{S}_{2}$. It results that at each step of the iteration, $\mathcal{S}_{j} h=h$, and coefficients $V, \mathfrak{a}, \mathfrak{b}$ are invariant under $\mathcal{S}_{j}$, and we expect to find $\delta \phi$ invariant under $\mathcal{S}_{j}$, $j=1,2$.

In the next section we find a formal diffeomorphism of the torus which allows to transform the coefficients of leading derivatives in (4.6) into constants. For avoiding to use infinitely many times the Nash-Moser implicit function theorem in this paper, in an intricate way, this diffeomorphism will constitute a part of the unknowns in the Nash-Moser theory which is used here. 


\section{Formal study of the change of variable}

Rotation number. Let us derive the equation for the diffeomorphism of the torus which brings equation (4.6) to a canonical form with constant coefficients in the leading part. The idea below is to find a diffeomorphism of the form

$$
X(Y)=\mathbb{T}^{-1} Y+\mathcal{W}(Y, \boldsymbol{\varepsilon}) \text { with } \mathbb{T}^{-1} Y=\frac{1}{2 \pi}\left(y_{1} \boldsymbol{\lambda}_{1}, y_{2} \boldsymbol{\lambda}_{2}\right) .
$$

The function $\mathcal{W}$ being $(2 \pi \mathbb{Z})^{2}$ - periodic, odd in $Y$, and invariant under the symmetries

$$
\left(\widetilde{\mathcal{S}}_{i} U\right)\left(Y, \varepsilon_{1}, \varepsilon_{2}\right)=\left(Y+\pi \mathbf{e}_{i},(-1)^{i} \varepsilon_{1},(-1)^{i+1} \varepsilon_{2}\right), \quad i=1,2 .
$$

This diffeomorphism should be chosen in such a way that it transforms the coefficients of the leading derivatives in (4.6) into constants. Since the second order derivative contains twice a derivative along the direction $V$, let us look for diffeomorphism (5.1) satisfying

$$
\varrho=\frac{1}{\sqrt{\nu} f(X(Y))} X^{\prime}(Y)^{-1} V(X(Y)) \text { where } \varrho=(1, \rho),
$$

and $f(X)$ is computed below for satisfying our purpose. It should be noticed that the mapping $X \rightarrow Y(X)$ takes the torus $\mathbb{R}^{2} / \Gamma$ into the torus $(\mathbb{R} / 2 \pi \mathbb{Z})^{2}$, and the vector field $V$ into a vector field with straight lines trajectories

$$
\dot{Y}=\sqrt{\nu} f(X(Y)) \varrho
$$

Depending on the context, we use the following notations for the first order differential operator $\mathcal{D}$ defined by

$$
\mathcal{D}:=\partial_{y_{1}}+\rho \partial_{y_{2}} \equiv \varrho \cdot \nabla \text { with } \mathcal{D} X \equiv \boldsymbol{\varrho} \cdot \nabla X \equiv X^{\prime} \varrho
$$

so that (5.2) reads

$$
\mathcal{D} X=\nu^{-1 / 2} f(X(Y))^{-1} V(X(Y)) .
$$

Let us now consider the second order derivative term $-\mathcal{J}^{*}\left(\frac{1}{\mathfrak{a}} \mathcal{J}(\delta \phi)\right)$ of the linear equation (4.6), we see that it reads as

$$
\nabla_{X} \cdot\left(\frac{V}{\mathfrak{a}}\left(V \cdot \nabla_{X}(\delta \phi)\right)\right)=\frac{V}{\mathfrak{a}} \cdot \nabla_{X}\left(V \cdot \nabla_{X}(\delta \phi)\right)+\left(V \cdot \nabla_{X}(\delta \phi)\right) \nabla_{X} \cdot\left(\frac{V}{\mathfrak{a}}\right)
$$

and using the new coordinates $Y$, denoting from now on with a tilde the functions of $X$ expressed as functions of $Y$ with $X(Y)$, we obtain with $\delta \phi(X(Y))=u(Y)$

$$
\left.\nabla_{X} \cdot\left(\frac{V}{\mathfrak{a}}\left(V_{X} \cdot \nabla(\delta \phi)\right)\right)=\nu\left(\widetilde{\mathfrak{a}^{-1} f^{2}}\right) \mathcal{D}^{2} u+\sqrt{\nu}\left[\nabla_{X} \widetilde{\cdot\left(\mathfrak{a}^{-1}\right.} V f\right)\right] \mathcal{D} u
$$


Leading first order derivative. Define the matrices $\mathbb{Q}$ and $\mathbb{G}=\left(g_{i j}\right)_{2 \times 2}$ of the first fundamental form of the free surface, respectively in the parametric representations $\{x=(X, \eta(X)\}$ and $\{x=(X(Y), \widetilde{\eta}(Y)\}$ by

$$
\mathbb{Q}(X)=\mathbf{I}+\nabla_{X} \eta(X) \otimes \nabla_{X} \eta(X), \quad \mathbb{G}(Y)==X^{\prime *}(Y) \widetilde{\mathbb{Q}}(Y) X^{\prime}(Y),
$$

and set $J(Y)=-\operatorname{det} X^{\prime}(Y)>0$ because $\operatorname{det} \mathbb{T}<0$. It is shown in [16], see also Theorem 7 in Section 8, that the leading order of the Dirichlet-Neumann operator $\mathcal{G}_{\eta}$, in variable $Y$, is a first order pseudodifferential operator with the symbol $G_{1}$ given by (8.11). We obtain from (8.11)

$$
\begin{aligned}
G_{1}(Y, \mathbf{k})= & \frac{1}{J}\left\{g_{22}\left(k_{1}+\rho k_{2}\right)^{2}-2\left(g_{12}+\rho g_{22}\right)\left(k_{1}+\rho k_{2}\right) k_{2}+\right. \\
& \left.+\left(g_{11}+2 \rho g_{12}+\rho^{2} g_{22}\right) k_{2}^{2}\right\}^{1 / 2} .
\end{aligned}
$$

symbolG_1new

In this expression we specify the symbol $i\left(k_{1}+\rho k_{2}\right)$ of the differential operator $\mathcal{D}$ which appears already with degree 2 in the leading order derivative in (5.4). So it is clear (because of the half power) that the next important coefficient of the differential operator occuring in (4.6) is the coefficient of $k_{2}^{2}$ in the square root. This coefficient takes the following form:

$$
\frac{1}{J}\left(g_{11}+2 \rho g_{12}+\rho^{2} g_{22}\right)^{1 / 2} \equiv \frac{1}{J}\left(X^{\prime *} \mathbb{Q} X^{\prime} \varrho \cdot \varrho\right)^{1 / 2}=\frac{1}{J}(\mathbb{Q} \mathcal{D} X \cdot \mathcal{D} X)^{1 / 2} .
$$

We choose now the function $f$ such that

$$
\left(\widetilde{\mathfrak{a}^{-1} f^{2}}\right)=J^{-1}(\mathbb{Q} \mathcal{D} X \cdot \mathcal{D} X)^{1 / 2}
$$

It results that the expression of the symbol of the leading order terms of the differential operator in (4.6), takes the form

$$
\chi\left\{-\nu\left(k_{1}+\rho k_{2}\right)^{2}+\left[G_{1,11}\left(k_{1}+\rho k_{2}\right)^{2}+2 G_{1,12}\left(k_{1}+\rho k_{2}\right) k_{2}+k_{2}^{2}\right]^{1 / 2}\right\}
$$

with $\chi(Y)=\left(\widetilde{\left(\mathfrak{a}^{-1} f^{2}\right.}\right)(Y)$. Now replacing $f$ by its value, we obtain from $(5.3)$

$$
\mathcal{D} X=J^{1 / 2}(\nu \widetilde{\mathfrak{a}})^{-1 / 2}(\widetilde{\mathbb{Q}} \mathcal{D} X \cdot \mathcal{D} X)^{-1 / 4} \widetilde{V} .
$$

Making the scalar product of both sides by $\widetilde{\mathbb{Q}} \mathcal{D} X$, we can eliminate the term $\widetilde{\mathbb{Q}} \mathcal{D} X \cdot \mathcal{D} X$ and rewrite this equation in the equivalent form

$$
\left.\mathcal{D} X=\left|X^{\prime}\right|^{1 / 3} \mathbf{F}(U, \nu, \mu, \mathbf{u})\right),
$$

$\mathrm{F}(\mathrm{U}, \mathrm{mu}) 3$

where

$$
\mathbf{F}=-(\nu \widetilde{\mathfrak{a}})^{-1 / 3}\left(\widetilde{V}^{2}+\left(\widetilde{V} \cdot \widetilde{\nabla_{X} \eta}\right)^{2}\right)^{-1 / 6} \widetilde{V}
$$

$V$ is defined by (4.1), and $\mathfrak{a}$ by (4.5). Here we observe that the absolute value $J$ of the determinant of $X^{\prime}(Y)$ is at the power $1 / 3$. Hence, with a change of variables of the form (5.1), which satisfies (5.10), we succeed in putting constant coefficients in front of the leading derivatives in (4.6). It remains to find $\mathcal{W}(Y)$ in $X(Y)$ (see $(5.1)$ ), and the scalars $\rho$ and $\nu$. 


\section{Extended system. Problem formulation and approximate solutions}

Extended system Note that the canonical diffeomorphism $Y=Y(X)$ takes the integral curves of the vector field $V$ onto lines $y_{2}=\rho y_{1}+$ const., which means that these curves form a foliation of $\mathbb{R}^{2} / \Gamma$ with the rotation number $\rho$. Hence the topological restriction will be satisfied if we add equation (5.10) to the basic operator equation (1.2). Thus we arrive at the following extended problem

Problem 1 . For given positive $\tau_{1}, \tau_{2}$, find periodic functions $\mathbb{R}^{2} / \Gamma \ni X \mapsto$ $U(X)=(\psi(X), \eta(X))$, a diffeomorphism $\mathbb{R}^{2} /(2 \pi \mathbb{Z})^{2} \rightarrow \mathbb{R}^{2} / \Gamma: Y \mapsto X(Y)=$ $\mathbb{T}^{-1} Y+\mathcal{W}(Y)$, parameters $\mu, \nu, \rho$, and a constant unit vector $\mathbf{u}$ satisfying the system

$$
\begin{aligned}
\mathcal{F}_{1}(U, \mathbf{u}) & =0, \quad \mathcal{F}_{2}(U, \mu, \mathbf{u})=0, \\
\mathcal{F}_{3}(U, X, \rho, \nu, \mu, \mathbf{u}) & \left.=: \mathcal{D} X-\left|X^{\prime}\right|^{1 / 3} \mathbf{F}(U, \nu, \mu, \mathbf{u})\right)=0, \\
\psi\left(X+m \boldsymbol{\lambda}_{1}+n \boldsymbol{\lambda}_{2}\right) & =\psi(X), \quad \eta\left(X+m \boldsymbol{\lambda}_{1}+n \boldsymbol{\lambda}_{2}\right)=\eta(X), \\
\mathcal{W}\left(Y+2 \pi m \mathbf{e}_{1}+2 \pi n \mathbf{e}_{2}\right) & =\mathcal{W}(Y), \quad X(Y)=\mathbb{T}^{-1} Y+\mathcal{W}(Y) .
\end{aligned}
$$

Here $\mathcal{F}_{1}$ and $\mathcal{F}_{2}$ are defined by (1.3), (1.4), and $\mathbf{F}$ is defined by (5.11).

We are looking for a solution of the extended Problem 1, i.e., for functions $U=(\psi, \eta)$, a diffeomorphism $X=X(Y)$, a constant vector $\mathbf{u}$ and a parameter $\mu$. The governing equations in Problem 1 depend also on parameters $\rho$, $\nu$ and on the lattice of periods. The lattice is completely determined by the positive parameters $\tau_{1}$ and $\tau_{2}$, thanks to (2.3).

Approximate solutions. The extended problem also has a sequence of approximate solutions in the form of polynomials in the small parameter $\varepsilon$. In particular, we state the following

Lemma 5 Under the conditions of Theorem 4 and assuming that $\lambda \notin \mathbb{Q}$, there is a function $\mathcal{W}$, and scalars $\rho$ and $\nu>0$, formal solutions of the equation (5.10), under the form of formal powers series in $\varepsilon_{1}$ and $\varepsilon_{2}$, such that $\mathcal{W}$ is $(2 \pi \mathbb{Z})^{2}$ - periodic, odd in $Y$, and invariant under the symmetries $\widetilde{\mathcal{S}}_{j}, j=1,2$, and the scalar power series for $\rho$ and $\nu$ take the form

$$
\begin{gathered}
\rho=\rho\left(\varepsilon_{1}^{2}, \varepsilon_{2}^{2}\right)=\lambda+\rho_{1} \varepsilon_{1}^{2}+\rho_{2} \varepsilon_{2}^{2}+O\left\{\left(\varepsilon_{1}^{2}+\varepsilon_{2}^{2}\right)^{2}\right\} \\
\nu=\nu\left(\varepsilon_{1}^{2}, \varepsilon_{2}^{2}\right)=\nu_{c}+\nu_{1} \varepsilon_{1}^{2}+\nu_{2} \varepsilon_{2}^{2}+O\left\{\left(\varepsilon_{1}^{2}+\varepsilon_{2}^{2}\right)^{2}\right\},
\end{gathered}
$$

where $\rho_{1}, \rho_{2}, \nu_{1}, \nu_{2}$ are given by (B.9), (B.10), (B.13), (B.14), and

$$
\nu_{c}=\left\{\mu_{c} \lambda\left(\tau_{1}+\tau_{2}\right)\right\}^{-1} .
$$

Proof The proof of Lemma 5 is in Appendix B. 
Remark 4 It should be noticed that if $\lambda=r / s \in \mathbb{Q}$, there is an obstruction in the computation of $\mathcal{W}$ at order $|\varepsilon|^{r+s}$, as seen in Appendix B. This means that if we only need an approximate solution at order $|\varepsilon|^{8}$, it would be sufficient to assume $\lambda \neq r / s$ for $r+s \leq 8$. However, in the following we need that $\lambda \notin \mathbb{Q}$ to allow the diophantine condition (9.17) to hold uniformly as $\rho$ approaches $\lambda$.

By virtue of Theorem 4 and Lemma 5 for any fixed lattice of periods such that $\lambda \notin \mathbb{Q}$, and $m>3$, the Problem 1 has an approximate solution

$$
\begin{aligned}
& U_{m}(\varepsilon)=\left(\psi_{m}(\varepsilon), \eta_{m}(\varepsilon)\right), \quad X_{m}(\varepsilon)=\mathbb{T}^{-1} Y+\mathcal{W}_{m}(\varepsilon), \\
& \mathbf{u}_{m}(\varepsilon)=\mathbf{u}_{0}+\boldsymbol{\omega}_{m}(\varepsilon), \quad \mu_{m}(\varepsilon), \quad \rho_{m}(\varepsilon), \quad \nu_{m}(\varepsilon),
\end{aligned}
$$

which satisfies the equations of Problem 1 with an accuracy up to order $|\varepsilon|^{m+1}$, and depends on the parameter $\varepsilon=\left(\varepsilon_{1}, \varepsilon_{2}\right)$. More precisely, the approximate solution is a polynomial of degree $m$ in $\varepsilon$.

We are looking for an explicit solution of the Problem 1 in the form

$$
\begin{aligned}
& U=U_{4 m}(\varepsilon)+|\varepsilon|^{2 m} \breve{U}, \quad X=X_{4 m}(\varepsilon)+|\varepsilon|^{2 m} \breve{\mathcal{W}}, \\
& \mathbf{u}(\varepsilon)=\mathbf{u}_{4 m}(\varepsilon)+|\varepsilon|^{2 m} \breve{\boldsymbol{\omega}}, \quad \mu=\mu_{4 m}(\varepsilon)+|\varepsilon|^{2 m} \breve{\mu}, \\
& \rho=\rho_{4 m}(\varepsilon), \quad \nu=\nu_{4 m}(\boldsymbol{\varepsilon})
\end{aligned}
$$

with new unknowns $\breve{U}, \breve{\mathcal{W}}, \breve{\boldsymbol{\omega}}, \breve{\mu} \sim o\left(|\varepsilon|^{m}\right)$. In this framework, $\rho_{4 m}(\varepsilon)$ and $\nu_{4 m}(\varepsilon)$ are considered as given functions completely determined by Lemma 5 where the series is truncated at order $4 \mathrm{~m}$. Notice that this modifies the former definition of $\varepsilon=\left(\varepsilon_{1}, \varepsilon_{2}\right)$ (see Remark 2). Therefore, the natural bifurcation parameter is $(\mu, \mathbf{u}) \in \mathbb{R}^{+} \times S^{1}$ near $\left(\mu_{c}, \mathbf{u}_{0}\right)$. We expressed the formal family of bifurcating solutions in terms of $\varepsilon=\left(\varepsilon_{1}, \varepsilon_{2}\right)$, by choosing $\varepsilon$ instead of $(\mu, \mathbf{u})$ which is valid as soon as the following condition holds (see Theorem 4) $\alpha_{1} \beta_{2}-\alpha_{2} \beta_{1} \neq 0$ which is equivalent to condition (3.1). In the proof of the existence theorem, we prefer to use the parameter $(\rho, \nu) \in \mathbb{R}_{+}^{2}$ in the neighborhood of $\left(\lambda, \nu_{c}\right)$. This choice is eligible when the condition

$$
\rho_{1} \nu_{2}-\rho_{2} \nu_{1} \neq 0
$$

holds, where $\rho_{j}$ and $\nu_{j}$ are given by (B.9), (B.10), (B.13), (B.14). The conditions (3.1) and (6.4) are expressed as non zero values of two non trivial analytic functions of $\left(\tau_{1}, \tau_{2}\right)$. As it is easy to check for $\tau_{1} \sim \tau_{2}$, these conditions are non trivial by using the asymptotic values of $\rho_{j}$ and $\nu_{j}$ given in Appendix B. It results that for all values of $\left(\tau_{1}, \tau_{2}\right)$ except maybe on two curves of the plane, conditions (3.1) and (6.4) are satisfied. Choosing $(\rho, \nu)$ as new parameters, implies now that $U, X, \mu$ and $\mathbf{u}$ are unknown functions of $\rho, \nu$.

Moreover, with the assumption (6.4), the order $|\varepsilon|^{2}$ is equivalent to order $O\left(|\rho-\lambda|+\left|\nu-\nu_{c}\right|\right)$, and we have the following

for (rho,nu) Lemma 6 Assume that Condition (6.4) holds, then there exists $\epsilon_{0}>0$ such that for $0<\epsilon<\epsilon_{0}$, there is an analytic diffeomorphism $\left(\varepsilon_{1}^{2}, \varepsilon_{2}^{2}\right) \mapsto$ $(\rho, \nu)$ from the triangular region of $\mathbb{R}^{+2}: 0<\varepsilon_{1}^{2}+\varepsilon_{2}^{2}<\epsilon$ onto an open 

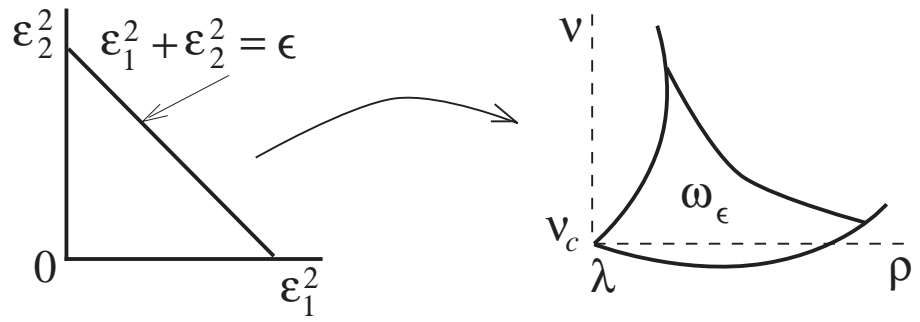

Fig. 1 Diffeomorphism of Lemma 6 and domain $\omega_{\epsilon}$

curved triangular domain $\omega_{\epsilon}$ of $\mathbb{R}^{2}$ (see Figure 1) bounded by the following two smooth curves originated in $\left(\lambda, \nu_{c}\right):\left\{\left(\rho_{4 m}\left(0, \varepsilon_{2}\right), \nu_{4 m}\left(0, \varepsilon_{2}\right)\right) ; 0<\varepsilon_{2}<\sqrt{\epsilon}\right\}$, $\left\{\left(\rho_{4 m}\left(\varepsilon_{1}, 0\right), \nu_{4 m}\left(\varepsilon_{1}, 0\right)\right) ; 0<\varepsilon_{1}<\sqrt{\epsilon}\right\}$, and the smooth curve

$$
\left.\left\{\left(\rho_{4 m}(\sqrt{\epsilon} \cos \theta, \sqrt{\epsilon} \sin \theta), \nu(\sqrt{\epsilon} \cos \theta, \sqrt{\epsilon} \sin \theta)\right)\right) ; 0<\theta<\pi / 2\right\} .
$$

More precisely, we can invert the diffeomorphism (by using the implicit function theorem for $\epsilon_{0}$ small enough, which then leads to

$$
\varepsilon_{i}^{2}=(-1)^{j} \frac{\nu_{j}(\rho-\lambda)-\rho_{j}\left(\nu-\nu_{c}\right)}{\rho_{1} \nu_{2}-\rho_{2} \nu_{1}}+O\left\{\left(|\rho-\lambda|+\left|\nu-\nu_{c}\right|\right)^{2}\right\}
$$

where $i, j=1,2, i \neq j$. The domain $\omega_{\epsilon}$ is then obtained in expressing $\varepsilon_{1}^{2}>0$, $\varepsilon_{2}^{2}>0, \varepsilon_{1}^{2}+\varepsilon_{2}^{2}<\epsilon$, and the boundaries of $\omega_{\epsilon}$ are at order $O\left\{\left(|\rho-\lambda|+\left|\nu-\nu_{c}\right|\right)^{2}\right\}$ close to the lines

$$
\begin{aligned}
\nu_{i}(\rho-\lambda)-\rho_{i}\left(\nu-\nu_{c}\right) & =0, i=1,2 \\
\left(\nu_{2}-\nu_{1}\right)(\rho-\lambda)+\left(\rho_{1}-\rho_{2}\right)\left(\nu-\nu_{c}\right) & =\epsilon\left(\rho_{1} \nu_{2}-\rho_{2} \nu_{1}\right) .
\end{aligned}
$$

pend (rho,nu) Remark 5 Notice that $\mu_{4 m}(\varepsilon), \mathbf{u}_{4 m}(\varepsilon),|\varepsilon|^{2 m}=\left(\varepsilon_{1}^{2}+\varepsilon_{2}^{2}\right)^{m}$ are analytic functions of $(\rho, \nu) \in \omega_{\epsilon_{0}}$.

\section{Linearization}

This section is devoted to the computation of the differential of the mapping $(U, X, \rho, \nu, \mu, \mathbf{u}) \mapsto\left(\mathcal{F}_{1}, \mathcal{F}_{2}, \mathcal{F}_{3}\right)$ and to state the strategy for inverting it in a neighborhood of $\left(0, \mathbb{T}^{-1}, \lambda, \nu_{c}, \mu_{c}, \mathbf{u}_{0}\right)$, for fixed $(\rho, \nu)$.

Conjugacy problem. In this paragraph we show that the nonlinear differential operator $\mathcal{F}_{3}$ is related to the class of "conjugacy problems". Assume that $\mathbf{F}: \mathbb{R}^{d} \rightarrow \mathbb{R}^{d}$ is a smooth mapping, $\varrho \in \mathbb{R}^{d}$ is a non-zero vector, $\alpha$ is an arbitrary real number. For any vector field $X: \mathcal{O} \rightarrow \mathbb{R}^{d}$ defined on an open set $\mathcal{O} \subset \mathbb{R}^{d}$, we set

$$
\mathcal{H}(X)=: \varrho \cdot \nabla X-\left|X^{\prime}\right|^{\alpha} \mathbf{F} \circ X
$$


where $X^{\prime}(Y)$ is the Jacobian matrix of the mapping $Y \mapsto X(Y)$ and $\left|X^{\prime}(Y)\right|=$ $\operatorname{det} X^{\prime}(Y)$. This relation determines the differential operator $\mathcal{H}$ which is strongly nonlinear when $\alpha \neq 0$ and weakly nonlinear when $\alpha=0$. We claim that for any $\alpha$, the linearization of the equation $\mathcal{H}=0$ can be reduced, by a linear change of variables, to a standard canonical form with constant coefficients, which does not depend on $\mathbf{F}$. This results from the following lemma.

conjugate1 Lemma 7 Let $X \in C^{2}(\mathcal{O})^{d},\left|X^{\prime}(Y)\right| \neq 0$ in $\mathcal{O}$, and the notation

$$
D_{X} \mathcal{H}(X)[\delta X]=\lim _{t \rightarrow 0} \frac{1}{t}(\mathcal{H}(X+t \delta X)-\mathcal{H}(X))
$$

stands for the Gateaux derivative of $\mathcal{H}$. Then, for any $v \in C^{1}(\mathcal{O})^{d}$,

$$
D_{X} \mathcal{H}(X)\left[X^{\prime} v\right]=X^{\prime}(\varrho \nabla v-\alpha(\operatorname{div} v) \varrho)+v \cdot \nabla \mathcal{H}(X)+\alpha(\operatorname{div} v) \mathcal{H}(X)
$$

conjugate3

Proof We have for $\delta X=X^{\prime} v$,

$$
\varrho \nabla \delta X=X^{\prime} \varrho \nabla v+\sum_{i} v_{i} \varrho \nabla \partial_{y_{i}} X \text { and } D_{X} \mathbf{F}(X)[\delta X]=\sum_{i} v_{i} \mathbf{F}^{\prime}(X) \partial_{y_{i}} X
$$

Note that

$$
D_{X}\left|X^{\prime}(Y)\right|[\delta X]=\left|X^{\prime}(Y)\right| \operatorname{div} v+\sum_{i} v_{i} \partial_{y_{i}}\left|X^{\prime}(Y)\right|
$$

Combining this result with (7.3) and using the identity

$$
D_{X}\left(\left|X^{\prime}\right|^{\alpha} \mathbf{F}(X)\right)[\delta X]=\alpha\left|X^{\prime}(Y)\right|^{\alpha-1}\left\{D_{X}\left(\left|X^{\prime}\right|\right)[\delta X]\right\} \mathbf{F}+\left|X^{\prime}\right|^{\alpha} D_{X} \mathbf{F}[\delta X]
$$

we arrive at

$$
\begin{aligned}
D_{X} \mathcal{H}(X)\left[X^{\prime} v\right] & =X^{\prime}(\varrho \cdot \nabla v)-\alpha \operatorname{div} v\left|X^{\prime}\right|^{\alpha} \mathbf{F}+ \\
& +\sum_{i} v_{i}\left(\varrho \cdot \nabla \partial_{y_{i}} X-\left|X^{\prime}\right|^{\alpha} F^{\prime} \partial_{y_{i}} X-\alpha\left|X^{\prime}\right|^{\alpha-1} \partial_{y_{i}}\left|X^{\prime}\right| \mathbf{F}\right) \\
& =X^{\prime}(\varrho \cdot \nabla v)+v \nabla \mathcal{H}(X)-\alpha \operatorname{div} v\left|X^{\prime}\right|^{\alpha} \mathbf{F} .
\end{aligned}
$$

On the other hand, we have

$$
\mid X^{\prime}{ }^{\alpha} \mathbf{F} \circ X=\varrho \cdot \nabla X-\mathcal{H}(X)=X^{\prime} \varrho-\mathcal{H}(X)
$$

Combining the results above leads to (7.2) and the lemma follows. 
Linearization of the operator $\widetilde{\mathcal{F}}$. In this paragraph we give the canonical representation for the Gateaux derivative of the nonlinear operator $\widetilde{\mathcal{F}}=$ $\left(\mathcal{F}_{1}, \mathcal{F}_{2}, \mathcal{F}_{3}\right)$ defined by $(1.3),(1.4),(6.1)$. We shall consider $\widetilde{\mathcal{F}}$ as an operator over the space of $\Gamma$-periodic functions $(\psi, \eta)$, diffeomorphic mappings $X$ : $\mathbb{R}^{2} /(2 \pi \mathbb{Z})^{2} \rightarrow \mathbb{R}^{2} / \Gamma$, parameters $\mu$, and unit vectors $\mathbf{u}$. The following theorem is the main result of this subsection, proved in Appendix D. Further we denote systematically with a tilde the functions of $X$ expressed as functions of $Y$ with $X(Y)$.

Theorem 5 Let functions $U=(\psi(X), \eta(X))$ and $\delta U=(\delta \psi(X), \delta \eta(X))$ be of class $\mathcal{C}^{3}\left(\mathbb{R}^{2} / \Gamma\right)$, a mapping $Y \mapsto X(Y)=\mathbb{T}^{-1} Y+\mathcal{W}(Y), \mathcal{W} \in$ $\left\{\mathcal{C}^{3}\left(\mathbb{R}^{2} /(2 \pi \mathbb{Z})^{2}\right)\right\}^{2}$, be a diffeomorphism of $\mathbb{R}^{2}$, and a mapping $v$ be of class $\left\{\mathcal{C}^{3}\left(\mathbb{R}^{2} /(2 \pi \mathbb{Z})^{2}\right)\right\}^{2}$. Let also $\delta \mathbf{u}$ be a constant vector, and $\delta \mu$ be a positive number. Furthermore, assume that functions $\delta \phi \in \mathcal{C}^{2}\left(\mathbb{R}^{2} / \Gamma\right), u, \varsigma \in$

$\mathcal{C}^{2}\left(\mathbb{R}^{2} /(2 \pi \mathbb{Z})^{2}\right)$ and the mapping $\delta X \in\left\{\mathcal{C}^{3}\left(\mathbb{R}^{2} /(2 \pi \mathbb{Z})^{2}\right)\right\}^{2}$ are related to $\delta U$ and $v$ by

$$
\begin{gathered}
\delta \phi=\delta \psi-\mathfrak{b} \delta \eta, \quad \delta X(Y)=X^{\prime}(Y) v(Y), \\
u(Y)=\widetilde{\delta \phi}(Y), \quad \varsigma(Y)=\widetilde{\delta \eta}(Y) .
\end{gathered}
$$

Then

$$
\begin{gathered}
\left(D_{(U, \mathbf{u})} \mathcal{F}_{1}(U, \mathbf{u})[\delta U, \delta \mathbf{u}]\right) \circ X(Y)=K \overline{\mathcal{G}} u-\frac{1}{H} \mathcal{D} \varsigma-q_{1} \varsigma-\mathbf{q}_{2} \cdot \delta \mathbf{u}+\overline{\mathcal{R}}_{1}[u, \varsigma] \\
\left(D_{(U, \mu, \mathbf{u})} \mathcal{F}_{2}(U, \mu, \mathbf{u})[\delta U, \delta \mu, \delta \mathbf{u}]\right) \circ X(Y)=\frac{1}{H} \mathcal{D} u+\widetilde{\mathfrak{a}} \varsigma+\mathbf{q}_{3} \cdot \delta \mathbf{u}+\widetilde{\eta} \delta \mu+\overline{\mathcal{R}_{2}}[u], \\
\left.X^{\prime}(Y)^{-1}\left(D_{(U, X, \mu, \mathbf{u})} \mathcal{F}_{3}(U, X, \rho, \nu, \mu, \mathbf{u})[\delta U, \delta X, \delta \mu, \delta \mathbf{u}]\right)\right|_{X=X(Y)}= \\
=\mathcal{D} v-\frac{1}{3}(\operatorname{div} v) \varrho+\frac{\delta \mu}{3 \widetilde{\mathfrak{a}}} \varrho+\mathbf{T}\left[X^{\prime *} \delta \mathbf{u}+\nabla u, \varsigma\right]+\overline{\mathcal{R}_{3}}[u, \varsigma, v, \delta \mu, \delta \mathbf{u}] .
\end{gathered}
$$

Here the coefficients in identities (7.6), (7.7) are given by

$$
\begin{aligned}
& K=H(\widetilde{\mathbb{Q}} \tilde{V} \cdot \tilde{V})^{1 / 2}(\widetilde{\mathbb{Q} \mathcal{D} X} \cdot \mathcal{D} X)^{-1 / 2}, \\
& q_{1}=\widetilde{\operatorname{div}_{X} V}, \quad \mathbf{q}_{2}=\widetilde{\nabla_{X} \eta}, \quad \mathbf{q}_{3}=\left(\widetilde{\nabla_{X} \psi}-\widetilde{\mathfrak{b}} \widetilde{\nabla_{X} \eta}\right) \\
& H=-\left|X^{\prime}\right|^{1 / 3}\left\{\nu \widetilde{\mathfrak{a}}(\widetilde{\mathbb{Q}} \tilde{V} \cdot \widetilde{V})^{1 / 2}\right\}^{-1 / 3}, \overline{\mathcal{G}} u=\left(\widetilde{\mathcal{G}_{\eta} \delta \phi}\right) .
\end{aligned}
$$

The linear form $\mathbf{T}$ in identity (7.8) is given by

$$
\begin{aligned}
\mathbf{T}[\boldsymbol{\xi}, \varsigma]= & \left\{\frac{1}{3 \widetilde{\mathfrak{a}}}(\overline{\mathbf{N}}[\boldsymbol{\xi}]+\overline{\mathbf{M}}[\varsigma])+\frac{1}{3 H(\widetilde{\mathbb{Q}} \widetilde{V} \cdot \widetilde{V})} \boldsymbol{\varrho} \cdot \boldsymbol{\xi}+\left[\frac{\widetilde{\mathfrak{a}}-\mu}{3 \widetilde{\mathbb{Q}} \widetilde{V} \cdot \widetilde{V}}\right] \varsigma\right\} \boldsymbol{\varrho}+ \\
& -H \mathbb{G}^{-1} \boldsymbol{\xi}+(\mathcal{D} \varsigma) \mathbb{G}^{-1} \nabla \widetilde{\eta}-\varsigma H \mathbb{G}^{-1} \nabla \widetilde{\mathfrak{b}}
\end{aligned}
$$


where

$$
\begin{aligned}
\overline{\mathbf{N}}[\boldsymbol{\xi}] & =\frac{1}{H} \mathcal{D}\left(\frac{1}{1+\left|\mathbf{q}_{2}\right|^{2}} X^{\prime-1} \mathbf{q}_{2} \cdot \boldsymbol{\xi}\right)+\mathbb{G}^{-1} \boldsymbol{\xi} \cdot \nabla \widetilde{\mathfrak{b}}, \\
\overline{\mathbf{M}}[\varsigma] & =\frac{1}{H} \mathcal{D}\left(\frac{\mathbf{q}_{2} \cdot \widehat{\nabla_{X} \mathfrak{b}}}{1+\left|\mathbf{q}_{2}\right|^{2}} \varsigma+\frac{1}{H} \frac{\mathcal{D} \varsigma}{1+\left|\mathbf{q}_{2}\right|^{2}}\right)+ \\
& +\varsigma\left(\mathbb{G}^{-1} \nabla \widetilde{\mathfrak{b}} \cdot \nabla \widetilde{\mathfrak{b}}\right)-\frac{\mathcal{D} \varsigma}{H}\left(\mathbb{G}^{-1} \nabla \widetilde{\eta} \cdot \nabla \widetilde{\mathfrak{b}}\right) .
\end{aligned}
$$

The remainders $\overline{\mathcal{R}_{i}}$ are defined by the equalities

$$
\begin{aligned}
& \overline{\mathcal{R}_{1}}[u, \varsigma]=\widetilde{\mathcal{R}_{1}}+\frac{1}{H}\left(X^{\prime-1} \mathcal{F}_{3}\right) \nabla \varsigma+(1-K) \overline{\mathcal{G}} u, \quad \overline{\mathcal{R}_{2}}[u]=-\frac{1}{H}\left(X^{\prime-1} \mathcal{F}_{3}\right) \nabla u, \\
& \overline{\mathcal{R}_{3}}[u, \varsigma, v, \delta \mu, \delta \mathbf{u}]=X^{\prime-1}\left(v \cdot \nabla_{Y} \mathcal{F}_{3}\right)+\frac{1}{3}(\operatorname{div} v) X^{\prime-1} \mathcal{F}_{3}+ \\
&-\overline{\mathbf{S}}\left[X^{\prime *} \delta \mathbf{u}+\nabla u, \varsigma, \delta \mu\right] X^{\prime-1} \mathcal{F}_{3}+ \\
&+\mathcal{R}_{S}\left[X^{\prime *} \delta \mathbf{u}+\nabla u\right]\left(\varrho-X^{\prime-1} \mathcal{F}_{3}\right)-\mathcal{R}_{V}[\varsigma],
\end{aligned}
$$

where

$$
\begin{gathered}
\mathcal{R}_{S}[\boldsymbol{\xi}, \varsigma]=\frac{1}{3 \widetilde{\mathfrak{a}}}\left(\mathcal{R}_{N}[\boldsymbol{\xi}]+\mathcal{R}_{M}[\varsigma]\right)-\frac{1}{3 H(\widetilde{\mathbb{Q}} \widetilde{V} \cdot \widetilde{V})} X^{\prime-1} \mathcal{F}_{3} \cdot \boldsymbol{\xi} \\
\mathcal{R}_{N}[\boldsymbol{\xi}]=-\frac{1}{H} X^{\prime}(Y)^{-1} \mathcal{F}_{3} \cdot \nabla\left(\frac{1}{1+\mathbf{q}_{2}^{2}} X^{\prime-1} \boldsymbol{\xi} \cdot \mathbf{q}_{2}\right), \\
\mathcal{R}_{M}[\varsigma]=-\frac{1}{H} X^{\prime-1} \mathcal{F}_{3} \cdot \nabla\left(\frac{\mathbf{q}_{2} \cdot \widetilde{\nabla_{X} \mathfrak{b}}}{1+\mathbf{q}_{2}^{2}} \varsigma+\frac{X^{\prime-1} V \cdot \nabla \varsigma}{1+\mathbf{q}_{2}^{2}}\right)+ \\
+\frac{1}{H}\left(X^{\prime-1} \mathcal{F}_{3} \cdot \nabla \varsigma\right) \mathbb{G}^{-1} \nabla \widetilde{\mathfrak{b}} \cdot \nabla \widetilde{\eta}-\frac{1}{H} \mathcal{D}\left(\frac{1}{H} \frac{X^{\prime-1} \mathcal{F}_{3} \cdot \nabla \varsigma}{1+\mathbf{q}_{2}^{2}}\right)
\end{gathered}
$$

and

$$
\mathcal{R}_{V}[\varsigma]=\left(X^{\prime-1} \mathcal{F}_{3} \cdot \nabla \varsigma\right) \mathbb{G}^{-1} \nabla \widetilde{\eta},
$$

the linear form $\overline{\mathbf{S}}$ being defined by the equality

$$
\overline{\mathbf{S}}[\boldsymbol{\xi}, \varsigma, \delta \mu]=\frac{1}{3 \widetilde{\mathfrak{a}}}(\delta \mu+\overline{\mathbf{N}}[\boldsymbol{\xi}]+\overline{\mathbf{M}}[\varsigma])+\frac{1}{3 H(\widetilde{\mathbb{Q}} \widetilde{V} \cdot \widetilde{V})} \boldsymbol{\varrho} \cdot \boldsymbol{\xi}+\left[\frac{\widetilde{\mathfrak{a}}-\mu}{3 \widetilde{\mathbb{Q}} \widetilde{V} \cdot \widetilde{V}}\right] \varsigma
$$

Remark 6 The remainders $\overline{\mathcal{R}}_{1}, \overline{\mathcal{R}}_{2}, \overline{\mathcal{R}}_{3}$, cancel identically as soon as $\mathcal{F}_{1} \equiv$ $\mathcal{F}_{2} \equiv \mathcal{F}_{3} \equiv 0$, as this results from the formula for $\mathcal{R}$ (see (4.2)) and from the identity

$$
\frac{1}{K^{2}}-1=\left(H^{2} \mathbb{Q} V \cdot V\right)^{-1}\left(2 H \mathbb{Q} V \cdot \mathcal{F}_{3}+\mathbb{Q} \mathcal{F}_{3} \cdot \mathcal{F}_{3}\right)
$$


Truncated equations. In order to apply the Implicit Function Theorem to our problem, we have to prove the invertibility of the Gateaux derivative of the nonlinear operator $\widetilde{\mathcal{F}}$, i.e. the solvability of the operator equation

$$
D_{(U, X, \mu, \mathbf{u})} \widetilde{\mathcal{F}}(U, X, \rho, \nu, \mu, \mathbf{u})[\delta U, \delta X, \delta \mu, \delta \mathbf{u}]=(f, g, \mathbf{h})
$$

By virtue of Theorem 5 this equation is equivalent to a system of linear pseudodifferential equations for periodic functions $u, \varsigma, v$ and parameters $\delta \mathbf{u}, \delta \mu$

$$
\begin{gathered}
K \overline{\mathcal{G}} u-\frac{1}{H} \mathcal{D} \varsigma-q_{1} \varsigma-\mathbf{q}_{2} \cdot \delta \mathbf{u}+\overline{\mathcal{R}}_{1}[u, \varsigma]=f \\
\frac{1}{H} \mathcal{D} u+\widetilde{\mathfrak{a}} \varsigma+\mathbf{q}_{3} \cdot \delta \mathbf{u}+\eta \delta \mu+\overline{\mathcal{R}_{2}}[u]=g \\
\mathcal{D} v-\frac{1}{3}(\operatorname{div} v) \varrho+\frac{\delta \mu}{3 \mathfrak{a}} \varrho+\mathbf{T}\left[X^{\prime *} \delta \mathbf{u}+\nabla u, \varsigma\right]+ \\
+\overline{\mathcal{R}}_{3}[u, \varsigma, v, \delta \mu, \delta \mathbf{u}]=X^{\prime-1} \mathbf{h} .
\end{gathered}
$$

In accordance to the Nash-Moser theory it is sufficient to invert the linear operator which approximates the Gateaux derivative $D \widetilde{\mathcal{F}}$ with accuracy up to the discrepancy terms $\overline{\mathcal{R}_{i}}, i=1,2,3$. Hence we can omit the operators $\overline{\mathcal{R}_{i}}$ for the study of the inverse of the differential. Thus we come to the following truncated system of operator equations

$$
\begin{gathered}
K \overline{\mathcal{G}} u-H^{-1} \mathcal{D} \varsigma-q_{1} \varsigma-\mathbf{q}_{2} \cdot \delta \mathbf{u}=f \\
H^{-1} \mathcal{D} u+\widetilde{\mathfrak{a}} \varsigma+\mathbf{q}_{3} \cdot \delta \mathbf{u}+\widetilde{\eta} \delta \mu=g, \\
\mathcal{D} v-\frac{1}{3}(\operatorname{div} v) \boldsymbol{\varrho}+\frac{\delta \mu}{3 \widetilde{\mathfrak{a}}} \boldsymbol{\varrho}+\mathbf{T}\left[X^{\prime *} \delta \mathbf{u}+\nabla u, \varsigma\right]=X^{\prime-1} \mathbf{h} .
\end{gathered}
$$

\begin{tabular}{|l|}
\hline basicsystem1 \\
\hline basicsystem2 \\
\hline basicsystem3 \\
\hline
\end{tabular}

We show that these equations are equivalent to a second order pseudodifferential equation with constant coefficients in the principal part. First we eliminate $\varsigma$ and reduce the number of equations. For doing this, note that equation (7.18) implies

$$
\varsigma=-\frac{1}{H \widetilde{\mathfrak{a}}} \mathcal{D} u-\frac{1}{\widetilde{\mathfrak{a}}} \mathbf{q}_{3} \cdot \delta \mathbf{u}-\frac{\widetilde{\eta}}{\widetilde{\mathfrak{a}}} \delta \mu+\frac{1}{\widetilde{\mathfrak{a}}} g,
$$

basicsystem2a

Substituting this expression into (7.17) and (7.19) gives the equations

$$
\begin{gathered}
K \overline{\mathcal{G}} u+\frac{1}{H^{2} \widetilde{\mathfrak{a}}} \mathcal{D}^{2} u+p_{1} \mathcal{D} u+\mathbf{p}_{2} \cdot \delta \mathbf{u}+p_{3} \delta \mu=F, \\
\mathcal{D} v-\frac{1}{3}(\operatorname{div} v) \varrho+\mathbf{Q}[\delta \mu]+\mathbf{U}[\delta \mathbf{u}]+\mathbf{Y}[u]=\mathbf{G},
\end{gathered}
$$

Here the coefficients and right hand side of equation (7.21) are defined by

$$
\begin{aligned}
& p_{1}=\frac{q_{1}}{H \tilde{\mathfrak{a}}}+\frac{1}{H} \mathcal{D}\left(\frac{1}{H \widetilde{\mathfrak{a}}}\right), \quad \mathbf{p}_{2}=\frac{1}{H} \mathcal{D}\left(\frac{1}{\tilde{\mathfrak{a}}} \mathbf{q}_{3}\right)+\frac{q_{1}}{\widetilde{\mathfrak{a}}} \mathbf{q}_{3}-\mathbf{q}_{2}, \\
& p_{3}=\frac{1}{H} \mathcal{D}\left(\frac{\widetilde{\eta}}{\widetilde{\mathfrak{a}}}\right)+\frac{\widetilde{\eta} q_{1}}{\widetilde{\mathfrak{a}}}, \quad F=f+\frac{1}{H} \mathcal{D}\left(\frac{g}{\widetilde{\mathfrak{a}}}\right)+\frac{g q_{1}}{\tilde{\mathfrak{a}}}
\end{aligned}
$$


The (2-components) linear forms $\mathbf{Q}, \mathbf{U}, \mathbf{Y}$ and right hand side of equation (7.22) are defined by the equalities

$$
\begin{aligned}
\mathbf{Q}[\delta \mu] & =\frac{\delta \mu}{3 \widetilde{\mathfrak{a}}} \boldsymbol{\varrho}-\mathbf{T}\left[0, \frac{\widetilde{\eta}}{\widetilde{\mathfrak{a}}} \delta \mu\right], \quad \mathbf{U}[\delta \mathbf{u}]=\mathbf{T}\left[X^{* *} \delta \mathbf{u},-\frac{1}{\widetilde{\mathfrak{a}}} \mathbf{q}_{3} \cdot \delta \mathbf{u}\right], \\
\mathbf{Y}[u] & =\mathbf{T}\left[\nabla u,-\frac{1}{H \widetilde{\mathfrak{a}}} \mathcal{D} u\right], \quad \mathbf{G}=X^{\prime-1} \mathbf{h}-\mathbf{T}\left[0, \frac{g}{\widetilde{\mathfrak{a}}}\right] .
\end{aligned}
$$

Our next task is to eliminate $\delta \mu$ and $\delta \mathbf{u}$ and to bring the system (7.17)-(7.19) to a triangular form. Denote by $\mathbf{u}^{\perp}$ a unit vector orthogonal to $\mathbf{u}$. Since $\mathbf{u}$ is a unit vector, we have $\mathbf{u} \cdot \delta \mathbf{u}=0$ and we have to look for the unknown vector $\delta \mathbf{u}$ in the form

$$
\delta \mathbf{u}=\delta \varpi \mathbf{u}^{\perp} .
$$

Now set

$$
\boldsymbol{\xi}=\int_{\mathbb{R}^{2} /(2 \pi \mathbb{Z})^{2}} \mathbf{Q}(1) d Y, \quad \boldsymbol{\zeta}=\int_{\mathbb{R}^{2} /(2 \pi \mathbb{Z})^{2}} \mathbf{U}\left(\mathbf{u}^{\perp}\right) d Y .
$$

rk:6.4 Remark 7 We may notice that the two vectors $\boldsymbol{\xi}$ and $\boldsymbol{\zeta}$ are not colinear. Indeed for $\psi=\eta=0, \mu=\mu_{c}, \nu=\nu_{c}, \rho=\lambda$ we have

$$
\mathfrak{a}=\mu_{c}, H=1, \mathbf{u}^{\perp}=(0,1), X^{\prime}=\mathbb{T}^{-1}, \mathbb{Q}=\mathbb{I}, V=\mathbf{u}_{0}
$$

hence

$$
\mathbf{Q}_{c}(1)=\frac{1}{3 \mu_{c}}(1, \lambda), \mathbf{U}_{c}\left(\mathbf{u}^{\perp}\right)=-\mathbb{T} \mathbf{u}_{0}^{\perp}=\left(-\tau_{1}, \lambda \tau_{2}\right)
$$

and

$$
\left|(1, \lambda) \times\left(-\tau_{1}, \lambda \tau_{2}\right)\right|=\lambda\left(\tau_{1}+\tau_{2}\right) \neq 0 .
$$

Since the vector field $\mathcal{D} v-3^{-1}(\operatorname{div} v) \varrho$ in $(7.22)$ has a zero average over the period, the average of (7.22) leads to the equation

$$
\delta \mu \boldsymbol{\xi}+\delta \varpi \boldsymbol{\zeta}=-\int_{\mathbb{R}^{2} /(2 \pi \mathbb{Z})^{2}} \mathbf{Y}[u] d Y+\int_{\mathbb{R}^{2} /(2 \pi \mathbb{Z})^{2}} \mathbf{G} d Y
$$

which gives

$$
\delta \mu=\boldsymbol{\Xi}_{1}[u, \mathbf{G}], \quad \delta \varpi=\boldsymbol{\Xi}_{2}[u, \mathbf{G}],
$$

where the linear functionals $\boldsymbol{\Xi}_{j}$ are defined by the equalities

$$
\begin{aligned}
& \boldsymbol{\Xi}_{1}[u, \mathbf{G}]=\frac{1}{\boldsymbol{\xi} \cdot \boldsymbol{\zeta}^{\perp}} \boldsymbol{\zeta}^{\perp} \cdot \int_{\mathbb{R}^{2} /(2 \pi \mathbb{Z})^{2}}(-\mathbf{Y}[u]+\mathbf{G}) d Y, \\
& \boldsymbol{\Xi}_{2}[u, \mathbf{G}]=\frac{1}{\boldsymbol{\xi}^{\perp} \cdot \boldsymbol{\zeta}} \boldsymbol{\xi}^{\perp} \cdot \int_{\mathbb{R}^{2} /(2 \pi \mathbb{Z})^{2}}(-\mathbf{Y}[u]+\mathbf{G}) d Y .
\end{aligned}
$$

Substituting these relations into (7.21), (7.22) we obtain a triangular system of operator equations

$$
\begin{aligned}
K \overline{\mathcal{G}} u+\frac{1}{H^{2} \mathfrak{a}} \mathcal{D}^{2} u & +p_{1} \mathcal{D} u+\boldsymbol{\Xi}_{1}[u, \mathbf{0}] p_{3}+\boldsymbol{\Xi}_{2}[u, \mathbf{0}] \mathbf{p}_{2} \cdot \mathbf{u}^{\perp} \\
& =F-\boldsymbol{\Xi}_{2}[0, \mathbf{G}] \mathbf{p}_{2} \cdot \mathbf{u}^{\perp}-\boldsymbol{\Xi}_{1}[0, \mathbf{G}] p_{3}
\end{aligned}
$$




$$
\mathcal{D} v-\frac{1}{3}(\operatorname{div} v) \varrho=\mathbf{G}-\boldsymbol{\Xi}_{1}[u, \mathbf{G}] \mathbf{Q}(1)-\boldsymbol{\Xi}_{2}[u, \mathbf{G}] \mathbf{U}\left(\mathbf{u}^{\perp}\right)-\mathbf{Y}[u]
$$

Remark 8 By construction of the functionals $\boldsymbol{\Xi}_{j}$, the right hand side of equation (7.28) has automatically zero mean value over the fundamental cell of the periodic lattice.

Our further considerations are based on the analysis of system (7.27)(7.28). Notice that after solving the first equation (7.27) for the function $u$, we can easily find the second unknown $v$ inverting the operator $\mathcal{D}-3^{-1}(\operatorname{div} \cdot) \varrho$. Hence, the main task is to prove the solvability of equation (7.27). It is now sufficient to prove the solvability, in the Sobolev space of odd $2 \pi$-periodic functions of the linear operator equation

$$
K \overline{\mathcal{G}} u+\frac{1}{H^{2} \widetilde{\mathfrak{a}}} \mathcal{D}^{2} u+p_{1} \mathcal{D} u+\mathcal{H}_{-1} u=F,
$$

where

$$
\mathcal{H}_{-1} u=\boldsymbol{\Xi}_{1}[u, \mathbf{0}] p_{3}+\boldsymbol{\Xi}_{2}[u, \mathbf{0}] \mathbf{p}_{2} \cdot \mathbf{u}^{\perp}
$$

maps $H_{o}^{s}$ to $H_{o}^{s+1}$ for any $s \geq 2$, with a norm of order $O(|\varepsilon|)$. The proof of the invertibility of (7.29) occupies sections 9 to 11 . First of all we clarify the structure of the linear operators involved in the main equation (7.29). The most suitable tool for this is the theory of pseudodifferential operators in Sobolev spaces, and in the next section we recall basic definitions and results.

\section{Pseudodifferential operators. Auxiliary propositions.}

Pseudodifferential operators. Basic definitions. We deal with the class of integro-differential operators on a two-dimensional torus having the representation

$$
\mathfrak{A} u(Y)=\frac{1}{2 \pi} \sum_{\mathbf{k} \in \mathbb{Z}^{2}} e^{i \mathbf{k} \cdot Y} A(Y, \mathbf{k}) \widehat{u}_{\mathbf{k}} .
$$

The operator $\mathfrak{A}$ is completely characterized by the function $A(Y, \mathbf{k})$ - the symbol of $\mathfrak{A}$. We use Gothic capitals for pseudodifferential operators and Roman capitals for their symbols. Recall that $\mathfrak{A}$ is a pseudodifferential operator, if its symbol satisfies the condition

condA Condition 6 There are integers $l>0, m \geq 0$ and a real $r$ named the order of the operator $\mathfrak{A}$ so that

$$
|\mathfrak{A}|_{m, l}^{r}=\|A(\cdot, 0)\|_{\mathcal{C}^{l}}+\sup _{k \in \mathbb{Z}^{2} \backslash\{0\}} \sup _{|\alpha| \leq m}|k|^{|\alpha|-r}\left\|\partial_{k}^{\alpha} A(\cdot, k)\right\|_{\mathcal{C}^{l}}<\infty .
$$

The following lemma immediately follows from the definition and the Parceval identity, see Proposition F.1 in [16]. 
lemma10.1 Lemma 8 Let $|\mathfrak{A}|_{0, l}^{t}<\infty$ and $0 \leq s \leq l-3, s+t \geq 0$. Then there is $a$ constant $c$, depending on $s, r$ and $l$ only, so that for all $u \in H^{s+r}$

$$
\|\mathfrak{A} u\|_{s} \leq\left(|\mathfrak{A}|_{0, l}^{t}\|u\|_{t}+|\mathfrak{A}|_{0,3}^{r}\|u\|_{t+s}\right) .
$$

If $t<0$, then the norm $\|u\|_{t}$ should be replaced with $\|u\|_{0}$.

An important example of such operators is the first order pseudodifferential operator $\mathfrak{G}_{1}^{(0)}=(-\Delta)^{1 / 2}$ with the symbol $($ see $(9.6)$ )

$$
G_{1}^{(0)}(\mathbf{k})=(\mathbb{A} \widetilde{\mathbf{k}} \cdot \widetilde{\mathbf{k}})^{1 / 2} \equiv\left|\mathbb{A}^{1 / 2} \widetilde{\mathbf{k}}\right|, \quad \widetilde{\mathbf{k}}=\left(k_{1}+\rho k_{2}, k_{2}\right)
$$

associated with a fixed positive symmetric matrix $\mathbb{A}$, and the second order pseudodifferential operator

$$
\mathfrak{L}=\nu \mathcal{D}^{2}+(-\Delta)^{1 / 2}, \text { where } \mathcal{D}=\partial_{y_{1}}+\rho \partial_{y_{2}} .
$$

The symbol of $\mathfrak{L}$ is determined by the equality

$$
L(\mathbf{k}):=\left|\mathbb{A}^{1 / 2} \widetilde{\mathbf{k}}\right|-\nu\left(k_{1}+\rho k_{2}\right)^{2}
$$

We also define "the inverse operators" $(-\Delta)^{-1 / 2}$ and $\mathcal{D}^{-1}$ by the equalities

$$
\begin{aligned}
\left(\left(-\widehat{\Delta)^{-1} / 2} u\right)_{\mathbf{k}}\right. & =\left|\mathbb{A}^{1 / 2} \widetilde{\mathbf{k}}\right|^{-1} \widehat{u}_{\mathbf{k}} \text { for } \mathbf{k} \neq 0, \quad\left(\left(-\widehat{\Delta)^{-1} / 2} u\right)_{\mathbf{0}}=0\right. \\
\left(\widehat{\mathcal{D}^{-1} u}\right)_{\mathbf{k}} & =\left(i k_{1}+i \rho k_{2}\right)^{-1} \widehat{u}_{\mathbf{k}} \text { for } \mathbf{k} \neq 0, \quad\left(\widehat{\mathcal{D}^{-1} u}\right)_{\mathbf{0}}=0 .
\end{aligned}
$$

They are real inverses of $(-\Delta)^{1 / 2}$ and $\mathcal{D}$ on the subspace $H^{0}$ of all function orthogonal to constants. It is important to note that the operator $\mathcal{D}^{-1}$ is not pseudodifferential.

Further we deal with the special class of zero order pseudodifferential operators $\mathfrak{A}$ with symbols having the form of a composition $A(Y, \boldsymbol{\xi}(\mathbf{k}))$, where the vector field $\boldsymbol{\xi}(\mathbf{k})$ is defined by

$$
\boldsymbol{\xi}=\left(\xi_{1}, \xi_{2}\right)=\frac{\widetilde{\mathbf{k}}}{\left|\mathbb{A}^{1 / 2} \widetilde{\mathbf{k}}\right|}, \quad \text { for } \mathbf{k} \neq 0 \text { and } \boldsymbol{\xi}(0)=0
$$

The metric properties of such operators are characterized by the norm

$$
|A|_{l}=\sup _{|\alpha| \leq 6 c^{-1} \leq|\boldsymbol{\xi}| \leq c} \sup _{\mid \partial_{\boldsymbol{\xi}} A(\cdot, \boldsymbol{\xi}) \|_{C^{l}}<\infty}
$$

By abuse of notation, further we simply write $\boldsymbol{\xi}$ instead of $\boldsymbol{\xi}(\mathbf{k})$. 
Dirichlet-Neumann operator. In our framework, the most important fact is that the Dirichlet-Neumann operator is the sum of a pseudodifferential operator and a smoothing remainder. This results from the following theorem, which will be used throughout the paper (see [16] Theorem 3.5).

Neumann Theorem 7 Suppose that there is an integer $r$, with $10 \leq r$, and a positive $\varepsilon>0$, such that

$$
\|\widetilde{\eta}\|_{\mathcal{C}^{r}\left(\mathbb{R}^{2} /(2 \pi \mathbb{Z})^{2}\right)}+\left\|X-\mathbb{T}^{-1}\right\|_{\mathcal{C}^{r}\left(\mathbb{R}^{2} /(2 \pi \mathbb{Z})^{2}\right)} \leq \varepsilon
$$

Then there is $\varepsilon_{0}>0$ depending only on $r$ such that for all $0<\varepsilon<\varepsilon_{0}$ and $2 \pi$ periodic sufficiently smooth function $u$, the operator $\overline{\mathcal{G}}$ has the representation

$$
\overline{\mathcal{G}} u=\mathcal{G}_{1} u+\mathcal{G}_{0} u+\mathcal{G}_{-1} u .
$$

Here $\mathcal{G}_{1}$ is a first order pseudodifferential operator with the symbol

$$
G_{1}(Y, \mathbf{k})=\left(\frac{\operatorname{det} \mathbb{G}}{\operatorname{det} X^{\prime 2}}\right)^{1 / 2}\left(\mathbb{G}^{-1} \mathbf{k} \cdot \mathbf{k}\right)^{1 / 2},
$$

$\mathcal{G}_{0}$ is a zero order pseudodifferential operator with symbol

$$
\tilde{G}_{0}=\operatorname{Re} \tilde{G}_{0}+i \operatorname{Im} \tilde{G}_{0}
$$

$$
\begin{array}{r}
\operatorname{Re} \tilde{G}_{0}=\frac{\operatorname{det} \mathbb{G}}{2 \operatorname{det} X^{\prime 2}}\left[\frac{1}{\mathbb{G}^{-1} \mathbf{k} \cdot \mathbf{k}} P(Y, \mathbf{k})+\operatorname{div}\left(\mathbb{G}^{-1} \nabla \widetilde{\eta}\right)\right] \\
\operatorname{Im} \tilde{G}_{0}=-\frac{1}{2}\left(\frac{\operatorname{det} \mathbb{G}}{\operatorname{det} X^{\prime 2}}\right)^{1 / 2} \operatorname{div}\left[\nabla_{\mathbf{k}}\left(\mathbb{G}^{-1} \mathbf{k} \cdot \mathbf{k}\right)^{1 / 2}\right] .
\end{array}
$$

Here the quadratic form $P(Y, \cdot)$ is given by

$$
P(Y, \mathbf{k})=\frac{1}{2} \nabla\left(\mathbb{G}^{-1} \mathbf{k} \cdot \mathbf{k}\right) \cdot\left(\mathbb{G}^{-1} \nabla \widetilde{\eta}\right)-\mathbb{G}^{-1} \mathbf{k} \cdot \nabla\left(\mathbb{G}^{-1} \mathbf{k} \cdot \nabla \widetilde{\eta}\right) .
$$

The symbol of the zero order operator $\mathcal{G}_{0}$ can also be written in the standard form

$$
\check{G}_{0}(Y, \boldsymbol{\xi}(\mathbf{k}))=: \tilde{G}_{0}\left(Y ; \xi_{1}-\rho \xi_{2}, \xi_{2}\right),
$$

and the symbol $\check{G}_{0}$ admits the estimate

$$
\left|\check{G}_{0}\right|_{r-2} \leq c \varepsilon
$$

while the linear operator $\mathcal{G}_{-1}$ satisfies

$$
\left\|\mathcal{G}_{-1} u\right\|_{s} \leq c \varepsilon\|u\|_{s-1} \quad \text { for } 1 \leq s \leq r-9
$$

Moreover, if a function $\widetilde{\eta}(Y)$ is even and the function $X(Y)$ is odd in $Y, \mathcal{G}_{i} u$ is even for even $u$ and odd for odd $u$. 
Commutators and Compositions. It is well known that the principal part of the composition $\mathfrak{A} \mathfrak{B}$ of pseudodifferential operators $\mathfrak{A}$ and $\mathfrak{B}$ is also a pseudodifferential operator with the symbol $A B$, and the order of the commutator $\mathfrak{A} \mathfrak{B}-\mathfrak{B A}$ is less that the sum of orders of the operators $\mathfrak{A}$ and $\mathfrak{B}$. The following lemma, (see Proposition F.1 in [16]) gives the explicit formulation of this result.

lemma11.3 Lemma 9 Let $\mathfrak{A}$ and $\mathfrak{W}$ be zero order pseudodifferential operators so that for some non-negative integer $l,|A|_{l}+|W|_{l}<\infty$, where the norm $|\cdot|_{l}$ of the symbol $A(Y, \boldsymbol{\xi})$ is determined by

$$
|A|_{l}=\sup _{|\alpha| \leq 6} \sup _{c^{-1} \leq|\boldsymbol{\xi}| \leq c}\left\|D_{\boldsymbol{\xi}}^{\alpha} A(\cdot, \boldsymbol{\xi})\right\|_{C^{l}} .
$$

Then for any $u \in H^{s}$ with $s \in[0, l-5]$, we have

$$
\|\mathfrak{A} u\|_{s} \leq c\left(|A|_{3}\|u\|_{s}+|A|_{l}\|u\|_{0}\right)
$$

The composition of the operators $\mathfrak{A}$ and $\mathfrak{W}$ has the representation

$$
\mathfrak{A W}=(\mathfrak{A W})_{0}+\mathfrak{D}_{1}^{(\mathfrak{A} \mathfrak{W})}=(\mathfrak{A W})_{0}+(\mathfrak{A W})_{1}+\mathfrak{D}_{2}^{(\mathfrak{A} \mathfrak{W})}
$$

where the pseudodifferential operators $(\mathfrak{A W})_{0}$ and $(\mathfrak{A W})_{1}$ have the symbols

$$
A(Y, \boldsymbol{\xi}) W(Y, \boldsymbol{\xi}) \quad \text { and }=-i \nabla_{k} A(Y, \boldsymbol{\xi}) \cdot \nabla_{Y} W(Y, \boldsymbol{\xi}) .
$$

Here we adopt the convention that $\nabla_{k} W(Y, \boldsymbol{\xi}(\mathbf{k}))=0$ when $\mathbf{k}=0$. For any $s \in[0, l-7]$, the remainders admit the estimates

$$
\left\|\mathfrak{D}_{i}^{(\mathfrak{A} \mathfrak{W})} u\right\|_{s+i} \leq\left. c|A|_{6}|| W\right|_{6}\|u\|_{s}+c\left(\left.|A|_{l}|| W\right|_{6}+|A|_{6}|W|_{l}\right)\|u\|_{0}
$$

The commutator $\left[(-\Delta)^{1 / 2}, \mathfrak{W}\right]$ has the representation

$$
(-\Delta)^{1 / 2} \mathfrak{W}-\mathfrak{W}(-\Delta)^{1 / 2}=\left[(-\Delta)^{1 / 2}, \mathfrak{W}\right]_{1}+\mathfrak{D}_{2}^{\left[(-\Delta)^{1 / 2}, W\right]},
$$

where the symbol of the operator $\left[(-\Delta)^{1 / 2}, \mathfrak{W}\right]_{1}$ is equal to

$$
-i \nabla_{k}(\mathbb{A} \tilde{\mathbf{k}} \cdot \widetilde{\mathbf{k}})^{1 / 2} \cdot \nabla_{Y} W(Y, \boldsymbol{\xi}),
$$

when $\mathbf{k} \neq 0$, and 0 otherwise. For $s \in[0, l-7]$, the remainder admits the estimate

$$
\left\|\mathfrak{D}_{2}^{\left[(-\Delta)^{1 / 2}, \mathfrak{W}\right]} u\right\|_{s+1} \leq c|W|_{6}\|u\|_{s}+c|W|_{l} \mid\|u\|_{0}
$$


Resonance projection. For fixed positive $\nu$ we denote by $\mathcal{N} \subset \mathbb{Z}^{2}$ the set

$$
\mathcal{N}=\left\{\mathbf{k} \in \mathbb{Z}^{2}:\left(k_{1}+\rho k_{2}\right)^{2}>(4 \nu)^{-1}\left|\mathbb{A}^{1 / 2} \widetilde{\mathbf{k}}\right|\right\}
$$

Denote by $\Pi$ the projection defined in terms of the Fourier transform by the equalities

$$
(\widehat{\Pi u})_{\mathbf{k}}=\widehat{u}_{\mathbf{k}} \text { for } \mathbf{k} \in \mathcal{N}, \quad(\widehat{\Pi u})_{\mathbf{k}}=0 \text { otherwise }
$$

An inherent characteristic of this projection which immediately follows from its definition is that the operators $\mathfrak{L}^{-1}(\mathbf{I}-\Pi)$ and $\mathcal{D}^{-1} \Pi$ are bounded and admit the estimates

$$
\begin{aligned}
& \left\|\mathfrak{L}^{-1}(\mathbf{I}-\Pi) u\right\|_{s+1} \leq c(s)\|u\|_{s} \text { for all } u \in H_{o}^{s}, \quad 0 \leq s<\infty \\
& \left\|\mathcal{D}^{-1} \Pi u\right\|_{s+1 / 2} \leq c(s)\|u\|_{s} \text { for all } u \in H^{s}, \quad 0 \leq s<\infty
\end{aligned}
$$

The operator $\Pi$ enjoys many remarkable properties the most important of which are given by the following lemma proved in Appendix E.

Lemma 10 Let $\mathfrak{H}: H_{o}^{s} \rightarrow H_{o}^{s}$ be a zero order pseudodifferential operator with an integer $r>5$ such that $|H|_{r} \leq c_{H} \varepsilon$. Then for any $s \in[0, r-5)$ there are a constant $c$, depending only on $s$ and $c_{H}$, and bounded operators $\mathfrak{Y}, \mathfrak{Z}: H_{o}^{s} \rightarrow H_{o}^{s+1}$ so that $[\Pi, \mathfrak{H}]=\mathfrak{Y} \mathfrak{L}+\mathfrak{Z}$ and

$$
\|\mathfrak{Y} u\|_{s+1}+\|\mathfrak{Z} u\|_{s+1} \leq c \varepsilon\|u\|_{s} \text { for all } u \in H_{o}^{s} .
$$

Contraction mapping principle. The following simple corollary of the contraction mapping principle will be used throughout the rest of the paper.

Lemma 11 Let $0 \leq s$ and for all $u \in H_{o}^{s}$ the operator $\mathfrak{U}_{i}$ satisfy the inequalities

$$
\left\|\mathfrak{U}_{i} u\right\|_{s} \leq c_{1} \varepsilon\|u\|_{s}, \quad i=1, . ., n
$$

Then there is $\varepsilon_{0}>0$, depending only on $c_{1}$ and $n$ so that for all $\varepsilon \in\left[0, \varepsilon_{0}\right]$ the operator

$$
\mathfrak{W}_{n}=\prod_{i=1}^{n}\left(\mathbf{I}+\mathfrak{U}_{i}\right)^{j_{i}}, \quad j_{i}= \pm 1 .
$$

satisfies the inequalities

$$
\left\|\left(\mathfrak{W}_{n}^{ \pm 1}-\mathbf{I}\right) u\right\|_{s} \leq c \varepsilon\|u\|_{s}
$$




\section{Normal form of the operator equation}

This section occupies a highly important place in the paper. It is devoted to the study of the pseudodifferential operator

$$
K \overline{\mathcal{G}}+\frac{1}{H^{2} \widetilde{\mathfrak{a}}} \mathcal{D}^{2}+p_{1} \mathcal{D}+\mathcal{H}_{-1}
$$

which forms the basic equation (7.29). The aim is to show that this operator is similar, up to some lower-order unessential terms, to the linear operator with " constant coefficients" $(\mathfrak{L}+\mathfrak{V}) \Pi+\mathfrak{L}(\mathbf{I}-\Pi)$, where the pseudodifferential operator $\mathfrak{L}$ and the resonance projection $\Pi$ are defined by formulae $(8.3)$ and (8.26), $\mathfrak{V}$ is a zero-order pseudodifferential operator with a symbol $V(\xi)$. This result completed by estimates of the $\mathfrak{L}$ - resolvent, displayed in next section, makes it possible to reduce equation (7.29) to a Fredholm-type operator equation.

Before to formulate the results we introduce notations which will be used throughout this section. First of all we specify the matrix $\mathbb{A}$ in the definition of $\mathfrak{L}$, and the symbol $V(\boldsymbol{\xi})$. To this end we introduce the temporary notation for differential operators

$$
\boldsymbol{\partial}=\mathbb{S} \nabla_{Y}, \quad \boldsymbol{\partial}^{*}=\operatorname{div}\left(\mathbb{S}^{*} \cdot\right) \text { where } \mathbb{S}=\left(\begin{array}{ll}
1 & \rho \\
0 & 1
\end{array}\right) .
$$

In particular, we have $\widetilde{\mathbf{k}}=\mathbb{S} \mathbf{k}$. Let us introduce the matrix $\mathbb{G}_{1}$ with the entries

$$
G_{1,22}=1, G_{1,12}=\frac{-g_{12}-\rho g_{22}}{g_{11}+2 g_{12} \rho+g_{22} \rho^{2}}, G_{1,11}=\frac{g_{22}}{g_{11}+2 g_{12} \rho+g_{22} \rho^{2}},
$$

composi4a

where $\mathbb{G}=\left(g_{i j}\right)_{2 \times 2}$ is the first fundamental matrix of the free surface (see (5.5)) in the parametrization $x=(X(Y), \eta(X(Y)))$, and a function

$$
\mathfrak{g}(Y)=\left|X^{\prime}(Y)\right|^{2 / 3}\left(\mathfrak{a} \nu(\mathbb{Q} V \cdot V)^{-1}\right)^{1 / 3} \circ X(Y),
$$

mathfrakg

and introduce also pseudodifferential operators $\mathfrak{G}_{i}, i=1,0$ with symbols

$$
J_{1}(Y, \mathbf{k})=\left(\mathbb{G}_{1} \tilde{\mathbf{k}} \cdot \widetilde{\mathbf{k}}\right)^{1 / 2}, \quad J_{0}(Y, \boldsymbol{\xi}(\mathbf{k}))=\mathfrak{g} K \check{G}_{0}(Y, \boldsymbol{\xi})
$$

where the symbol $\check{G}_{0}(Y, \boldsymbol{\xi})$ is given by (8.16) and (8.12). We define the average $\mathbb{A}$ of $\mathbb{G}_{1}$ and the deviation $\mathbb{B}$ as follows

$$
\begin{gathered}
\mathbb{A}=\left(\mathbb{A}_{i j}\right)_{2 \times 2}, \quad \mathbb{A}_{i j}=\frac{1}{4 \pi^{2}} \int_{\mathbb{R}^{2} /(2 \pi \mathbb{Z})^{2}} G_{1, i j}(Y) d Y, \\
\mathbb{B}=\left(\mathbb{B}_{i j}\right)_{2 \times 2}, \quad \mathbb{B}_{i j}=G_{1, i j}-\mathbb{A}_{i j} .
\end{gathered}
$$

We introduce two complex-valued periodic functions $\tilde{A}(Y)$ and $\tilde{W}(Y)$

$$
\begin{aligned}
& \tilde{A}:=\operatorname{Re} \tilde{A}+i \operatorname{Im} \tilde{A}=\mathfrak{g} p_{1}(Y)-i \mathbb{B}_{12}(Y), \\
& \tilde{W}:=\operatorname{Re} \tilde{W}+i \operatorname{Im} \tilde{W}=\exp \left\{(2 \nu)^{-1} \mathcal{D}^{-1} \tilde{A}\right\}-1 .
\end{aligned}
$$


Introduce also two basic elementary pseudodifferential operators $\mathfrak{A}$ and $\mathfrak{W}$ with the symbols

$$
A(Y, \boldsymbol{\xi}):=\operatorname{Re} \tilde{A}+i \xi_{2} \operatorname{Im} \tilde{A}, \quad W(Y, \boldsymbol{\xi}):=\operatorname{Re} \tilde{W}+i \xi_{2} \operatorname{Im} \tilde{W},
$$

and a zero order operator $\mathfrak{B}$ with the symbol

$$
B(Y, \boldsymbol{\xi})=Q_{0}(Y, \boldsymbol{\xi})\left(1+i \xi_{1} A(Y, \boldsymbol{\xi})\right)+J_{0}(Y, \boldsymbol{\xi}) .
$$

Here the symbol $Q_{0}(Y, \boldsymbol{\xi})$ is defined by

$$
\begin{aligned}
& \nu Q_{0}(Y, \boldsymbol{\xi})=\frac{1}{2} \mathbb{B}_{11}(Y)+Q_{01}(Y, \boldsymbol{\xi}), \\
& Q_{01}(Y, \boldsymbol{\xi})=-\left\{\left(2 \mathbb{B}_{12}(Y) \xi_{2}+\mathbb{B}_{11}(Y) \xi_{1}\right)^{2}\right\} \times \\
& \times\left\{4+4 \mathbb{B}_{12}(Y) \xi_{1} \xi_{2}+2 \mathbb{B}_{11}(Y) \xi_{1}^{2}+4\left[1+2 \mathbb{B}_{12}(Y) \xi_{1} \xi_{2}+\mathbb{B}_{11}(Y) \xi_{1}^{2}\right]^{1 / 2}\right\}^{-1} .
\end{aligned}
$$

Next, introduce zero-order pseudodifferential operators $\mathfrak{B}_{0}, \mathfrak{B}_{1}$ and $\mathfrak{E}$ with the symbols

$$
B_{0}(Y, \boldsymbol{\xi})=[1+W(Y, \boldsymbol{\xi})]^{-1}\left[B(Y, \boldsymbol{\xi})+B_{1}(Y, \boldsymbol{\xi})+E(Y, \boldsymbol{\xi})\right],
$$

in which

$$
\begin{aligned}
& B_{1}=\nu \mathcal{D}^{2} W+A \mathcal{D} W+B W-i \boldsymbol{\partial} W \cdot \mathbb{A} \boldsymbol{\xi}+ \\
& +\xi_{1}\left[\nabla_{\xi} A \cdot \mathbb{A} \boldsymbol{\partial} W-\left(\nabla_{\xi} A \cdot \mathbb{A} \boldsymbol{\xi}\right)(\boldsymbol{\partial} W \cdot \mathbb{A} \boldsymbol{\xi})\right] \\
& E(Y, \boldsymbol{\xi})=\nu^{-1} i \operatorname{Im} \tilde{A} \operatorname{Im} \tilde{W}\left(2 A_{12} \xi_{2}+A_{11} \xi_{1}\right) .
\end{aligned}
$$

Finally set

$$
V(\boldsymbol{\xi})=\frac{1}{4 \pi^{2}} \int_{0}^{2 \pi} \int_{0}^{2 \pi} B_{0}(Y, \boldsymbol{\xi}) d Y .
$$

Now we can formulate the main result of this section. Assume that the $2 \pi$ periodic function $\widetilde{\eta}$ and the mapping $X(Y)$ satisfy the following conditions.

(H.1) There is an integer $r \geq 26$ and positive constants $\epsilon, c_{0}$ such that

$$
\begin{aligned}
& c_{0}^{-1} \leq \nu \leq c_{0}, \quad c_{0}^{-1} \leq \rho \leq c_{0}, \\
& \|\eta\|_{\mathcal{C}^{r}\left(\mathbb{R}^{2} / \Gamma\right)}+\|\psi\|_{\mathcal{C}^{r}\left(\mathbb{R}^{2} / \Gamma\right)}+\left\|X-\mathbb{T}^{-1}\right\|_{\mathcal{C}^{r}\left(\mathbb{R}^{2} /(2 \pi \mathbb{Z})^{2}\right)} \leq \epsilon^{1 / 2} .
\end{aligned}
$$

Here $\nu$ and $\rho$ are parameters occuring in the definitions of $\mathfrak{L}$ and $\mathcal{D}$.

(H.2) The rotation number $\rho$ satisfies the Diophantine condition

$$
\left|k_{1}+\rho k_{2}\right|^{-1} \leq c_{0}|\mathbf{k}|^{4 / 3} \text { for all } \mathbf{k} \in \mathbb{Z}^{2} \backslash\{0\} .
$$

diophantine

Note that this condition is fulfilled for a.e. $\rho \in \mathbb{R}$.

Remark 9 In the following, $\epsilon$ is a shortcut notation for a small quantity of order $O\left(|\rho-\lambda|+\left|\nu-\nu_{c}\right|\right)$, due to our choice of parameters. 
Theorem 8 Let $\eta, \psi, X, \rho, \nu$, and exponent $r$ satisfy conditions (H.1)(H.2). Furthermore, assume that

$$
2 \leq s \leq r-24
$$

Then there is $\epsilon_{0}>0$, depending only on $r$, the lattice $\Gamma$, and on the constant $c_{0}>0$ in (H.1)-(H.2), with the following properties. For any $\epsilon \in\left(0, \epsilon_{0}\right)$, there are bounded operators $\mathfrak{S}, \mathfrak{T}: H_{o}^{s} \mapsto H_{o}^{s}, \mathfrak{F}: H_{o}^{s} \mapsto H_{o}^{s+1}, \mathfrak{P}: H_{o}^{s} \mapsto H_{o}^{s}$ so that

$$
\begin{aligned}
& \mathfrak{S}\left\{\mathfrak{g}\left(K \overline{\mathcal{G}}+\frac{1}{H^{2} \widetilde{\mathfrak{a}}} \mathcal{D}^{2}+p_{1} \mathcal{D}+\mathcal{H}_{-1}\right)\right\} \mathfrak{T}= \\
& =\Pi(\mathfrak{L}+\mathfrak{V}+\mathfrak{F}) \Pi+(\mathbf{I}-\Pi)(\mathfrak{L}+\mathfrak{P}) .
\end{aligned}
$$

Moreover, for any $u \in H_{o}^{s}$ the operators $\mathfrak{V}, \mathfrak{F}, \mathfrak{P}$, and $\mathfrak{S}, \mathfrak{T}$ admit the estimates

$$
\begin{gathered}
\|\mathfrak{V} u\|_{s} \leq c \epsilon\|u\|_{s},\|\mathfrak{P} u\|_{s}+\|\mathfrak{F} u\|_{s+1} \leq c \epsilon^{1 / 2}\|u\|_{s}, \\
\left\|\left(\mathfrak{S}^{ \pm 1}-\mathbf{I}\right) u\right\|_{s}+\left\|\left(\mathfrak{T}^{ \pm 1}-\mathbf{I}\right) u\right\|_{s} \leq c \epsilon^{1 / 2}\|u\|_{s},
\end{gathered}
$$

where the symbol $V(\boldsymbol{\xi})$ of the zero order operator $\mathfrak{V}$ is given by formula (9.15). If, in addition for $l \geq 0$

$$
E_{l}=\|\eta\|_{\mathcal{C}^{r+l}\left(\mathbb{R}^{2} / \Gamma\right)}+\|\psi\|_{\mathcal{C}^{r+l}\left(\mathbb{R}^{2} / \Gamma\right)}+\left\|X-\mathbb{T}^{-1}\right\|_{\mathcal{C}^{r+l}\left(\mathbb{R}^{2} /(2 \pi \mathbb{Z})^{2}\right)},
$$

then

$$
\begin{gathered}
\|\mathfrak{P} u\|_{s+l}+\|\mathfrak{F} u\|_{s+1+l} \leq c \epsilon^{1 / 2}\|u\|_{s+l},+c E_{l}\|u\|_{s} \\
\left\|\left(\mathfrak{S}^{ \pm 1}-\mathbf{I}\right) u\right\|_{s+l}+\left\|\left(\mathfrak{T}^{ \pm 1}-\mathbf{I}\right) u\right\|_{s+l} \leq c \epsilon^{1 / 2}\|u\|_{s+l}+c E_{l}\|u\|_{s}
\end{gathered}
$$

where the constant $c$ depends only on $s$ and $c_{0}$.

The proof of this theorem occupies the rest of the section. In order to avoid repetitions we give the proof of the theorem only in the case $l=0$. It follows from the tame estimates for compositions and commutators in Lemma 9 that the same proof works for $l>0$, see [16]. We split the proof into four propositions and one technical lemma. First of all we prove the auxiliary Lemma 12, which gives the estimates for the symbols $A, B, B_{0}$ and $V$. Next we prove the preliminary Proposition 1 and reduce the operator (9.1) to a canonical form with the operator $\mathfrak{L}+\mathfrak{A D}+\mathfrak{B}$ in the principal part. The proof of these results occupies Subsection 9.1.

Next propositions correspond to three steps in the so called descent method [16]. We use the scheme proposed in [16], to make the first step of the descend method and eliminate the first order term $\mathfrak{A D}$ in $\mathfrak{L}+\mathfrak{A D}+\mathfrak{B}$. This result is given by Proposition 2 in Subsection 9.2. The justification of the second and third steps in the descent method uses explicitly, in the formulation of the new linear operator, the inversion of the differential operator $\mathcal{D}$ which would imply a loss of regularity. We cope with this difficulty by using Lemma 10 on the commutators of the resonance projection. The corresponding results are given in Propositions 3 and 4. It is worthy to note that our proof is conceptually algebraic. In particular, we do not require the existence of the $\mathfrak{L}$ resolvent. The only metric restriction is the diophantine condition (H.2). 
9.1 Preliminaries

Symbols estimates. Our first result is the following auxiliary lemma which gives the estimates for coefficients of operator (9.1) and symbols (9.8)-(9.15).

Lemma 12 Under the assumptions of Theorem 8, there is $\epsilon_{1}>0$ and constants $c_{1}, a_{0}$, depending only on the exponent $r$, the lattice $\Gamma$, and the constant $c_{0}$ in (H.1)-(H.2), with the following properties. For any $\epsilon \in\left(0, \epsilon_{1}\right)$, the matrix $\mathbb{A}$ and the symbols (9.8)-(9.15) satisfy the inequalities

$$
\begin{gathered}
a_{0}^{-1}|\mathbf{k}| \leq(\mathbb{A} \widetilde{\mathbf{k}} \cdot \widetilde{\mathbf{k}})^{1 / 2} \leq a_{0}|\mathbf{k}| \text { for all } \widetilde{\mathbf{k}} \in \mathbb{Z}^{2}, \\
|A|_{r-3}+|W|_{r-6}<c \epsilon^{1 / 2},\left|Q_{0}\right|_{r-1}+|B|_{r-3}<c \epsilon^{1 / 2}, \\
\left|B_{i}\right|_{r-8}+|E|_{r-6}<c \epsilon^{1 / 2},|V(\boldsymbol{\xi})| \leq c \epsilon^{1 / 2} .
\end{gathered}
$$

Moreover, for all $s \in[0, r-10]$ and $u \in H_{o}^{s}$,

$$
\left\|\mathfrak{g}-\mathfrak{g}^{(0)}\right\|_{C^{s+1}} \leq c \epsilon^{1 / 2}, \quad\left\|\mathfrak{G}_{-1} u\right\|_{s+1}<c \epsilon^{1 / 2}\|u\|_{s},
$$

where $\mathfrak{g}^{(0)}=\lambda^{-1}\left(\tau_{1}+\tau_{2}\right)^{-1}, \mathfrak{G}_{-1}=K \mathfrak{g} \mathcal{G}_{-1}+\mathfrak{g} \mathcal{H}_{-1}$.

Proof It follows from formulae (7.9), (7.23) and (9.4) and formulae (4.1), (4.5) for the vector field $V(X)$ and the function $\mathfrak{a}(X)$ that $K, p_{1}$ and $\mathfrak{g}$ are algebraic functions of $\eta, \psi, X^{\prime}$ and its derivatives up to the third order. Moreover they are analytic at the point $\eta=\psi=0$ and $X^{\prime}=\mathbb{T}^{-1}$ and $p_{1}$ vanishes at this point. On the other hand, the matrix $\mathbb{G}_{1}$ and symbol $\check{G}_{0}$, defined by (8.12)-(8.16) are rational functions of $\nabla \eta$ and $X^{\prime}$ which are analytic at the point $X^{\prime}=\mathbb{T}^{-1}, \nabla \eta=0$, and $\check{G}_{0}$ vanishes at this point. From this and formulae (9.6), (9.7), (9.9)-(9.11) we conclude that estimates (9.25)-(9.26) for $A, B, Q_{0}$ and estimate (9.27) for $\mathfrak{g}$ are a straightforward consequence of the inequalities (9.16). Now we estimate the symbol $W$. It follows from (H.2) that for every $u \in C^{s}\left(\mathbb{R}^{2} /(2 \pi \mathbb{Z})^{2}\right)$, and $s \geq 3$

$$
\begin{aligned}
& \left\|\mathcal{D}^{-1} u\right\|_{\mathcal{C}^{s-3}\left(\mathbb{R}^{2} /(2 \pi \mathbb{Z})^{2}\right)} \leq c(s)\left\|\mathcal{D}^{-1} u\right\|_{s-4 / 3} \leq \\
& \leq c(s)\|u\|_{s} \leq c(s)\|u\|_{\mathcal{C}^{s}\left(\mathbb{R}^{2} /(2 \pi \mathbb{Z})^{2}\right)},
\end{aligned}
$$

which leads to inequality (9.26) for the symbol $W$. Next note that by virtue (9.12) - (9.14) the symbols $B_{i}$ and $E$ are rational functions of symbols $W, A$ and $B$ and their derivatives up to second order. These functions are analytic in the domain $|W|<1$. Hence estimates (9.26) for $B_{i}$ and $E$ follow from estimates (9.26) for $A, W$ and $B$. Finally, estimate (9.27) is a consequence of estimate (8.18) for the norm of $\mathcal{G}_{-1}$ and of the form of $\mathcal{H}_{-1}$ (7.30) with $\mathbf{p}_{2}$ and $p_{3}$ defined by $(7.23)$, and $\boldsymbol{\Xi}_{j}(u, 0)$ coming from $\mathbf{T}\left[\nabla u,-\frac{1}{H \mathfrak{a}} \mathcal{D} u\right]$ in (7.10). 
Canonical form. The second preliminary step is the reduction of operator (9.1) to the canonical form with the operators $\mathfrak{A}$ and $\mathfrak{B}$ in the principal part. The corresponding result is given by the following proposition.

Proposition 1 Under the assumptions of Theorem 8 , there are $\epsilon_{0}$ and $c>$ 0 , depending only on $r$ and on the constant $c_{0}$ in conditions (H.1)-(H.2), such that for every $\epsilon \in\left(0, \epsilon_{0}\right]$, there are linear operators $\mathfrak{S}_{-1}: H_{o}^{s} \rightarrow H_{o}^{s}$, $\mathfrak{F}_{-1}: H_{o}^{s} \rightarrow H_{o}^{s+1}$ such that

$$
\mathfrak{S}_{-1}\left\{\mathfrak{g}\left(K \overline{\mathcal{G}}+\frac{1}{H^{2} \widetilde{\mathfrak{a}}} \mathcal{D}^{2}+p_{1} \mathcal{D}+\mathcal{H}_{-1}\right)\right\}=\mathfrak{L}+\mathfrak{A} \mathcal{D}+\mathfrak{B}+\mathfrak{F}_{-1}
$$

For any exponents $s \in[0, r-10]$ and any function $u \in H_{o}^{s}$, the operators $\mathfrak{S}_{-1}$ and $\mathfrak{F}_{-1}$ admit the estimates

$$
\left\|u-\mathfrak{S}_{-1}^{ \pm 1} u\right\|_{s} \leq c \epsilon^{1 / 2}\|u\|_{s}, \quad\left\|\mathfrak{F}_{-1} u\right\|_{s+1} \leq c \epsilon^{1 / 2}\|u\|_{s} .
$$

Proof Using the identity $g_{11}+2 \rho g_{12}+\rho^{2} g_{22}=\mathbb{G} \varrho \cdot \varrho$, we can rewrite the expression for the symbol of the operator $\mathcal{G}_{1}$ in the form

$$
G_{1}(Y, \mathbf{k})=-\frac{\sqrt{\mathbb{G} \varrho \cdot \varrho}}{\left|X^{\prime}(Y)\right|}\left(\mathbb{G}_{1} \tilde{\mathbf{k}} \cdot \widetilde{\mathbf{k}}\right)^{1 / 2}
$$

where the elements of the matrix $\mathbb{G}_{1}(Y)$ are given by (9.3). Recalling the formula (7.9) for the coefficient $K$ in Theorem 5 and the identity $\mathbb{G}=X^{\prime *} \mathbb{Q} X^{\prime}$ we obtain $K \sqrt{\mathbb{G} \varrho \cdot \varrho}=H \sqrt{\mathbb{Q} V \cdot V}$, which gives the expression

$$
K \mathcal{G}_{1}=-\left|X^{\prime}\right|^{-1} \sqrt{\mathbb{Q} V \cdot V} H \mathfrak{G}_{1} .
$$

Here $\mathfrak{G}_{1}$ is the pseudodifferential operator with the symbol $J_{1}$ defined by (9.5). It follows from this, formulae (9.4) for $\mathfrak{g}$ and (7.9) for $H$ that the principal terms in the operator (9.1) can be rewritten in the form

$$
\begin{aligned}
K \mathcal{G}_{1} u+\frac{1}{H^{2} \mathfrak{\mathfrak { a }}} \mathcal{D}^{2} u & =-\frac{\sqrt{\widetilde{\mathbb{Q}} \widetilde{V} \cdot \widetilde{V}}}{\left|X^{\prime}\right|} H\left(\mathfrak{G}_{1} u-\frac{\left|X^{\prime}\right|}{H^{3} \widetilde{\mathfrak{a}} \sqrt{\widetilde{\mathbb{Q}} \widetilde{V} \cdot \widetilde{V}}} \mathcal{D}^{2} u\right) \\
& =\mathfrak{g}^{-1}\left(\mathfrak{G}_{1} u+\nu \mathcal{D}^{2} u\right) .
\end{aligned}
$$

Substituting this expression in (9.1) gives

$$
\mathfrak{g}\left(K \overline{\mathcal{G}}+\frac{1}{H^{2} \widetilde{\mathfrak{a}}} \mathcal{D}^{2}+p_{1} \mathcal{D}+\mathcal{H}_{-1}\right)=\nu \mathcal{D}^{2}+p \mathcal{D}+\mathfrak{G}_{1}+\mathfrak{G}_{0}+\mathfrak{G}_{-1},
$$

where

$$
\mathfrak{G}_{0}=K \mathfrak{g} \mathcal{G}_{0} \quad \mathfrak{G}_{-1}=K \mathfrak{g} \mathcal{G}_{-1}+\mathfrak{g} \mathcal{H}_{-1}, \quad p=\mathfrak{g} p_{1} .
$$

Next notice that by virtue of (9.5)-(9.7), the symbol of the operator $\mathfrak{G}_{1}$ has the representation

$$
\begin{array}{r}
J_{1}=\left|\mathbb{A}^{1 / 2} \widetilde{\mathbf{k}}\right|\left(1+2 \mathbb{B}_{12} \xi_{1} \xi_{2}+\mathbb{B}_{11} \xi_{1}^{2}\right)^{1 / 2} \equiv \\
\equiv\left|\mathbb{A}^{1 / 2} \widetilde{\mathbf{k}}\right|\left(1+\mathbb{B}_{12} \xi_{1} \xi_{2}+\frac{1}{2} \mathbb{B}_{11} \xi_{1}^{2}\right)+\left|\mathbb{A}^{1 / 2} \widetilde{\mathbf{k}}\right| Q_{01} \xi_{1}^{2},
\end{array}
$$


where the symbol $Q_{01}$ is given by (9.11). Next note that we have

$$
\xi_{1}^{2}=-\frac{1}{\nu} \frac{L(\mathbf{k})}{\left|\mathbb{A}^{1 / 2} \widetilde{\mathbf{k}}\right|^{2}}+\frac{1}{\nu\left|\mathbb{A}^{1 / 2} \widetilde{\mathbf{k}}\right|^{2}},
$$

which yields

$$
J_{1}=\left|\mathbb{A}^{1 / 2} \widetilde{\mathbf{k}}\right|-i \xi_{2} B_{12} i\left(k_{1}+\rho k_{2}\right)+Q_{0}(Y, \boldsymbol{\xi})-Q_{0}(Y, \boldsymbol{\xi}) \frac{L(\mathbf{k})}{\left|\mathbb{A}^{1 / 2} \widetilde{\mathbf{k}}\right|} .
$$

Recall that $L(\mathbf{k})=\left|\mathbb{A}^{1 / 2} \widetilde{\mathbf{k}}\right|-\nu\left(k_{1}+\rho k_{2}\right)^{2}$ is the symbol of the pseudodifferential operator $\mathfrak{L}$, and $\left|\mathbb{A}^{1 / 2} \widetilde{\mathbf{k}}\right|$ is the symbol of the differential operator $(-\Delta)^{1 / 2}$. It follows from this that

$$
p \mathcal{D}+\mathfrak{G}_{1}=(-\Delta)^{1 / 2}+\mathfrak{A D}+\mathfrak{Q}_{0}-\mathfrak{Q}_{0}(-\Delta)^{-1 / 2} \mathfrak{L}
$$

which leads to the identity

$$
\nu \mathcal{D}^{2} u+p \mathcal{D}+\mathfrak{G}_{1}+\mathfrak{G}_{0}=\left(\mathbf{I}-\mathfrak{Q}_{0}(-\Delta)^{-1 / 2}\right) \mathfrak{L}+\mathfrak{A D}+\mathfrak{Q}_{0}+\mathfrak{G}_{0} .
$$

Note that $\mathfrak{A D}=\mathcal{D A}-\mathfrak{A}_{1}$, where $\mathfrak{A}_{1}$ is a zero order operator with the symbol $\mathcal{D} A(Y, \boldsymbol{\xi})$. Thus we get

$$
\mathfrak{A D}=\left(\mathbf{I}-\mathfrak{Q}_{0}(-\Delta)^{-1 / 2}\right) \mathfrak{A} \mathcal{D}+\mathfrak{Q}_{0}(-\Delta)^{-1 / 2} \mathcal{D} \mathfrak{A}-\mathfrak{Q}_{0}(-\Delta)^{-1 / 2} \mathfrak{A}_{1} .
$$

The symbol of the operator $(-\Delta)^{-1 / 2} \mathcal{D}$ is simply $i \xi_{1}$, and the operator $\mathfrak{Q}_{02}:=$ $\mathfrak{Q}_{0}(-\Delta)^{-1 / 2} \mathcal{D}$ is a zero order pseudo-differential operator with the symbol $Q_{02}=i Q_{0}(Y, \boldsymbol{\xi}) \xi_{1}$. It follows from this and identity (8.20) in Lemma 9 that the operator $\mathfrak{Q}_{0}(-\Delta)^{-1 / 2} \mathcal{D} \mathfrak{A}$ has the representation

$$
\mathfrak{Q}_{0}(-\Delta)^{-1 / 2} \mathcal{D} \mathfrak{A} \equiv \mathfrak{Q}_{02} \mathfrak{A}=\left(\mathfrak{Q}_{02} \mathfrak{A}\right)_{0}+\mathfrak{D}_{1}^{\left(\mathfrak{Q}_{02} \mathfrak{A}\right)}
$$

where the operator $\left(\mathfrak{Q}_{02} \mathfrak{A}\right)_{0}$ has the symbol $i \xi_{1} Q_{0} A$. Combining these results we arrive at the identity

$$
\mathfrak{A D}=\left(\mathbf{I}-\mathfrak{Q}_{0}(-\Delta)^{-1 / 2}\right)\left(\mathfrak{A D}+\left(\mathfrak{Q}_{02} \mathfrak{A}\right)_{0}\right)+\mathfrak{F}_{-1,0}
$$

where the linear operator $\mathfrak{F}_{-1,0}$ is given by

$$
\left.\mathfrak{F}_{-1,0}=\mathfrak{Q}_{0}(-\Delta)^{-1 / 2}\right)\left(\mathfrak{Q}_{02} \mathfrak{A}\right)_{0}+\mathfrak{D}_{1}^{\left(\mathfrak{Q}_{02} \mathfrak{A}\right)}-\mathfrak{Q}_{0}(-\Delta)^{-1 / 2} \mathfrak{A}_{1} .
$$

Thus we get

$$
\mathfrak{A D}+\mathfrak{Q}_{0}+\mathfrak{G}_{0}=\left(\mathbf{I}-\mathfrak{Q}_{0}(-\Delta)^{-1 / 2}\right)\left(\mathfrak{A D}+\left(\mathfrak{Q}_{02} \mathfrak{A}\right)_{0}+\mathfrak{Q}_{0}+\mathfrak{G}_{0}\right)+\mathfrak{F}_{-1,1}
$$

where

$$
\mathfrak{F}_{-1,1}=\mathfrak{Q}_{0}(-\Delta)^{-1 / 2}\left(\mathfrak{Q}_{0}+\mathfrak{G}_{0}+\left(\mathfrak{Q}_{02} \mathfrak{A}\right)_{0}-\mathfrak{A}_{1}\right)+\mathfrak{D}_{1}^{\left(\mathfrak{Q}_{02} \mathfrak{A}\right)} .
$$

Since $\mathfrak{B}=\left(\mathfrak{Q}_{02} \mathfrak{A}\right)_{0}+\mathfrak{Q}_{0}+\mathfrak{G}_{0}$, we conclude from this that identity (9.29) is fulfilled if we take

$$
\mathfrak{S}_{-1}=\left(\mathbf{I}-\mathfrak{Q}_{0}(-\Delta)^{-1 / 2}\right)^{-1} \text { and } \mathfrak{F}_{-1}=\mathfrak{S}_{-1}\left(\mathfrak{F}_{-1,1}+\mathfrak{G}_{-1}\right) .
$$


It remains to prove estimate $(9.30)$. We begin with the observation that, by virtue of inequality (9.26) for $Q_{0}$ and estimate (8.19) in Lemma 9, we have $\left\|\mathfrak{Q}_{0} u\right\|_{s} \leq c \epsilon^{1 / 2}\|u\|_{s}$ for all $u \in H_{o}^{s}, s \in[0, r-6]$. Hence for all such exponents $s$, the operator $\mathfrak{U} \equiv \mathfrak{S}_{-1}$ meets all requirements of Lemma 11 which gives the desired estimates for $\mathfrak{S}_{-1}$. Next, it follows from the equalities

$$
J_{0}=K \mathfrak{g} \check{G}_{0}, \quad Q_{02}=i \xi_{1} Q_{0}, \quad A_{1}=\mathcal{D} A
$$

and estimates (9.26), (8.17) that

$$
\left|Q_{0}+J_{0}+Q_{02} A\right|_{r-4}+\left|A_{1}\right|_{r-4} \leq c \epsilon^{1 / 2} .
$$

From this and Lemma 9 we conclude that for all $u \in H_{o}^{s}, s \in[0, r-7]$,

$$
\left\|\left(\mathfrak{Q}_{0}+\mathfrak{G}_{0}+\left(\mathfrak{Q}_{02} \mathfrak{A}\right)_{0}\right) u\right\|_{s}+\left\|\mathfrak{A}_{1} u\right\|_{s} \leq c \epsilon^{1 / 2}\|u\|_{s} .
$$

Obviously we have

$$
\left\|(-\Delta)^{1 / 2} u\right\|_{s+1} \leq c(s)\|u\|_{s} \text { for all } s \geq 0 .
$$

Using inequalities (9.26) for $Q_{0}$ and estimate (8.19) in Lemma 9, we obtain from this that for all $u \in H_{o}^{s}, s \in[0, r-8]$,

$$
\left\|\mathfrak{Q}_{0}(-\Delta)^{1 / 2} u\right\|_{s+1} \leq c \epsilon^{1 / 2}\|u\|_{s} .
$$

In its turn, inequalities (9.26) for $Q_{0}$ and $A$ along with estimate (8.22) in Lemma 9 for the remainder $\mathfrak{D}_{1}$ imply for all $u \in H_{o}^{s}, s \in[0, r-10]$,

$$
\left\|\mathfrak{D}_{1}^{\left(\mathfrak{Q}_{02} \mathfrak{A}\right)} u\right\|_{s+1} \leq c \epsilon^{1 / 2}\|u\|_{s} .
$$

Combining this estimate with (9.34) and (9.35) we obtain that the inequality

$$
\left\|\mathfrak{F}_{-1,1} u\right\|_{s+1} \leq c \epsilon^{1 / 2}\|u\|_{s}
$$

holds true for all $u \in H_{o}^{s}, s \in[0, r-10]$. This result along with the estimate for $\mathfrak{S}_{-1}$, gives estimates $(9.30)$ for $\mathfrak{F}_{-1}$ and the proposition follows.

\subsection{Descent method}

We split the rest of the proof into three propositions which correspond to three steps in the so called descent method [16]. We can make the first step of the descend method as in [16] in order to eliminate the first order term $\mathfrak{A D}$ in $\mathfrak{L}+\mathfrak{A D}+\mathfrak{B}+\mathfrak{F}_{-1}$. The corresponding result is given by the following

first step Proposition 2 Under the assumptions of Theorem 8, there exists $\epsilon_{0}$ depending only on $r$, and the constant $c_{0}$ in Condition $(\boldsymbol{H} . \mathbf{1})$ with the following properties. For any $\epsilon \in\left(0, \epsilon_{0}\right)$, there are bounded operators $\mathfrak{S}_{0}, \mathfrak{T}_{0}: H^{s} \mapsto H^{s}$, $\mathfrak{F}_{0}: H^{s} \mapsto H^{s+1}$ so that

$$
\mathfrak{S}_{0}\left(\mathfrak{L}+\mathfrak{A D}+\mathfrak{B}+\mathfrak{F}_{-1}\right) \mathfrak{T}_{0}=\mathfrak{L}+\mathfrak{B}_{0}+\mathfrak{F}_{0},
$$

where $\mathfrak{B}_{0}$ is a zero-order pseudodifferential operator with the symbol given by formula (9.12). Moreover, for all $u \in H_{o}^{s}, s \in[0, r-15]$,

$$
\begin{gathered}
\left\|\mathfrak{F}_{0} u\right\|_{s+1} \leq c \epsilon^{1 / 2}\|u\|_{s}, \\
\left\|\left(\mathfrak{S}_{0}^{ \pm 1}-\mathbf{I}\right) u\right\|_{s}+\left\|\left(\mathfrak{T}_{0}^{ \pm 1}-\mathbf{I}\right) u\right\|_{s} \leq c \epsilon^{1 / 2}\|u\|_{s} .
\end{gathered}
$$


Proof Let $\tilde{W}(y)$ be an arbitrary smooth periodic function, and $\mathfrak{W}$ be a pseudodifferential operator associated with the symbol $\operatorname{Re} \tilde{W}(Y)+i \xi_{2} \operatorname{Im} \tilde{W}(Y)$. It is easy to see that

$$
\begin{aligned}
& (\mathfrak{L}+\mathfrak{A D}+\mathfrak{B}) \mathfrak{W}=\mathfrak{W} \mathfrak{L}+\left(2 \nu \mathfrak{W}_{1}+\mathfrak{A} \mathfrak{W}\right) \mathcal{D}+ \\
& \nu \mathfrak{W}_{11}+\mathfrak{A W}_{1}+\mathfrak{B W}+\left[(-\Delta)^{1 / 2}, \mathfrak{W}\right],
\end{aligned}
$$

where the zero order differential operators $\mathfrak{W}_{1}, \mathfrak{W}_{11}$ have the symbols

$$
W_{1}=\mathcal{D} W \equiv\left(\partial_{y_{1}}+\rho \partial_{y_{2}}\right) W, \quad W_{11}=\mathcal{D}^{2} W .
$$

It follows from representation (8.20) in Lemma 9 that

$$
\begin{aligned}
\mathfrak{A W} & =(\mathfrak{A W})_{0}+(\mathfrak{A W})_{1}+\mathfrak{D}_{2}^{(\mathfrak{A} \mathfrak{W})}, \\
\mathfrak{A W}_{1} & =\left(\mathfrak{A} \mathfrak{W}_{1}\right)_{0}+\mathfrak{D}_{1}^{\left.(\mathfrak{A} \mathfrak{W})_{1}\right)}, \quad \mathfrak{B W}=(\mathfrak{B W})_{0}+\mathfrak{D}_{1}^{(\mathfrak{B W})}
\end{aligned}
$$

where the zero order operators $(\mathfrak{A W})_{0},\left(\mathfrak{A} \mathfrak{W}_{1}\right)_{0}$, and $(\mathfrak{B W})_{0}$ have the symbols

$$
W(Y, \boldsymbol{\xi}) A(Y, \boldsymbol{\xi}), \quad \mathcal{D} W(Y, \boldsymbol{\xi}) A(Y, \boldsymbol{\xi}) \text { and } W(Y, \boldsymbol{\xi}) B(Y, \boldsymbol{\xi}) .
$$

Finally, using the representations (8.21) for the symbol of the operator $(\mathfrak{A W})_{1}$ and (8.23) for the commutator we arrive at the identity

$$
(\mathfrak{L}+\mathfrak{A D}+\mathfrak{B}) \mathfrak{W}=\mathfrak{W} \mathfrak{L}+2 \nu \mathfrak{W}_{1} \mathcal{D}+(\mathfrak{A} \mathfrak{W})_{0} \mathcal{D}+\mathfrak{B}_{1}+\mathfrak{F}_{0,1},
$$

where $\mathfrak{B}_{1}$ is a zero order operator with the symbol

$$
\begin{aligned}
B_{1}=\nu \mathcal{D}^{2} W+A \mathcal{D} W & +B W+ \\
& +\nabla_{k} A \cdot \nabla_{Y} W\left(k_{1}+\rho k_{2}\right)-i \nabla_{Y} W \cdot \nabla_{k}(\mathbb{A} \widetilde{\mathbf{k}} \cdot \widetilde{\mathbf{k}})^{1 / 2},
\end{aligned}
$$

and the regularizing operator

$$
\mathfrak{F}_{0,1}=\mathfrak{D}_{2}^{(\mathfrak{A}, \mathfrak{W})} \mathcal{D}+\mathfrak{D}_{2}^{\left[(-\Delta)^{1 / 2}, \mathfrak{W}\right]}+\mathfrak{D}_{1}^{\left(\mathfrak{A} \mathfrak{W}_{1}\right)}+\mathfrak{D}_{1}^{(\mathfrak{B} \mathfrak{W})} .
$$

Simple calculations show that $B_{1}$ depend on $\boldsymbol{\xi}$ and $Y$ and satisfies equality (9.13). Next we rearrange the expression for $(\mathfrak{A} \mathfrak{W})_{0} \mathcal{D}$. To this end note that the symbol of this operator is equal to $i A W\left(k_{1}+\rho k_{2}\right)$. We have

$$
\begin{aligned}
& A W=\operatorname{Re} \tilde{A} \operatorname{Re} \tilde{W}-\xi_{2}^{2} \operatorname{Im} \tilde{A} \operatorname{Im} \tilde{W}+i \xi_{2} \operatorname{Im}(\tilde{W} \tilde{A})= \\
& \operatorname{Re}(\tilde{W} \tilde{A})+i \xi_{2} \operatorname{Im}(\tilde{W} \tilde{A})+\operatorname{Im} \tilde{A} \operatorname{Im} \tilde{W}\left(1-\xi_{2}^{2}\right) .
\end{aligned}
$$

Combining this result with the identity

$$
\left(1-\xi_{2}^{2}\right)=(\mathbb{A} \widetilde{\mathbf{k}} \cdot \widetilde{\mathbf{k}})^{-1}\left(2 A_{12}\left(k_{1}+\rho k_{2}\right) k_{2}+A_{11}\left(k_{1}+\rho k_{2}\right)^{2}\right)
$$

gives

$$
\begin{aligned}
i A W\left(k_{1}+\rho k_{2}\right) & =i\left(\operatorname{Re} \tilde{N}+i \xi_{2} \operatorname{Im} \tilde{N}\right)\left(k_{1}+\rho k_{2}\right)+ \\
& +i(\operatorname{Im} \tilde{A} \operatorname{Im} \tilde{W})(\mathbb{A} \widetilde{\mathbf{k}} \cdot \widetilde{\mathbf{k}})^{-1 / 2}\left(k_{1}+\rho k_{2}\right)^{2}\left(2 A_{12} \xi_{2}+A_{11} \xi_{1}\right),
\end{aligned}
$$


where $\tilde{N}=\tilde{A} \tilde{W}$. Using the identity

$$
(\mathbb{A} \widetilde{\mathbf{k}} \cdot \widetilde{\mathbf{k}})^{-1 / 2}\left(k_{1}+\rho k_{2}\right)^{2}=-\nu^{-1} L(\mathbf{k})(\mathbb{A} \widetilde{\mathbf{k}} \cdot \widetilde{\mathbf{k}})^{-1 / 2}+\nu^{-1},
$$

we obtain

$$
i A W\left(k_{1}+\rho k_{2}\right)=i N\left(k_{1}+\rho k_{2}\right)+E-E(\mathbb{A} \tilde{\mathbf{k}} \cdot \widetilde{\mathbf{k}})^{-1 / 2} L(\mathbf{k}) .
$$

Here $N=\operatorname{Re} \tilde{N}(Y)+i \xi_{2} \operatorname{Im} \tilde{N}(Y)$ is the symbol of the elementary operator $\mathfrak{N}$ associated with the function $\tilde{N}$ and the symbol $E$ is determined by (9.14). Thus we get

$$
(\mathfrak{A W})_{0} \mathcal{D}=\mathfrak{N D}+\mathfrak{E}-\mathfrak{E}(-\Delta)^{-1 / 2} \mathfrak{L},
$$

where $\mathfrak{E}$ is a zero-order differential operator with the symbol $E$. Substituting this expression into (9.39) leads to

$$
\begin{aligned}
&(\mathfrak{L}+\mathfrak{A D}+\mathfrak{B}) \mathfrak{W}=\left(\mathfrak{W}-\mathfrak{E}(-\Delta)^{-1 / 2}\right) \mathfrak{L}+ \\
&\left(2 \nu \mathfrak{W}_{1}+\mathfrak{N}\right) \mathcal{D}+\left(\mathfrak{B}_{1}+\mathfrak{E}\right)+\mathfrak{F}_{0,1},
\end{aligned}
$$

which gives the important identity

$$
\begin{aligned}
(\mathfrak{L}+\mathfrak{A D}+\mathfrak{B})(\mathbf{I}+\mathfrak{W}) & =\left(\mathbf{I}+\mathfrak{W}-\mathfrak{E}(-\Delta)^{-1 / 2}\right) \mathfrak{L}+ \\
& +\left(2 \nu \mathfrak{W}_{1}+\mathfrak{N}+\mathfrak{A}\right) \mathcal{D}+\mathfrak{B}_{0,1}+\mathfrak{F}_{0,1},
\end{aligned}
$$

where $\mathfrak{B}_{0,1}$ is a zero order operator with the symbol $B_{1}+B+E$.

The next step is the key point of our method. We specify the operator $\mathfrak{W}$ so that $2 \nu \mathfrak{W}_{1}+\mathfrak{N}+\mathfrak{A}=0$. To this end, notice that this operator is an elementary operator associated with the function $2 \nu \mathcal{D} \tilde{W}+\tilde{A} \tilde{W}+\tilde{A}$ which leads to the equation

$$
2 \nu \mathcal{D} \tilde{W}(Y)+\tilde{W}(Y) \tilde{A}(Y)+\tilde{A}(Y)=0 .
$$

Since the function $\tilde{A}(\cdot, \boldsymbol{\xi})$ has a zero mean value over a period, we can take a solution to this equation in the form (9.8). By choosing such $\mathfrak{W}$, the identity (9.40) becomes

$$
\begin{aligned}
\left(\mathfrak{L}+\mathfrak{A D}+\mathfrak{B}+\mathfrak{F}_{-1}\right)(\mathbf{I}+\mathfrak{W}) & =\left(\mathbf{I}+\mathfrak{W}-\mathfrak{E}(-\Delta)^{-1 / 2}\right) \mathfrak{L}+ \\
& +\mathfrak{B}_{0,1}+\mathfrak{F}_{0,1}+\mathfrak{F}_{-1}(\mathbf{I}+\mathfrak{W}) .
\end{aligned}
$$

Next we introduce the zero order operator $\mathfrak{B}_{0}$ with the symbol given by (9.12). Notice that

$$
(\mathbf{I}+\mathfrak{W}) \mathfrak{B}_{0}=\left((\mathbf{I}+\mathfrak{W}) \mathfrak{B}_{0}\right)_{0}+\mathfrak{D}_{1}^{\left((\mathbf{I}+\mathfrak{W}) \mathfrak{B}_{0}\right)} .
$$

By virtue of (8.20) and (9.12), we have $\left((\mathbf{I}+\mathfrak{W}) \mathfrak{B}_{0}\right)_{0}=\mathfrak{B}_{0,1}$ which leads to the identity

$$
\begin{aligned}
& \mathfrak{B}_{0,1}=(\mathbf{I}+\mathfrak{W}) \mathfrak{B}_{0}-\mathfrak{D}_{1}^{\left((\mathbf{I}+\mathfrak{W}) \mathfrak{B}_{0}\right)}= \\
& \quad\left(\mathbf{I}+\mathfrak{W}-\mathfrak{E}(-\Delta)^{-1 / 2}\right) \mathfrak{B}_{0}+\mathfrak{E}(-\Delta)^{-1 / 2} \mathfrak{B}_{0}-\mathfrak{D}_{1}^{\left((\mathbf{I}+\mathfrak{W}) \mathfrak{B}_{0}\right)}
\end{aligned}
$$


Hence we can rewrite identity (9.41) in the form (9.36) with

$$
\mathfrak{S}_{0}=\left(\mathbf{I}+\mathfrak{W}-\mathfrak{E}(-\Delta)^{-1 / 2}\right)^{-1}, \quad \mathfrak{T}_{0}=\mathbf{I}+\mathfrak{W}, \quad \mathfrak{F}_{0}=\mathfrak{S}_{0} \mathfrak{F}_{0,2}, \quad(9.42) \quad 15.23
$$

where

$$
\mathfrak{F}_{0,2}=\mathfrak{F}_{0,1}+\mathfrak{F}_{-1}(\mathbf{I}+\mathfrak{W})+\mathfrak{E}(-\Delta)^{-1 / 2} \mathfrak{B}_{0}-\mathfrak{D}_{1}^{\left((\mathbf{I}+\mathfrak{W}) \mathfrak{B}_{0}\right)} .
$$

It remains to prove that the operators $\mathfrak{F}_{0}, \mathfrak{S}_{0}$, and $\mathfrak{T}_{0}$ meet all requirements of Proposition 2. Applying inequality (8.19) to the operator $\mathfrak{W}$ and using inequalities (9.26) for the symbol $W$ we obtain for all $u \in H_{o}^{s}, s \in[r-11]$,

$$
\|\mathfrak{W} u\|_{s} \leq c \epsilon^{1 / 2}\|u\|_{s} .
$$

Let us estimate the remainder $\mathfrak{F}_{0}$. Since $r-8 \geq 7$, it follows from estimate (8.22), with $l$ replaced by $r-6$, and inequalities (9.26) that for all $u \in H_{o}^{s}$, $s \in[0, r-13]$

$$
\left\|\mathfrak{D}_{2}^{(\mathfrak{A} \mathfrak{W})} u\right\|_{s+2} \leq c \epsilon^{1 / 2}\|u\|_{s} .
$$

Since the operator $\mathcal{D}: H^{s} \rightarrow H^{s-1}$ is bounded, we obtain for all $u \in H_{o}^{s}$, $s \in[1, r-12]$,

$$
\left\|\mathfrak{D}_{2}^{(\mathfrak{A} \mathfrak{W})} \mathcal{D} u\right\|_{s+1} \leq c \epsilon^{1 / 2}\|u\|_{s}
$$

It also follows from (8.22) and inequalities (9.26) that for all $u \in H_{o}^{s}, s \in$ $[0, r-14]$,

$$
\left\|\mathfrak{D}_{1}^{\left(\mathfrak{A} \mathfrak{W}_{1}\right)} u\right\|_{s+1}+\left\|\mathfrak{D}_{1}^{(\mathfrak{B} \mathfrak{W})} u\right\|_{s+1} \leq c \epsilon^{1 / 2}\|u\|_{s} .
$$

Inequalities (9.26) along with estimate (8.24), imply for all $u \in H_{o}^{s}, s \in$ $[0, r-13]$,

$$
\left\|\mathfrak{D}_{2}^{\left[(-\Delta)^{1 / 2}, \mathfrak{W}\right)} u\right\|_{s+1} \leq c \epsilon^{1 / 2}\|u\|_{s} .
$$

Combining these results with formula for $\mathfrak{F}_{0,1}$ we obtain that for all $u \in H_{o}^{s}$, $s \in[1, r-14]$,

$$
\left\|\mathfrak{F}_{0,1} u\right\|_{s+1} \leq c \epsilon^{1 / 2}\|u\|_{s} .
$$

Next combining inequalities (9.44) and (9.30) we obtain for all $u \in H_{o}^{s}$, $s \in[0, r-11]$,

$$
\left\|\mathfrak{F}_{-1}(\mathbf{I}+\mathfrak{W}) u\right\|_{s+1} \leq c \epsilon^{1 / 2}\|u\|_{s} .
$$

Applying estimates (9.26) and (8.19) to the operator $\mathfrak{E}$, we conclude that the inequality

$$
\|\mathfrak{E} u\|_{s} \leq c \epsilon^{1 / 2}\|u\|_{s}
$$

holds true for all $u \in H_{o}^{s}, s \in[0, r-11]$. In particular, we have for all $u \in H_{o}^{s}$, $s \in[0, r-12]$,

$$
\left\|\mathfrak{E}(-\Delta)^{-1 / 2} u\right\|_{s+1} \leq c \epsilon^{1 / 2}\|u\|_{s} .
$$

Inequalities (9.26) for the symbols $W$ and $B_{0}$ along with estimate (8.22) with $\mathfrak{A}$ and $\mathfrak{W}$ replaced by $\mathbf{I}+\mathfrak{W}$ and $\mathfrak{B}_{0}$ imply that for all $u \in H_{o}^{s}, s \in[1, r-15]$

$$
\left\|\mathfrak{D}_{1}^{\left((\mathbf{I}+\mathfrak{W}) \mathfrak{B}_{0}\right)} u\right\|_{s+1} \leq c \epsilon^{1 / 2}\|u\|_{s}
$$


Combining estimates (9.45)- (9.49) we finally obtain for all $u \in H_{o}^{s}, s \in$ $[1, r-15]$,

$$
\left\|\mathfrak{F}_{0,2} u\right\|_{s+1} \leq c \epsilon^{1 / 2}\|u\|_{s}
$$

Now our task is to derive the estimates (9.38) for the norms of the operators $\mathfrak{S}_{0}$ and $\mathfrak{T}_{0}$. It follows from (9.44) and (9.48) that for all $s \in[0, r-12]$, the operators $\mathfrak{U}=\mathfrak{W}$ and $\mathfrak{U}=\mathfrak{W}-\mathfrak{E}(-\Delta)^{-1 / 2}$ meet all requirements of Lemma 11. Therefore there exists $\epsilon_{0}$, depending only on $s$ and on the constant $c_{0}$ in conditions $\left(\right.$ H.1)-(H.2), such that for all $\epsilon \in\left[0, \epsilon_{0}\right)$ the operators $\mathfrak{S}_{0}$ and $\mathfrak{T}_{0}$ determined by formulae (9.42) have bounded inverses, and satisfy inequalities (9.38). It remains to note that since $\mathfrak{F}_{0}=\mathfrak{S}_{0} \mathfrak{F}_{0,2}$, estimate $(9.37)$ for the operator $\mathfrak{F}_{0}$ follows from inequality $(9.50)$.

Second and third steps. The next steps in the descent method require again the inversion of the differential operator $\mathcal{D}$. The operator $\mathcal{D}^{-1}$ stands explicitly in the new form of the operator, contrary to the result provided at the end of the first step. The obstacle now is that the inverse $\mathcal{D}^{-1}$ is unbounded in any Sobolev space of periodic functions. This is the principal distinction in the descent method, between the asymmetric and the symmetric water wave problem. We cope with this difficulty by using the projector $\Pi$ defined by equalities (8.26). In particular, Lemma 10 plays the key role in the proof. The following proposition represents the second step in the descent method.

second step Proposition 3 Assume that all assumptions of Theorem 8 are satisfied and operators $\mathfrak{B}_{0}, \mathfrak{F}_{0}$ meet all requirements of Proposition 2. Then there exists $\epsilon_{0}$, depending only on exponent $s$ and on the constant $c_{0}$ in conditions $(\boldsymbol{H} . \mathbf{1})$ $(\boldsymbol{H} .2)$, with the following properties. For any $\epsilon \in\left(0, \epsilon_{0}\right)$, there are bounded operators $\mathfrak{S}_{2}, \mathfrak{T}_{2}: H^{s} \mapsto H^{s}, \mathfrak{F}_{2}: H^{s} \mapsto H^{s+1}$ and a zero-order pseudodifferential operator $\mathfrak{B}_{2}$ so that

$$
\mathfrak{S}_{2}\left(\mathfrak{L}+\mathfrak{B}_{0}+\mathfrak{F}_{0}\right) \mathfrak{T}_{2}=\Pi\left(\mathfrak{L}+\mathfrak{V}+\mathfrak{B}_{2} \mathcal{D}^{-1}+\mathfrak{F}_{2}\right) \Pi+(\mathbf{I}-\Pi)\left(\mathfrak{L}+\mathfrak{P}_{2}\right) .
$$

Here $\mathfrak{V}$ is a zero order pseudodifferential operator with the symbol determined by (9.15). The symbol $B_{2}(Y, \boldsymbol{\xi})$ of the operator $\mathfrak{B}_{2}$ satisfies the conditions

$$
\left|B_{2}\right|_{r-13} \leq c \epsilon^{1 / 2}, \quad \int_{\mathbb{R}^{2} /(2 \pi \mathbb{Z})^{2}} B_{2}(Y, \boldsymbol{\xi}) d Y=0 .
$$

For all $u \in H_{o}^{s}, s \in[0, r-37 / 2]$, operators $\mathfrak{F}_{2}, \mathfrak{S}_{2}, \mathfrak{T}_{2}$, and $\mathfrak{P}_{2}$ satisfy the inequalities

$$
\begin{gathered}
\left\|\mathfrak{F}_{2} u\right\|_{s+1} \leq c \epsilon^{1 / 2}\|u\|_{s}, \quad\left\|\mathfrak{P}_{2} u\right\|_{s} \leq c \epsilon^{1 / 2}\|u\|_{s}, \\
\left\|\left(\mathfrak{S}_{2}^{ \pm 1}-\mathbf{I}\right) u\right\|_{s}+\left\|\left(\mathfrak{T}_{2}^{ \pm 1}-\mathbf{I}\right) u\right\|_{s} \leq c \epsilon^{1 / 2}\|u\|_{s},
\end{gathered}
$$

where $c$ depends only on $s$, and $c_{0}$. 
Proof We split the proof into two parts. First we give the formal derivation of identity (9.51) and next justify our construction by proving inequalities (9.53)-(9.54). We begin with the reduction of the operator $\mathfrak{L}+\mathfrak{B}_{0}+\mathfrak{F}_{0}$ to a triangular form. It is easy to check that

$$
\mathfrak{L}^{-1}(\mathbf{I}-\Pi)\left(\mathfrak{L}+\mathfrak{B}_{0}+\mathfrak{F}_{0}\right) \equiv(\mathbf{I}+\mathfrak{K}(\mathbf{I}-\Pi))(\mathbf{I}-\Pi)+\mathfrak{K} \Pi,
$$

where $\mathfrak{K}=\mathfrak{L}^{-1}(\mathbf{I}-\Pi)\left(\mathfrak{B}_{0}+\mathfrak{F}_{0}\right)$. Let us introduce the linear operator

$$
\mathfrak{Q}_{2}=\left(\mathfrak{B}_{0}+\mathfrak{F}_{0}\right)(\mathbf{I}+\mathfrak{K}(\mathbf{I}-\Pi))^{-1} \mathfrak{L}^{-1}(\mathbf{I}-\Pi) .
$$

It follows from (9.55) that

$$
\mathfrak{Q}_{2}\left(\mathfrak{L}+\mathfrak{B}_{0}+\mathfrak{F}_{0}\right) \equiv\left(\mathfrak{B}_{0}+\mathfrak{F}_{0}\right)(\mathbf{I}-\Pi)+\left(\mathfrak{B}_{0}+\mathfrak{F}_{0}\right)(\mathbf{I}+\mathfrak{K}(\mathbf{I}-\Pi))^{-1} \mathfrak{K} \Pi .
$$

From this we obtain

$$
\Pi\left(\mathbf{I}-\mathfrak{Q}_{2}\right)\left(\mathfrak{L}+\mathfrak{B}_{0}+\mathfrak{F}_{0}\right)=\Pi\left(\mathfrak{L}+\mathfrak{B}_{0}+\mathfrak{F}_{2,0}\right) \Pi,
$$

where

$$
\mathfrak{F}_{2,0}=\mathfrak{F}_{0}-\left(\mathfrak{B}_{0}+\mathfrak{F}_{0}\right)(\mathbf{I}+\mathfrak{K}(\mathbf{I}-\Pi))^{-1} \mathfrak{K} .
$$

This identity also can be rewritten in the equivalent form

$$
\begin{aligned}
& \left(\mathbf{I}-\Pi \mathfrak{Q}_{2}\right)\left(\mathfrak{L}+\mathfrak{B}_{0}+\mathfrak{F}_{0}\right)= \\
& =\Pi\left(\mathfrak{L}+\mathfrak{B}_{0}+\mathfrak{F}_{2,0}\right) \Pi+(\mathbf{I}-\Pi)\left(\mathfrak{L}+\mathfrak{B}_{0}+\mathfrak{F}_{0}\right),
\end{aligned}
$$

which can be regarded as the triangular form of the operator $\mathfrak{L}+\mathfrak{B}_{0}+\mathfrak{F}_{0}$. Now our study should be focused on the first term in the right hand side of $(9.56)$. Choose an arbitrary zero order operator $\mathfrak{H}$ with a smooth symbol $H(Y, \boldsymbol{\xi})$. The only restriction is that this operator should protect the oddness property. Since $\mathfrak{L}$ and $\mathcal{D}^{-1}$ commute with $\Pi$, we have

$\Pi \mathfrak{L} \Pi \mathfrak{H} \mathcal{D}^{-1} \Pi=\Pi \mathfrak{H D}^{-1} \mathfrak{L} \Pi+2 \nu \Pi \mathfrak{H}_{1} \Pi+\nu \Pi \mathfrak{H}_{11} \mathcal{D}^{-1} \Pi+\Pi\left[(-\Delta)^{1 / 2}, \mathfrak{H}\right] \mathcal{D}^{-1} \Pi$

which leads to the identity

$$
\begin{aligned}
& \Pi\left(\mathfrak{L}+\mathfrak{B}_{0}+\mathfrak{F}_{2,0}\right) \Pi \mathfrak{H} \mathcal{D}^{-1} \Pi=\Pi \mathfrak{H} \mathcal{D}^{-1} \mathfrak{L} \Pi+2 \nu \Pi \mathfrak{H}_{1} \Pi+ \\
& +\Pi\left(\mathfrak{B}_{0} \Pi \mathfrak{H}+\nu \mathfrak{H}_{11}+\left[(-\Delta)^{1 / 2}, \mathfrak{H}\right]\right) \mathcal{D}^{-1} \Pi+\Pi \mathfrak{F}_{2,0} \Pi \mathfrak{H} \mathcal{D}^{-1} \Pi
\end{aligned}
$$

Here zero order differential operators $\mathfrak{H}_{1}$ and $\mathfrak{H}_{11}$ have the symbols $H_{1}=\mathcal{D} H$ and $H_{11}=\mathcal{D}^{2} H$. Now our task is to rearrange the operators in the right hand side of identity (9.57) through the use of Lemmas 9 and 10 . We begin with the observation that by virtue of Lemma 10 we have

$$
\mathfrak{B}_{0} \Pi \mathfrak{H}=\mathfrak{B}_{0} \mathfrak{H} \Pi+\mathfrak{B}_{0}[\Pi, \mathfrak{H}]=\mathfrak{B}_{0} \mathfrak{H} \Pi+\mathfrak{B}_{0} \mathfrak{Z}+\mathfrak{B}_{0} \mathfrak{Y} \mathfrak{L} .
$$

On the other hand, the product $\mathfrak{B}_{0} \mathfrak{H}$ admits the representation (8.20), with $\mathfrak{A}$ and $\mathfrak{W}$ replaced by $\mathfrak{B}_{0}$ and $\mathfrak{H}$, which gives $\mathfrak{B}_{0} \mathfrak{H}=\left(\mathfrak{B}_{0} \mathfrak{H}\right)_{0}+\mathfrak{D}_{1}^{\left(\mathfrak{B}_{0} \mathfrak{H}\right)}$. We thus get

$$
\begin{aligned}
& \Pi \mathfrak{B}_{0} \Pi \mathfrak{H} \Pi \mathcal{D}^{-1}=\Pi\left(\mathfrak{B}_{0} \mathfrak{H}\right)_{0} \Pi \mathcal{D}^{-1}+ \\
& +\Pi\left[\mathfrak{D}_{1}^{\left(\mathfrak{B}_{0} \mathfrak{H}\right)}+\mathfrak{B}_{0} \mathfrak{Z}\right] \Pi \mathcal{D}^{-1}+\Pi \mathfrak{B}_{0} \mathfrak{Y} \Pi \mathfrak{L D}^{-1} .
\end{aligned}
$$


Next using the representation (8.23) for the commutator we obtain

$$
\begin{aligned}
\Pi\left[(-\Delta)^{-1 / 2}, \mathfrak{H}\right] \Pi \mathcal{D}^{-1} & =\Pi\left[(-\Delta)^{-1 / 2}, \mathfrak{H}\right]_{1} \Pi \mathcal{D}^{-1}+ \\
& +\Pi \mathfrak{D}_{2}^{\left[(-\Delta)^{1 / 2}, \mathfrak{H}\right]} \Pi \mathcal{D}^{-1}
\end{aligned}
$$

Recall that the operators $\left(\mathfrak{B}_{0} \mathfrak{H}\right)_{0}$ and $\left[(-\Delta)^{-1 / 2}, \mathfrak{H}\right]_{1}$ have the symbols $H(Y, \boldsymbol{\xi}) B_{0}(Y, \boldsymbol{\xi})$ and $-i \nabla_{Y} H \cdot \nabla_{k}(\mathbb{A} \widetilde{\mathbf{k}} \cdot \widetilde{\mathbf{k}})^{1 / 2} \equiv-i \boldsymbol{\partial} H \cdot \mathbb{A} \boldsymbol{\xi}$, where $\boldsymbol{\partial}$ is defined by formula (9.2). Substituting (9.58) and (9.59) into (9.57) we arrive at the identity

$$
\begin{aligned}
\Pi\left(\mathfrak{L}+\mathfrak{B}_{0}+\mathfrak{F}_{2,0}\right) \Pi \mathfrak{H} \mathcal{D}^{-1} \Pi & =\Pi \mathfrak{Q}_{3} \mathfrak{L} \Pi+2 \nu \Pi \mathfrak{H}_{1} \Pi+ \\
& +\Pi \mathfrak{B}_{3} \Pi \mathcal{D}^{-1}+\Pi \mathfrak{F}_{2,1} \Pi,
\end{aligned}
$$

where the zero order operator $\mathfrak{B}_{3}$ has the symbol

$$
B_{3}(Y, \boldsymbol{\xi})=\nu \mathcal{D}^{2} H(Y, \boldsymbol{\xi})+B_{0}(Y, \boldsymbol{\xi}) H(Y, \boldsymbol{\xi})-i \boldsymbol{\partial} H(Y, \boldsymbol{\xi}) \cdot \mathbb{A} \boldsymbol{\xi}
$$

the remainder $\mathfrak{F}_{2,1}$ and operator $\mathfrak{Q}_{3}$ are equal to

$$
\begin{aligned}
& \mathfrak{F}_{2,1}=\Pi \mathfrak{F}_{2,0} \Pi \mathfrak{H} \Pi \mathcal{D}^{-1}+\Pi\left(\mathfrak{B}_{0} \mathfrak{Z}+\mathfrak{D}_{1}^{\left(\mathfrak{B}_{0} \mathfrak{H}\right)}+\mathfrak{D}_{2}^{\left[(-\Delta)^{-1 / 2}, \mathfrak{H}\right]}\right) \Pi \mathcal{D}^{-1} \\
& \mathfrak{Q}_{3}=\Pi\left(\mathfrak{H}+\mathfrak{B}_{0} \mathfrak{Y}\right) \Pi \mathcal{D}^{-1}
\end{aligned}
$$

Identity (9.60) obviously implies

$$
\begin{aligned}
& \Pi\left(\mathfrak{L}+\mathfrak{B}_{0}+\mathfrak{F}_{2,0}\right)\left(\mathbf{I}+\Pi \mathfrak{H D}^{-1} \Pi\right) \Pi=\Pi\left(2 \nu \mathfrak{H}_{1}+\mathfrak{B}_{0}\right) \Pi+ \\
& +\left(\mathbf{I}+\Pi \mathfrak{Q}_{3}\right) \mathfrak{L} \Pi+\Pi \mathfrak{B}_{3} \Pi \mathcal{D}^{-1}+\Pi\left(\mathfrak{F}_{2,0}+\mathfrak{F}_{2,1}\right) \Pi .
\end{aligned}
$$

Next note that the symbol $B_{0}(Y, \boldsymbol{\xi})-V(\boldsymbol{\xi})$ has a zero mean value over a period and we can take

$$
H(Y, \boldsymbol{\xi})=-(2 \nu)^{-1} \mathcal{D}^{-1}\left(B_{0}(Y, \boldsymbol{\xi})-V(\boldsymbol{\xi})\right)
$$

to obtain $2 \nu \mathfrak{H}_{1}+\mathfrak{B}_{0}=\mathfrak{V}$, which gives

$$
\begin{aligned}
& \Pi\left(\mathfrak{L}+\mathfrak{B}_{0}+\mathfrak{F}_{2,0}\right)\left(\mathbf{I}+\Pi \mathfrak{H D}^{-1} \Pi\right) \Pi=\Pi \mathfrak{V} \Pi+ \\
& +\left(\mathbf{I}+\Pi \mathfrak{Q}_{3}\right) \mathfrak{L} \Pi+\Pi \mathfrak{B}_{3} \Pi \mathcal{D}^{-1}+\Pi\left(\mathfrak{F}_{2,0}+\mathfrak{F}_{2,1}\right) \Pi .
\end{aligned}
$$

Next note that $\mathfrak{Q}_{3}=\Pi \mathfrak{Q}_{3}=\mathfrak{Q}_{3} \Pi$, and $\mathfrak{V}$ commutes with $\Pi$, which along with the expression for $\mathfrak{Q}_{3}$ gives

$$
-\mathfrak{Q}_{3} \Pi \mathfrak{V} \Pi=-\left(\mathbf{I}+\mathfrak{Q}_{3}\right) \Pi \mathfrak{H} \mathfrak{V} \Pi \mathcal{D}^{-1}+\mathfrak{Q}_{3} \Pi \mathfrak{H} \mathfrak{V} \Pi \mathcal{D}^{-1}-\Pi \mathfrak{B}_{0} \mathfrak{Y} \mathcal{V}^{-1} \Pi .
$$

This leads to the identity

$$
\begin{aligned}
& \Pi\left(\mathfrak{L}+\mathfrak{B}_{0}+\mathfrak{F}_{2,0}\right)\left(\mathbf{I}+\Pi \mathfrak{H} \mathcal{D}^{-1} \Pi\right) \Pi=\left(\mathbf{I}+\mathfrak{Q}_{3}\right) \Pi\left(\mathfrak{L}+\mathfrak{V}+\left(\mathfrak{B}_{3}-\mathfrak{H} \mathfrak{V}\right) \mathcal{D}^{-1}\right) \Pi+ \\
& +\Pi\left(\mathfrak{F}_{2,0}+\mathfrak{F}_{2,1}\right) \Pi+\Pi\left(\mathfrak{Q}_{3} \mathfrak{H}-\mathfrak{B}_{0} \mathfrak{Y}\right) \mathfrak{V D}^{-1} \Pi-\Pi \mathfrak{Q}_{3} \mathfrak{B}_{3} \mathcal{D}^{-1} \Pi .
\end{aligned}
$$


Now set

$$
\begin{aligned}
& \mathfrak{F}_{2,2}=\Pi\left(\mathfrak{F}_{2,0}+\mathfrak{F}_{2,1}\right) \Pi+\Pi\left(\mathfrak{Q}_{3} \mathfrak{H}-\mathfrak{B}_{0} \mathfrak{Y}\right) \mathfrak{V} \mathcal{D}^{-1} \Pi-\Pi \mathfrak{Q}_{3} \mathfrak{B}_{3} \mathcal{D}^{-1} \Pi \\
& \mathfrak{F}_{2}=\left(\mathbf{I}+\Pi \mathfrak{Q}_{3}\right)^{-1} \mathfrak{F}_{2,2}
\end{aligned}
$$

and $B_{2}=B_{3}-H V$. Thus we can rewrite the last identity in the equivalent form

$$
\begin{aligned}
& \Pi\left(\mathfrak{L}+\mathfrak{B}_{0}+\mathfrak{F}_{2,0}\right)\left(\Pi+\Pi \mathfrak{H} \mathcal{D}^{-1} \Pi\right)= \\
& =\left(\mathbf{I}+\mathfrak{Q}_{3}\right) \Pi\left(\mathfrak{L}+\mathfrak{V}+\mathfrak{B}_{2} \mathcal{D}^{-1}+\mathfrak{F}_{2}\right) \Pi,
\end{aligned}
$$

where $\mathfrak{B}_{2}$ is a zero order operator with the symbol $B_{2}$. Our next task is to define operators $\mathfrak{S}_{2}$ and $\mathfrak{T}_{2}$. To do this we multiply (from the right) both sides of identity $(9.56)$ by the operator

$$
\mathbf{I}+\Pi \mathfrak{H D}^{-1} \Pi=\Pi+\Pi \mathfrak{H} \mathcal{D}^{-1} \Pi+(\mathbf{I}-\Pi) .
$$

By virtue of (9.64) and the trivial identity $(\mathbf{I}-\Pi) \mathfrak{L} \Pi=\mathfrak{L}(\mathbf{I}-\Pi) \Pi=0$, the result of this multiplication can be written in the form

$$
\begin{aligned}
& \left(\mathbf{I}-\Pi \mathfrak{Q}_{2}\right)\left(\mathfrak{L}+\mathfrak{B}_{0}+\mathfrak{F}_{0}\right)\left(\mathbf{I}+\Pi \mathfrak{H D}^{-1} \Pi\right)= \\
& =\left(\mathbf{I}+\Pi \mathfrak{Q}_{3}\right) \Pi\left(\mathfrak{L}+\mathfrak{V}+\mathfrak{B}_{2} \mathcal{D}^{-1}+\mathfrak{F}_{2}\right) \Pi+(\mathbf{I}-\Pi)\left(\mathfrak{L}+\mathfrak{P}_{2}\right),
\end{aligned}
$$

where

$$
\mathfrak{P}_{2}=\left(\mathfrak{B}_{0}+\mathfrak{F}_{0}\right)\left(\mathbf{I}+\Pi \mathfrak{H} \mathcal{D}^{-1} \Pi\right) .
$$

Notice that $\left(\mathbf{I}+\mathfrak{Q}_{3}\right)^{-1}(\mathbf{I}-\Pi)=\mathbf{I}-\Pi$ since $\mathfrak{Q}_{3}=\Pi \mathfrak{Q}_{3} \Pi$. Thus we arrive at the desired equality (9.51) with the operators

$$
\mathfrak{S}_{2}=\left(\mathbf{I}+\Pi \mathfrak{Q}_{3} \Pi\right)^{-1}\left(\mathbf{I}-\Pi \mathfrak{Q}_{2}\right), \quad \mathfrak{T}_{2}=\left(\mathbf{I}+\Pi \mathfrak{H} \mathcal{D}^{-1} \Pi\right) .
$$

It remains to prove inequalities (9.52)- (9.54) and, by doing so, to justify the above algebraic construction. We begin with the observation that estimate (9.26) with $i=0$ for the symbol $B_{0}$ along with the tame estimate (8.19) in Lemma 9 implies the inequality

$$
\left\|\mathfrak{B}_{0} u\right\|_{s} \leq c \epsilon^{1 / 2}\|u\|_{s}
$$

for all $u \in H_{o}^{s}, s \in[0, r-13]$. It follows from this and inequalities (9.37) and (8.27) that

$$
\|\mathfrak{K} u\|_{s+1} \leq c \epsilon^{1 / 2}\|u\|_{s}
$$

for all $u \in H_{o}^{s}, s \in[0, r-15]$. Applying Lemma 11 we conclude that there is $\epsilon_{0}>0$, depending only on the constant $c_{0}$ in conditions $($ H.1)- (H.2) and exponent $s$, so that

$$
\left\|(\mathbf{I}-\mathfrak{K}(\mathbf{I}-\Pi))^{-1} u\right\|_{s} \leq c\|u\|_{s}
$$

for all $\epsilon \in\left[0, \epsilon_{0}\right], u \in H_{o}^{s}, s \in[0, r-15]$. In particular we obtain from this, (9.37), and (8.27) that

$$
\left\|\mathfrak{Q}_{2} u\right\|_{s} \leq c \epsilon^{1 / 2}\|u\|_{s}, \quad\left\|\mathfrak{F}_{2,0} u\right\|_{s+1} \leq c \epsilon^{1 / 2}\|u\|_{s},
$$


for all $u \in H_{o}^{s}, s \in[0, r-15]$. Let us now estimate the operator $\mathfrak{H}$. Recalling estimate (9.28) we obtain from formula (9.62) that

$$
|H|_{r-11} \leq c \epsilon^{1 / 2}
$$

which along with Lemma 9 implies

$$
\|\mathfrak{H} u\|_{s} \leq c \epsilon^{1 / 2}\|u\|_{s}
$$

for all $u \in H_{o}^{s}, s \in[0, r-16]$. Moreover, estimate (8.22) for compositions and estimate (8.24) for commutators in Lemma 9 along with estimates (9.26) and (9.69) for the symbols $B_{0}$ and $H$ give

$$
\left\|\mathfrak{D}_{1}^{\left(\mathfrak{B}_{0} \mathfrak{H}\right)} u\right\|_{s+1}+\left\|\mathfrak{D}_{2}^{\left[(-\Delta)^{-1 / 2}, \mathfrak{H}\right]} u\right\|_{s+1} \leq c \epsilon^{1 / 2}\|u\|_{s}
$$

for all $u \in H_{o}^{s}, s \in[0, r-18]$. On the other hand, operator $\mathfrak{H}$ meets all requirements of Lemma 10 with $r$ replaced by $r-11$. This leads to the estimate for the operators $\mathfrak{Y}$ and $\mathfrak{Z}$,

$$
\|\mathfrak{Y} u\|_{s+1}+\|\mathfrak{Z} u\|_{s+1} \leq c \epsilon^{1 / 2}\|u\|_{s}
$$

for all $u \in H_{o}^{s}, s \in[0, r-16)$. Since $\left\|\mathcal{D}^{-1} \Pi u\right\|_{s+1 / 2} \leq c(s)\|u\|_{s}$ for all $s \geq 0$, we conclude from this, estimates (9.68), (9.71), and formula for $\mathfrak{F}_{2,1}$ that

$$
\left\|\mathfrak{F}_{2,1} u\right\|_{s+1} \leq c \epsilon^{1 / 2}\|u\|_{s},
$$

for all $u \in H_{o}^{s}, s \in[0, r-18]$. Similarly, formula for $\mathfrak{Q}_{3}$ along with inequalities (9.67) for $\mathfrak{B}_{0},(9.70)$ for $\mathfrak{H}$ and $(9.72)$ for $\mathfrak{Y}$ implies that the inequality

$$
\left\|\mathfrak{Q}_{3} u\right\|_{s+1 / 2} \leq c \epsilon^{1 / 2}\|u\|_{s},
$$

holds true for all $u \in H_{o}^{s}, s \in[0, r-33 / 2]$.

Applying Lemma 11 we conclude that there is $\epsilon_{0}>0$, depending only on constant $c_{0}$, and $s$, so that

$$
\left\|\left(\mathbf{I}-\mathfrak{Q}_{3}\right)^{-1} u\right\|_{s} \leq c \epsilon^{1 / 2}\|u\|_{s}
$$

holds true for all $u \in H_{o}^{s}, s \in[0, r-33 / 2]$, and $\epsilon \in\left[0, \epsilon_{0}\right]$. Formula (9.61) for the symbol $B_{3}$ along with estimate (9.69) lead to the inequality

$$
\left|B_{3}\right|_{r-13} \leq c \epsilon^{1 / 2}
$$

which along with Lemma 9 implies the tame estimate

$$
\left\|\mathfrak{B}_{3} u\right\|_{s} \leq c \epsilon^{1 / 2}\|u\|_{s},
$$

for all $u \in H_{o}^{s}, s \in[0, r-18]$. This gives

$$
\left\|\mathfrak{B}_{3} \Pi \mathcal{D}^{-1} u\right\|_{s+1 / 2} \leq c \epsilon^{1 / 2}\left\|\Pi \mathcal{D}^{-1} u\right\|_{s+1 / 2} \leq c \epsilon^{1 / 2}\|u\|_{s},
$$

for all $u \in H_{o}^{s}, s \in[0, r-37 / 2]$. Combining this result with (9.74) we conclude that for all $u \in H_{o}^{s}, s \in[0, r-37 / 2]$,

$$
\left\|\mathfrak{Q}_{3} \mathfrak{B}_{3} \Pi \mathcal{D}^{-1} u\right\|_{s+1} \leq c \epsilon^{1 / 2}\|u\|_{s} .
$$


Repeating these arguments and using tame estimate $(9.70)$ for $\mathfrak{H}$ instead of estimate (9.77) for $\mathfrak{B}_{3}$ we obtain for all $u \in H_{o}^{s}, s \in[0, r-37 / 2]$

$$
\left\|\mathfrak{Q}_{3} \mathfrak{H} \Pi \mathcal{D}^{-1} u\right\|_{s+1} \leq c \epsilon^{1 / 2}\|u\|_{s} .
$$

Recall that $\mathfrak{V}$ is a zero order differential operator with constant symbol, which gives

$$
\left\|\mathfrak{V} \Pi \mathcal{D}^{-1} u\right\|_{s} \leq c(s)\|u\|_{s} \text { for all } s \geq 0 .
$$

From this and (9.72), (9.67) we conclude that for all $u \in H_{o}^{s}, s \in[0, r-16]$,

$$
\left\|\mathfrak{B}_{0} \mathfrak{Y} \mathfrak{V} \Pi \mathcal{D}^{-1} u\right\|_{s+1} \leq c \epsilon^{1 / 2}\|u\|_{s} .
$$

Substituting inequalities (9.68), (9.73), and (9.78)-(9.80) into (9.63) we obtain for all $u \in H_{o}^{s}, s \in[0, r-37 / 2]$,

$$
\left\|\mathfrak{F}_{2,2} u\right\|_{s+1} \leq c \epsilon^{1 / 2}\|u\|_{s} .
$$

Combining this result with estimate $(9.75)$ we obtain the desired inequality (9.53). Next, Lemma 11 along with formulae (9.66) and inequalities (9.68), (9.70), (9.74) implies estimate (9.54) for the operators $\mathfrak{S}_{2}$ and $\mathfrak{T}_{2}$. In its turn, formula (9.65) for the operator $\mathfrak{P}_{2}$ along with inequalities $(9.37),(9.67)$, and (9.70) yields estimate (9.53) for $\mathfrak{P}_{2}$. Finally notice that by virtue of $(9.62)$ we have $B_{0}-V=-2 \nu \mathcal{D} H$. Recalling the equality $B_{2}=B_{3}-H V$ and formula (9.61) we conclude from this that

$$
B_{2}(Y, \boldsymbol{\xi})=\nu \mathcal{D}\left(\mathcal{D} H(Y, \boldsymbol{\xi})-H(Y, \boldsymbol{\xi})^{2}\right)-i \boldsymbol{\partial} H(Y, \boldsymbol{\xi}) \cdot \mathbb{A} \boldsymbol{\xi}
$$

Hence $B_{2}$ has a zero mean over a period. It remains to note that estimate (9.52) is a straightforward consequence of inequality (9.69) and the proposition follows.

Now our task is to make the last step in the descend method to eliminate the term $\mathfrak{B}_{2} \mathcal{D}^{-1}$ in the operator $\mathfrak{L}+\mathfrak{V}+\mathfrak{B}_{2} \mathcal{D}^{-1}+\mathfrak{F}_{2}$ in Proposition 3. This result is given by the following

third step Proposition 4 Let $s \in[0, r-24]$ and suppose that operators $\mathfrak{B}_{2} \mathfrak{F}_{2}$ meet all requirements of Proposition 3. Then there exists $\epsilon_{0}$, depending only on the constant $c_{0}$ in Conditions $(\boldsymbol{H . 1})-(\boldsymbol{H} .2)$ and on the exponent $s$, with the following properties. For any $\epsilon \in\left(0, \epsilon_{0}\right)$, there are bounded operators $\mathfrak{S}_{4}, \mathfrak{T}_{4}, \mathfrak{P}_{4}: H_{o}^{s} \mapsto H_{o}^{s}, \mathfrak{F}_{4}: H_{o}^{s} \mapsto H_{o}^{s+1}$ so that

$$
\begin{aligned}
\mathfrak{S}_{4}(\Pi(\mathfrak{L}+\mathfrak{V} & \left.\left.+\mathfrak{B}_{2} \mathcal{D}^{-1}+\mathfrak{F}_{2}\right) \Pi+(\mathbf{I}-\Pi)\left(\mathfrak{L}+\mathfrak{P}_{2}\right)\right) \mathfrak{T}_{4}= \\
& =\Pi\left(\mathfrak{L}+\mathfrak{V}+\mathfrak{F}_{4}\right) \Pi+(\mathbf{I}-\Pi)\left(\mathfrak{L}+\mathfrak{P}_{4}\right) .
\end{aligned}
$$

For all $u \in H_{o}^{s}$, operators $\mathfrak{F}_{4}, \mathfrak{S}_{4}, \mathfrak{T}_{4}$, and $\mathfrak{P}_{4}$ satisfy the inequalities

$$
\begin{gathered}
\left\|\mathfrak{P}_{4} u\right\|_{s}+\left\|\mathfrak{F}_{4} u\right\|_{s+1} \leq c \epsilon^{1 / 2}\|u\|_{s} \\
\left\|\left(\mathfrak{S}_{4}^{ \pm 1}-\mathbf{I}\right) u\right\|_{s}+\left\|\left(\mathfrak{T}_{4}^{ \pm 1}-\mathbf{I}\right) u\right\|_{s} \leq c \epsilon^{1 / 2}\|u\|_{s}
\end{gathered}
$$


Proof The proof imitates the proof of Proposition 3 with essential simplifications. In order to avoid repetitions we give only the proof of the algebraic part of the proposition. Choose as before an arbitrary zero order operator $\mathfrak{H}$ with a smooth symbol $H(Y, \boldsymbol{\xi})$. We have

$$
\begin{aligned}
\Pi \mathfrak{L} \Pi \mathfrak{H} \mathcal{D}^{-2} \Pi=\Pi \mathfrak{H D}^{-2} \mathfrak{L} \Pi+2 \nu \Pi \mathfrak{H}_{1} \Pi \mathcal{D}^{-1} & +\nu \Pi \mathfrak{H}_{11} \mathcal{D}^{-2} \Pi+ \\
& +\Pi\left[(-\Delta)^{1 / 2}, \mathfrak{H}\right] \mathcal{D}^{-2} \Pi,
\end{aligned}
$$

which gives

$\Pi\left(\mathfrak{L}+\mathfrak{V}+\mathfrak{B}_{2} \mathcal{D}^{-1}+\mathfrak{F}_{2}\right) \Pi \mathfrak{H} \mathcal{D}^{-2} \Pi=\Pi \mathfrak{H D}^{-2} \mathfrak{L} \Pi+2 \nu \Pi \mathfrak{H}_{1} \mathcal{D}^{-1} \Pi+\Pi \mathfrak{F}_{4,0} \Pi$, where the operator $\mathfrak{F}_{4,0}$ is defined by

$\mathfrak{F}_{4,0}=\nu \Pi \mathfrak{H}_{11} \mathcal{D}^{-2} \Pi+\Pi\left[(-\Delta)^{1 / 2}, \mathfrak{H}\right] \mathcal{D}^{-2} \Pi+\Pi\left(\mathfrak{V}+\mathfrak{B}_{2} \mathcal{D}^{-1}+\mathfrak{F}_{2}\right) \mathfrak{H D}^{-2} \Pi$.

This leads to the identity

$$
\begin{aligned}
& \Pi\left(\mathfrak{L}+\mathfrak{V}+\mathfrak{B}_{2} \mathcal{D}^{-1}+\mathfrak{F}_{2}\right)\left(\Pi+\Pi \mathfrak{H D}^{-2} \Pi\right)= \\
& =\Pi\left(\mathbf{I}+\mathfrak{H D}^{-2} \Pi\right)(\mathfrak{L}+\mathfrak{V}) \Pi+\Pi\left(\mathfrak{B}_{2}+2 \nu \Pi \mathfrak{H}_{1}\right) \mathcal{D}^{-1} \Pi+ \\
& +\Pi\left(\mathfrak{F}_{2}+\mathfrak{F}_{4,0}-\Pi \mathfrak{H} \mathfrak{V} \mathcal{D}^{-2}\right) \Pi .
\end{aligned}
$$

Here we use the fact that $\mathfrak{V}$ commutes with $\Pi$. Setting $H=-(2 \nu)^{-1} \mathcal{D}^{-1} B_{2}$ we obtain

$\Pi\left(\mathfrak{L}+\mathfrak{V}+\mathfrak{B}_{2} \mathcal{D}^{-1}+\mathfrak{F}_{2}\right)\left(\Pi+\Pi \mathfrak{H} \mathcal{D}^{-2} \Pi\right)=\Pi\left(\mathbf{I}+\Pi \mathfrak{H} \mathcal{D}^{-2} \Pi\right)\left(\mathfrak{L}+\mathfrak{V}+\mathfrak{F}_{4}\right) \Pi$.

where

$$
\mathfrak{F}_{4}=\left(\mathbf{I}+\Pi \mathfrak{H} \mathcal{D}^{-2} \Pi\right)^{-1} \Pi\left(\mathfrak{F}_{2}+\mathfrak{F}_{4,0}-\Pi \mathfrak{H} \Pi \mathfrak{V} \mathcal{D}^{-2}\right) \Pi .
$$

Thus we get

$$
\begin{aligned}
& \left\{\Pi\left(\mathfrak{L}+\mathfrak{V}+\mathfrak{B}_{2} \mathcal{D}^{-1}+\mathfrak{F}_{2}\right) \Pi+(\mathbf{I}-\Pi)\left(\mathfrak{L}+\mathfrak{P}_{2}\right)\right\}\left(\mathbf{I}+\Pi \mathfrak{H} \mathcal{D}^{-2} \Pi\right)= \\
& \left.\quad=\Pi\left(\mathbf{I}+\Pi \mathfrak{H} \mathcal{D}^{-2} \Pi\right)\left(\mathfrak{L}+\mathfrak{V}+\mathfrak{F}_{4}\right) \Pi+(\mathbf{I}-\Pi)\left(\mathfrak{L}+\mathfrak{P}_{2}\right)\right\}\left(\mathbf{I}+\Pi \mathfrak{H} \mathcal{D}^{-2} \Pi\right) .
\end{aligned}
$$

Noting that for $\mathfrak{P}_{4}=\mathfrak{P}_{2}\left(\mathbf{I}+\Pi \mathfrak{H} \mathcal{D}^{-2} \Pi\right)$, we have

$$
(\mathbf{I}-\Pi)\left(\mathfrak{L}+\mathfrak{P}_{2}\right)\left(\mathbf{I}+\Pi \mathfrak{H} \mathcal{D}^{-2} \Pi\right)=(\mathbf{I}-\Pi)\left(\mathfrak{L}+\mathfrak{P}_{4}\right),
$$

and using the identity $\left(\mathbf{I}+\Pi \mathfrak{H} \mathcal{D}^{-2} \Pi\right)^{-1}(\mathbf{I}-\Pi)=\mathbf{I}-\Pi$, we arrive at the representation (9.81) with

$$
\mathfrak{S}_{4}=\left(\mathbf{I}+\Pi \mathfrak{H} \mathcal{D}^{-2} \Pi\right)^{-1}, \quad \mathfrak{T}_{4}=\mathbf{I}+\Pi \mathfrak{H} \mathcal{D}^{-2} \Pi .
$$

Next, by virtue of (9.52), we have $|H|_{r-16} \leq c \epsilon^{1 / 2}$. Combining this result with estimates (9.53)-(9.54) in Proposition 3 and arguing as in the proof of this proposition we obtain (9.82)-(9.83).

Finally notice that if we define the operators $\mathfrak{S}, \mathfrak{T}, \mathfrak{F}$ and $\mathfrak{P}$ in theorem 8 by the equalities

$$
\mathfrak{S}=\mathfrak{S}_{4} \mathfrak{S}_{2} \mathfrak{S}_{0} \mathfrak{S}_{-1}, \quad \mathfrak{T}=\mathfrak{T}_{4} \mathfrak{T}_{2} \mathfrak{T}_{0}, \quad \mathfrak{F}=\mathfrak{F}_{4}, \quad \mathfrak{P}=\mathfrak{P}_{4},
$$

then the assertion of Theorem 8 directly follows from Propositions 1-4. 


\section{$10 \mathfrak{L}$ and $\mathcal{D}$ resolvents}

Recall that our goal is to find a family of solutions in the form (6.3) to the system of nonlinear equations (6.1), and we intend to obtain this result by using the Nash-Moser iteration scheme. In this framework the main task is to prove the solvability of the truncated equation (7.29). By virtue of Theorem 8 this problem is equivalent to the problem of the invertibility of the operator in the right hand side of relation (9.19). Notice that this relation is an algebraic identity, and Theorem 8 alone does not provide the invertibility of operators in (9.19). In this section we investigate in details the question on the invertibility of the pseudodifferential operators

$$
\mathfrak{L} \equiv \nu \mathcal{D}^{2}+(-\Delta)^{1 / 2}, \quad \mathfrak{L}+\mathfrak{V}, \quad \text { and } \mathcal{D}=\varrho \cdot \nabla
$$

involved in (9.19). Recall that they have the symbols

$$
L(\mathbf{k})=(\mathbb{A} \widetilde{\mathbf{k}} \cdot \widetilde{\mathbf{k}})^{1 / 2}-\nu\left(k_{1}+\rho k_{2}\right)^{2}, L(\mathbf{k})+V(\mathbf{k}) \text { and } D(\mathbf{k})=i\left(k_{1}+\rho k_{2}\right)
$$

which do not depend on $Y$, i. e., they are operators with constant coefficients. Here the quantities $\rho$ and $\nu$ are given parameters, but the matrix $\mathbb{A}$ and symbol $V$ depend on the point of linearization $(U, X, \mu, \mathbf{u})$ and are determined by formulae (9.6) and (9.15). With application to the implicit function Theorem 14 in mind we consider the question on the simultaneous invertibility of the sequence of operators $\mathcal{L}_{q}$ and $\mathfrak{L}_{q}+\mathfrak{V}_{q}, q \geq 1$ corresponding to functions and parameters $\left(U_{q}, X_{q}, \rho, \nu, \mu_{q}, \mathbf{u}_{q}\right)$ satisfying the following condition.

lition13.1bis Condition 9 Functions $\left(U_{q}, X_{q}\right)$ and parameters $\rho, \nu, \mu_{q}, \mathbf{u}_{q}$ admit the representation

$$
\begin{aligned}
& U_{q}=U_{4 m}(\varepsilon)+|\varepsilon|^{2 m} \breve{U}_{q}(\rho, \nu), \quad X_{q}=X_{4 m}(\varepsilon)+|\varepsilon|^{2 m} \breve{\mathcal{W}}_{q}(\rho, \nu), \\
& \mathbf{u}_{q}(\varepsilon)=\mathbf{u}_{4 m}(\varepsilon)+|\varepsilon|^{2 m} \breve{\boldsymbol{\omega}}_{q}(\rho, \nu), \quad \mu_{q}=\mu_{4 m}(\varepsilon)+|\varepsilon|^{2 m} \breve{\mu}_{q}(\rho, \nu), \\
& \rho=\rho_{4 m}(\varepsilon), \quad \nu=\nu_{4 m}(\varepsilon), \quad(\rho, \nu) \in \omega_{\epsilon_{0}},
\end{aligned}
$$

where $\left(U_{4 m}, X_{4 m}, \rho_{4 m}, \nu_{4 m}, \mu_{4 m}, \mathbf{u}_{4 m}\right)$ is an approximate solution of Problem 1 determined by Theorem 4 and Lemma 5, the domain $\omega_{\epsilon_{0}}$ is defined in Lemma 6. By virtue of Lemma 5, we shall consider $\varepsilon$ as a fixed analytic function of $(\rho, \nu)$ defined in $\omega_{\epsilon_{0}}$ and satisfying the inequalities

$$
|\varepsilon| \leq c\left(|\rho-\lambda|+\left|\nu-\nu_{c}\right|\right)^{1 / 2}, \quad\left|\partial_{(\rho, \nu)} \varepsilon\right| \leq c\left(|\rho-\lambda|+\left|\nu-\nu_{c}\right|\right)^{-1 / 2}
$$

Notice that $\varepsilon_{i}^{2}, i=1,2$, are analytic functions of $(\rho, \nu)$ defined in a vicinity of $\left(\lambda, \nu_{c}\right)$. We assume that there are $r \geq 26$ and $c>0$ such that

$$
\begin{aligned}
\left\|\breve{U}_{q}\right\|_{C^{r}\left(\mathbb{R}^{2} / \Gamma\right)} & +\left\|\breve{\mathcal{W}}_{q}\right\|_{C^{r}\left(\mathbb{R}^{2} /(2 \pi \mathbb{Z})^{2}\right)}+\left|\breve{\omega}_{q}\right|+\left|\breve{\mu}_{q}\right| \leq c, \\
\left\|\breve{U}_{q}-\breve{U}_{q+1}\right\|_{C^{r}\left(\mathbb{R}^{2} / \Gamma\right)} & +\left\|\breve{\mathcal{W}}_{q}-\breve{\mathcal{W}}_{q+1}\right\|_{C^{r}\left(\mathbb{R}^{2} /(2 \pi \mathbb{Z})^{2}\right)}+ \\
& +\left|\breve{\omega}_{q}-\breve{\omega}_{q+1}\right|+\left|\breve{\mu}_{q}-\breve{\mu}_{q}\right| \leq c 2^{-q},
\end{aligned}
$$




$$
\begin{aligned}
& \text { and for every }(\rho, \nu),\left(\rho^{\prime}, \nu\right) \in \omega_{\epsilon_{0}} \text {, } \\
& \qquad \begin{array}{l}
\left\|\breve{U}_{q}(\rho, \nu)-\breve{U}_{q}\left(\rho^{\prime}, \nu\right)\right\|_{C^{r}\left(\mathbb{R}^{2} / \Gamma\right)}+\left\|\breve{\mathcal{W}}_{q}(\rho, \nu)-\breve{\mathcal{W}}_{q}\left(\rho^{\prime}, \nu\right)\right\|_{C^{r}\left(\mathbb{R}^{2} /(2 \pi \mathbb{Z})^{2}\right)}+ \\
\quad+\left|\breve{\omega}_{q}(\rho, \nu)-\breve{\omega}_{q}\left(\rho^{\prime}, \nu\right)\right|+\left|\breve{\mu}_{q}(\rho, \nu)-\breve{\mu}_{q}\left(\rho^{\prime}, \nu\right)\right| \leq c\left|\rho-\rho^{\prime}\right| .
\end{array}
\end{aligned}
$$

Denote by $\mathbb{A}_{q}$ and $V_{q}$ the matrices and symbols corresponding to $\left(U_{q}, X_{q}, \rho, \nu, \mu_{q}, \mathbf{u}_{q}\right)$. For $\varepsilon=0$ (equivalently $\rho=\lambda, \nu=\nu_{c}$ ) all matrices $\mathbb{A}_{q}$ coincide and are equal to the matrix $\mathbb{A}_{c}=\mathbb{T}^{*-1} \mathbb{T}^{-1}$, where $\mathbb{T}$ is determined by (5.1). We shall use the following notations for their elements

$$
\mathbb{A}_{q}=\left(\begin{array}{cc}
b_{q} & a_{q} \\
a_{q} & 1
\end{array}\right) \quad \mathbb{A}_{c}=:\left.\mathbb{A}_{q}\right|_{\varepsilon=0}=\left(\begin{array}{cc}
b_{c} & a_{c} \\
a_{c} & 1
\end{array}\right) .
$$

It follows from formula (5.1) and the expressions for vectors $\boldsymbol{\lambda}_{i}$, which generate the lattice $\Gamma$, that

$$
\begin{aligned}
& a_{c}=-\tau_{1}\left(1+\tau_{1}^{2}\right)^{1 / 2}\left(1+\tau_{2}^{2}\right)^{-1 / 2}\left(\tau_{1}+\tau_{2}\right)^{-1}, \\
& b_{c}=\left(1+\tau_{1}^{2}\right)^{2}\left(1+\tau_{2}^{2}\right)^{-1}\left(\tau_{1}+\tau_{2}\right)^{-2} .
\end{aligned}
$$

By virtue of (10.3) $U_{4 m}$ and $X_{4 m}$ as functions of parameters $(\rho, \nu)$ have a branching point at $\left(\lambda, \nu_{c}\right)$. However, the following lemma shows that the corresponding matrix $\mathbb{A}$ and symbol $V$ are analytic at this point.

Lend(rho,nu)2 Lemma 13 In taking $(U, X, \mathbf{u}, \mu)=\left(U_{4 m}, X_{4 m}, \mathbf{u}_{4 m}, \mu_{4 m}\right)(\varepsilon)$, and $\rho=$ $\rho_{4 m}(\varepsilon), \nu=\nu_{4 m}(\varepsilon)$, the coefficients $a_{q}, b_{q}$ and the symbol $V_{q}$ of $\mathfrak{V}_{q}$ take the form

$$
a_{4 m}^{(0)}(\rho, \nu), b_{4 m}^{(0)}(\rho, \nu), V_{4 m}^{(0)}(\rho, \nu, \mathbf{k})
$$

which are analytic functions of $(\rho, \nu) \in \omega_{\epsilon_{0}}$.

Proof The coefficients $g_{i j}$ of the matrix $\mathbb{G}$ computed with the approximate solution of order $|\varepsilon|^{4 m}$ are invariant under the symmetries $\widetilde{\mathcal{S}}_{j}$, as this results from Theorem 4, and from Lemma 5 . This gives coefficients $G_{1, i j}$ in (9.3) also invariants under $\widetilde{\mathcal{S}}_{j}$, hence the matrix $\mathbb{A}$ defined by (9.6) is also invariant under $\widetilde{\mathcal{S}}_{j}$. Since its coefficients $(a, b)$ are independent of $Y$ it results that these coefficients are invariant under the changes $\varepsilon_{1} \mapsto-\varepsilon_{1}$ and independently $\varepsilon_{2} \mapsto-\varepsilon_{2}$, which means that they only depend on $\left(\varepsilon_{1}^{2}, \varepsilon_{2}^{2}\right)$. Then the Lemma 6 allows to conclude for $a_{4 m}^{(0)}(\rho, \nu)$ and $b_{4 m}^{(0)}(\rho, \nu)$.

Now, the invariance under $\widetilde{\mathcal{S}}_{j}$ of the functions $p(Y)$ and $\mathbb{B}_{12}(Y)$ (computed with the approximate solution at order $|\varepsilon|^{4 m}$ ), gives the same property for $A(Y, \boldsymbol{\xi}), W(Y, \boldsymbol{\xi}), B_{1}(Y, \boldsymbol{\xi}), E(Y, \boldsymbol{\xi})$ as defined by $(9.9),(9.13)$ and (9.14). The same property holds for $Q_{0}(Y, \boldsymbol{\xi})$ (see $(9.11)$ ) and $J_{0}(Y, \boldsymbol{\xi})$ (see (9.5), hence it holds for $B(Y, \boldsymbol{\xi})$ defined by (9.10). It then results that $V_{4 m}^{(0)}(\rho, \nu, \mathbf{k})$ which is $V(\boldsymbol{\xi})$ defined by (9.15) and computed with the approximate solution at order $|\varepsilon|^{4 m}$ only depends on $\left(\varepsilon_{1}^{2}, \varepsilon_{2}^{2}\right)$. We conclude as for coefficients $a$ and $b$.

Since, by virtue of (10.3), the function $|\varepsilon(\rho, \nu)|^{2 m}, m>1$, has a continuous derivatives in $\omega_{\epsilon_{0}}$, we arrive at the following 
Lemma 14 Let functions $\left(U_{q}, X_{q}\right)$ and parameters $\rho, \nu, \mu_{q}, \mathbf{u}_{q}$ meet all requirements of Condition 9. Then for any $(\rho, \nu) \in \omega_{\epsilon_{0}}$ and $m>1$, the real quantities $a_{q}, b_{q}$ admit the representation

$$
a_{q}=a_{4 m}^{(0)}(\rho, \nu)+|\varepsilon|^{2 m} \check{a}_{q}(\rho, \nu), \quad b_{q}=b_{4 m}^{(0)}(\rho, \nu)+|\varepsilon|^{2 m} \check{b}_{q}(\rho, \nu),
$$

such that $a_{4 m}^{(0)}\left(\lambda, \nu_{c}\right)=a_{c}$ and $b_{4 m}^{(0)}\left(\lambda, \nu_{c}\right)=b_{c}$. Furthermore, the functions $\check{a}_{q}, \check{b}_{q}$ satisfy the inequalities

$$
\begin{aligned}
& \left|\check{a}_{q}\right|+\left|\check{b}_{q}\right| \leq c, \quad\left|\check{a}_{q}-\check{a}_{q+1}\right|+\left|\check{b}_{q}-\check{b}_{q+1}\right| \leq \frac{c}{2^{q}} \\
& \left|\check{a}_{q}\left(\rho^{\prime}, \nu\right)-\check{a}_{q}(\rho, \nu)\right|+\left|\check{b}_{q}\left(\rho^{\prime}, \nu\right)-\check{b}_{q}(\rho, \nu)\right| \leq c\left|\rho^{\prime}-\rho\right| .
\end{aligned}
$$

The symbols $V_{q}(\varepsilon, \mathbf{k})$ are homogeneous functions of order 0 in $\mathbf{k} \in \mathbb{R}^{2} \backslash\{0\}$, such that

$$
V_{q}(\varepsilon, \mathbf{k})=V_{4 m}^{(0)}(\rho, \nu, \mathbf{k})+|\varepsilon|^{2 m} \breve{V}_{q}(\rho, \nu, \mathbf{k}) .
$$

Moreover, they are smooth in $\mathbf{k}$ and for any $\gamma \geq 0$,

$$
\begin{aligned}
& \left|\partial_{\mathbf{k}}^{\gamma} V_{q}(\varepsilon, \mathbf{k})\right| \leq c|\mathbf{k}|^{-\gamma}|\varepsilon|^{2}, \\
& \left|\partial_{\mathbf{k}}^{\gamma} \breve{V}_{q}\left(\rho^{\prime}, \nu, \mathbf{k}\right)-\partial_{\mathbf{k}}^{\gamma} \breve{V}_{q}(\rho, \nu, \mathbf{k})\right| \leq c|\mathbf{k}|^{-\gamma}\left|\rho^{\prime}-\rho\right|, \\
& \left|\breve{V}_{q+1}(\rho, \nu, \mathbf{k})-\breve{V}_{q}(\rho, \nu, \mathbf{k})\right| \leq \frac{c}{2^{q}}
\end{aligned}
$$

where $c$ does not depend on $\mathbf{k}$ and $q$.

Resolvent estimates for $\varepsilon=0$ We denote by $\mathfrak{L}_{c}$ and $\mathcal{D}_{c}$ the operators $\mathfrak{L}$ and $\mathcal{D}$ corresponding to parameters $\nu=\nu_{c}, \rho=\rho_{c}$ and $\mathbb{A}=\mathbb{A}_{c}$. Recall that critical parameters $\rho_{c}=\lambda$ and $\nu_{c}$ are determined by Lemmas 2 and 5 and can be represented as functions of the lattice parameter $\tau$,

$$
\rho_{c}=\lambda=\left(1+\tau_{2}^{2}\right)^{1 / 2}\left(1+\tau_{1}^{2}\right)^{-1 / 2}, \quad \nu_{c}=\left(1+\tau_{1}^{2}\right)\left(1+\tau_{2}^{2}\right)^{-1 / 2}\left(\tau_{1}+\tau_{2}\right)^{-1} .
$$

Our task is to investigate the behavior of operators $\mathfrak{L}_{c}$ and $\mathcal{D}_{c}$ as functions of $\boldsymbol{\tau}$. In order to formulate the results we recall the following elementary property from the theory of Diophantine approximations.

lemma131 Lemma 15. For any $\alpha>0$ and $N>0$ there is a set $\mathcal{E}_{N} \subset[-N, N]$ of full measure so that for all $\rho \in \mathcal{E}_{N}$ and $\mathbf{k} \in \mathbb{Z}^{2} \backslash\{0\}$, there exists $c_{N}>0$ such that

$$
\left|k_{1}+\rho k_{2}\right| \geq c_{N}|\mathbf{k}|^{-1-\alpha} .
$$

Remark 10 The above diophantine property is compatible with Hypothesis (H.2) made in previous section (with $\alpha=1 / 3$ ).

Let $\mathcal{S}_{\text {triv }}$ be the set $\{\mathbf{k} \in\{(0,0),( \pm 1,0),(0, \pm 1)\}\}$, then the first result of this section is the following

Theorem 10 Let $\alpha>0$. Then there is a set $\mathcal{T} \subset\left(\mathbb{R}^{+}\right)^{2}$ with the following properties:

(a) $\mathcal{T}$ is a set of full measure in $\left(\mathbb{R}^{+}\right)^{2}$. 
(b) For any $\boldsymbol{\tau} \in \mathcal{T}, \lambda=\rho_{c}(\boldsymbol{\tau}), \nu=\nu_{c}(\boldsymbol{\tau})$, and $\mathbb{A}=\mathbb{A}_{c}(\boldsymbol{\tau})$, the kernel of the operator $\mathfrak{L}$ is a four dimensional space with the orthogonal basis

$$
(2 \pi)^{-1} \exp ( \pm i \mathbf{k} \cdot Y), \quad \mathbf{k} \in\{( \pm 1,0),(0, \pm 1)\} .
$$

(c) For any $\boldsymbol{\tau} \in \mathcal{T}$, there is $N>0$ so that $\lambda=\rho_{c}(\boldsymbol{\tau}) \in \mathcal{E}_{N}$. Moreover, $\rho_{c}(\boldsymbol{\tau})$ is a Lebesgue point of this set.

(d) For any $\boldsymbol{\tau} \in \mathcal{T}$, there is $c>0$, depending only on $\boldsymbol{\tau}$ and $\alpha$, such that for all $\mathbf{k} \in \mathbb{Z}^{2} \backslash \mathcal{S}_{\text {triv }}$,

$$
\left|L_{c}(\mathbf{k})\right| \geq c|\mathbf{k}|^{-1 / 2-\alpha} .
$$

Proof The proof is based on the following auxiliary lemmas.

lemma13.3 Lemma 16 The mapping $\boldsymbol{\tau} \mapsto\left(\lambda(\boldsymbol{\tau}), \nu_{c}(\boldsymbol{\tau})\right)$ takes diffeomorphically the quadrant $\left\{\tau_{i}>0\right\}$ onto the domain $\varpi$ (see Figure 2) which consists of all positive pairs $\left(\lambda, \nu_{c}\right)$ satisfying the inequalities

$$
0<\frac{1}{2 \lambda \nu_{c}}+\frac{1}{2} \lambda \nu_{c}\left(1-\lambda^{2}\right)<1, \quad 0<\frac{1}{\lambda^{2} \nu_{c}^{2}}-\left(1-\lambda^{2}\right) .
$$

The inverse mapping is defined by

$$
\begin{gathered}
\tau_{1}=\left(1-z^{2}\right)^{-1 / 2} z, \quad \tau_{2}=\left(1-z^{2}\right)^{-1 / 2}\left(z^{2}+\lambda^{2}-1\right)^{1 / 2}, \\
z=\frac{1}{2}\left[\left(\lambda \nu_{c}\right)^{-1}+\lambda \nu_{c}\left(1-\lambda^{2}\right)\right] .
\end{gathered}
$$

In particular, the symbol $L_{c}$ of the operator $\mathfrak{L}_{c}$ is a function of $\lambda, \nu_{c}$ and $\mathbf{k}$ given by the formula

$$
\frac{1}{\nu_{c}} L_{c}\left(\lambda, \nu_{c}, \mathbf{k}\right)=-\left(\lambda k_{2}+k_{1}\right)^{2}+\left[\left(\lambda k_{2}+k_{1}\right)\left(\lambda^{3} k_{2}+k_{1}\right)-\frac{1}{\lambda \nu_{c}^{2}} k_{1} k_{2}\right]^{1 / 2}
$$

Proof Substituting relations (10.15) into expression (10.11) for $\nu_{c}$ we obtain

$$
\sqrt{z^{2}+\left(\lambda^{2}-1\right)}=\left(\lambda \nu_{c}\right)^{-1}-z .
$$

Note that any solution of this equation satisfies the inequality $z<1 /(\lambda \nu)$. Next we square both sides of (10.18) to obtain (10.16).

Obviously $z$ in (10.16) satisfies (10.18) and inequalities $0<z<1$ if and only if $\lambda, \nu_{c}$ are positive and satisfy inequalities (10.14). Recalling (10.7) we get (10.17) and the lemma follows. The set $\varpi$ is the domain bounded by the curves indicated in Figure 2.

lemma13.4 Lemma 17 For any $\alpha>0$ and $\sigma>1$, there exist $N>0$ and a full measure set $\beth \subset\left[\sigma^{-1}, \sigma\right]^{2}$ with the following property. Whenever $\left(\lambda, \nu_{c}\right) \in \beth$, the inequality

$$
\left|L_{c}\left(\lambda, \nu_{c}, \mathbf{k}\right)\right| \geq c|\mathbf{k}|^{-\alpha-1 / 2}
$$

holds true for all $\mathbf{k} \in \mathbb{Z}^{2}$ with $\left|k_{i}\right|>N$. Here the positive constant $c$ depends only on $\alpha, \sigma$, and $\left(\lambda, \nu_{c}\right)$. 


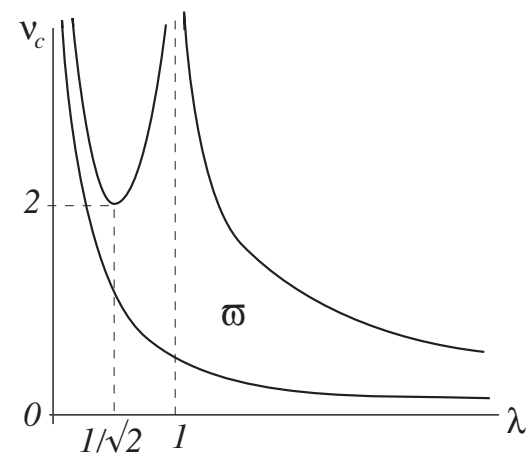

Fig. 2 domain $\varpi$

domParam

Proof The proof is given in Appendix F.

We are now in a position to complete the proof of Theorem 10. First we prove assertion (b). It suffices to prove that for almost every positive $\left(\lambda, \nu_{c}\right)$, satisfying inequalities $(10.14)$, the dispersion equation $L_{c}\left(\lambda, \nu_{c}, \mathbf{k}\right)=0$ has only five integer solutions $(0,0),( \pm 1,0)$, and $(0, \pm 1)$. It follows from formula (10.17) that this equation can be written in the equivalent form

$$
-\left(\lambda k_{2}+k_{1}\right)^{2}+\left[\left(\lambda k_{2}+k_{1}\right)\left(\lambda^{3} k_{2}+k_{1}\right)-\frac{1}{\lambda \nu_{c}^{2}} k_{1} k_{2}\right]^{1 / 2}=0 .
$$

Obviously it has the trivial solutions which form the set $\mathcal{S}_{\text {triv }}$. Let us show that for a. e. positive $\left(\lambda, \nu_{c}\right)$, satisfying (10.14), equation (10.19) has only trivial solutions. For each $\mathbf{k} \in \mathbb{Z}^{2}$ denote by $\mathcal{O}_{\mathbf{k}}$ the set of all admissible $\left(\lambda, \nu_{c}\right)$ so that $\mathbf{k}$ is solution of (10.19). In this notation, the set of all points $\left(\lambda, \nu_{c}\right) \in \varpi$, for which (10.19) has a nontrivial solution is the union

$$
\mathcal{O}=\bigcup_{\mathbf{k} \in \mathbb{Z}^{2} \backslash S_{\text {triv }}} \mathcal{O}_{\mathbf{k}}
$$

Assume, contrary to our claim, that $\mathcal{O}$ is a set of positive measure. Then there exists $\mathbf{k}^{(0)} \in \mathbb{Z}^{2} \backslash \mathcal{S}_{\text {triv }}$ so that the set $\mathcal{O}_{\mathbf{k}^{(0)}}$ has a positive measure. Hence the analytic function $L_{c}\left(\lambda, \nu, \mathbf{k}^{(0)}\right)$ vanishes on a set of positive measure, and $L_{c}\left(\lambda, \nu, \mathbf{k}^{(0)}\right)=0$ on the set $\varpi$. In particular, we have on this set $\partial_{\nu_{c}} L_{c}\left(\lambda, \nu_{c}, \mathbf{k}^{(0)}\right)=0$, hence

$$
\left[\left(\lambda k_{2}^{(0)}+k_{1}^{(0)}\right)\left(\lambda^{3} k_{2}^{(0)}+k_{1}^{(0)}\right)-\frac{1}{\lambda \nu_{c}^{2}} k_{1}^{(0)} k_{2}^{(0)}\right]^{-1 / 2} k_{1}^{(0)} k_{2}^{(0)}=0
$$

Note that, by virtue of Lemma 16, the form in the square brackets is positive on $\mathbb{Z}^{2} \backslash\{0\}$. Hence we have $k_{1}^{(0)} k_{2}^{(0)}=0$ which along with the equation $L_{c}\left(\lambda, \nu, \mathbf{k}^{(0)}\right)=0$ yields $\mathbf{k}^{(0)} \in \mathcal{S}_{\text {triv }}$. This proves (b).

Next notice that the set $\mathcal{E}=\cup_{N} \mathcal{E}_{N}$, where $\mathcal{E}_{N}$ is defined by Lemma 15 , is a set of full measure in $\mathbb{R}^{+}$. Without loss of generality we can assume that $\mathcal{E}$ 
consists only of Lebesgue points. Therefore the set $\left(\mathcal{E} \times \mathbb{R}^{+}\right) \cap(\varpi \backslash \mathcal{O})$ is a set of full measure in $\varpi$. Since the mapping $\left(\lambda, \nu_{c}\right) \rightarrow \boldsymbol{\tau}$ defined by (10.15) is a diffeomorphism, the set

$$
\tilde{\mathcal{I}}=\boldsymbol{\tau}\left(\left(\mathcal{E} \times \mathbb{R}^{+}\right) \cap(\varpi \backslash \mathcal{O})\right)
$$

is a set of full measure in $\mathbb{R}^{+2}$, which proves (c).

It remains to prove $(\mathrm{d})$. For each $l \geq 1$ denote by $\mathcal{I}_{l}$ the square $\left[l^{-1}, l\right]^{2} \subset \mathbb{R}^{+2}$. Further the notation $\Upsilon(N)$ stands for the set of all points $\mathbf{k} \in \mathbb{Z}^{2}$ satisfying the inequalities $\left|k_{i}\right| \geq N, i=1,2$. Its complement $\mathbb{Z}^{2} \backslash \Upsilon(N)$ is the cross obtained by union of the strips $\left\{\left|k_{i}\right|<N\right\}$.

It follows from Lemma 16 that the mapping $\boldsymbol{\tau} \mapsto(\lambda(\boldsymbol{\tau}), \nu(\boldsymbol{\tau}))$ takes diffeomorphically the square $\mathcal{I}_{l}$ onto a compact set which belongs to some square $\left[\sigma_{l}^{-1}, \sigma_{l}\right]^{2}$. Next applying Lemma 17 we conclude that there exists a number $N_{l}>0$ and a set of full measure $\beth_{l} \subset\left[\sigma_{l}^{-1}, \sigma_{l}\right]^{2}$ with the following property: whenever $\left(\lambda, \nu_{c}\right) \in \beth_{l}$, we have

$$
\left|L_{c}\left(\lambda, \nu_{c}, \mathbf{k}\right)\right| \geq c\left(\lambda, \nu_{c}, l\right)|\mathbf{k}|^{-1 / 2-\alpha} \text { for all } \mathbf{k} \in \Upsilon\left(N_{l}\right) .
$$

On the other hand, we have

$$
\left|L_{c}\left(\lambda, \nu_{c}, \mathbf{k}\right)\right| \rightarrow \infty \text { as }|\mathbf{k}| \rightarrow \infty, \mathbf{k} \in \mathbb{Z}^{2} \backslash \Upsilon\left(N_{l}\right),
$$

uniformly with respect to $\left(\lambda, \nu_{c}\right) \in \beth_{l}$. Hence we can choose $N_{l}$ so large that

$$
\left|L_{c}\left(\lambda, \nu_{c}, \mathbf{k}\right)\right| \geq c\left(\lambda, \nu_{c}, l\right)|\mathbf{k}|^{-1 / 2-\alpha} \text { for all }|\mathbf{k}| \geq N_{l},
$$

uniformly in $\left(\lambda, \nu_{c}\right) \in \beth_{l}$. Since the set of all $\mathbf{k}$ in the disc $D=\left\{|\mathbf{k}| \leq N_{l}\right\}$ is finite, there exists a constant $c\left(\lambda, \nu_{c}, l\right)$ such that for any $l>3$ and $\left(\lambda, \nu_{c}\right) \in$ $\beth_{l} \cap\left(\mathcal{E} \times \mathbb{R}^{+}\right) \cap(\varpi \backslash \mathcal{O})$ inequality $(10.21)$ holds true for all $\mathbf{k} \in \mathbb{Z}^{2} \backslash \mathcal{S}_{\text {triv }}$.

Denote by $\mathcal{F}_{l}$ the set of all $\tau \in \mathcal{I}_{l} \subset \mathbb{R}^{+2}$ which consists of all points $\boldsymbol{\tau}$ such that $(\lambda(\boldsymbol{\tau}), \nu(\boldsymbol{\tau})) \in \beth_{l}$. Obviously inequality (10.21) holds true for all $(\lambda(\boldsymbol{\tau}), \nu(\boldsymbol{\tau}))$ with $\boldsymbol{\tau} \in \mathcal{F}_{l} \cap \tilde{\mathcal{I}}$. Since the mapping $\boldsymbol{\tau} \rightarrow(\lambda(\boldsymbol{\tau}), \nu(\boldsymbol{\tau}))$ is a diffeomorphism and $\beth_{l}$ is a set of full measure in $\left[\sigma_{l}^{-1}, \sigma_{l}\right]^{2}$, the set $\mathcal{F}_{l}$ is a set of full measure in $\mathcal{I}_{l}$. Therefore, the set

$$
\mathcal{T}=\bigcup_{l \geq 3} \mathcal{F}_{l} \cap \tilde{\mathcal{I}}
$$

meets all requirements of Theorem 10, which completes the proof.

Resolvent estimates for $\varepsilon \neq 0$. Now we consider the general case when the parameters $\rho, \nu$, elements of the matrices $\mathbb{A}_{q}$ and symbols $V_{q}$ depend on the small parameter $\varepsilon=\left(\varepsilon_{1}, \varepsilon_{2}\right)$. The following theorem is the main result of this section.

thds0 Theorem 11 Let $\boldsymbol{\tau} \in \mathcal{T}$, and $0<\alpha<1 / 2$, and assume that condition (6.4) holds true. Then, there exists $\epsilon_{0}>0$, such that for any eligible $\nu$ for having non empty $(\rho, \nu) \in \omega_{\epsilon_{0}}$, there exists a set $\mathcal{E}^{\prime}(\nu)$ with $\mathcal{E}^{\prime}(\nu) \times\{\nu\} \subset \omega_{\epsilon_{0}}$ with the following property. 
(a) The set $\mathcal{E}^{\prime}(\nu)$ is dense at $\lambda$, meas $\left(\left.\omega_{\epsilon}\right|_{\nu}\right)^{-1}$ meas $\mathcal{E}^{\prime}(\nu) \cap\{|\lambda-\rho| \leq \epsilon\} \rightarrow 1$ as $\epsilon \rightarrow 0$, uniformly in $\nu$.

(b) If $\rho \in \mathcal{E}^{\prime}(\nu)$, then for any $\mathbf{k} \in \mathbb{Z}^{2} \backslash S_{\text {triv }}$,

$$
\begin{gathered}
\left|\rho k_{2}+k_{1}\right| \geq c_{0}|\mathbf{k}|^{-\alpha-1} \\
\left|\nu\left(\rho k_{2}+k_{1}\right)^{2}-\left(\mathbb{A}_{q} \widetilde{\mathbf{k}} \cdot \widetilde{\mathbf{k}}\right)^{1 / 2}-V_{q}(\varepsilon, \mathbf{k})\right| \geq c_{0}|\mathbf{k}|^{-\alpha-1 / 2} .
\end{gathered}
$$

Proof The proof imitates the proof of assertion (d) in Theorem 10 and is based on the following proposition, which proof is given in Appendix F.

Proposition 5 Under the assumptions of Theorem 11, there exist $N>0$ and a set $\mathcal{E}_{N}^{\prime}(\nu)$ such that $\mathcal{E}_{N}^{\prime}(\nu)$ satisfies the condition (10.22). Furthermore, if $\rho \in \mathcal{E}_{N}^{\prime}(\nu)$, then inequality (10.24) holds true for any $\mathbf{k} \in \mathbb{Z}^{2}$ with $\left|k_{i}\right| \geq N$.

Let us turn to the proof of Theorem 11. By virtue of Proposition 5, whenever $\rho \in \mathcal{E}_{N}^{\prime}(\nu)$, inequality (10.24) holds true for all $\mathbf{k} \in \Upsilon(N)$, where the notation $\Upsilon(N)$ stands for the set of all points $\mathbf{k}$ satisfying $\left|k_{i}\right| \geq N$. On the other hand, we have

$$
\left|\nu\left(\rho k_{2}+k_{1}\right)^{2}-\left(\mathbb{A}_{q} \widetilde{\mathbf{k}} \cdot \widetilde{\mathbf{k}}\right)^{1 / 2}-V_{q}(\varepsilon, \mathbf{k})\right| \rightarrow \infty \text { as }|\mathbf{k}| \rightarrow \infty, \mathbf{k} \in \mathbb{Z}^{2} \backslash \Upsilon(N),
$$

uniformly in $(\rho, \nu) \in \omega_{\epsilon_{0}}$. It follows from this that we can choose $N$ so large such for all $|\mathbf{k}| \geq N$,

$$
\left|\nu\left(\rho k_{2}+k_{1}\right)^{2}-\left(\mathbb{A}_{q} \tilde{\mathbf{k}} \cdot \widetilde{\mathbf{k}}\right)^{1 / 2}-V_{q}(\varepsilon, \mathbf{k})\right| \geq c_{0}|\mathbf{k}|^{-1 / 2-\alpha}
$$

uniformly in $\rho \in \mathcal{E}_{N}^{\prime}(\nu)$. Next note that by choice of $\boldsymbol{\tau}$, we have for all $\mathbf{k} \in \mathbb{Z}^{2}$,

$$
c(\boldsymbol{\tau})^{-1}|\mathbf{k}|^{2} \leq \mathbb{A}_{c} \tilde{\mathbf{k}} \cdot \widetilde{\mathbf{k}} \leq c(\boldsymbol{\tau})|\mathbf{k}|^{2}
$$

where the positive constant $c$ depends only on the choice of $\tau \in \mathcal{T}$. Since the set of all $\mathbf{k}$ in the $\operatorname{disc} D=\{|\mathbf{k}| \leq N\}$ is finite, it follows from this and Lemma 14 that for all $\mathbf{k} \in D \backslash \mathcal{S}_{\text {triv }}$,

$$
\left|\left(\mathbb{A}_{q} \widetilde{\mathbf{k}} \cdot \widetilde{\mathbf{k}}\right)^{1 / 2}-\left(\mathbb{A}_{c} \widetilde{\mathbf{k}} \cdot \widetilde{\mathbf{k}}\right)^{1 / 2}\right| \leq C(N, \boldsymbol{\tau})|\varepsilon|^{2} .
$$

From this we conclude that for every such $\mathbf{k}$,

$$
\left|\nu\left(\rho k_{2}+k_{1}\right)^{2}-\left(\mathbb{A}_{q} \widetilde{\mathbf{k}} \cdot \widetilde{\mathbf{k}}\right)^{1 / 2}-V_{q}(\varepsilon, \mathbf{k})+L_{c}\left(\rho_{c}, \nu_{c}, \mathbf{k}\right)\right| \leq C(N, \tau)|\varepsilon|^{2} .
$$

Since $\tau \in \mathcal{T}$, it follows from this and estimate (10.13) in Theorem 10 that

$$
\begin{aligned}
& \inf _{D \backslash \mathcal{S}_{\text {triv }}}\left|\nu\left(\rho k_{2}+k_{1}\right)^{2}-\left(\mathbb{A}_{q} \widetilde{\mathbf{k}} \cdot \widetilde{\mathbf{k}}\right)^{1 / 2}-V_{q}(\boldsymbol{\varepsilon}, \mathbf{k})\right| \geq \\
& c_{0}(\tau) N^{-1 / 2-\alpha}-C(N, \tau)|\boldsymbol{\varepsilon}|^{2} .
\end{aligned}
$$

Hence there is $\epsilon_{0}>0$, depending only on $\tau, N$ and the constant $c$ in Lemma 14 so that the inequality

$$
\left|\nu\left(\rho k_{2}+k_{1}\right)^{2}-\left(\mathbb{A}_{q} \widetilde{\mathbf{k}} \cdot \widetilde{\mathbf{k}}\right)^{1 / 2}-V_{q}(\boldsymbol{\varepsilon}, \mathbf{k})\right| \geq \frac{1}{2} c_{0}(\tau) N^{-1 / 2-\alpha}
$$

holds true for all $\mathbf{k} \in D \backslash \mathcal{S}_{\text {triv }}$ and eligible $(\rho, \nu) \in \omega_{\epsilon_{0}}$. It remains to note that by virtue of $(10.25)$ the set $\mathcal{E}^{\prime}(\nu)=\left.\mathcal{E} \cap \mathcal{E}_{N}^{\prime}(\nu) \cap \omega_{\epsilon_{0}}\right|_{\nu}$ meets all requirements of Theorem 11. 


\section{Inversion of the differential of operator $\widetilde{\mathcal{F}}$}

In this section we complete the inversion of the approximate differential given by the system (7.17), (7.18), (7.19). First we invert the scalar equation (7.29) in using the results of Theorems 8 and 11. For this we need to compute 4 scalar coefficients, which are analytic functions of $\boldsymbol{\tau}$, allowing to solve the reduced linear system in ker $\mathfrak{L}_{c}$. Once a simple non degeneracy condition is verified, which holds for $\boldsymbol{\tau}$ in a full measure set of $\mathbb{R}^{+2}$, the equation (7.29) is inverted, and it remains to solve the two-component equation (7.28) with respect to $v$ (which has a zero average), and solve (7.18) with respect to $\varsigma$.

Inversion of the operator in normal form. Here we solve the linear equation

$$
\Pi(\mathfrak{L}+\mathfrak{V}+\mathfrak{F}) \Pi \bar{u}+(\mathbb{I}-\Pi)(\mathfrak{L}+\mathfrak{P}) \bar{u}=\bar{f}
$$

where $\bar{f} \in H_{o}^{s}$, and where we look for $\bar{u} \in H_{o}^{s-1}$ with $1 \leq s \leq r-25$ as assumed in Hypothesis H.1 and H.2.

Let us define the orthogonal projection $P_{0}$ on the two-dimensional subspace ker $\mathfrak{L}_{c}=\left\{\sin y_{1}, \sin y_{2}\right\}$ (notice that the 4-dimensional kernel becomes 2-dimensional once restricted to odd functions of $Y$ ). We have the following decomposition in all spaces $H_{o}^{s}$

$$
\begin{aligned}
\bar{u} & =u_{0}+u_{1}+u_{2}, \Pi \bar{u}=u_{0}+u_{1}, \\
(\mathbb{I}-\Pi) \bar{u} & =u_{2}, \quad P_{0} \bar{u}=u_{0}, \quad Q_{0} \bar{u}=u_{1} .
\end{aligned}
$$

Equation (11.1) is decomposed into the following system

$$
\begin{aligned}
\left(\mathfrak{L}+\mathfrak{V}+P_{0} \mathfrak{F}\right) u_{0}+P_{0} \mathfrak{F} u_{1} & =P_{0} \bar{f}, \\
\left(\mathfrak{L}+\mathfrak{V}+Q_{0} \mathfrak{F}\right) u_{1}+Q_{0} \mathfrak{F} u_{0} & =Q_{0} \bar{f}, \\
(\mathbb{I}-\Pi)(\mathfrak{L}+\mathfrak{P}) u_{2}+(\mathbb{I}-\Pi) \mathfrak{P}\left(u_{0}+u_{1}\right) & =(\mathbb{I}-\Pi) \bar{f} .
\end{aligned}
$$

$$
\begin{aligned}
& \text { equKerL_c } \\
& \hline \hline \text { equQ_0 } \\
& \hline \hline \text { equ1-Pi } \\
& \hline
\end{aligned}
$$

By using Theorem 11 and (8.27) we solve (11.3), (11.4) with respect to $\left(u_{1}, u_{2}\right)$ in function of $u_{0}$ and $\bar{f}$, as

$$
\begin{aligned}
& u_{1}=\left(\mathbb{I}+(\mathfrak{L}+\mathfrak{V})^{-1} Q_{0} \mathfrak{F}\right)^{-1}(\mathfrak{L}+\mathfrak{V})^{-1}\left\{-Q_{0} \mathfrak{F} u_{0}+Q_{0} \bar{f}\right\}, \\
& u_{2}=\left[\mathbb{I}+((\mathbb{I}-\Pi) \mathfrak{L})^{-1} \mathfrak{P}\right]^{-1}((\mathbb{I}-\Pi) \mathfrak{L})^{-1}\left\{(\mathbb{I}-\Pi) \bar{f}-(\mathbb{I}-\Pi) \mathfrak{P}\left(u_{0}+u_{1}\right)\right\} .
\end{aligned}
$$

We observe that the linear operator $\left(\mathbb{I}+(\mathfrak{L}+\mathfrak{V})^{-1} Q_{0} \mathfrak{F}\right)$ has a bounded inverse in $H_{o}^{s-1}$ and $\left[\mathbb{I}+((\mathbb{I}-\Pi) \mathfrak{L})^{-1} \mathfrak{P}\right]$ has a bounded inverse in $H_{o}^{s}$, and since $u_{0}$ is in $H_{o}^{p}$ for any $p$, we then obtain the estimates

$$
\begin{array}{r}
\left\|u_{1}\right\|_{s-1} \leq c\left\|Q_{0} \bar{f}\right\|_{s}+c \epsilon^{1 / 2}\left\|u_{0}\right\| \\
\left\|u_{1}\right\|_{s-1+l} \leq c\left\|Q_{0} \bar{f}\right\|_{s+l}+c E_{l}\left(\left\|Q_{0} \bar{f}\right\|_{s}+\left\|u_{0}\right\|\right),
\end{array}
$$

and

$$
\begin{array}{r}
\left\|u_{2}\right\|_{s} \leq c\|(\mathbb{I}-\Pi) \bar{f}\|_{s-1}+c \epsilon^{1 / 2}\left(\left\|u_{0}\right\|+\left\|u_{1}\right\|_{s-1}\right), \\
\left\|u_{2}\right\|_{s+l} \leq c\|(\mathbb{I}-\Pi) \bar{f}\|_{s-1+l}+c E_{l}\left(\|(\mathbb{I}-\Pi) \bar{f}\|_{s-1}+\left\|u_{0}\right\|+\left\|u_{1}\right\|_{s-1}\right),
\end{array}
$$


where $E_{l}$ is determined by (9.22) and denotes $C^{l+r}$ norm of functions $U$ and $X$. This leads to

$$
\begin{gathered}
\left\|u_{2}\right\|_{s} \leq c\left(\|(\mathbb{I}-\Pi) \bar{f}\|_{s-1}+\left\|Q_{0} \bar{f}\right\|_{s}\right)+c \epsilon^{1 / 2}\left\|u_{0}\right\|, \\
\left\|u_{2}\right\|_{s+l} \leq c\left(\|(\mathbb{I}-\Pi) \bar{f}\|_{s-1+l}+\left\|Q_{0} \bar{f}\right\|_{s+l}\right)+ \\
c E_{l}\left(\|(\mathbb{I}-\Pi) \bar{f}\|_{s-1}+\left\|Q_{0} \bar{f}\right\|_{s}+\left\|u_{0}\right\|\right) .
\end{gathered}
$$

Now replacing $u_{1}$ by (11.5) in (11.2), we obtain the following two-dimensional linear equation

$$
\begin{aligned}
& \left(\mathfrak{L}+\mathfrak{V}+P_{0} \mathfrak{F}\right) u_{0}-P_{0} \mathfrak{F}\left[\mathbb{I}+(\mathfrak{L}+\mathfrak{V})^{-1} Q_{0} \mathfrak{F}\right]^{-1}(\mathfrak{L}+\mathfrak{V})^{-1} Q_{0} \mathfrak{F} u_{0} \\
= & P_{0} \bar{f}-P_{0} \mathfrak{F}\left(\mathbb{I}+(\mathfrak{L}+\mathfrak{V})^{-1} Q_{0} \mathfrak{F}\right)^{-1}(\mathfrak{L}+\mathfrak{V})^{-1} Q_{0} \bar{f} .
\end{aligned}
$$

Our aim is now to study the structure of the left hand side of this twodimensional system.

Since $\mathfrak{L}_{c} u_{0}=0$, and recalling that $O(\epsilon) \equiv O\left(\varepsilon_{1}^{2}+\varepsilon_{2}^{2}\right)$, we have by construction, $\left\|(\mathfrak{L}+\mathfrak{V}) u_{0}\right\| \leq c \epsilon\left\|u_{0}\right\|$. Moreover, we have the estimate (9.20) for $\mathfrak{F} u$ which shows that

$$
\left\|P_{0} \mathfrak{F}\left[\mathbb{I}+(\mathfrak{L}+\mathfrak{V})^{-1} Q_{0} \mathfrak{F}\right]^{-1}(\mathfrak{L}+\mathfrak{V})^{-1} Q_{0} \mathfrak{F} u_{0}\right\| \leq c \epsilon\left\|u_{0}\right\| .
$$

To show that $P_{0} \mathfrak{F} u_{0}$ is also of order $\epsilon|| u_{0} \|$, we use the fact that the terms of or$\operatorname{der} \epsilon^{1 / 2}$ in the operator $\mathfrak{F}$ only originate from the first harmonics $e^{ \pm i y_{1}}, e^{ \pm i y_{2}}$ appearing in the first order terms in $\left(\varepsilon_{1}, \varepsilon_{2}\right)$ of $U$. Let us now decompose $u_{0}$ in the basis of ker $\mathfrak{L}_{c}$ as

$$
u_{0}=\gamma_{1} \sin y_{1}+\gamma_{2} \sin y_{2},
$$

then in $\mathfrak{F} u_{0}$ at order 1 in $\left(\varepsilon_{1}, \varepsilon_{2}\right)$ we only have second harmonics. It then results that the projection $P_{0}$ cancels these terms. This proves that the lowest order terms on the left hand side of (11.8) are of order $\epsilon\left\|u_{0}\right\|$. For identifying the $2 \times 2$ matrix of order $\epsilon$ giving the principal part of equation (11.8), let us introduce the formal expansion in powers of $\varepsilon_{1}, \varepsilon_{2}$ of the linear operator occuring in (11.1):

$$
\Pi(\mathfrak{L}+\mathfrak{V}+\mathfrak{F}) \Pi+(\mathbb{I}-\Pi)(\mathfrak{L}+\mathfrak{P})=\mathfrak{L}_{c}+\mathfrak{L}^{(1)}+\mathfrak{L}^{(2)}+\mathfrak{L}^{(3)}
$$

where

$$
\mathfrak{L}^{(1)}=\varepsilon_{1} \mathfrak{L}^{(1,0)}+\varepsilon_{2} \mathfrak{L}^{(0,1)}, \quad \mathfrak{L}^{(2)}=\varepsilon_{1}^{2} \mathfrak{L}^{(2,0)}+\varepsilon_{1} \varepsilon_{2} \mathfrak{L}^{(1,1)}+\varepsilon_{2}^{2} \mathfrak{L}^{(0,2)},
$$

and $\mathfrak{L}^{(3)}=O\left(|\boldsymbol{\varepsilon}|^{3}\right)$. Here operators $\mathfrak{L}^{(i, j)}$ are independent of $\boldsymbol{\varepsilon}$. Then we have the following

Lemma 18 The lowest order terms on the left hand side of the two-dimensional equation (11.8) have the form

$$
\left(P_{0} \mathfrak{L}^{(2)} P_{0}-P_{0} \mathfrak{L}^{(1)} \mathfrak{L}_{c}^{-1} \mathfrak{L}^{(1)} P_{0}\right) u_{0} .
$$

With the representation (11.9) of $u_{0}$, the two-components of these terms take the form $\widetilde{a}_{1} \varepsilon_{1}^{2} \gamma_{1}+\widetilde{b}_{1} \varepsilon_{1} \varepsilon_{2} \gamma_{2}$ and $\widetilde{a}_{2} \varepsilon_{1} \varepsilon_{2} \gamma_{1}+\widetilde{b}_{2} \varepsilon_{2}^{2} \gamma_{2}$, where coefficients $\widetilde{a}_{j}, \widetilde{b}_{j}$ 
are analytic functions of $\boldsymbol{\tau}=\left(\tau_{1}, \tau_{2}\right)$ given explicitly by (G.18) in Appendix G. Moreover, if (6.4), and

$$
\widetilde{a}_{1} \widetilde{b}_{2}-\widetilde{a}_{2} \widetilde{b}_{1} \neq 0
$$

hold, and $\delta<\varepsilon_{1} / \varepsilon_{2}<1 / \delta$, where $\delta$ is arbitrary such that $0<\delta<1$, we have a unique solution $u_{0}$ in $\operatorname{ker} \mathfrak{L}_{c}$ of (11.8), with the estimate

$$
\left\|u_{0}\right\| \leq \frac{c}{\epsilon}\|\bar{f}\|_{s}
$$

Proof We have formally

$$
\begin{aligned}
P_{0}\left(\mathfrak{L}^{(1)}+\mathfrak{L}^{(2)}+\mathfrak{L}^{(3)}\right)\left(u_{0}+u_{1}+u_{2}\right) & =P_{0} \bar{f}, \\
\mathfrak{L}_{c}\left(u_{1}+u_{2}\right)+\left(\mathfrak{L}^{(1)}+\mathfrak{L}^{(2)}+\mathfrak{L}^{(3)}\right)\left(u_{0}+u_{1}+u_{2}\right) & =\left(\mathbb{I}-P_{0}\right) \bar{f},
\end{aligned}
$$

which leads to

$$
u_{1}+u_{2}=-\mathfrak{L}_{c}^{-1} \mathfrak{L}^{(1)} u_{0}+O\left(|\varepsilon|^{2} \| u_{0}||\right)+\left\{\mathfrak{L}_{c}^{-1}\left(\mathbb{I}-P_{0}\right)+O(|\varepsilon|)\right\}\left(\mathbb{I}-P_{0}\right) \bar{f} .
$$

We now observe that $P_{0} \mathfrak{L}^{(1)} P_{0}=0$ for the reason invoqued above (integral of harmonics of odd order), hence it is clear that the term of order $|\varepsilon|^{2}$ in the left hand side of (11.8) is given by (11.11). Due to the form (11.10) of operators $\mathfrak{L}^{(j)}$, it is clear by a simple examination of harmonics, that the two components of $\left(P_{0} \mathfrak{L}^{(2)} P_{0}-P_{0} \mathfrak{L}^{(1)} \mathfrak{L}_{c}^{-1} \mathfrak{L}^{(1)} P_{0}\right) u_{0}$ take the form

$$
\left\{\begin{array}{c}
\left(\widetilde{a}_{1} \varepsilon_{1}^{2}+a_{1}^{\prime} \varepsilon_{2}^{2}\right) \gamma_{1}+\widetilde{b}_{1} \varepsilon_{1} \varepsilon_{2} \gamma_{2} \\
\widetilde{a}_{2} \varepsilon_{1} \varepsilon_{2} \gamma_{1}+\left(\widetilde{b}_{2} \varepsilon_{2}^{2}+b_{2}^{\prime} \varepsilon_{1}^{2}\right) \gamma_{2} .
\end{array}\right.
$$

Now, the fact that $a_{1}^{\prime}=b_{2}^{\prime}=0$ results from a subtle argument resulting from the form of the bifurcation system (A.9). This is proved in Appendix $\mathrm{G}$, as well as the explicit expressions giving the coefficients $\widetilde{a}_{1}, \widetilde{a}_{2}, \widetilde{b}_{1}, \widetilde{b}_{2}$. Now the inverse of the $2 \times 2$ matrix is easily bounded by $c /|\varepsilon|^{2}$ in the region $\delta<$ $\varepsilon_{1} / \varepsilon_{2}<1 / \delta$, which ends the proof of Lemma 18 .

Let us define a new full measure set in $\mathbb{R}^{+2}$ by

$$
\widetilde{\mathcal{T}}=\mathcal{T} \cap\left\{\rho_{1} \nu_{2}-\rho_{2} \nu_{1} \neq 0\right\} \cap\left\{\widetilde{a}_{1} \widetilde{b}_{2}-\widetilde{a}_{2} \widetilde{b}_{1} \neq 0\right\} .
$$

Then, collecting the estimates (11.6), (11.7), and the result of Lemma 18 we have now the following

Theorem 12 Assume that Hypothesis $\boldsymbol{H} . \mathbf{1}$ and $\boldsymbol{H} .2$ hold, that $\boldsymbol{\tau} \in \widetilde{\mathcal{T}}, \rho \in$ $\mathcal{E}^{\prime}(\nu)$ with $\alpha=1 / 3$, and $\bar{f} \in H_{o}^{s}$, with $1 \leq s \leq r-25$, and assume that $\delta<\varepsilon_{1} / \varepsilon_{2}<1 / \delta, 0<\delta<1$ being fixed and $E_{l}$ determined by (9.22), then the linear equation (11.1) has a unique solution $\bar{u} \in H_{o}^{s-1}$ with the following estimate

$$
\|\bar{u}\|_{s-1} \leq \frac{c}{\epsilon}\|\bar{f}\|_{s}, \quad\|\bar{u}\|_{s+l-1} \leq \frac{c}{\epsilon}\left(\|\bar{f}\|_{s+l}+E_{l}\|\bar{f}\|_{s}\right)
$$

where $c$ only depends on $\boldsymbol{\tau}, r, \delta$ and $s, l$. 
Inversion of system (7.17)-(7.19). We consider here the full system (7.17)(7.19), and we prove the following

invLinOp Theorem 13 Assume that Hypothesis $\boldsymbol{H} .1$ and $\boldsymbol{H} .2$ hold with $9 \leq s \leq$ $r-25$, that $\boldsymbol{\tau} \in \widetilde{\mathcal{T}}, \rho \in \mathcal{E}^{\prime}(\nu)$ with $\alpha=1 / 3$, and assume that $\delta<\varepsilon_{1} / \varepsilon_{2}<1 / \delta$, $0<\delta<1$ being fixed and. Then for any

$$
(f, g, \mathbf{h}) \in H_{o}^{s+l} \times H_{e}^{s+l} \times\left(H_{e}^{s+l}\right)^{2},
$$

there is a unique solution $(u, \varsigma, v, \delta \mu, \delta \varpi) \in H_{o}^{s+l-2} \times H_{e}^{s+l-3} \times\left(H_{o}^{s+l-9}\right)^{2} \times$ $\mathbb{R}^{2}$ of the linear system (7.17)-(7.19) where $\delta \mathbf{u}=\mathbf{u}^{\perp} \delta \varpi$, and the following estimates hold

$$
\begin{gathered}
\|u\|_{s-2}+\|v\|_{s-9} \mid+\|\varsigma\|_{s-3} \leq c(\epsilon)^{-1}\|f, g, \mathbf{h}\|_{s} \\
\|u\|_{s-2+l}+\|v\|_{s-9+l} \mid+\|\varsigma\|_{s-3+l} \leq c(\epsilon)^{-1}\left(\|f, g, \mathbf{h}\|_{s+l}+E_{l}\|f, g, \mathbf{h}\|_{s}\right), \\
|\delta \mu|+\left|\delta \mathbf{u}^{\perp}\right| \leq c(\epsilon)^{-1 / 2}\left(\|f\|_{5}+\|g\|_{5}+\|\mathbf{h}\|_{3}\right) .
\end{gathered}
$$

Proof From (7.23), (7.27), the right hand side of (7.29) is in fact

$$
f+\frac{1}{H} \mathcal{D}\left(\frac{g}{\tilde{\mathfrak{a}}}\right)+g \frac{q_{1}}{\widetilde{\mathfrak{a}}}-\boldsymbol{\Xi}_{2}[0, \mathbf{G}] \mathbf{p}_{2} \cdot \mathbf{u}^{\perp}-\boldsymbol{\Xi}_{1}[0, \mathbf{G}] p_{3}=\mathfrak{S}^{-1} \bar{f},
$$

with $\mathbf{G}$ given by (7.24). After a careful examination of the 0-order (in $\epsilon$ ) part of $\mathbf{T}\left[\nabla u,-\frac{1}{H \mathfrak{a}} \mathcal{D} u\right]$ which has a zero average, we have easily

$$
\begin{gathered}
\left|\boldsymbol{\Xi}_{j}[u, \mathbf{G}]\right| \leq c\left(\|\mathbf{G}\|_{0}+\epsilon^{1 / 2}\|\mathbf{Y}[u]\|_{0}\right), \\
\|\mathbf{G}\|_{s-3} \leq c\left(\|g\|_{s}+\|\mathbf{h}\|_{s}\right),\left.\|\mathbf{Y}[u]\|\right|_{s-3} \leq c\|u\|_{s}, \\
\|\mathbf{G}\|_{s-3+l} \leq c\left(\|g\|_{s+l}+\|\mathbf{h}\|_{s+l}\right)+c E_{l}\left(\|g\|_{s}+\|\mathbf{h}\|_{s}\right), \\
\|\mathbf{Y}[u]\|_{s-3+l} \leq c\|u\|_{s+l}+c E_{l}\|u\|_{s} .
\end{gathered}
$$

Hence, from the properties (9.21) of operators $\mathfrak{S}$ and $\mathfrak{T}$ given in Theorem 8 , and from (9.27) for $\mathfrak{g}$, we have easily from Theorem 12 the estimate (11.13) for $u=\mathfrak{T} \bar{u}$. We arrive to equation (7.28) for the two components of $v$ which has a zero average by construction. The right hand side of (7.28) is bounded as

$$
\begin{aligned}
\| \mathbf{G} & -\boldsymbol{\Xi}_{1}[u, \mathbf{G}] \mathbf{Q}(1)-\boldsymbol{\Xi}_{2}[u, \mathbf{G}] \mathbf{U}\left(\mathbf{u}^{\perp}\right)-\mathbf{Y}[u] \|_{s-5} \\
& \leq c\left(\|u\|_{s-2}+\|g\|_{s-2}+\|\mathbf{h}\|_{s-2}\right) \leq \frac{c}{\epsilon}\left(\|f\|_{s}+\|g\|_{s}+\|\mathbf{h}\|_{s}\right),
\end{aligned}
$$

and accordingly

$$
\begin{aligned}
& \left\|\mathbf{G}-\boldsymbol{\Xi}_{1}[u, \mathbf{G}] \mathbf{Q}(1)-\boldsymbol{\Xi}_{2}[u, \mathbf{G}] \mathbf{U}\left(\mathbf{u}^{\perp}\right)-\mathbf{Y}[u]\right\| \|_{s-5+l} \leq \\
& \frac{c}{\epsilon}\left(\|f\|_{s+l}+\|g\|_{s+l}+\|\mathbf{h}\|_{s+l}\right)+\frac{c}{\epsilon} E_{l}\left(\|f\|_{s}+\|g\|_{s}+\|\mathbf{h}\|_{s}\right) .
\end{aligned}
$$

Now by inverting the matrix symbol of the differential operator acting on $v$, we have $2 / 3\left(k_{1}+\rho k_{2}\right)^{2}$ at the denominator, and a first order term in $\mathbf{k}$ at the numerator, hence taking into account of the assumption H.2 for $\rho$, we have a loss of 4 derivatives in inverting $\mathcal{D}-\frac{\varrho}{3}$ div. The estimate (11.13) for $v$ follows. The estimate (11.13) for $\varsigma$ follows from formula (7.20), from the estimate (11.15) of $\boldsymbol{\Xi}_{j}$ and from the estimate (11.13) for $u$. The estimates (11.14) on $\delta \mu$, and $\delta \varpi$ given by (7.25), (7.26) are obtained by using (11.15). 


\section{Nonlinear problem. Proof of Theorem 2}

In this section we complete the proof of the main Theorem 2 on the existence of asymmetrical waves of finite amplitude. To this end we exploit the general version of the Nash-Moser Implicit Function Theorem proved in Appendix $\mathrm{N}$ of [15]. This result concerns the solvability of the operator equation

$$
\Phi(W, \gamma)=0
$$

in scales of Banach spaces $E_{s}$ and $F_{s}$ parametrized by $s \in \mathbb{N}_{0}=\mathbb{N} \cup\{0\}$, and supplemented with the norms $\|\cdot\|_{s}$ and $|\cdot|_{s}$. It is supposed that they satisfy the following conditions.

(A1) For $t<s$ there exists $c(t, s)$ such that

$\|\cdot\|_{t} \leq c(t, s)\|\cdot\|_{s}, \quad|\cdot|_{t} \leq c(t, s)|\cdot|_{s}$

(A2) For $\lambda \in[0,1]$ with $\lambda t+(1-\lambda) s \in \mathbb{N}$,

$$
\|\cdot\|_{\lambda t+(1-\lambda) s} \leq c(t, s)\|\cdot\|_{t}^{\lambda}\|\cdot\|_{s}^{1-\lambda}, \quad|\cdot|_{\lambda t+(1-\lambda) s} \leq c(t, s)|\cdot|_{t}^{\lambda}|\cdot|_{s}^{1-\lambda} .
$$

(A3) There exists a family of smoothing operators $S_{\wp}$ defined over the first scale such that for $\wp>0$ and $t<s$,

$$
\begin{gathered}
\left\|S_{\wp} W\right\|_{t} \leq c(t, s)\|W\|_{s}, \quad\left\|S_{\wp} W\right\|_{s} \leq c(t, s) \wp^{t-s}\|W\|_{t}, \\
\left\|S_{\wp} W-W\right\|_{t} \leq c(t, s) \wp^{s-t}\|W\|_{s},
\end{gathered}
$$

and, if $|\gamma| \mapsto \wp(|\gamma|)$ is a smooth, increasing, convex function on $[0, \infty)$ with $\wp(0)=0$, then, for $0<\gamma_{1}<\gamma_{2}$,

$$
\left\|\left(S_{\wp\left(\gamma_{1}\right)}-S_{\wp\left(\gamma_{2}\right)}\right) W\right\|_{s} \leq c(t, s)\left|\gamma_{1}-\gamma_{2}\right| \wp^{\prime}\left(\gamma_{2}\right) \wp\left(\gamma_{1}\right)^{t-s-1}\|W\|_{t} .
$$

(B1) Operators $\Phi(\cdot, \gamma)$, depend on a small parameter $\gamma \in\left[-\gamma_{0}, \gamma_{0}\right]$, and map a neighborhood of 0 in $E_{r}$ into $F_{q}$. Suppose that there exist

$$
\sigma \leq q \leq r-1, \quad \sigma, q, r \in \mathbb{N}_{0}
$$

and, for all $l \in \mathbb{N}_{0}$, numbers $c(l)>0$ and $\gamma(l) \in\left[-\gamma_{0}, \gamma_{0}\right]$ with the following properties for all $W, U, W_{i}, U_{i} \in B$ and $\gamma, \gamma_{i} \in\left[-\gamma_{0}, \gamma_{0}\right], i=$ 1,2 , where $B=\left\{W \in E_{r}:|W|_{r} \leq R_{0}\right\}$ for some $R_{0}>0$ :

(B2) The operator $\Phi: B \times\left[-\gamma_{0}, \gamma_{0}\right] \rightarrow F_{q}$ is twice continuously differentiable,

$$
|\Phi(W, \gamma)|_{q+l} \leq c(l)\left(1+\|W\|_{r+l}\right)
$$

and, for $W, U \in E_{r+l}, \gamma \in[-\gamma(l), \gamma(l)]$,

$$
\begin{aligned}
|D(W, U, \gamma)|_{q+l} \leq & c(l)\left(1+\|W\|_{r+l}+\|U\|_{r+l}\right)\|W-U\|_{r}^{2}+ \\
& +c(l)\|W-U\|_{r}\|W-U\|_{r+l},
\end{aligned}
$$


(B3) There exists a family of bounded linear operators $\Lambda(W, \gamma): E_{r} \rightarrow F_{q}$, depending on $(W, \gamma) \in B \times\left[-\gamma_{0}, \gamma_{0}\right]$, with

$$
|\Lambda(W, \gamma) U|_{q} \leq c(0)\|U\|_{r}, \quad U \in E_{r},
$$

that approximates the Fréchet derivative $\Phi_{W}^{\prime}$ as follows. For $W \in E_{r+l} \cap$ $B, \gamma \in[-\gamma(l), \gamma(l)]$ and $U \in E_{r+l}$,

$$
\begin{aligned}
& \left|\Lambda(W, \gamma) U-\Phi_{W}^{\prime}(W, \gamma) U\right|_{q+l} \leq c(l)\left(1+\|W\|_{r+l}\right)|\Phi(W, \gamma)|_{r}\|U\|_{r}+ \\
& +c(l)|\Phi(W, \gamma)|_{r+l}\|U\|_{r}+c(l)|\Phi(W, \gamma)|_{r}\|U\|_{r+l} .
\end{aligned}
$$

(B4) When $W_{i} \in B \cap E_{r+l}, \gamma_{i} \in[-\gamma(l), \gamma(l)], i=1,2$,

$$
\begin{gathered}
\left|\Phi\left(W_{1}, \gamma_{1}\right)-\Phi\left(W_{2}, \gamma_{2}\right)\right|_{q+l} \leq c(l)\left(1+\left\|W_{1}\right\|_{r+l}+\left\|W_{2}\right\|_{r+l}\right) \\
\cdot\left(\left|\gamma_{1}-\gamma_{2}\right|+\left\|W_{1}-W_{2}\right\|_{r}\right)+c(l)\left\|W_{1}-W_{2}\right\|_{r+l}, \\
\left|\left(\Phi_{W}^{\prime}\left(W_{1}, \gamma_{1}\right)-\Phi_{W}^{\prime}\left(W_{2}, \gamma_{2}\right)\right) U\right|_{q+l}+\left|\left(\Lambda\left(W_{1}, \gamma_{1}\right)-\Lambda\left(W_{2}, \gamma_{2}\right)\right) U\right|_{q+l} \leq \\
\leq c(l)\left(\left\|W_{1}-W_{2}\right\|_{r+l}+\left(\left|\gamma_{1}-\gamma_{2}\right|+\left\|W_{1}-W_{2}\right\|_{r}\right) \cdot\right. \\
\left.\quad\left(\left\|W_{1}\right\|_{r+l}+\left\|W_{2}\right\|_{r+l}\right)\right)\|U\|_{r}+c(l)\left(\left|\gamma_{1}-\gamma_{2}\right|+\left\|W_{1}-W_{2}\right\|_{r}\right)\|U\|_{r+l},
\end{gathered}
$$

(B5) Recall that a set $\mathcal{E} \subset \mathbb{R}$ is dense at 0 if meas $(\mathcal{E} \cap[-r, r] / 2 r \rightarrow 1$ as $r \rightarrow 0$. If a set $\mathcal{E} \subset[-\gamma(l), \gamma(l)]$ is dense at 0 and a mapping $\vartheta: \mathcal{E} \rightarrow B \cap E_{r+l}$ is Lipschitz in the sense that for $\gamma_{1}, \gamma_{2} \in \mathcal{E}$,

$$
\left\|\vartheta\left(\gamma_{1}\right)-\vartheta\left(\gamma_{2}\right)\right\|_{r} \leq C\left|\gamma_{1}-\gamma_{2}\right| \text { with } C=C(\vartheta) \text { constant, }
$$

then there is a set $\mathcal{E}(\vartheta) \subset \mathcal{E}$, which is also dense at 0 , such that, for any $\gamma \in \mathcal{E}(\vartheta)$ and $f \in F_{q+l}$, the equation $\Lambda(\vartheta(\gamma), \gamma) \delta W=f$ has a unique solution satisfying

$$
\|\delta W\|_{q-\sigma+l} \leq \frac{1}{|\gamma|} c(l)\left(|f|_{q+l}+\|\vartheta(\gamma)\|_{r+l}|f|_{q}\right) .
$$

(B6) Suppose that $\vartheta_{0}: \mathcal{E}_{0} \rightarrow B \cap E_{r+l}$ and mappings $\vartheta_{k}: \cap_{i=0}^{k-1} \mathcal{E}\left(\vartheta_{i}\right) \rightarrow$ $B \cap E_{r+l}$ satisfy, for a constant $C$ independent of $k \in \mathbb{N}$ sufficiently large,

$$
\begin{gathered}
\left\|\vartheta_{k}\left(\gamma_{1}\right)-\vartheta_{k}\left(\gamma_{2}\right)\right\|_{r} \leq C\left|\gamma_{1}-\gamma_{2}\right|, \quad \gamma_{1}, \gamma_{2} \in \cap_{j=0}^{k-1} \mathcal{E}\left(\vartheta_{j}\right), \\
\left\|\vartheta_{k+1}(\gamma)-\vartheta_{k}(\gamma)\right\|_{r} \leq \frac{1}{2^{k}}, \quad \gamma \in \cap_{j=0}^{k} \mathcal{E}\left(\vartheta_{j}\right) .
\end{gathered}
$$

Then $\cap_{j=0}^{\infty} \mathcal{E}\left(\vartheta_{j}\right)$ is dense at 0 , where the sets $\mathcal{E}\left(\vartheta_{j}\right)$ are defined in (B5).

Theorem 14 Suppose (A1)-(B6) hold and, for $N \in \mathbb{N}$ with $N \geq 2$, equation (12.1) has approximate solution $W=W_{N}(\gamma) \in \cap_{s \in \mathbb{N}_{0}} E_{s}$, with, for a constant $k(N, s)$,

$$
\left\|W_{N}(\gamma)\right\|_{s} \leq k(N, s)|\gamma|, \quad\left|\Phi\left(W_{N}(\gamma), \gamma\right)\right|_{s} \leq k(N, s)|\gamma|^{N+1}
$$

and

$$
\left\|W_{N}\left(\gamma_{1}\right)-W_{N}\left(\gamma_{2}\right)\right\|_{s} \leq k(N, s)\left|\gamma_{1}-\gamma_{2}\right|
$$


Then there is a set $\mathcal{E}$, which is dense at 0 , and a family

$$
\{W=\vartheta(\gamma): \gamma \in \mathcal{E}\} \subset E_{r}
$$

of solutions to (12.1) with $\left\|\vartheta\left(\gamma_{1}\right)-\vartheta\left(\gamma_{2}\right)\right\|_{r} \leq c\left|\gamma_{1}-\gamma_{2}\right|$ for some constant $c$.

In order to apply Theorem 14 to the resolution of the extended system $\widetilde{\mathcal{F}}(U, X, \rho, \nu, \mu, \mathbf{u})=0$, let us choose $\tau \in \widetilde{\mathcal{T}}$ and define

$$
E_{s}=\mathbb{H}_{(S)}^{s} \times\left(H_{o}^{s}\right)^{2} \times \mathbb{R} \times \mathbb{R}^{2}, \quad F_{s}=\mathbb{H}_{(S)}^{s-1} \times\left(H_{e}^{s-1}\right)^{2},
$$

and consider the perturbation $W=(\breve{U}, \breve{\mathcal{W}}, \breve{\mu}, \breve{\mathbf{u}})$ defined as in $(6.3)$, and $\Phi$ defined by

$$
\begin{aligned}
\Phi(W, \gamma)=\frac{1}{|\varepsilon|^{2 m}} \widetilde{\mathcal{F}}\left(U_{4 m}+|\varepsilon|^{2 m} \breve{U}, X_{4 m}+|\varepsilon|^{2 m} \breve{\mathcal{W}},\right. \\
\\
\left.\lambda+\gamma, \nu, \mu_{4 m}+|\varepsilon|^{2 m} \breve{\mu}, \mathbf{u}_{4 m}+|\varepsilon|^{2 m} \breve{\mathbf{u}}\right)
\end{aligned}
$$

in which we replace the parameter $\varepsilon=\left(\varepsilon_{1}, \varepsilon_{2}\right)$ by $(\rho, \nu)$ by using the diffeomorphism $\left(\varepsilon_{1}^{2}, \varepsilon_{2}^{2}\right) \mapsto\left(\rho_{4 m}(\varepsilon), \nu_{4 m}(\varepsilon)\right)$ from a neighborhood of 0 in $\mathbb{R}^{+2}$ into a neighborhood of $\left(\lambda, \nu_{c}\right)$ in $\mathbb{R}^{2}$. This is possible thanks to condition $(6.4)$ which is satisfied for $\tau \in \widetilde{\mathcal{T}}$. The loss of regularity at the origin in $\gamma$, due to the square roots, is not a problem here, because the scaling we made gives a perturbation of order $|\gamma|^{m}$, and we only need the perturbation to be Lipschitz in $\gamma$. Then for $\nu$ fixed close enough to $\nu_{c}$, and $\gamma$ close to 0 , the function $\Phi(\cdot, \gamma)$ maps smoothly $E_{s}$ to $F_{s-1}$ for $s \geq 4$, and $\Phi$ is Lipschitz in $\gamma$ near 0 . A smoothing operator with the required properties can be defined as

$$
S_{\wp} u=\frac{1}{|\Omega|^{1 / 2}} \sum_{\mathbf{k} \in \Gamma^{\prime}} v(\wp|\mathbf{k}|) \widehat{u}_{\mathbf{k}} e^{i \mathbf{k} \cdot X},
$$

where $v: \mathbb{R}^{+} \mapsto \mathbb{R}^{+}$is a smooth function which equals 1 on $[0,1]$ and 0 on $[2,+\infty)$.

By construction $W_{N}(\gamma)=0$ with $N=2 m$. Hence (12.9) and (12.10) are satisfied. Applying the same arguments as in section 9 of [15], we conclude from the smoothness of $\Phi$, that for $10 \leq q \leq r-24$, the operator $\Phi$ satisfies Conditions (B1) and (B2), and inequality (12.7) from Condition (B4). Let us denote by $\Lambda(W, \gamma)$ the approximate differential for which the inversion corresponds to invert the system (7.17), (7.18), (7.19). The estimates (12.5) and (12.7) are verified for $q \leq r-1$ and the rest of the differential, given by

$$
\overline{\mathcal{R}}(W, \gamma)[\delta W]=\left(\overline{\mathcal{R}}_{1}[u, \varsigma], \overline{\mathcal{R}}_{2}[u], X^{\prime} \overline{\mathcal{R}}_{3}[u, \varsigma, v, 0, \delta \mu, \delta \mathbf{u}]\right)
$$

satisfies (12.6) as may be easily proved in following the same lines as for proving Lemma 4 (roughly speaking we loose 2 derivatives in $W$, one derivative in $\Phi$, and two derivatives in $\delta W$ which is OK with $q \leq r-1$ ). So Conditions (B3) and (B4) are satisfied. Now, taking into account the result of Theorem 13, it appears that Condition (B5) with (12.8) hold with $\sigma=10$ and $r \geq q+24, q \geq 10$. Condition (B6) is trivially satisfied here since the set 
$\mathcal{E}^{\prime}(\nu)$, defined in Theorem 11, is independent of the iteration point, which means that all $\mathcal{E}\left(\vartheta_{j}\right)$ are identical. Hence Theorem 14 applies, leading to the existence of a solution of $\Phi(W, \gamma)=0$ in $E_{r}$. Coming back to the variables $U, \mu, \mathbf{u}$, and coming back to the parameters $\left(\varepsilon_{1}, \varepsilon_{2}\right)$ instead of $(\rho, \nu)$, gives Theorem 2 after the change of notation $\tilde{\mathcal{T}}$ into $\mathcal{T}$.

\subsection{Directional Stokes drift - Proof of Lemma 3}

The diffeomorphism (5.1) transforms the family of lines parallel to the direction $\varrho$ into integral curves of the vector field $V$. Hence these integral curves are given in parametric form by (for any fixed $\delta \in \mathbb{R}$ )

$$
X=\mathbb{T}^{-1} Y_{\delta}(s)+\mathcal{W}\left(Y_{\delta}(s), \varepsilon\right), \quad Y_{\delta}(s)=(s+\delta, \rho s), s \in \mathbb{R},
$$

hence

$$
x_{1}=\frac{\lambda \tau_{2}(s+\delta)+\rho \tau_{1} s}{\lambda\left(\tau_{1}+\tau_{2}\right)}+w_{1}\left(Y_{\delta}(s), \varepsilon\right), x_{2}=\frac{\lambda(s+\delta)-\rho s}{\lambda\left(\tau_{1}+\tau_{2}\right)}+w_{2}\left(Y_{\delta}(s), \varepsilon\right),
$$

where $w_{1}$ and $w_{2}$ are odd periodic functions of $Y$. It is then clear that these curves oscillate around lines of slope

$$
\begin{aligned}
& \frac{\lambda-\rho}{\lambda \tau_{2}+\rho \tau_{1}}=-\frac{\rho_{1} \varepsilon_{1}^{2}+\rho_{2} \varepsilon_{2}^{2}}{\lambda\left(\tau_{1}+\tau_{2}\right)}+O\left(|\varepsilon|^{4}\right) \\
& \quad=\beta_{1} \varepsilon_{1}^{2}+\beta_{2} \varepsilon_{2}^{2}-4 \tau_{1}\left(\mu_{c}\right)^{-2} \varepsilon_{1}^{2}+4 \lambda^{4} \tau_{2}\left(\mu_{c}\right)^{-2} \varepsilon_{2}^{2}+O\left(|\varepsilon|^{4}\right)
\end{aligned}
$$

trajecDirection

by using (B.9) and (B.10). Now consider the direction of propagation $\mathbf{u}$ of the travelling waves, which corresponds to the direction of the velocity of particles as $x_{3} \rightarrow-\infty$. We have, thanks to Theorem $4, \mathbf{u}=\left(1+\omega_{1}, \omega_{2}\right)$ with $\omega_{1}=O\left(|\varepsilon|^{4}\right)$. The slope of $\mathbf{u}$ is then

$$
\omega_{2}\left(1+\omega_{1}\right)^{-1}=\beta_{1} \varepsilon_{1}^{2}+\beta_{2} \varepsilon_{2}^{2}+O\left(|\varepsilon|^{4}\right) .
$$

Comparing (12.11) and (12.12), this proves Lemma 3, since we proved the existence of the travelling waves and diffeomorphism $X$ which correspond to the previous asymptotic expansions. Expressing the difference between (12.11) and (12.12) in terms of $\left(\rho-\lambda, \nu-\nu_{c}\right)$, then for a fixed eligible $\nu$, we can use a fixed point argument to find $\rho$ as a function of $\nu$ which cancels this difference (expression analytic in $(\rho-\lambda)$ plus a small Lipschitz term). Then coming back to the $\left(\varepsilon_{1}, \varepsilon_{2}\right)$ plane this proves the claim of Lemma 3 on the special ratio $\varepsilon_{1} / \varepsilon_{2}$ in fact function of $\varepsilon_{2}^{2}$ at higher orders. Notice that the angle is 0 in the case $\tau_{1}=\tau_{2}(\lambda=1)$ of a diamond pattern, and $\varepsilon_{1}=\varepsilon_{2}$ (symmetric waves as in [16]). 


\section{A Formal computation of asymmetrical waves}

Let us write formally the nonlinear system (1.2), (1.3), (1.4) under the form

$$
\mathcal{L}_{0} U+\tilde{\mu} \mathcal{L}_{1} U+\mathcal{L}_{2}(\boldsymbol{\omega}, U)+\mathcal{N}_{2}(U, U)+\mathcal{N}_{3}(U, U, U)+O\left(\|U\|^{4}+|\boldsymbol{\omega}|\|U\|^{2}\right)=0,
$$

with $U=(\psi, \eta), \tilde{\mu}=\mu-\mu_{c}, \boldsymbol{\omega}=\mathbf{u}-\mathbf{u}_{0}$

$$
\begin{gathered}
\mathcal{L}_{1} U=(0, \eta), \quad \mathcal{L}_{2}(\boldsymbol{\omega}, U)=(-\boldsymbol{\omega} \cdot \nabla \eta, \boldsymbol{\omega} \cdot \nabla \psi), \\
\mathcal{N}_{2}(U, U)=\left(\begin{array}{c}
\mathcal{G}^{(1)}\{\eta\} \psi \\
\frac{1}{2} \nabla \psi^{2}-\frac{1}{2}\left(\frac{\partial \eta}{\partial x_{1}}\right)^{2}
\end{array}\right), \quad \mathcal{N}_{3}(U, U, U)=\left(\begin{array}{c}
\mathcal{G}^{(2)}\{\eta, \eta\} \psi \\
-\frac{\partial \eta}{\partial x_{1}}(\nabla \eta \cdot \nabla \psi)
\end{array}\right),
\end{gathered}
$$

where (see [16])

$$
\begin{aligned}
\mathcal{G}^{(1)}\{\eta\} \psi & =-\mathcal{G}^{(0)}\left(\eta \mathcal{G}^{(0)} \psi\right)-\nabla \cdot(\eta \nabla \psi), \\
\mathcal{G}^{(2)}\{\eta, \eta\} \psi & =\mathcal{G}^{(0)}\left(\eta \mathcal{G}^{(0)}\left(\eta \mathcal{G}^{(0)} \psi\right)\right)+\frac{1}{2} \mathcal{G}^{(0)}\left(\eta^{2} \Delta \psi\right)+\frac{1}{2} \Delta\left(\eta^{2} \mathcal{G}^{(0)} \psi\right)
\end{aligned}
$$

Notice that operators $\mathcal{L}_{0}, \mathcal{L}_{1}, \mathcal{L}_{2}(\boldsymbol{\omega}, \cdot), \mathcal{N}_{2}, \mathcal{N}_{3}$ commute with the symmetries $\mathcal{T}_{\mathbf{v}}$ and $\mathcal{S}_{0}$

Formal Fredholm alternative Let us consider the formal resolution of the linear system $\mathcal{L}_{0} U=F=(f, g)$, with

$$
\begin{aligned}
& U=\frac{1}{|\Omega|^{1 / 2}} \sum_{\mathbf{k} \in \Gamma^{\prime}} \widehat{U}_{\mathbf{k}} e^{i \mathbf{k} \cdot X}, \quad \widehat{U}_{\mathbf{k}}=\left(\widehat{\psi}_{\mathbf{k}}, \widehat{\eta}_{\mathbf{k}}\right), \quad \widehat{\psi}_{\mathbf{0}}=0 \\
& F=\frac{1}{|\Omega|^{1 / 2}} \sum_{\mathbf{k} \in \Gamma^{\prime}} \widehat{F}_{\mathbf{k}} e^{i \mathbf{k} \cdot X}, \quad \widehat{F}_{\mathbf{k}}=\left(\widehat{f}_{\mathbf{k}}, \widehat{g}_{\mathbf{k}}\right), \quad \widehat{f}_{\mathbf{0}}=0 .
\end{aligned}
$$

Then, we have for $\left\{\mathbf{k} \cdot \mathbf{u}_{0}\right\}^{2} \neq \mu_{c}|\mathbf{k}|$ i.e. by assumption for $\mathbf{k} \neq \mathbf{0}, \pm K_{1}, \pm K_{2}$, this leads to

$$
\psi_{\mathbf{k}}=-\frac{\mu_{c} \widehat{f}_{\mathbf{k}}+i\left(\mathbf{k} \cdot \mathbf{u}_{0}\right) \widehat{g}_{\mathbf{k}}}{\left(\mathbf{k} \cdot \mathbf{u}_{0}\right)^{2}-\mu_{c}|\mathbf{k}|}, \quad \eta_{\mathbf{k}}=\frac{i\left(\mathbf{k} \cdot \mathbf{u}_{0}\right) \widehat{f}_{\mathbf{k}}-|\mathbf{k}| \widehat{g}_{\mathbf{k}}}{\left(\mathbf{k} \cdot \mathbf{u}_{0}\right)^{2}-\mu_{c}|\mathbf{k}|}
$$

and $\psi_{\mathbf{0}}=0, \eta_{\mathbf{0}}=\mu_{c}{ }^{-1} g_{\mathbf{0}}$ for $\mathbf{k}=\mathbf{0}$, while for $\mathbf{k}= \pm K_{1}, \pm K_{2}$, we need to satisfy the compatibility conditions

$$
\left(F, \zeta_{K_{1}}\right)=\left(F, \bar{\zeta}_{K_{1}}\right)=\left(F, \zeta_{K_{2}}\right)=\left(F, \bar{\zeta}_{K_{2}}\right)=0
$$

which gives

$$
\mu_{c} f_{ \pm K_{1}} \pm i g_{ \pm K_{1}}=0, \quad \mu_{c} f_{ \pm K_{2}} \pm i \lambda g_{ \pm K_{2}}=0 .
$$

To insure the uniqueness of the definition of the pseudo-inverse $\widetilde{\mathcal{L}}_{0}^{-1}$, we fix $U$ such that

$$
\left(U, \zeta_{K_{1}}\right)=\left(U, \bar{\zeta}_{K_{1}}\right)=\left(U, \zeta_{K_{2}}\right)=\left(U, \bar{\zeta}_{K_{2}}\right)=0,
$$

hence this leads to

$$
\psi_{K_{j}}=\frac{1}{\mu_{c}\left(2+\tau_{j}^{2}\right)} f_{K_{j}}, \quad \eta_{K_{1}}=\frac{i}{2+\tau_{1}^{2}} f_{K_{1}}, \quad \eta_{K_{2}}=\frac{i}{\lambda\left(2+\tau_{2}^{2}\right)} f_{K_{2}} .
$$


Bifurcation equation Now coming back to (A.1), we use a formal Lyapunov Schmidt method and decompose $U$ as $U=H+M$, where $M \in \operatorname{ker} \mathcal{L}_{0}^{\perp}$ and

$$
H=A \zeta_{K_{1}}+\overline{A \zeta}_{K_{1}}+B \zeta_{K_{2}}+\overline{B \zeta}_{K_{2}}=\mathcal{P}_{0} U \in \operatorname{ker} \mathcal{L}_{0}
$$

We can solve formally with respect to $M$ the part of equ. (A.1) orthogonal to the 4-dimensional kernel of $\mathcal{L}_{0}$, as a uniquely determined formal power series in $\boldsymbol{\omega}, \tilde{\mu}, A, \bar{A}, B, \bar{B}$, which we write as

$$
M=\mathcal{M}(\tilde{\mu}, \boldsymbol{\omega}, A, \bar{A}, B, \bar{B}) .
$$

The uniqueness of the series, the symmetries of our system and the action of the symmetries on the eigenvectors, lead to the following identities

$$
\begin{aligned}
& \mathcal{T}_{\mathbf{v}} \mathcal{M}(\tilde{\mu}, \boldsymbol{\omega}, A, \bar{A}, B, \bar{B})=\mathcal{M}\left(\tilde{\mu}, \boldsymbol{\omega}, A e^{i K_{1} \cdot \mathbf{v}}, \bar{A} e^{-i K_{1} \cdot \mathbf{v}}, B e^{i K_{2} \cdot \mathbf{v}}, \bar{B} e^{-i K_{2} \cdot \mathbf{v}}\right), \\
& \mathcal{S}_{0} \mathcal{M}(\tilde{\mu}, \boldsymbol{\omega}, A, \bar{A}, B, \bar{B})=\mathcal{M}(\tilde{\mu}, \boldsymbol{\omega}, \bar{A}, A, \bar{B}, B) .
\end{aligned}
$$

The principal part of $\mathcal{M}$ is given by

$$
\begin{aligned}
\mathcal{M}= & -\widetilde{\mathcal{L}}_{0}^{-1} \mathcal{N}_{2}(H, H)+O\left\{(|\tilde{\mu}|+|\boldsymbol{\omega}|)\|H\|+\|H\|^{3}\right\}, \\
-\widetilde{\mathcal{L}}_{0}^{-1} \mathcal{N}_{2}(H, H)= & A^{2} U_{2000}+|A|^{2} U_{1100}+\bar{A}^{2} U_{0200}+A B U_{1010}+\bar{A} B U_{0110}+ \\
& +A \bar{B} U_{1001}+\overline{A B} U_{0101}+B^{2} U_{0020}+|B|^{2} U_{0011}+\bar{B}^{2} U_{0002}
\end{aligned}
$$

where we observe, because of the non resonance assumption ( $\operatorname{ker} \mathcal{L}_{0}$ is only 4dimensional), that $\mathcal{P}_{0} \mathcal{N}_{2}(H, H)=0$. Using (A.2) we find

$$
\begin{aligned}
\mathcal{N}_{2}(H, H)= & A^{2} V_{2000}+|A|^{2} V_{1100}+\bar{A}^{2} V_{0200}+A B V_{1010}+\bar{A} B V_{0110}+ \\
& +A \bar{B} V_{1001}+\overline{A B} V_{0101}+B^{2} V_{0020}+|B|^{2} V_{0011}+\bar{B}^{2} V_{0002}
\end{aligned}
$$

with

$$
\begin{aligned}
V_{2000}= & \left(0, \frac{1}{\mu_{c}^{2}}\right) e^{2 i K_{1} \cdot X}, \quad V_{0020}=\left(0, \frac{\lambda^{4}}{\mu_{c}^{2}}\right) e^{2 i K_{2} \cdot X} \\
V_{1100}= & 0, \quad V_{0011}=0, \quad V_{0200}=\bar{V}_{2000}, \quad V_{0002}=\bar{V}_{0020} \\
V_{1010}= & \left\{\frac{i \lambda(\lambda+1)}{\mu_{c}}\left(1-\tau_{1} \tau_{2}+\frac{\lambda^{2}-\lambda+1}{\mu_{c}^{2}}-\frac{\left|K_{1}+K_{2}\right|}{\mu_{c}}\right),\right. \\
& \lambda\left(1-\tau_{1} \tau_{2}+\frac{\lambda}{\mu_{c}^{2}}\right\} e^{\left.i\left(K_{1}+K_{2}\right) \cdot X\right)}, \\
V_{1001}= & \left\{\frac{i \lambda(1-\lambda)}{\mu_{c}}\left(1-\tau_{1} \tau_{2}+\frac{\lambda^{2}+\lambda+1}{\mu_{c}^{2}}-\frac{\left|K_{1}-K_{2}\right|}{\mu_{c}}\right),\right. \\
& \lambda\left(1-\tau_{1} \tau_{2}-\frac{\lambda}{\mu_{c}^{2}}\right\} e^{\left.i\left(K_{1}-K_{2}\right) \cdot X\right)}, \\
V_{0101}= & \bar{V}_{1010}, \quad V_{1001}=\bar{V}_{0110 .} .
\end{aligned}
$$

Now, thanks to (A.3) we obtain $-\widetilde{\mathcal{L}}_{0}^{-1} \mathcal{N}_{2}(H, H)$ as follows

$$
\begin{aligned}
& U_{2000}=\left(\frac{i}{\mu_{c}^{2}}, \frac{1}{\mu_{c}^{3}}\right) e^{2 i K_{1} \cdot X}, \quad U_{0020}=\left(\frac{i \lambda^{3}}{\mu_{c}^{2}}, \frac{\lambda^{4}}{\mu_{c}^{3}}\right) e^{2 i K_{2} \cdot X}, \\
& U_{1100}=0, \quad U_{0011}=0, \quad U_{0200}=\bar{U}_{2000}, \quad U_{0002}=\bar{U}_{0020}, \\
& U_{1010}=\left(i \alpha_{1010}, \beta_{1010}\right) e^{i\left(K_{1}+K_{2}\right) \cdot X} \\
& U_{1001}=\left(i \alpha_{1001}, \beta_{1001}\right) e^{i\left(K_{1}-K_{2}\right) \cdot X} \\
& U_{0101}=\bar{U}_{1010}, \quad U_{1001}=\bar{U}_{0110},
\end{aligned}
$$


where

$$
\begin{gathered}
\alpha_{1010}=\frac{\lambda(\lambda+1)}{\mu_{c}^{2}}+\frac{2 \lambda(\lambda+1)}{D_{+}}\left(1-\tau_{1} \tau_{2}-\frac{\lambda}{\mu_{c}^{2}}\right) \\
\alpha_{1001}=\frac{\lambda(1-\lambda)}{\mu_{c}^{2}}+\frac{2 \lambda(1-\lambda)}{D_{-}}\left(1-\tau_{1} \tau_{2}+\frac{\lambda}{\mu_{c}^{2}}\right) \\
\beta_{1010}=-\frac{\lambda}{\mu_{c}}\left(1-\frac{2(\lambda+1)^{2}}{D_{+}}\right)\left(1-\tau_{1} \tau_{2}-\frac{\lambda}{\mu_{c}^{2}}\right)+\frac{\lambda\left(\lambda^{2}+1\right)}{\mu_{c}^{3}}, \\
\beta_{1001}=-\frac{\lambda}{\mu_{c}}\left(1-\frac{2(\lambda-1)^{2}}{D_{-}}\right)\left(1-\tau_{1} \tau_{2}+\frac{\lambda}{\mu_{c}^{2}}\right)+\frac{\lambda\left(\lambda^{2}+1\right)}{\mu_{c}^{3}} \\
D_{ \pm}=(1 \pm \lambda)^{2}-\mu_{c}\left|K_{1} \pm K_{2}\right|
\end{gathered}
$$

Replacing $M$ by $\mathcal{M}(\tilde{\mu}, \boldsymbol{\omega}, A, \bar{A}, B, \bar{B})$ in the compatibility conditions, i.e. the components of (A.1) on ker $\mathcal{L}_{0}$,

$$
\left\langle\tilde{\mu} \mathcal{L}_{1}(H+\mathcal{M})+\mathcal{L}_{2}(\boldsymbol{\omega}, H+\mathcal{M})+\mathcal{N}_{2}(H+\mathcal{M}, H+\mathcal{M})+\ldots, \zeta_{K_{j}}\right\rangle=0,
$$

for $j=1,2$, and noticing that the complex conjugate equations are automatically satisfied, lead to two complex equations of the form

$$
f(\tilde{\mu}, \boldsymbol{\omega}, A, \bar{A}, B, \bar{B})=0, \quad g(\tilde{\mu}, \boldsymbol{\omega}, A, \bar{A}, B, \bar{B})=0,
$$

for which the equivariance of the system (A.1) with respect to symmetries $\mathcal{T}_{\mathbf{v}}$ and $\mathcal{S}_{0}$, lead to the following properties

$$
\begin{aligned}
f\left(\tilde{\mu}, \boldsymbol{\omega}, A e^{i K_{1} \cdot \mathbf{v}}, \bar{A} e^{-i K_{1} \cdot \mathbf{v}}, B e^{i K_{2} \cdot \mathbf{v}}, \bar{B} e^{-i K_{2} \cdot \mathbf{v}}\right) & =e^{i K_{1} \cdot \mathbf{v}} f(\tilde{\mu}, \boldsymbol{\omega}, A, \bar{A}, B, \bar{B}) \\
g\left(\tilde{\mu}, \boldsymbol{\omega}, A e^{i K_{1} \cdot \mathbf{v}}, \bar{A} e^{-i K_{1} \cdot \mathbf{v}}, B e^{i K_{2} \cdot \mathbf{v}}, \bar{B} e^{-i K_{2} \cdot \mathbf{v}}\right) & =e^{i K_{2} \cdot \mathbf{v}} g(\tilde{\mu}, \boldsymbol{\omega}, A, \bar{A}, B, \bar{B}) \\
f(\tilde{\mu}, \boldsymbol{\omega}, \bar{A}, A, \bar{B}, B) & =\bar{f}(\tilde{\mu}, \boldsymbol{\omega}, A, \bar{A}, B, \bar{B}) \\
g(\tilde{\mu}, \boldsymbol{\omega}, \bar{A}, A, \bar{B}, B) & =\bar{g}(\tilde{\mu}, \boldsymbol{\omega}, A, \bar{A}, B, \bar{B}) .
\end{aligned}
$$

Since the wave vectors $K_{1}$ and $K_{2}$ are linearly independent, it results that $f$ and $g$ take formally the form

$$
\begin{aligned}
& f(\tilde{\mu}, \boldsymbol{\omega}, A, \bar{A}, B, \bar{B})=A \phi_{1}\left(\tilde{\mu}, \boldsymbol{\omega},|A|^{2},|B|^{2}\right) \\
& g(\tilde{\mu}, \boldsymbol{\omega}, A, \bar{A}, B, \bar{B})=B \phi_{2}\left(\tilde{\mu}, \boldsymbol{\omega},|A|^{2},|B|^{2}\right),
\end{aligned}
$$

where functions $\phi_{1}$ and $\phi_{2}$ are real valued. It results immediately that we have the following (formal) solutions of our system (in addition to the trivial solution 0 ):

i) $B=0,|A|$ satisfying $\phi_{1}\left(\tilde{\mu}, \boldsymbol{\omega},|A|^{2}, 0\right)=0$, which is the 2-dimensional travelling wave with basic wave vector $K_{1}$, and where, with no loss of generality, we can choose the velocity $\mathbf{c}$ in the direction of $K_{1}$.

ii) $A=0, \quad|B|$ satisfying $\phi_{2}\left(\tilde{\mu}, \boldsymbol{\omega}, 0,|B|^{2}\right)=0$, which is the 2-dimensional travelling wave with basic wave vector $K_{2}$, and where, with no loss of generality, we can choose the velocity $\mathbf{c}$ in the direction of $K_{2}$.

iii) $|A|$ and $|B|$ such that

$$
\phi_{1}\left(\tilde{\mu}, \boldsymbol{\omega},|A|^{2},|B|^{2}\right)=0 \quad, \phi_{2}\left(\tilde{\mu}, \boldsymbol{\omega},|A|^{2},|B|^{2}\right)=0,
$$

which gives a family of asymmetrical 3-dimensional travelling waves. This system has the following form for leading terms

$$
\begin{gathered}
0=\mu_{c}^{-1} \widetilde{\mu}-2\left(K_{1} \cdot \boldsymbol{\omega}\right)+a_{1}|A|^{2}+b_{1}|B|^{2}+O\left\{\left(|\tilde{\mu}|+|\boldsymbol{\omega}|+|A|^{2}+|B|^{2}\right)^{2}\right\}, \\
0=\mu_{c}^{-1} \widetilde{\mu}-2 \lambda^{-1}\left(K_{2} \cdot \boldsymbol{\omega}\right)+a_{2}|A|^{2}+b_{2}|B|^{2}+O\left\{\left(|\tilde{\mu}|+|\boldsymbol{\omega}|+|A|^{2}+|B|^{2}\right)^{2}\right\} .
\end{gathered}
$$


Indeed, the leading terms of $\phi_{1}$ and $\phi_{2}$ independent of $|A|$ and $|B|$ come from

$$
\begin{aligned}
& \left\langle\tilde{\mu} \mathcal{L}_{1} \zeta_{K_{1}}+\mathcal{L}_{2}\left(\boldsymbol{\omega}, \zeta_{K_{1}}\right), \zeta_{K_{1}}\right\rangle=|\Omega|\left(\mu_{c}\right)^{-1}\left(\tilde{\mu}\left(\mu_{c}\right)^{-1}-2 \omega \cdot K_{1}\right), \\
& \left\langle\tilde{\mu} \mathcal{L}_{1} \zeta_{K_{2}}+\mathcal{L}_{2}\left(\boldsymbol{\omega}, \zeta_{K_{2}}\right), \zeta_{K_{2}}\right\rangle=|\Omega|\left(\mu_{c}\right)^{-1}\left(\lambda^{2} \tilde{\mu}\left(\mu_{c}\right)^{-1}-2 \lambda \omega \cdot K_{2}\right) .
\end{aligned}
$$

The coefficients $a_{j}, b_{j}$ given by

$$
\begin{aligned}
& a_{1}=\mu_{c}|\Omega|^{-1}\left\langle 2 \mathcal{N}_{2}\left(\bar{\zeta}_{K_{1}}, U_{2000}\right)+3 \mathcal{N}_{3}\left(\zeta_{K_{1}}, \zeta_{K_{1}}, \bar{\zeta}_{K_{1}}\right), \zeta_{K_{1}}\right\rangle, \\
& b_{1}=\mu_{c}|\Omega|^{-1}\left\langle 2 \mathcal{N}_{2}\left(\zeta_{K_{2}}, U_{1001}\right)+2 \mathcal{N}_{2}\left(\bar{\zeta}_{K_{2}}, U_{1010}\right)+6 \mathcal{N}_{3}\left(\zeta_{K_{1}}, \zeta_{K_{2}}, \bar{\zeta}_{K_{2}}\right), \zeta_{K_{1}}\right\rangle, \\
& a_{2}=\mu_{c}\left(\lambda^{2}|\Omega|\right)^{-1}\left\langle 2 \mathcal{N}_{2}\left(\zeta_{K_{1}}, U_{0110}\right)+2 \mathcal{N}_{2}\left(\bar{\zeta}_{K_{1}}, U_{1010}\right)+6 \mathcal{N}_{3}\left(\zeta_{K_{1}}, \zeta_{K_{2}}, \bar{\zeta}_{K_{1}}\right), \zeta_{K_{2}}\right\rangle, \\
& b_{2}=\mu_{c}\left(\lambda^{2}|\Omega|\right)^{-1}\left\langle 2 \mathcal{N}_{2}\left(\bar{\zeta}_{K_{2}}, U_{0020}\right)+3 \mathcal{N}_{3}\left(\zeta_{K_{2}}, \zeta_{K_{2}}, \bar{\zeta}_{K_{2}}\right), \zeta_{K_{2}}\right\rangle,
\end{aligned}
$$

are computed in the next paragraph. We can formally solve the system of equations (A.9) with respect to $\tilde{\mu}$ and

$$
\omega_{2}=\left(\tau_{1}+\tau_{2}\right)^{-1} \boldsymbol{\omega} \cdot\left(K_{1}-\lambda^{-1} K_{2}\right) .
$$

Indeed, we obtain respectively in adding and subtracting the two equations, a system easy to solve, in taking into account the fact that $\mathbf{u}=\left(1+\omega_{1}, \omega_{2}\right)$ has a unit length, and by using $\varepsilon_{1}=|A|, \quad \varepsilon_{2}=|B|$. We then obtain at main order

$$
\begin{aligned}
\frac{\tilde{\mu}}{\mu_{c}} & =-\frac{\left(\tau_{1} a_{2}+\tau_{2} a_{1}\right)}{\tau_{1}+\tau_{2}} \varepsilon_{1}^{2}-\frac{\left(\tau_{1} b_{2}+\tau_{2} b_{1}\right)}{\tau_{1}+\tau_{2}} \varepsilon_{2}^{2}+O\left\{\left(\varepsilon_{1}^{2}+\varepsilon_{2}^{2}\right)^{2}\right\}, \\
\boldsymbol{\omega} & =\left(\omega_{1}, \omega_{2}\right), \omega_{1}=-\frac{\omega_{2}^{2}}{2}+O\left(\omega_{2}^{4}\right), \\
\omega_{2} & =\frac{\left(a_{1}-a_{2}\right)}{2\left(\tau_{1}+\tau_{2}\right)} \varepsilon_{1}^{2}+\frac{\left(b_{1}-b_{2}\right)}{2\left(\tau_{1}+\tau_{2}\right)} \varepsilon_{2}^{2}+O\left\{\left(\varepsilon_{1}^{2}+\varepsilon_{2}^{2}\right)^{2}\right\} .
\end{aligned}
$$

The phases of $A$ and $B$ may be changed independently in using the shift $\mathcal{T}_{\mathbf{v}}$. Thanks to (A.4), in choosing $A$ and $B$ real we have $\mathcal{S}_{0} \mathcal{M}=\mathcal{M}$, hence $\mathcal{S}_{0} U=U$. Then, for any fixed pair $\left(\varepsilon_{1}, \varepsilon_{2}\right)$ we have a torus of solutions, which is generated by acting the operator $\mathcal{T}_{\mathbf{v}}$ on the particular solution obtained with $A$ and $B$ real. This twoparameter family of tori of 3 -dimensional waves connects with the 2-dimensional travelling waves respectively of wave vectors $K_{1}$ and $K_{2}$. This ends the proof of theorem 4 with

$$
\begin{gathered}
\alpha_{1}=-\mu_{c} \frac{\tau_{2} a_{1}+\tau_{1} a_{2}}{\tau_{1}+\tau_{2}}, \quad \alpha_{2}=-\mu_{c} \frac{\tau_{2} b_{1}+\tau_{1} b_{2}}{\tau_{1}+\tau_{2}}, \\
\beta_{1}=\frac{a_{1}-a_{2}}{2\left(\tau_{1}+\tau_{2}\right)}, \quad \beta_{2}=\frac{b_{1}-b_{2}}{2\left(\tau_{1}+\tau_{2}\right)} .
\end{gathered}
$$

Computation of coefficients $a_{j}, b_{j}$. Using (A.2) and the expressions of $U_{\text {pqrs }}$ found in (A.6,A.7,A.8), we easily compute the scalar products occuring in (A.10). It then results that

$$
\begin{gathered}
a_{1}=\frac{4}{\mu_{c}^{4}}, \quad b_{2}=\frac{4 \lambda^{6}}{\mu_{c}^{4}}, \quad b_{1}=\lambda^{2} a_{2}, \\
a_{2}=\frac{4(1+\lambda)^{2}}{D_{+}}\left(1-\tau_{1} \tau_{2}-\frac{\lambda}{\mu_{c}^{2}}\right)^{2}+\frac{4(1-\lambda)^{2}}{D_{-}}\left(1-\tau_{1} \tau_{2}+\frac{\lambda}{\mu_{c}^{2}}\right)^{2}+ \\
+\frac{8\left(\lambda^{2}+1\right)}{\mu_{c}^{2}}\left(1-\tau_{1} \tau_{2}\right)-\frac{6 \lambda^{2}}{\mu_{c}^{4}}-2\left(1-\tau_{1} \tau_{2}\right)^{2} .
\end{gathered}
$$




\section{B Computation of $\rho$ and $\nu$ in Lemma 5}

Computation of $\rho$. We start with equation (5.10) where the unknown are the diffeomorphism $X(Y)$, and scalars $\rho$ and $\nu$. With the notation

$$
Y(X)=\mathbb{T} X+\mathcal{V}(X, \varepsilon), \quad \mathcal{V}=\left(v_{1}, v_{2}\right), \quad v_{3}=v_{2}-\rho v_{1},
$$

for the inverse diffeomorphism, this equation may be written as

$$
V \cdot \nabla_{X} Y=-\left|Y^{\prime}(X)\right|^{1 / 3}(\nu \mathfrak{a})^{1 / 3}(\mathbb{Q} V \cdot V)^{1 / 6} \varrho
$$

where $\nabla_{X} Y$ is transpose of the Jacobi matrix $Y^{\prime}$. Note that the rotation number $\varrho$ is a topological invariant. It depends only on the vector field $V$ and does not depend on the multiplier in front of $\varrho$ in the right hand side of (B.2). Hence $\rho$ can be determined from the relation $V \cdot \nabla Y \cdot \varrho^{\perp}=0$. Recalling (B.1) and noting that the lines of the matrix $\mathbb{T}$ coinside with the wave vectors $K_{i}$ we can write this relation in the equivalent form

$$
V \cdot\left(K_{2}-\rho K_{1}\right)+V \cdot \nabla_{X} v_{3}=0 .
$$

Let us define new coordinates $X^{\prime}=\left(K_{1} \cdot X, K_{2} \cdot X\right)$ and set

$$
W=\left(K_{1} \cdot V, K_{2} \cdot V\right)\left(X\left(X^{\prime}\right)\right), \quad v_{3}^{\prime}\left(K_{1} \cdot X, K_{2} \cdot X\right)=v_{3}(X), \quad W_{0}=W_{2} / W_{1} .
$$

The functions $v_{3}^{\prime}$ and $W_{0}$ are $2 \pi$ periodic in $x_{i}^{\prime}$. Moreover $W_{0}$ is even and is invariant under the symmetries $\tilde{\mathcal{S}}_{j}, j=1,2$. In this notation equation (B.3) reads

$$
\partial_{x_{1}^{\prime}} v_{3}^{\prime}+W_{0} \partial_{x_{2}^{\prime}} v_{3}^{\prime}=\rho-W_{0} .
$$

and let us now compute the principal part of $W_{0}$. From Theorem 4 we have for the components $(\psi, \eta)$ of $U$

$$
\begin{aligned}
\nabla \psi= & -2 K_{1} \varepsilon_{1} \cos x_{1}^{\prime}-2 K_{2} \varepsilon_{2} \cos x_{2}^{\prime}-\frac{4}{\mu_{c}^{2}} \varepsilon_{1}^{2} \cos 2 x_{1}^{\prime}+ \\
& -\frac{4 \lambda^{3}}{\mu_{c}^{2}} \varepsilon_{2}^{2} \cos 2 x_{2}^{\prime}+\varepsilon_{1} \varepsilon_{2} \nabla \psi^{(1,1)}+O_{e}\left(|\varepsilon|^{3}\right)
\end{aligned}
$$

where $O_{e}\left(|\varepsilon|^{p}\right)$ (resp. $\left.O_{o}\left(|\varepsilon|^{p}\right)\right)$ means terms even (resp. odd) in $\left(x_{1}^{\prime}, x_{2}^{\prime}\right)$ of order $|\varepsilon|^{p}$, and

$$
\begin{aligned}
\nabla \psi^{(1,1)}= & -2\left(K_{1}+K_{2}\right) \alpha_{1010} \cos \left(x_{1}^{\prime}+x_{2}^{\prime}\right)+ \\
& -2\left(K_{1}-K_{2}\right) \alpha_{1001} \cos \left(x_{1}^{\prime}-x_{2}^{\prime}\right)
\end{aligned}
$$

and

$$
\begin{aligned}
\nabla \eta & \cdot \nabla \psi=\frac{2 \varepsilon_{1}^{2}}{\mu_{c}^{3}} \sin 2 x_{1}^{\prime}+\frac{2 \lambda^{5} \varepsilon_{2}^{2}}{\mu_{c}^{3}} \sin 2 x_{2}^{\prime}+ \\
& +\frac{2 \varepsilon_{1} \varepsilon_{2} \lambda\left(1-\tau_{1} \tau_{2}\right)}{\mu_{c}}\left\{(\lambda+1) \sin \left(x_{1}^{\prime}+x_{2}^{\prime}\right)+(1-\lambda) \sin \left(x_{1}^{\prime}-x_{2}^{\prime}\right)\right\}+O_{o}\left(|\varepsilon|^{3}\right) .
\end{aligned}
$$

Hence we obtain the main orders of $\mathfrak{b}(\operatorname{odd}$ in $X)$ :

$$
\mathfrak{b}=-\frac{2 \varepsilon_{1}}{\mu_{c}} \sin x_{1}^{\prime}-\frac{2 \lambda^{2} \varepsilon_{2}}{\mu_{c}} \sin x_{2}^{\prime}+O_{o}\left(|\varepsilon|^{2}\right),
$$


where $O_{o}\left(|\varepsilon|^{2}\right)$ means a linear combination of odd functions $\varepsilon_{1}^{2} \sin 2 x_{1}^{\prime}, \varepsilon_{2}^{2} \sin 2 x_{2}^{\prime}$, $\varepsilon_{1} \varepsilon_{2} \sin \left(x_{1}^{\prime} \pm x_{2}^{\prime}\right)$ plus higher order terms. In using $\omega_{2}$ given by Theorem 4

$$
V_{1}=1-2 \varepsilon_{1} \cos x_{1}^{\prime}-2 \lambda \varepsilon_{2} \cos x_{2}^{\prime}-\frac{2 \varepsilon_{1}^{2}}{\mu_{c}^{2}}-\frac{2 \varepsilon_{2}^{2} \lambda^{4}}{\mu_{c}^{2}}+O_{e}^{\prime}\left(|\varepsilon|^{2}\right)
$$

$V_{2}=-2 \tau_{1} \varepsilon_{1} \cos x_{1}^{\prime}+2 \lambda \tau_{2} \varepsilon_{2} \cos x_{2}^{\prime}+\left(\beta_{1}-\frac{2 \tau_{1}}{\mu_{c}^{2}}\right) \varepsilon_{1}^{2}+\left(\beta_{2}+\frac{2 \lambda^{4} \tau_{2}}{\mu_{c}^{2}}\right) \varepsilon_{2}^{2}+O_{e}^{\prime}\left(|\varepsilon|^{2}\right)$,

where $O_{e}^{\prime}\left(|\varepsilon|^{2}\right)$ means a linear combination of even functions $\varepsilon_{1}^{2} \cos 2 x_{1}^{\prime}, \varepsilon_{2}^{2} \cos 2 x_{2}^{\prime}$, $\varepsilon_{1} \varepsilon_{2} \cos \left(x_{1}^{\prime} \pm x_{2}^{\prime}\right)$ (hence with 0 average), plus higher order terms. Hence we obtain

$$
\begin{gathered}
W_{1}=K_{1} \cdot V=1-\frac{2 \varepsilon_{1}}{\mu_{c}^{2}} \cos x_{1}^{\prime}-2 \lambda \varepsilon_{2}\left(1-\tau_{1} \tau_{2}\right) \cos x_{2}^{\prime}+ \\
+\tau_{1} \beta_{1} \varepsilon_{1}^{2}+\tau_{1} \beta_{2} \varepsilon_{2}^{2}-\frac{2 \varepsilon_{1}^{2}}{\mu_{c}^{4}}-\frac{2 \lambda^{4} \varepsilon_{2}^{2}}{\mu_{c}^{2}}\left(1-\tau_{1} \tau_{2}\right)+O_{e}^{\prime}\left(|\varepsilon|^{2}\right), \\
W_{2} \lambda^{-1}=\lambda^{-1} K_{2} \cdot V=1-2 \varepsilon_{1}\left(1-\tau_{1} \tau_{2}\right) \cos x_{1}^{\prime}-2 \lambda^{3} \varepsilon_{2} \mu_{c}^{-2} \cos x_{2}^{\prime}+ \\
-\tau_{2} \beta_{1} \varepsilon_{1}^{2}-\tau_{2} \beta_{2} \varepsilon_{2}^{2}-2 \varepsilon_{1}^{2} \mu_{c}^{-2}\left(1-\tau_{1} \tau_{2}\right)-2 \lambda^{6} \varepsilon_{2}^{2} \mu_{c}^{-4}+O_{e}^{\prime}\left(|\varepsilon|^{2}\right), \\
W_{0} \lambda^{-1}=1+\varepsilon_{1} W_{0}^{(1,0)} \cos x_{1}^{\prime}+\varepsilon_{2} W_{0}^{(0,1)} \cos x_{2}^{\prime}+ \\
+\varepsilon_{1}^{2}\left(W_{0}^{(2,0,0)}+W_{0}^{(2,0,1)} \cos 2 x_{1}^{\prime}\right)+\varepsilon_{2}^{2}\left(W_{0}^{(0,2,0)}+W_{0}^{(0,2,1)} \cos 2 x_{2}^{\prime}\right)+ \\
+\varepsilon_{1} \varepsilon_{2}\left\{W_{0}^{(1,1,+)} \cos \left(x_{1}^{\prime}+x_{2}^{\prime}\right)+W_{0}^{(1,1,-)} \cos \left(x_{1}^{\prime}-x_{2}^{\prime}\right)\right\}+O_{e}\left(|\varepsilon|^{3}\right),
\end{gathered}
$$

with

$$
\begin{aligned}
& W_{0}^{(1,0)}=2 \tau_{1}\left(\tau_{1}+\tau_{2}\right), \quad W_{0}^{(0,1)}=-2 \lambda \tau_{2}\left(\tau_{1}+\tau_{2}\right), \\
& W_{0}^{(2,0,0)}=-\left(\tau_{1}+\tau_{2}\right) \beta_{1}+4 \tau_{1} \mu_{c}^{-2}\left(\tau_{1}+\tau_{2}\right), \\
& W_{0}^{(0,2,0)}=-\left(\tau_{1}+\tau_{2}\right) \beta_{2}-2 \lambda^{6} \mu_{c}^{-4}+2 \lambda^{2}\left(1-\tau_{1} \tau_{2}\right)^{2} .
\end{aligned}
$$

We then obtain for $W_{0}$ an expansion in powers of $\varepsilon_{1}, \varepsilon_{2}$, even in $X^{\prime}$, and invariant under the symmetries $\widetilde{\mathcal{S}_{j}}$. Let us look for a formal solution of (B.4), $v_{3}^{\prime}$ being odd in $X^{\prime},\left(v_{3}^{\prime}, \rho\right)$ being invariant under the above symmetries, and in the form

$$
\begin{gathered}
v_{3}^{\prime}=\varepsilon_{1} \widetilde{u}^{(1,0)} \sin x_{1}^{\prime}+\varepsilon_{2} \widetilde{u}^{(0,1)} \sin x_{2}^{\prime}+\varepsilon_{1}^{2} \widetilde{u}^{(2,0)} \sin 2 x_{1}^{\prime}+\varepsilon_{2}^{2} \widetilde{u}^{(0,2)} \sin 2 x_{2}^{\prime}+ \\
+\varepsilon_{1} \varepsilon_{2}\left\{\widetilde{u}^{(1,1,+)} \sin \left(x_{1}^{\prime}+x_{2}^{\prime}\right)+\widetilde{u}^{(1,1,-)} \sin \left(x_{1}^{\prime}-x_{2}^{\prime}\right)\right\}+O_{o}\left(|\varepsilon|^{3}\right), \\
\rho=\lambda+\rho_{1} \varepsilon_{1}^{2}+\rho_{2} \varepsilon_{2}^{2}+O(4) .
\end{gathered}
$$

Then, in identifying the powers of $\left(\varepsilon_{1}, \varepsilon_{2}\right)$ in (B.4), for $\lambda \neq 1$ we obtain

$$
\begin{gathered}
\widetilde{u}^{(1,0)}=-\lambda W_{0}^{(1,0)}, \quad \widetilde{u}^{(0,1)}=-W_{0}^{(0,1)}, \quad \rho_{1}=\lambda W_{0}^{(2,0,0)}, \quad \widetilde{u}^{(2,0)}=-\frac{\lambda}{2} W_{0}^{(2,0,1)} \\
\widetilde{u}^{(1,1, \pm)}=-\frac{\lambda}{1 \pm \lambda}\left(W_{0}^{(1,1, \pm)}-\frac{1}{2} W_{0}^{(1,0)} W_{0}^{(0,1)}\right) \\
\rho_{2}=\lambda W_{0}^{(0,2,0)}-\frac{\lambda}{2}\left(W_{0}^{(0,1)}\right)^{2} \quad \widetilde{u}^{(0,2)}=-\frac{1}{2} W_{0}^{(0,2,1)}+\frac{1}{4}\left(W_{0}^{(0,1)}\right)^{2} .
\end{gathered}
$$

Hence, we can write

$$
\begin{aligned}
& \rho_{1}=-\lambda\left(\tau_{1}+\tau_{2}\right) \beta_{1}+4 \lambda \tau_{1} \mu_{c}^{-2}\left(\tau_{1}+\tau_{2}\right), \\
& \rho_{2}=-\lambda\left(\tau_{1}+\tau_{2}\right) \beta_{2}-4 \lambda^{5} \tau_{2} \mu_{c}^{-2}\left(\tau_{1}+\tau_{2}\right) .
\end{aligned}
$$


It is clear that when $\lambda \notin \mathbb{Q}$, the coefficients $\widetilde{u}^{(r, s, \pm)}$ of $\varepsilon_{1}^{r} \varepsilon_{2}^{s} \sin \left(r x_{1}^{\prime} \pm s x_{2}^{\prime}\right), \widetilde{u}^{(2 r, 0)}$ of $\varepsilon_{1}^{2 r} \sin 2 r x_{1}^{\prime}, \widetilde{u}^{(0,2 s)}$ of $\varepsilon_{2}^{2 s} \sin 2 s x_{2}^{\prime}, \rho_{(2 r, 0)}$ of $\varepsilon_{1}^{2 r}, \rho_{(0,2 s)}$ of $\varepsilon_{2}^{2 s}$ can be easily computed. This shows that we obtain $v_{3}^{\prime}$ and $\rho$, unique formal solution of (B.4), invariant under the symmetries $\widetilde{\mathcal{S}}_{j}$. In particular, this implies that the power series for $\rho$ only contains powers of $\left(\varepsilon_{1}^{2}, \varepsilon_{2}^{2}\right)$. In the case where $\lambda=r / s \in \mathbb{Q}$, an obstruction in the computation of $\widetilde{u}^{(r, s,-)}$ arises because of a division by 0 . In using the relations (A.14), this ends the proof of the first part of Lemma 5.

Computation of $\nu$. In order to compute $\nu$ we solve formally with respect to $v_{1}$ and $\nu$ the P.D.E. given by the first component of (B.2):

$$
K_{1} \cdot V+\nabla_{X} v_{1} \cdot V=-(\nu \mathfrak{a})^{1 / 3}\left|Y^{\prime}(X)\right|^{1 / 3}(\mathbb{Q} V \cdot V)^{1 / 6}
$$

where we notice that the unknown also occurs on the right hand side in $\left|Y^{\prime}(X)\right|$. Indeed, since $\left|Y^{\prime}(X)\right|=|\mathbb{T}|\left|D_{X^{\prime}} Y\right|$, where $D_{X^{\prime}}$ denotes the Jacobi matrix of the mapping $Y\left(X\left(X^{\prime}\right)\right)$, we have

$$
\left|Y^{\prime}(X)\right|=\operatorname{det} \mathbb{T} \operatorname{det}\left(\mathbb{I}+D_{X^{\prime}} \mathcal{V}^{\prime}\right)=-\lambda\left(\tau_{1}+\tau_{2}\right)\left(1+\operatorname{div}_{X^{\prime}} \mathcal{V}^{\prime}+\operatorname{det}\left(D_{X^{\prime}} \mathcal{V}^{\prime}\right)\right) .
$$

In using the notation as in (B.4), it results that

$$
\begin{aligned}
& \partial_{x_{1}^{\prime}} v_{1}^{\prime}+W_{0} \partial_{x_{2}^{\prime}} v_{1}^{\prime} \\
= & W_{1}^{-1}\left(\nu \lambda\left(\tau_{1}+\tau_{2}\right) \mathfrak{a}\right)^{1 / 3}(\mathbb{Q} V \cdot V)^{1 / 6}\left(1+\operatorname{div}_{X^{\prime}} \mathcal{V}^{\prime}+\operatorname{det}\left(D_{X^{\prime}} \mathcal{V}^{\prime}\right)\right)^{1 / 3}-1,
\end{aligned}
$$

which we solve in the same way as (B.4). We set

$$
\nu=\nu_{c}+\nu_{1} \varepsilon_{1}^{2}+\nu_{2} \varepsilon_{2}^{2}+\text { h.o.t. }
$$

From (B.5) and (B.6) we get the following representation for the function $\mathfrak{a}=$ $V \cdot \nabla \mathfrak{b}+\mu$

$$
\begin{gathered}
\mathfrak{a}=\mu_{c}-\frac{2}{\mu_{c}}\left\{\varepsilon_{1} \cos x_{1}^{\prime}+\varepsilon_{2} \lambda^{3} \cos x_{2}^{\prime}\right\}+\mathfrak{a}^{(2)}+\text { h.o.t., } \\
\mathfrak{a}^{(2)}=\varepsilon_{1}^{2}\left(\alpha_{1}+2 \mu_{c}^{-3}\right)+\varepsilon_{2}^{2}\left(\alpha_{2}+2 \lambda^{6} \mu_{c}^{-3}\right)+O_{e}^{\prime}\left(|\varepsilon|^{2}\right) .
\end{gathered}
$$

Hence $\nu_{c}=\left(\mu_{c} \lambda\left(\tau_{1}+\tau_{2}\right)\right)^{-1}$, and

$$
\begin{gathered}
\left(\nu \lambda\left(\tau_{1}+\tau_{2}\right) \mathfrak{a}\right)^{1 / 3}=1-2\left(3 \mu_{c}^{2}\right)^{-1}\left\{\varepsilon_{1} \cos x_{1}^{\prime}+\varepsilon_{2} \lambda^{3} \cos x_{2}^{\prime}\right\}+\mathfrak{a}_{1 / 3}^{(2)}, \\
\mathfrak{a}_{1 / 3}^{(2)}=\varepsilon_{1}^{2}\left(\frac{\alpha_{1}}{3 \mu_{c}}+\frac{4}{9 \mu_{c}^{4}}+\frac{\nu_{1}}{3}\right)+\varepsilon_{2}^{2}\left(\frac{\alpha_{2}}{3 \mu_{c}}+\frac{4 \lambda^{6}}{9 \mu_{c}^{4}}+\frac{\nu_{2}}{3}\right)+O_{e}^{\prime}\left(|\varepsilon|^{2}\right) .
\end{gathered}
$$

We also have

$$
\begin{gathered}
W_{1}^{-1}=1+\frac{2 \varepsilon_{1}}{\mu_{c}^{2}} \cos x_{1}^{\prime}+2 \lambda \varepsilon_{2}\left(1-\tau_{1} \tau_{2}\right) \cos x_{2}^{\prime}+W_{1}^{(2)}, \\
W_{1}^{(2)}=\left(\frac{4}{\mu_{c}^{4}}-\tau_{1} \beta_{1}\right) \varepsilon_{1}^{2}+ \\
+\left\{\left(\frac{2 \lambda^{4}}{\mu_{c}^{2}}+2 \lambda^{2}\left(1-\tau_{1} \tau_{2}\right)\right)\left(1-\tau_{1} \tau_{2}\right)-\tau_{1} \beta_{2}\right\} \varepsilon_{2}^{2}+O_{e}^{\prime}\left(|\varepsilon|^{2}\right)
\end{gathered}
$$

and

$$
(\mathbb{Q} V \cdot V)=V^{2}+\left(V \cdot \nabla_{X} \eta\right)^{2}=1-4 \varepsilon_{1} \cos x_{1}^{\prime}-4 \lambda \varepsilon_{2} \cos x_{2}^{\prime}+O_{e}^{\prime}\left(|\varepsilon|^{2}\right) .
$$


Finally

$$
\begin{aligned}
& \left(\nu \lambda\left(\tau_{1}+\tau_{2}\right) \mathfrak{a}\right)^{1 / 3}(\mathbb{Q} V \cdot V)^{1 / 6} W_{1}^{-1}=1+\frac{2}{3}\left(1+2 \tau_{1}^{2}\right) \varepsilon_{1} \cos x_{1}^{\prime}+ \\
& +\frac{2}{3} \lambda\left(1-\tau_{2}^{2}-3 \tau_{1} \tau_{2}\right) \varepsilon_{2} \cos x_{2}^{\prime}+c_{1} \varepsilon_{1}^{2}+c_{2} \varepsilon_{2}^{2}+O_{e}^{\prime}\left(|\varepsilon|^{2}\right)
\end{aligned}
$$

with

$$
\begin{aligned}
c_{1}= & \frac{\alpha_{1}}{3 \mu_{c}}-\tau_{1} \beta_{1}+\frac{\nu_{c} \nu_{1}}{3}+\frac{2}{9}\left(1+\tau_{1}^{2}\right)\left(15+17 \tau_{1}^{2}\right)-\frac{5}{9}, \\
c_{2}= & \frac{\alpha_{2}}{3 \mu_{c}}-\tau_{1} \beta_{2}+\frac{\nu_{c} \nu_{2}}{3}+\frac{2 \lambda^{2}}{9}\left(1+\tau_{2}^{2}\right)\left(3+2 \tau_{2}^{2}\right)+ \\
& +2 \lambda^{2}\left(1-\tau_{1} \tau_{2}\right)^{2}+\frac{2 \lambda^{2}}{3}\left(1+2 \tau_{2}^{2}\right)\left(1-\tau_{1} \tau_{2}\right)-\frac{5 \lambda^{2}}{9} .
\end{aligned}
$$

Let us define the expansion of the solution $v_{1}^{\prime}\left(X^{\prime}, \varepsilon\right)$ as

$$
\begin{aligned}
v_{1}^{\prime}= & \varepsilon_{1} v^{(1,0)} \sin x_{1}^{\prime}+\varepsilon_{2} v^{(0,1)} \sin x_{2}^{\prime}+\varepsilon_{1}^{2} v^{(2,0)} \sin 2 x_{1}^{\prime}+\varepsilon_{2}^{2} v^{(0,2)} \sin 2 x_{2}^{\prime}+ \\
& +\varepsilon_{1} \varepsilon_{2}\left\{v^{(1,1,+)} \sin \left(x_{1}^{\prime}+x_{2}^{\prime}\right)+v^{(1,1,-)} \sin \left(x_{1}^{\prime}-x_{2}^{\prime}\right)\right\}+O\left(|\varepsilon|^{3}\right),
\end{aligned}
$$

with unknown coefficients $v^{(i, j)}$. Then combining this expansion with expansion (B.8) for $v_{3}^{\prime}=v_{2}^{\prime}-\rho v_{1}^{\prime}$ we obtain

$$
\begin{gathered}
\operatorname{div}_{X^{\prime}} \mathcal{V}^{\prime}=\varepsilon_{1} v^{(1,0)} \cos x_{1}^{\prime}+\lambda\left\{v^{(0,1)}+2 \tau_{2}\left(\tau_{1}+\tau_{2}\right)\right\} \varepsilon_{2} \cos x_{2}^{\prime}+O_{e}^{\prime}\left(|\varepsilon|^{2}\right) \\
\operatorname{det}\left(D_{X^{\prime}} \mathcal{V}^{\prime}\right)=\kappa_{1} \varepsilon_{1} \varepsilon_{2} \cos \left(x_{1}^{\prime}+x_{2}^{\prime}\right)+\kappa_{2} \varepsilon_{1} \varepsilon_{2} \cos \left(x_{1}^{\prime}-x_{2}^{\prime}\right)+O_{e}\left(|\varepsilon|^{3}\right)
\end{gathered}
$$

and

$$
\begin{aligned}
& \left(1+\operatorname{div}_{X^{\prime}} \mathcal{V}^{\prime}+\operatorname{det}\left(D_{X^{\prime}} \mathcal{V}^{\prime}\right)\right)^{1 / 3}=1+\frac{\lambda}{3}\left\{v^{(0,1)}+2 \tau_{2}\left(\tau_{1}+\tau_{2}\right)\right\} \varepsilon_{2} \cos x_{2}^{\prime}+ \\
& \varepsilon_{1} \frac{v^{(1,0)}}{3} \cos x_{1}^{\prime}-\frac{1}{18}\left(v^{(1,0)}\right)^{2} \varepsilon_{1}^{2}-\frac{\lambda^{2}}{18}\left\{v^{(0,1)}+2 \tau_{2}\left(\tau_{1}+\tau_{2}\right)\right\}^{2} \varepsilon_{2}^{2}+O_{e}^{\prime}\left(|\varepsilon|^{2}\right) .
\end{aligned}
$$

Identifying powers of $\varepsilon_{1}, \varepsilon_{2}$ in (B.12) leads to

$$
\begin{gathered}
v_{1}^{(1,0)}=1+2 \tau_{1}^{2}, \quad v_{1}^{(0,1)}=1-2 \tau_{2} \tau_{1}, \\
c_{1}=-\frac{1}{18}\left(1+2 \tau_{1}^{2}\right)^{2}, \quad c_{2}=\frac{\lambda^{2}}{18}\left(1+2 \tau_{2}^{2}\right)^{2}-\frac{\lambda^{2}}{9}\left(1+2 \tau_{2}^{2}\right)\left(1-\tau_{2}^{2}-3 \tau_{1} \tau_{2}\right)+ \\
-\lambda^{2} \tau_{2}\left(\tau_{1}+\tau_{2}\right)\left(1-2 \tau_{1} \tau_{2}\right),
\end{gathered}
$$

hence

$$
\begin{aligned}
& \frac{\nu_{1}}{\nu_{c}}=-\frac{\alpha_{1}}{\mu_{c}}+3 \tau_{1} \beta_{1}+\frac{3}{2}-2\left(1+\tau_{1}^{2}\right)\left(5+6 \tau_{1}^{2}\right), \\
& \frac{\nu_{2}}{\nu_{c}}=-\frac{\alpha_{2}}{\mu_{c}}+3 \tau_{1} \beta_{2}+\frac{3 \lambda^{2}}{2}-2 \lambda^{2}\left(1+\tau_{2}^{2}\right)\left(5-6 \tau_{1} \tau_{2}\right),
\end{aligned}
$$

where $\alpha_{j}$ and $\beta_{j}$ are given by (A.13) and (A.14). The functions $v_{1}^{(2,0)}, v_{1}^{(0,2)}, v_{1}^{(1,1,+)}$, $v_{1}^{(1,1,-)}$ may be computed in the same way as $u^{\prime}$ in Appendix $\mathrm{B}$, and the necessary condition $\lambda \notin \mathbb{Q}$ is valid here again for being able to compute $v_{1}$ up to an arbitrary order. The same uniqueness argument as for $\left(v_{3}^{\prime}, \rho\right)$ works here for $\left(v_{1}^{\prime}, \nu\right)$ leading to unique expansions of $v_{1}^{\prime}, \nu$, solution of (B.12), and invariant under the symmetries $\widetilde{\mathcal{T}}_{j}$. It results that the expansion of $\nu$ is only with powers of $\left(\varepsilon_{1}^{2}, \varepsilon_{2}^{2}\right)$. Lemma 5 is then proved. 
Remark 11 Notice that for $\lambda$ close to 1 , we have $\tau_{1} \sim \tau_{2} \sim \tau, a_{1} \sim b_{2}, a_{2} \sim b_{1}$, $\alpha_{1} \sim \alpha_{2}, \beta_{1} \sim-\beta_{2}$, and $\rho_{1}+\rho_{2}=o(1)$ with

$$
\begin{gathered}
\rho_{1} \sim 4-8 \tau^{2}-20 \tau^{4}+\frac{32 \tau^{4}}{2-\mu_{c}}, \quad \mu_{c}=\left(1+\tau^{2}\right)^{-1 / 2}, \\
\frac{\nu_{1}}{\nu_{c}} \sim-\frac{17}{2}+2 \tau^{2}+24 \tau^{4}-\frac{32 \tau^{4}}{2-\mu_{c}}, \quad \frac{\nu_{2}}{\nu_{c}} \sim \frac{7}{2}-22 \tau^{2}-40 \tau^{4}+\frac{64 \tau^{4}}{2-\mu_{c}},
\end{gathered}
$$

which shows that the sign of $\rho_{1}$ and $\nu_{i}$ depends on $\tau$. We should notice that for $\tau_{1}=\tau_{2}$ we have a diamond lattice and when $\varepsilon_{1}^{2}=\varepsilon_{2}^{2}$ the rotation number is $\lambda=1$, as this results from [16], by symmetry arguments.

Diffeomorphism $X$. We are now able to give the principal parts of the diffeomorphism $X$. Indeed, from the form of $u^{\prime}$ obtained in Appendix B and the form of $v_{1}^{\prime}$ found above, we deduce that $\mathcal{V}(X, \varepsilon)=\left(v_{1}, v_{2}\right)$ with (expressed with $X^{\prime}=$ $\left(K_{1} \cdot X, K_{2} \cdot X\right)$ instead of $\left.X\right)$

$$
\begin{aligned}
& v_{1}^{\prime}=\left(1+2 \tau_{1}^{2}\right) \varepsilon_{1} \sin x_{1}^{\prime}+\left(1-2 \tau_{1} \tau_{2}\right) \varepsilon_{2} \sin x_{2}^{\prime}+O_{o}\left(|\varepsilon|^{2}\right), \\
& v_{2}^{\prime}=u^{\prime}+\rho v_{1}^{\prime}=\lambda\left(1-2 \tau_{1} \tau_{2}\right) \varepsilon_{1} \sin x_{1}^{\prime}+\lambda\left(1+2 \tau_{2}^{2}\right) \varepsilon_{2} \sin x_{2}^{\prime}+O_{o}\left(|\varepsilon|^{2}\right),
\end{aligned}
$$

where $O_{o}\left(|\varepsilon|^{2}\right)$ terms are linear combinations of odd functions $\varepsilon_{1}^{2} \sin 2 x_{1}^{\prime}, \varepsilon_{2}^{2} \sin 2 x_{2}^{\prime}$, $\varepsilon_{1} \varepsilon_{2} \sin \left(x_{1}^{\prime} \pm x_{2}^{\prime}\right)$ plus odd higher order terms. It results that

$$
X=\mathbb{T}^{-1} Y+\mathcal{W}(Y, \varepsilon), \quad \mathcal{W}(Y, \varepsilon)=\left(w_{1}, w_{2}\right)
$$

with

$$
\begin{aligned}
w_{1}= & -\varepsilon_{1} \sin y_{1}-\varepsilon_{2} \sin y_{2}+\varepsilon_{1}^{2} w_{1}^{(2,0)} \sin 2 y_{1}+\varepsilon_{2}^{2} w_{1}^{(0,2)} \sin 2 y_{2}+ \\
& +\varepsilon_{1} \varepsilon_{2}\left\{w_{1}^{(1,1,+)} \sin \left(y_{1}+y_{2}\right)+w_{1}^{(1,1,-)} \sin \left(y_{1}-y_{2}\right)\right\}+O\left(|\varepsilon|^{3}\right), \\
w_{2}= & -2 \tau_{1} \varepsilon_{1} \sin y_{1}+2 \tau_{2} \varepsilon_{2} \sin y_{2}+\varepsilon_{1}^{2} w_{2}^{(2,0)} \sin 2 y_{1}+\varepsilon_{2}^{2} w_{2}^{(0,2)} \sin 2 y_{2}+ \\
& +\varepsilon_{1} \varepsilon_{2}\left\{w_{2}^{(1,1,+)} \sin \left(y_{1}+y_{2}\right)+w_{2}^{(1,1,-)} \sin \left(y_{1}-y_{2}\right)\right\}+O\left(|\varepsilon|^{3}\right) .
\end{aligned}
$$

\section{Computation of the matrix $\mathbb{G}$ and coefficient $H$}

Coefficients $g_{i j}$ are defined in (5.5). We have

$$
\begin{aligned}
g_{11}= & \frac{1+\tau_{2}^{2}}{\left(\tau_{1}+\tau_{2}\right)^{2}}+\frac{2}{\tau_{1}+\tau_{2}} \partial_{y_{1}} \mathcal{W} \cdot\left(\begin{array}{c}
\tau_{2} \\
1
\end{array}\right)+\left(\partial_{y_{1}} \mathcal{W}\right)^{2}+\left(\nabla_{X} \eta \cdot \partial_{y_{1}} X\right)^{2} \\
g_{22}= & \frac{1+\tau_{1}^{2}}{\lambda^{2}\left(\tau_{1}+\tau_{2}\right)^{2}}+\frac{2}{\lambda\left(\tau_{1}+\tau_{2}\right)} \partial_{y_{2}} \mathcal{W} \cdot\left(\begin{array}{c}
\tau_{1} \\
-1
\end{array}\right)+\left(\partial_{y_{2}} \mathcal{W}\right)^{2}+\left(\nabla_{X} \eta \cdot \partial_{y_{2}} X\right)^{2} \\
g_{12}= & \frac{\tau_{1} \tau_{2}-1}{\lambda\left(\tau_{1}+\tau_{2}\right)^{2}}+\frac{1}{\tau_{1}+\tau_{2}} \partial_{y_{2}} \mathcal{W} \cdot\left(\begin{array}{c}
\tau_{2} \\
1
\end{array}\right)+\frac{1}{\lambda\left(\tau_{1}+\tau_{2}\right)} \partial_{y_{1}} \mathcal{W} \cdot\left(\begin{array}{c}
\tau_{1} \\
-1
\end{array}\right)+ \\
& +\left(\partial_{y_{1}} \mathcal{W}\right) \cdot\left(\partial_{y_{2}} \mathcal{W}\right)+\left(\nabla_{X} \eta \cdot \partial_{y_{1}} X\right)\left(\nabla_{X} \eta \cdot \partial_{y_{2}} X\right)
\end{aligned}
$$

and from Theorem 4 and the expression of $X$ in (B.15), (B.16)

$$
\nabla_{X} \eta \cdot \partial_{y_{1}} X=\frac{-2 \varepsilon_{1}}{\mu_{c}} \sin y_{1}+O_{o}\left(|\varepsilon|^{2}\right), \nabla_{X} \eta \cdot \partial_{y_{2}} X=\frac{-2 \lambda \varepsilon_{2}}{\mu_{c}} \sin y_{2}+O_{o}\left(|\varepsilon|^{2}\right) \text {. }
$$


We then obtain

$$
\begin{aligned}
& g_{11}=\frac{1+\tau_{2}^{2}}{\left(\tau_{1}+\tau_{2}\right)^{2}}-\frac{2\left(2 \tau_{1}+\tau_{2}\right)}{\tau_{1}+\tau_{2}} \varepsilon_{1} \cos y_{1}+\frac{\varepsilon_{1}^{2}}{2}\left(5+8 \tau_{1}^{2}\right)+O_{e}^{\prime}\left(|\varepsilon|^{2}\right), \\
& g_{22}=\frac{1+\tau_{1}^{2}}{\lambda^{2}\left(\tau_{1}+\tau_{2}\right)^{2}}-\frac{2\left(\tau_{1}+2 \tau_{2}\right)}{\lambda\left(\tau_{1}+\tau_{2}\right)} \varepsilon_{2} \cos y_{2}+\frac{\varepsilon_{2}^{2}}{2}\left(5+8 \tau_{2}^{2}\right)+O_{e}^{\prime}\left(|\varepsilon|^{2}\right), \\
& g_{12}=\frac{\tau_{1} \tau_{2}-1}{\lambda\left(\tau_{1}+\tau_{2}\right)^{2}}+\frac{\tau_{1}}{\lambda\left(\tau_{1}+\tau_{2}\right)} \varepsilon_{1} \cos y_{1}+\frac{\tau_{2}}{\tau_{1}+\tau_{2}} \varepsilon_{2} \cos y_{2}+O_{e}^{\prime}\left(|\varepsilon|^{2}\right),
\end{aligned}
$$

where the terms $O_{e}^{\prime}\left(|\varepsilon|^{2}\right)$ are linear combinations of even functions $\varepsilon_{1}^{2} \cos 2 y_{1}$, $\varepsilon_{2}^{2} \cos 2 y_{2}, \varepsilon_{1} \varepsilon_{2} \cos \left(y_{1} \pm y_{2}\right)$ plus higher order even terms. It results

$$
\begin{gathered}
g_{11}+2 \rho g_{12}+\rho^{2} g_{22}=1-2 \varepsilon_{1} \cos y_{1}-2 \lambda \varepsilon_{2} \cos y_{2}+ \\
+\varepsilon_{1}^{2}\left\{\frac{5}{2}+4 \tau_{1}^{2}+\frac{2 \rho_{1} \tau_{1}}{\lambda\left(\tau_{1}+\tau_{2}\right)}\right\}++\varepsilon_{2}^{2}\left\{\frac{5}{2}+4 \tau_{2}^{2}+\frac{2 \rho_{2} \tau_{1}}{\lambda\left(\tau_{1}+\tau_{2}\right)}\right\}+O_{e}^{\prime}\left(|\varepsilon|^{2}\right)
\end{gathered}
$$

From $(7.9)$, we have $H(\widetilde{\mathbb{Q}} \tilde{V} \cdot \tilde{V})^{1 / 2}=K(\mathbb{G} \varrho \cdot \varrho)^{1 / 2}$ and from

$$
\widetilde{\mathbb{Q}} \tilde{V} \cdot \widetilde{V}=1-4 \varepsilon_{1} \cos y_{1}-4 \lambda \varepsilon_{2} \cos y_{2}-2\left(1+2 \tau_{1}^{2}\right) \varepsilon_{1}^{2}-2 \lambda^{2}\left(1+2 \tau_{2}^{2}\right) \varepsilon_{2}^{2}+O_{e}^{\prime}\left(|\varepsilon|^{2}\right),
$$

and (C.1), we obtain

$$
\begin{aligned}
\frac{\mathbb{G} \underline{\varrho} \cdot \underline{\varrho}}{\widetilde{\mathbb{Q}} \widetilde{V} \cdot \widetilde{V}}= & 1+2 \varepsilon_{1} \cos y_{1}+2 \lambda \varepsilon_{2} \cos y_{2}+\left(\frac{17}{2}+8 \tau_{1}^{2}+\frac{2 \rho_{1} \tau_{1}}{\lambda\left(\tau_{1}+\tau_{2}\right)}\right) \varepsilon_{1}^{2}+ \\
& +\left(\frac{5}{2}+6 \lambda^{2}+4 \tau_{2}^{2}\left(1+\lambda^{2}\right)+\frac{2 \rho_{2} \tau_{1}}{\lambda\left(\tau_{1}+\tau_{2}\right)}\right) \varepsilon_{2}^{2}+O_{e}^{\prime}\left(|\varepsilon|^{2}\right) .
\end{aligned}
$$

From (7.16) and the decomposition (6.3) we have $K=1+O_{e}\left(|\varepsilon|^{2 m+1}\right)$, hence

$$
\begin{aligned}
H= & 1+\varepsilon_{1} \cos y_{1}+\lambda \varepsilon_{2} \cos y_{2}+\left(4\left(1+\tau_{1}^{2}\right)+\frac{\rho_{1} \tau_{1}}{\lambda\left(\tau_{1}+\tau_{2}\right)}\right) \varepsilon_{1}^{2}+ \\
& +\left(\frac{5}{4}+\frac{11}{4} \lambda^{2}+2 \tau_{2}^{2}\left(1+\lambda^{2}\right)+\frac{\rho_{2} \tau_{1}}{\lambda\left(\tau_{1}+\tau_{2}\right)}\right) \varepsilon_{2}^{2}+O_{e}^{\prime}\left(|\varepsilon|^{2}\right),
\end{aligned}
$$

i.e. from (B.9), (B.10)

$$
\begin{aligned}
H= & 1+\varepsilon_{1} \cos y_{1}+\lambda \varepsilon_{2} \cos y_{2}+\left(4\left(1+\tau_{1}^{2}\right)^{2}-\tau_{1} \beta_{1}\right) \varepsilon_{1}^{2}+ \\
& +\left(4 \lambda^{2}\left(1+\tau_{2}^{2}\right)\left(1-\tau_{1} \tau_{2}\right)+\left(1-\lambda^{2}\right)\left(2 \tau_{2}^{2}+\frac{5}{4}\right)-\tau_{1} \beta_{2}\right) \varepsilon_{2}^{2}+O_{e}^{\prime}\left(|\varepsilon|^{2}\right)
\end{aligned}
$$

\section{Proof of Theorem 5}

We split the proof into two parts and begin with the calculation of the variation of $\mathcal{F}_{3}$. It directly acts on the mapping $X(Y)$, and depends implicitly on functions $\psi$, $\eta$, parameters $\nu, \mu, \rho$, and on the unit vector $\mathbf{u}$. Our goal is to deduce the expression for the Gateaux derivative $D_{(U, X, \mu, \mathbf{u})} \mathcal{F}_{3}$. The result is given by the following

Lemma 19 Under the assumptions of Theorem 5,

$$
\begin{array}{r}
D_{(U, X, \mu, \mathbf{u})} \mathcal{F}_{3}[\delta U, \delta X, \delta \mu, \delta \mathbf{u}]=X^{\prime}\left(\varrho \cdot \nabla v-\frac{1}{3} \operatorname{div} v \varrho\right)- \\
H \mathbf{V}[\delta U, \delta \mathbf{u}+\nabla \delta \phi]+X^{\prime}(\mathbf{S}[\delta U, \delta \mathbf{u}, \delta \mu] \varrho)+\mathcal{R}_{3}
\end{array}
$$


with the remainder

$$
\mathcal{R}_{3}=v \cdot \nabla \mathcal{F}_{3}+\frac{1}{3} \operatorname{div} v \mathcal{F}_{3}-\mathbf{S}[\delta U, \delta \mathbf{u}, \delta \mu] \mathcal{F}_{3},
$$

and the (2-components) linear form $\mathbf{S}$ is defined by the equality

$$
\begin{aligned}
\mathbf{S}= & \frac{1}{3}\left((\delta \mu+\mathbf{N}(\delta \mathbf{u}+\nabla \delta \phi)+\mathbf{M}(\delta \eta)) \frac{1}{\mathfrak{a}}+\right. \\
& \left.+(V \cdot(\delta \mathbf{u}+\nabla \delta \phi)+(\mathfrak{a}-\mu) \delta \eta) \frac{1}{\mathbb{Q} V \cdot V}\right)
\end{aligned}
$$

in which the linear forms $\mathbf{N}$ and $\mathbf{M}$ are defined by

$$
\begin{aligned}
\mathbf{N}(\boldsymbol{\xi}) & =V \cdot \nabla\left\{\frac{1}{1+|\nabla \eta|^{2}} \boldsymbol{\xi} \cdot \nabla \eta\right\}+\mathbb{Q}^{-1} \nabla \mathfrak{b} \cdot \boldsymbol{\xi}, \\
\mathbf{M}(\delta \eta) & =V \cdot \nabla\left\{\frac{1}{1+|\nabla \eta|^{2}}[\delta \eta(\nabla \mathfrak{b} \cdot \nabla \eta)+V \cdot \nabla \delta \eta]\right\}+ \\
& +\mathbb{Q}^{-1} \nabla \mathfrak{b} \cdot(\delta \eta \nabla \mathfrak{b}-(V \cdot \nabla \delta \eta) \nabla \eta) .
\end{aligned}
$$

The linear form $\mathbf{V}$ is given by the formula

$$
\mathbf{V}[\delta \eta, \delta \mathbf{u}+\nabla \delta \phi]=\mathbb{Q}^{-1}(\delta \mathbf{u}+\nabla \delta \phi)+\mathbb{Q}^{-1}(-(V \cdot \nabla \delta \eta) \nabla \eta+\delta \eta \nabla \mathfrak{b}) .
$$

The matrix $\mathbb{Q}$ is defined in (5.5) and function $H$ by (7.9).

Proof By abuse of notation, further we simply write $\delta F$ instead of $D_{(U, \mu, \mathbf{u})} F[\delta U, \delta \mu, \delta \mathbf{u}]$. We set in what follows

$$
H_{0}=-(\nu \mathfrak{a})^{-1 / 3}\left(V^{2}+\left(V \cdot \nabla_{X} \eta\right)^{2}\right)^{-1 / 6} \equiv\left|X^{\prime}\right|^{-1 / 3} H .
$$

It follows from $\mathbb{Q} V=\mathbf{u}+\nabla_{X} \psi$ and (5.5) that we have

$$
\mathbb{Q} \delta V=\delta \mathbf{u}+\nabla \delta \psi-\delta \mathbb{Q} V=\delta \mathbf{u}+\nabla \delta \psi-(V \cdot \nabla \eta) \nabla \delta \eta-(V \cdot \nabla \delta \eta) \nabla \eta .
$$

Recalling the relation $\delta \psi=\delta \phi+(V \cdot \nabla \eta) \delta \eta$ we get

$$
\delta V=\mathbb{Q}^{-1}(\delta \mathbf{u}+\nabla \delta \phi-(V \cdot \nabla \delta \eta) \nabla \eta+\delta \eta \nabla \mathfrak{b}) .
$$

Next we have $V^{2}+(V \cdot \nabla \eta)^{2}=\mathbb{Q} V \cdot V$, which yields

$$
\begin{aligned}
& \delta\left(V^{2}+(V \cdot \nabla \eta)^{2}\right)=2 \mathbb{Q} \delta V \cdot V+\delta \mathbb{Q} V \cdot V= \\
& 2 \delta \mathbf{u} \cdot V+2 \nabla \delta \phi \cdot V+2 \delta \eta(V \cdot \nabla \mathfrak{b}) .
\end{aligned}
$$

Recalling the notation of the function $\mathfrak{a}$ we conclude from this that

$$
\delta(\mathbb{Q} V \cdot V)=2(V \cdot \nabla \delta \phi+(\mathfrak{a}-\mu) \delta \eta)+2 \delta \mathbf{u} \cdot V .
$$

deltaGee

Next we have to calculate the variation of the coefficient $\mathfrak{a}$. Note that

$$
\begin{aligned}
& \delta \mathfrak{b}=\delta V \cdot \nabla \eta+V \cdot \nabla \delta \eta= \\
& \quad(\delta \mathbf{u}+\nabla \delta \phi-(V \cdot \nabla \delta \eta) \nabla \eta+\delta \eta \nabla \mathfrak{b}) \cdot \mathbb{Q}^{-1} \nabla \eta+V \cdot \nabla \delta \eta .
\end{aligned}
$$

Since $\mathbb{Q}^{-1} \nabla \eta=\left(1+|\nabla \eta|^{2}\right)^{-1} \nabla \eta$, we conclude from this that

$$
\delta \mathfrak{b}=\left(1+|\nabla \eta|^{2}\right)^{-1}((\delta \mathbf{u}+\nabla \delta \phi+\delta \eta \nabla \mathfrak{b}) \cdot \nabla \eta+V \cdot \nabla \delta \eta) .
$$


From this and the equality $\delta \mathfrak{a}=\delta V \cdot \nabla \mathfrak{b}+V \cdot \nabla \delta \mathfrak{b}+\delta \mu$ we obtain

$$
\delta \mathfrak{a}=\mathbf{N}(\delta \mathbf{u}+\nabla \delta \phi)+\mathbf{M}(\delta \eta)+\delta \mu,
$$

where the linear form $\mathbf{N}$ and linear differential form $\mathbf{M}$ are defined by (D.3). Next we have

$$
\delta H_{0}=-\frac{H_{0}}{3}\left(\frac{\delta \mathfrak{a}}{\mathfrak{a}}+\frac{\delta(\mathbb{Q} V \cdot V)}{2(\mathbb{Q} V \cdot V)}\right),
$$

and by substituting (D.7) and (D.8), this leads to the identity

$$
\begin{aligned}
\delta H_{0} & =-\frac{H_{0}}{3}\left((\delta \mu+\mathbf{N}(\delta \mathbf{u}+\nabla \delta \phi)+\mathbf{M}(\delta \eta)) \frac{1}{\mathfrak{a}}+\right. \\
& \left.+(V \cdot(\delta \mathbf{u}+\nabla \delta \phi)+(\mathfrak{a}-\mu) \delta \eta) \frac{1}{\mathbb{Q} V \cdot V}\right)=-H_{0} \mathbf{S}[\delta U, \delta \mathbf{u}, \delta \mu] .
\end{aligned}
$$

Next we have

$$
\begin{aligned}
\delta \mathbf{F} & =\delta H_{0} V+H_{0} \delta V=\left(\left|X^{\prime}\right|^{-1 / 3} H_{0}^{-1} \delta H_{0}\right)\left|X^{\prime}\right|^{1 / 3} \mathbf{F}+H_{0} \delta V \\
& =\left(\left|X^{\prime}\right|^{-1 / 3} H_{0}^{-1} \delta H_{0}\right)\left(\mathcal{D} X-\mathcal{F}_{3}\right)+H_{0} \delta V,
\end{aligned}
$$

thus we get

$$
\delta \mathbf{F}=-\left|X^{\prime}\right|^{-1 / 3} \mathbf{S}[\delta U, \delta \mu, \delta \mathbf{u}]\left(\mathcal{D} X-\mathcal{F}_{3}\right)+H_{0} \delta V .
$$

Finally note that

$$
D_{(U, X, \mu, \mathbf{u})} \mathcal{F}_{3}[\delta U, \delta X, \delta \mu, \delta \mathbf{u}]=D_{X} \mathcal{F}_{3}[\delta X]-\left|X^{\prime}\right|^{1 / 3} D_{(U, \mu, \mathbf{u})} \mathbf{F}[\delta U, \delta \mu, \delta \mathbf{u}] .
$$

It follows from (5.10) that the operator $X \mapsto \mathcal{F}_{3}(X)$ meets all requirements of Lemma 7 with $d=2, \varrho=(1, \rho)$, and $\alpha=1 / 3$. Thus we get for $\delta X=X^{\prime} v$,

$$
\partial_{X} \mathcal{F}_{3}\left[X^{\prime} v\right]=X^{\prime}\left(\mathcal{D} v-\frac{1}{3}(\operatorname{div} v) \varrho\right)+v \cdot \nabla \mathcal{F}_{3}+\frac{1}{3}(\operatorname{div} v) \mathcal{F}_{3}
$$

linearization $F_{-} 3 a$

Combining (D.11), (D.12), and the identity $\mathbf{V}[\delta \eta, \delta \mathbf{u}+\nabla \delta \phi]=\delta V$ we obtain (D.1), which completes the proof.

Proof of Theorem 5. By virtue of (4.2), (4.4) and Lemma 19 this relation can be written in the form

$$
\begin{gathered}
\mathcal{G}_{\eta} \delta \phi-\operatorname{div}{ }_{X}(\delta \eta V)-\nabla_{X} \eta \cdot \delta \mathbf{u}=\delta \mathcal{F}_{1}-\mathcal{R}_{1}[\delta U], \\
V \cdot \nabla_{X} \delta \phi+\mathfrak{a} \delta \eta+\left(\nabla_{X} \psi-\mathfrak{b} \nabla \eta\right) \cdot \delta \mathbf{u}+\eta \delta \mu=\delta \mathcal{F}_{2}, \\
\mathcal{D} v-\frac{1}{3}(\operatorname{div} v) \varrho+\mathbf{S}[\delta U, \delta \mu, \delta \mathbf{u}] \varrho-X^{\prime-1} H \mathbf{V}[\delta \eta, \delta \mathbf{u}+\nabla \delta \phi]= \\
X^{\prime-1} \delta \mathcal{F}_{3}-X^{\prime-1} \mathcal{R}_{3}[\delta U, \delta X, \delta \mu, \delta \mathbf{u}] .
\end{gathered}
$$

linearizedfe2

Note that $X$ is the independent variable in equations (D.13), and $Y$ is the independent variable in (D.14). In order to take advantage of the special choice of the diffeomorphism $X(Y)$, it is convenient to change the independent variable $X$ into $Y$ in equations (D.13). To this end we set

$$
u(Y)=\widetilde{\delta \phi}(Y), \quad \varsigma(Y)=\widetilde{\delta \eta}(Y), \quad \overline{\mathcal{G}} u(Y)=\widetilde{\mathcal{G} \eta} \delta \phi(Y) .
$$

It follows from (5.10) that

$$
V \cdot \nabla_{X} \delta \phi \equiv\left(X^{\prime-1} V\right) \cdot \nabla_{Y} u=\frac{1}{H}\left(\varrho-X^{\prime-1} \mathcal{F}_{3}\right) \cdot \nabla_{Y} u .
$$


Using this relation we can rewrite equations (D.13) in the equivalent form (7.6)(7.7). It remains to prove (7.8). We begin with the change of variables in the linear forms $\mathbf{M}, \mathbf{N}$ and $\mathbf{V}$. Recalling equality (5.5) and expressions (D.3) we obtain

$$
\mathbf{N}\left[\left(X^{\prime *}\right)^{-1} \boldsymbol{\xi}\right]=\left(X^{\prime}(Y)^{-1} \widetilde{V}\right) \cdot \nabla_{Y}\left(\frac{1}{1+\mathbf{q}_{2}^{2}} X^{\prime-1} \mathbf{q}_{2} \cdot \boldsymbol{\xi}\right)+\mathbb{G}^{-1} \nabla \widetilde{\mathfrak{b}} \cdot \boldsymbol{\xi}
$$

Using the identity

$$
X^{\prime}(Y)^{-1} \widetilde{V}=\frac{1}{H} \varrho-\frac{1}{H} X^{\prime}(Y)^{-1} \mathcal{F}_{3}
$$

we can rewrite this relation in the form

$$
\mathbf{N}\left[\left(X^{\prime *}\right)^{-1} \boldsymbol{\xi}\right]=\overline{\mathbf{N}}[\boldsymbol{\xi}]+\mathcal{R}_{N}[\boldsymbol{\xi}],
$$

where $\overline{\mathbf{N}}$ and $\mathcal{R}_{N}$ are defined by (7.11) and (7.12). Arguing as before we obtain from (D.3)

$$
\mathbf{M}[\delta \eta]=\overline{\mathbf{M}}[\varsigma]+\mathcal{R}_{M}[\varsigma],
$$

where $\overline{\mathbf{M}}$ and $\mathcal{R}_{N}$ are defined by (7.11) and (7.14). Finally repeating these arguments, gives the expression for the linear form $\mathbf{V}$,

$$
H X^{\prime-1} \mathbf{V}[\delta \eta, \delta \mathbf{u}+\nabla \delta \phi]=\overline{\mathbf{V}}[\boldsymbol{\xi}, \varsigma]+\mathcal{R}_{V}[\varsigma],
$$

where

$$
\begin{gathered}
\boldsymbol{\xi}=X^{\prime *}\left(\delta \mathbf{u}+\nabla_{X} \delta \phi\right)=\left(X^{\prime *} \delta \mathbf{u}+\nabla u\right), \\
\overline{\mathbf{V}}[\boldsymbol{\xi}, \varsigma]=H \mathbb{G}^{-1} \boldsymbol{\xi}-(\mathcal{D} \varsigma) \mathbb{G}^{-1} \nabla \widetilde{\eta}+\varsigma H \mathbb{G}^{-1} \nabla \widetilde{\mathfrak{b}},
\end{gathered}
$$

and $\mathcal{R}_{V}$ is given by (7.15). Now we can change the variables in the linear form $\mathbf{S}$ To this end note that

$$
\mathbf{S}[\delta U, \delta \mathbf{u}, \delta \mu]=\mathbf{S}[(\delta \phi, 0), \delta \mathbf{u}, 0]+\mathbf{S}[(\mathfrak{b} \delta \eta, \delta \eta), 0,0]+\mathbf{S}[0,0, \delta \mu] .
$$

Using representations (D.15) and identity (D.17) we get

$$
\mathbf{S}[(\delta \phi, 0), \delta \mathbf{u}, 0]=\frac{1}{3 \widetilde{\mathfrak{a}}} \overline{\mathbf{N}}[\boldsymbol{\xi}]+\frac{1}{3 H \widetilde{\mathbb{Q}} \widetilde{V} \cdot \widetilde{V}}\left(\varrho \cdot \boldsymbol{\xi}-X^{\prime-1} \mathcal{F}_{3} \cdot \boldsymbol{\xi}\right)+\frac{1}{3 \widetilde{\mathfrak{a}}} \mathcal{R}_{N}[\boldsymbol{\xi}]
$$

Next we have from (D.19)

$$
\mathbf{S}[(\mathfrak{b} \delta \eta, \delta \eta), 0,0]=\frac{1}{3 \widetilde{\mathfrak{a}}} \overline{\mathbf{M}}[\varsigma]+\left[\frac{\widetilde{\mathfrak{a}}-\mu}{3 \widetilde{\mathbb{Q}} \widetilde{V} \cdot \widetilde{V}}\right] \varsigma+\frac{1}{3 \widetilde{\mathfrak{a}}} \mathcal{R}_{M}[\varsigma]
$$

and

$$
\mathbf{S}[0,0, \delta \mu]=\frac{1}{3 \widetilde{\mathfrak{a}}} \delta \mu .
$$

Combining (D.24), (D.25), and (D.26) we finally obtain the expression for $\mathbf{S}[\delta U, \delta \mathbf{u}, \delta \mu]$ in terms of $u$ and $\varsigma$ :

$$
\frac{1}{3 \widetilde{\mathfrak{a}}}(\delta \mu+\overline{\mathbf{N}}[\boldsymbol{\xi}]+\overline{\mathbf{M}}[\varsigma])+\frac{1}{3 H(\widetilde{\mathbb{Q}} \widetilde{V} \cdot \widetilde{V})} \varrho \cdot \boldsymbol{\xi}+\left[\frac{\widetilde{\mathfrak{a}}-\mu}{3 \widetilde{\mathbb{Q}} \widetilde{V} \cdot \widetilde{V}}\right] \varsigma+\mathcal{R}_{S}[\boldsymbol{\xi}, \varsigma],
$$

where $\boldsymbol{\xi}$ and $R_{S}$ are given by (D.21) and (7.12). Combining this result with (D.19), (D.22) and recalling the notation in Theorem 5 we obtain

$$
\begin{aligned}
& \mathbf{S}[\delta U, \delta \mathbf{u}, \delta \mu] \varrho-H X^{\prime-1} \mathbf{V}[\delta \eta, \delta \mathbf{u}+\nabla \delta \phi]=\mathbf{T}\left[X^{\prime *} \delta \mathbf{u}+\nabla u, \varsigma\right]+ \\
& \frac{1}{3 \widetilde{\mathfrak{a}}} \delta \mu \varrho+\mathcal{R}_{S}\left[X^{\prime *} \delta \mathbf{u}+\nabla u, \varsigma\right] \boldsymbol{\varrho}-\mathcal{R}_{V}[\varsigma]
\end{aligned}
$$

Substituting this expression into (D.14) completes the proof of Theorem 5 . 


\section{E Proof of Lemma 10}

Using the definition (8.25) of the resonance set $\mathcal{N}$, we first prove that the inequalities

$$
\left|p_{1}+\rho p_{2}\right| \leq \frac{3}{4 \sqrt{\nu}}\left|\mathbb{A}^{1 / 2} \tilde{\mathbf{p}}\right|^{1 / 2} \text { and } L(\mathbf{p}) \geq \frac{7}{16}\left|\mathbb{A}^{1 / 2} \tilde{\mathbf{p}}\right|
$$

hold true for all $\mathbf{p} \in \mathbb{Z}^{2} \backslash\{0\}$ with the following property. There exists $\mathbf{k} \in \mathbb{Z}^{2} \backslash\{0\}$ such that

$$
|\mathbf{p}-\mathbf{k}| \leq c_{2}\left|\mathbb{A}^{1 / 2} \tilde{\mathbf{p}}\right|^{1 / 2},
$$

$$
\mathbf{k} \in \mathbb{Z}^{2} \backslash \mathcal{N} \text { when } \mathbf{p} \in \mathcal{N}, \quad \text { and } \mathbf{k} \in \mathcal{N} \text { when } \mathbf{p} \in \mathbb{Z}^{2} \backslash \mathcal{N} \text {. }
$$

The constant $c_{2}$ independent on $\mathbf{p}, \mathbf{k}$ will be specified below. To do this we note that by virtue of (E.3), the continuous function

$$
2 \nu^{1 / 2}\left|x_{1}+\rho x_{2}\right|-\left|\mathbb{A}^{1 / 2} \tilde{\mathbf{x}}\right|^{1 / 2} \text { where } \tilde{\mathbf{x}}=\left(x_{1}+\rho x_{2}, x_{2}\right)
$$

changes its sign when a point $\mathbf{x}$ runs along the segment $[\mathbf{p}, \mathbf{k}]$. Hence there exists $\mathbf{x} \in[\mathbf{p}, \mathbf{k}]$ such that

$$
2 \nu^{1 / 2}\left|x_{1}+\rho x_{2}\right|=\left|\mathbb{A}^{1 / 2} \tilde{\mathbf{x}}\right|^{1 / 2} .
$$

Now assume that $|\mathbf{p}-\mathbf{k}| \leq \delta$, where $\delta$ will be specified below. We have $|\mathbf{p}-\mathbf{x}| \leq$ $|\mathbf{p}-\mathbf{k}| \leq \delta$, hence

$$
\left|p_{1}+\rho p_{2}\right| \leq\left|x_{1}+\rho x_{2}\right|+\left|\left(p_{1}-x_{1}\right)+\rho\left(p_{2}-x_{2}\right)\right| \leq\left|x_{1}+\rho x_{2}\right|+(1+\rho) \delta .
$$

Because of the choice of $\mathbf{x}$ we have

$$
2 \nu^{1 / 2}\left|p_{1}+\rho p_{2}\right| \leq\left|\mathbb{A}^{1 / 2} \tilde{\mathbf{x}}\right|^{1 / 2}+2 \nu^{1 / 2}(1+\rho) \delta .
$$

On the other hand, we have

$$
\left|\mathbb{A}^{1 / 2} \tilde{\mathbf{x}}\right|^{1 / 2} \leq\left|\mathbb{A}^{1 / 2} \tilde{\mathbf{p}}\right|^{1 / 2}+\left|\mathbb{A}^{1 / 2}(\tilde{\mathbf{x}}-\tilde{\mathbf{p}})\right|^{1 / 2} \leq\left|\mathbb{A}^{1 / 2} \tilde{\mathbf{p}}\right|^{1 / 2}+\|\mathbb{A}\|^{1 / 4}(1+\rho)^{1 / 2} \delta^{1 / 2},
$$

which leads to the inequality

$$
2 \nu^{1 / 2}\left|p_{1}+\rho p_{2}\right| \leq\left|\mathbb{A}^{1 / 2} \tilde{\mathbf{p}}\right|^{1 / 2}+2 \nu^{1 / 2}(1+\rho) \delta+\|\mathbb{A}\|^{1 / 4}(1+\rho)^{1 / 2} \sqrt{\delta} .
$$

Now choose $\delta>0$ so that

$$
(1+\rho) \delta+\frac{1}{2 \sqrt{\nu}}\|\mathbb{A}\|^{1 / 4}(1+\rho)^{1 / 2} \sqrt{\delta} \leq \frac{1}{4 \sqrt{\nu}}\left|\mathbb{A}^{1 / 2} \tilde{\mathbf{p}}\right|^{1 / 2} .
$$

Note that $\sqrt{\delta} \in\left[z_{1}, z_{2}\right]$, where $z_{i}$ are the roots of the polynomial

$$
z^{2}+b z-d=0, \quad b=\frac{1}{2(1+\rho)^{1 / 2} \sqrt{\nu}}\|\mathbb{A}\|^{1 / 4}, \quad d=\frac{1}{4(1+\rho) \sqrt{\nu}}\left|\mathbb{A}^{1 / 2} \tilde{\mathbf{p}}\right|^{1 / 2} .
$$

Since $z_{1}<0$ we can choose any $\delta$ satisfying the inequality

$$
0<\sqrt{\delta} \leq z_{2}=(2 b)^{-1}\left(\sqrt{1+4 d / b^{2}}-1\right) .
$$

Next note that since $\mathbf{p}$ is an integer $\neq 0$ (because we consider operators in the space of odd functions which are invariant with respect to the action of $\mathfrak{H}$ and $\Pi$ ) we have $\left|\mathbb{A}^{1 / 2} \tilde{\mathbf{p}}\right| \geq A_{0}>0$, hence

$$
4 d / b^{2} \geq 4 \sqrt{\nu A_{0} /\|\mathbb{A}\|}:=c_{0} \text { independent on } \mathbf{p} .
$$

Noting that for all $z \geq c_{0}$,

$$
\sqrt{1+z}-1 \geq c_{1} \sqrt{z} \text { with } c_{1}=\sqrt{1+c_{0}^{-1}}-c_{0}^{-1}
$$


we obtain from this that

$$
z_{2} \geq 2^{-1} b c_{1} \sqrt{4 d / b^{2}}=c_{1} \sqrt{d} .
$$

From this and the expression for $d$ we conclude that (E.4) is fulfilled for all $\delta$ satisfying the inequality

$$
\delta \leq c_{1}^{2} 4^{-1}(1+\rho)^{-1} \nu^{-1 / 2}\left|\mathbb{A}^{1 / 2} \tilde{\mathbf{p}}\right|^{1 / 2}=c_{2}\left|\mathbb{A}^{1 / 2} \tilde{\mathbf{p}}\right|^{1 / 2},
$$

which completes the proof of (E.1).

For each $\mathbf{p} \in \mathbb{Z}^{2} \backslash\{0\}$ we denote by $\mathcal{K}(\mathbf{p}) \subset \mathbb{Z}^{2} \backslash\{0\}$, the set of all points $\mathbf{k}$ satisfying the inequality (E.2). Now define the operators $\mathfrak{Y}$ and $\mathfrak{Z}$ by the equalities

$$
\begin{aligned}
& (\widehat{\mathfrak{Y} u})_{\mathbf{p}}=\frac{1}{L(\mathbf{p})} \sum_{\mathbf{k} \in \mathcal{K}(\mathbf{p})} \widehat{H}(\mathbf{p}-\mathbf{k}, \boldsymbol{\xi}(\mathbf{k}))\left(\chi_{\mathcal{N}}(\mathbf{p})-\chi_{\mathcal{N}}(\mathbf{k})\right) \widehat{u}_{\mathbf{k}}, \quad \mathbf{p} \neq 0, \\
& (\widehat{\mathfrak{Z} u})_{\mathbf{p}}=\sum_{\mathbf{k} \in \mathbb{Z}^{2} \backslash \mathcal{K}(\mathbf{p}) \cup\{0\}} \widehat{H}(\mathbf{p}-\mathbf{k}, \boldsymbol{\xi}(\mathbf{k}))\left(\chi_{\mathcal{N}}(\mathbf{p})-\chi_{\mathcal{N}}(\mathbf{k})\right) \widehat{u}_{\mathbf{k}}, \quad \mathbf{p} \neq 0,
\end{aligned}
$$

where $\chi_{\mathcal{N}}$ is a characteristic function of the resonance set $\mathcal{N}$. It is clear that $\Pi \mathfrak{H}-$ $\mathfrak{H} \Pi=\mathfrak{Y} \mathfrak{L}+\mathfrak{Z}$. Let us estimate the norms of operators $\mathfrak{Y}$ and $\mathfrak{Z}$. By hypothesis on $\mathfrak{H}$ we have

$$
|\widehat{H}(\mathbf{p}-\mathbf{k}, \boldsymbol{\xi}(\mathbf{k}))| \leq c \varepsilon(1+|\mathbf{p}-\mathbf{k}|)^{-r}
$$

hence

$$
(1+|\mathbf{p}|)^{s+1}\left|(\widehat{\mathfrak{Y} u})_{\mathbf{p}}\right| \leq c \varepsilon \frac{(1+|\mathbf{p}|)^{s+1}}{|L(\mathbf{p})|} \sum_{\mathbf{k} \in \mathcal{K}(\mathbf{p})}(1+|\mathbf{p}-\mathbf{k}|)^{-r}\left|\chi_{\mathcal{N}}(\mathbf{p})-\chi_{\mathcal{N}}(\mathbf{k})\right|\left|\widehat{u}_{\mathbf{k}}\right| .
$$

Note that $\chi_{N}(\mathbf{p}) \neq \chi_{N}(\mathbf{k})$ if and only if $\mathbf{k}$ satisfies (E.3). It follows from this and the definition of $\mathcal{K}(\mathbf{p})$ that we can apply (E.1) to obtain

$$
(1+|\mathbf{p}|) \frac{1}{|L(\mathbf{p})|}\left|\chi_{\mathcal{N}}(\mathbf{p})-\chi_{\mathcal{N}}(\mathbf{k})\right| \leq c \text { for } \mathbf{k} \in \mathcal{K}(\mathbf{p}),
$$

which gives for any integer $t \geq 0$

$$
\frac{(1+|\mathbf{p}|)^{t+1}}{|L(\mathbf{p})|}\left|\chi_{\mathcal{N}}(\mathbf{p})-\chi_{\mathcal{N}}(\mathbf{k})\right| \leq c(1+|\mathbf{p}-\mathbf{k}|)^{t}+c(1+|\mathbf{k}|)^{t} \text { for } \mathbf{k} \in \mathcal{K}(\mathbf{p}),
$$

where $c$ is a generic constant only depending on $t$. This yields

$$
(1+|\mathbf{p}|)^{s+1}\left|(\widehat{\mathfrak{Y} u})_{\mathbf{p}}\right| \leq c \varepsilon \sum_{\mathbf{k} \in \mathcal{K}(\mathbf{p})}\left((1+|\mathbf{p}-\mathbf{k}|)^{-r}(1+|\mathbf{k}|)^{s}+(1+|\mathbf{p}-\mathbf{k}|)^{-r+s}\right)\left|\widehat{u}_{\mathbf{k}}\right|
$$

for $\mathbf{p} \neq \mathbf{0}$. By using Cauchy-Schwarz inequality on each sum on the right hand side, we are faced with estimating the sum

$$
\sum_{\mathbf{p} \in \mathbb{Z}^{2} \backslash\{0\}} \sum_{\mathbf{k} \in \mathcal{K}(\mathbf{p})} \frac{1}{(1+|\mathbf{p}-\mathbf{k}|)^{2(r-s)}} .
$$

By using a comparison with the integral

$$
\int_{\mathbf{x} \in \mathbb{R}^{2}} \int_{|\mathbf{x}-\mathbf{y}|<c(1+|\mathbf{x}|)^{1 / 2}} \frac{d x_{1} d x_{2} d y_{1} d y_{2}}{(1+|\mathbf{x}-\mathbf{y}|)^{2(r-s)}} \leq c^{\prime} \int_{\mathbf{x} \in \mathbb{R}^{2}} \frac{d x_{1} d x_{2}}{(1+|\mathbf{x}|)^{(r-s-1)}}
$$

we conclude that for $0 \leq s<r-3$,

$$
\sum_{\mathbf{p} \in \mathbb{Z}^{2} \backslash\{0\}}(1+|\mathbf{p}|)^{2 s+2}\left|(\widehat{\mathcal{Y} u})_{\mathbf{p}}\right|^{2} \leq c \varepsilon^{2} \sum_{\mathbf{k} \in \mathbb{Z}^{2} \backslash\{0\}}(1+|\mathbf{k}|)^{2 s}\left|\widehat{u}_{\mathbf{k}}\right|^{2},
$$


which yields the desired estimate (8.28) for $\mathfrak{Y}$. In order to estimate $\mathfrak{Z}$ we note that

$$
\left.\left|(\widehat{\mathfrak{Z} u})_{\mathbf{p}}\right| \leq c \varepsilon \sum_{\mathbf{k} \in \mathbb{Z}^{2} \backslash \mathcal{K}(\mathbf{p}) \cup\{0\}}(1+|\mathbf{p}-\mathbf{k}|)^{-r} \mid\right)\left|\widehat{u}_{\mathbf{k}}\right| .
$$

Since for $\mathbf{k} \in \mathbb{Z}^{2} \backslash \mathcal{K}(\mathbf{p}) \cup\{0\}$ and $\mathbf{p} \neq 0$,

$$
|\mathbf{p}-\mathbf{k}| \geq c_{2}\left|\mathbb{A}^{1 / 2} \tilde{\mathbf{p}}\right|^{1 / 2} \geq c(1+|\mathbf{p}|)^{1 / 2}
$$

and using again $(1+|\mathbf{p}|)^{t} \leq c(1+|\mathbf{p}-\mathbf{k}|)^{t}+c(1+|\mathbf{k}|)^{t}$, we have

$$
(1+|\mathbf{p}|)^{s+1}\left|(\widehat{\mathfrak{Z} u})_{\mathbf{p}}\right| \leq c \varepsilon \sum_{\mathbf{k} \in \mathbb{Z}^{2} \backslash \mathcal{K}(\mathbf{p})}\left((1+|\mathbf{p}-\mathbf{k}|)^{-r+2}(1+|\mathbf{k}|)^{s}+(1+|\mathbf{p}-\mathbf{k}|)^{-r+s+2}\right)\left|\widehat{u}_{\mathbf{k}}\right| .
$$

Using again Cauchy-Schwarz on each sum on the right hand side, and

$$
(1+|\mathbf{p}-\mathbf{k}|)^{-2(r-s-2)} \leq c(1+|\mathbf{p}-\mathbf{k}|)^{-\frac{2}{3}(r-s-2)}(1+|\mathbf{p}|)^{-\frac{2}{3}(r-s-2)},
$$

then for $0 \leq s<r-5$

$$
\sum_{\mathbf{p} \in \mathbb{Z}^{2} \backslash\{0\}}(1+|\mathbf{p}|)^{2 s+2}\left|(\widehat{\mathfrak{Z} u})_{\mathbf{p}}\right|^{2} \leq c \varepsilon^{2} \sum_{\mathbf{k} \in \mathbb{Z}^{2} \backslash\{0\}}(1+|\mathbf{k}|)^{2 s}\left|\widehat{u}_{\mathbf{k}}\right|^{2}
$$

which yields the required estimate $(8.28)$ for $\mathfrak{Z}$.

\section{F Proof of Lemma 17 and Proposition 5}

The symbol $L_{q}+V_{q}$ of $\mathfrak{L}_{q}+\mathfrak{V}_{q}$ may be written as

$$
-\nu\left(k_{1}+\rho k_{2}\right)^{2}+\left\{k_{2}^{2}+2 a_{q} k_{2}\left(k_{1}+\rho k_{2}\right)+b_{q}\left(k_{1}+\rho k_{2}\right)^{2}\right\}^{1 / 2}+V_{q}(\mathbf{k}),
$$

where $a_{q}, b_{q}, V_{q}$ satisfy Lemma 14 . The aim is now to find where are the "bad" $\rho$ ' $s$, for a given $\nu$, and this, uniformly with respect to iteration points (for $q \rightarrow \infty$ ). First, let us consider the solutions $\rho$ of the equation $L_{q}+V_{q}=\omega$ where $\omega$ is chosen later, tending to 0 as $k_{2} \rightarrow \infty$. We observe that this equation is equivalent to

$$
\left(\rho+\frac{k_{1}}{k_{2}}\right)^{2}=\frac{1}{\nu\left|k_{2}\right|}\left\{1+2 a_{q}\left(\rho+\frac{k_{1}}{k_{2}}\right)+b_{q}\left(\rho+\frac{k_{1}}{k_{2}}\right)^{2}\right\}^{1 / 2}+\frac{V_{q}-\omega}{\nu k_{2}^{2}} .
$$

For $\left|k_{2}\right|>N, N$ being large enough, and $\rho \in(0, N)$, it is clear that $\left|\rho+\frac{k_{1}}{k_{2}}\right|$ is $O\left(1 /\left|k_{2}\right|^{1 / 2}\right)$, and $\left|k_{1}\right|<2 N\left|k_{2}\right|$. Since $\rho>0$, we have $k_{1} / k_{2}<0$, and without loss of generality, we assume that $k_{2}>0, k_{1}<0$. Then we have two equations for $\rho$ :

$\rho_{ \pm}=-\frac{k_{1}}{k_{2}} \pm \frac{1}{\left(\nu k_{2}\right)^{1 / 2}}\left[\left\{1+2 a_{q}\left(\rho_{ \pm}+\frac{k_{1}}{k_{2}}\right)+b_{q}\left(\rho_{ \pm}+\frac{k_{1}}{k_{2}}\right)^{2}\right\}^{1 / 2}+\frac{\left(V_{q}-\omega\right)}{k_{2}}\right]^{1 / 2}$

which may be solved by using the implicit function theorem for $N$ large enough. We then obtain $\rho_{ \pm}$as a smooth function of $\left(\nu, a_{q}, b_{q}, V_{q}, \omega\right)$ defining

$$
\rho_{ \pm}=\widetilde{\Lambda}_{ \pm}\left(\nu, k_{1}, k_{2}, \omega, a_{q}, b_{q}, V_{q}\right) .
$$


Moreover the functions $\widetilde{\Lambda}_{ \pm}$have the following representations:

$$
\begin{aligned}
\widetilde{\Lambda}_{ \pm}= & -\frac{k_{1}}{k_{2}} \pm \frac{1}{\left(\nu k_{2}\right)^{1 / 2}}+\frac{a_{q}}{2 \nu k_{2}} \pm\left(\frac{\left(2 b_{q}-a_{q}^{2}\right)}{8 \nu^{3 / 2}}+\frac{\left(V_{q}-\omega\right)}{2 \nu^{1 / 2}}\right) \frac{1}{k_{2}^{3 / 2}}+ \\
& +\frac{1}{k_{2}^{2}} R\left(a_{q}, b_{q}, V_{q}, \omega, k_{2}\right),
\end{aligned}
$$

asymptexpr rho

where $R$ is bounded and smooth in $\omega$ and in $a_{q}, b_{q}$ and $V_{q}$ which are Lipschitz functions of $\rho_{ \pm}$as shown by Lemma 14. For fixed $q$ and $\omega$, the equations $\rho_{ \pm}=$ $\widetilde{\Lambda}_{ \pm}\left(\nu, k_{1}, k_{2}, \omega, a_{q}, b_{q}, V_{q}\right)$ may be solved in $\rho_{ \pm}$by using a contraction mapping argument, which defines the two functions $\rho_{ \pm}=\Lambda_{q \pm}\left(\nu, k_{1}, k_{2}, \omega\right)$, which are continuously differentiable with respect to $\omega$. This defines a denumerable number of curves in the $(\rho, \nu)$ plane, where we are only interested in $(\rho, \nu) \in \omega_{\epsilon_{0}}$. From (F.2) it is clear that

$$
\partial_{\omega} \Lambda_{q \pm}=\mp\left(1 / 2 \nu^{1 / 2}\right) k_{2}^{-3 / 2}+O\left(k_{2}^{-2}\right)
$$

which means that for $N$ large enough, $\Lambda_{q \pm}$ are monotonous functions of $\omega$, with a bounded derivative of order $O\left(k_{2}^{-3 / 2}\right)$. Now for a certain $\alpha>0$, we consider the family of curves such that

$$
|\omega| \leq d\left|k_{2}\right|^{-\alpha-1 / 2} \text {. }
$$

From the expansion (F.2), it results that for a fixed $\left(q, k_{1}, k_{2}\right)$ the curves $\rho_{ \pm}=$ $\Lambda_{q \pm}\left(\nu, k_{1}, k_{2}, \omega\right)$ in the $(\rho, \nu)$ plane, are contained in a curved narrow strip, centered on the curve obtained for $\omega=0$, of thickness $\delta \rho^{ \pm}$bounded uniformly for $(\rho, \nu) \in \omega_{\epsilon_{0}}$ by

$$
\left|\delta \rho_{ \pm}\right| \leq c d\left|k_{2}\right|^{-2-\alpha}
$$

where $c$ is a constant not depending on $\left(q, k_{1}, k_{2}\right)$ provided that $\left|k_{2}\right|>N$. Consider now the influence of the iterations (varying $q$ ). Thanks to Lemma 14 on successive iterates and (F.2), we obtain (notice that the main influence comes from $\frac{a_{q}}{2 \nu k_{2}}$ ) for a fixed $\left(k_{1}, k_{2}, \omega\right)$

$$
\left|\Lambda_{(q+1) \pm}-\Lambda_{q \pm}\right| \leq c 2^{-q}|\varepsilon|^{m} k_{2}^{-1}
$$

where $c$ is independent of $\left(q, k_{1}, k_{2}, \nu, \omega\right)$, provided that $\nu$ is near $\nu_{c}$ and $\left|k_{2}\right|>N$.

Now denote by $K_{k_{2}}=(1+\alpha) \ln k_{2} / \ln 2$, then for a fixed $\left(k_{1}, k_{2}\right)$ the total width of the curved strip containing the curves $\rho_{ \pm}=\Lambda_{q \pm}\left(\nu, k_{1}, k_{2}, \omega\right)$ for $q>K_{k_{2}}$ is bounded by

$$
\sum_{q>K_{k_{2}}} \frac{c}{2^{q}} \frac{|\varepsilon|^{m}}{k_{2}}=c \frac{|\varepsilon|^{m}}{k_{2} 2^{K_{k_{2}}}}=c \frac{|\varepsilon|^{m}}{k_{2}{ }^{2+\alpha}} .
$$

It results that for $q>K_{k_{2}}=q_{0}$ and for $\omega$ satisfying (F.3) and fixed $\left(k_{1}, k_{2}\right)$, all the curves $\rho_{ \pm}=\Lambda_{q \pm}\left(\nu, k_{1}, k_{2}, \omega\right)$ lie in a narrow curved region of the $(\rho, \nu)$ plane, of thickness $c k_{2}^{-2-\alpha} /\left(d+|\varepsilon|^{m}\right)$, and containing the curve $\rho_{ \pm}=\Lambda_{q_{0} \pm}\left(\nu, k_{1}, k_{2}, 0\right)$. For $q \leq K_{k_{2}}=q_{0}$ we may estimate the total thickness of the strips containing the strips associated with the rest of the curves, by

$$
c d K_{k_{2}}\left|k_{2}\right|^{-2-\alpha}=(1+\alpha) c d(\ln 2)^{-1} k_{2}^{-2-\alpha} \ln k_{2} .
$$

We obtain for fixed $\left(k_{1}, k_{2}\right)$, the total thickness of the "bad" strips centered on the curves $\rho_{ \pm}=\Lambda_{q_{0}}\left(\nu, k_{1}, k_{2}, 0\right)$ plus a finite number of strips centered on the curves $\rho_{ \pm}=\Lambda_{q \pm}\left(\nu, k_{1}, k_{2}, 0\right), q \leq q_{0}: \quad c^{\prime} \ln \left|k_{2}\right|\left|k_{2}\right|^{-2-\alpha}$. Notice that this thickness is independent of $\nu$ in the neighborhood of $\nu_{c}$. Now we consider the total set of bad strips for $\left(k_{1}, k_{2}\right) \in \mathbb{Z}^{2},\left|k_{2}\right|>N$, not forgetting that $\left|k_{1}\right|<2 N\left|k_{2}\right|$. In fact, since we consider the domain $\omega_{\epsilon_{0}}$ for $(\rho, \nu)$, we may observe, thanks to (F.2), that for $\left|\rho_{ \pm}-\lambda\right|+\left|\nu-\nu_{c}\right| \leq r$, the relevant values of $k_{2}$ giving eligible "bad" $\rho^{\prime} s$ in this square of the plane, are such that (from (F.4)) for $N$ large enough $k_{2}^{2+\alpha} \geq c / r$. Moreover, thanks to (F.2) we also have, for $N$ large enough

$$
\partial_{k_{1}}\left(k_{2} \Lambda_{q \pm}\right)=-1+O\left(|\varepsilon|^{m}\right),
$$


hence $\left|\Lambda_{q \pm}-\lambda\right| \leq r$ leads to ||$k_{1}\left|-g\left(\left|k_{2}\right|\right)\right| \leq c r\left|k_{2}\right|$, where $g\left(\left|k_{2}\right|\right)$ does not depend on $k_{1}$. It then results that the total width of the bad strips is bounded by

$$
\begin{aligned}
\sum_{|| k_{1}\left|-g\left(\left|k_{2}\right|\right)\right|<r\left|k_{2}\right|,\left|k_{2}\right|>c / r^{(2+\alpha)^{-1}}} \frac{c^{\prime} \ln \left|k_{2}\right|}{\left|k_{2}\right|^{2+\alpha}} & \leq \sum_{\left|k_{2}\right|>c / r^{(2+\alpha)}-1} \frac{2 r c c^{\prime} \ln \left|k_{2}\right|}{\left|k_{2}\right|^{1+\alpha}} \\
& \leq c^{\prime \prime} r^{\kappa}
\end{aligned}
$$

with $\kappa=1+\alpha^{\prime} /(2+\alpha), \alpha^{\prime}=\alpha / 2$. It then results that for $\nu$ fixed in a neighborhood of $\nu_{c}$, there is a set $\mathcal{E}_{N}^{\prime}(\nu)$ such that for $\rho \in \mathcal{E}_{N}^{\prime}(\nu)$ the following estimate holds true, uniformly in $q$ :

$$
\left|L_{q}\left(\rho, \nu, k_{1}, k_{2}\right)+V_{q}\left(\rho, \nu, k_{1}, k_{2}\right)\right| \geq d k_{2}^{-\alpha-1 / 2} .
$$

Moreover, taking into account of the condition (6.4), and taking an eligible $\nu \in$ $\left.\omega_{\epsilon}\right|_{\rho} \neq \varnothing$, the measure of $\left.\mathcal{E}_{N}^{\prime}(\nu) \cap \omega_{\epsilon}\right|_{\nu}$ is at least meas $\left(\left.\omega_{\epsilon}\right|_{\nu}\right)-c^{\prime \prime} \epsilon^{\kappa}$. Since the measure of $\left.\omega_{\epsilon}\right|_{\nu}$ is of order $\epsilon$ due to the diffeomorphism of Lemma 6 , this ends the proof of Proposition 5.

The proof of Lemma 17 follows the same lines, with the simplification due to the absence of iteration index $q, L_{c}\left(\lambda, \nu_{c}, \mathbf{k}\right)$ being given by (10.17). We then arrive (recall that here $\nu=\nu_{c}$ ) for $N$ large enough to a total width of the bad strips bounded by

$$
\sum_{\left|k_{1}\right|<2 N\left|k_{2}\right|,\left|k_{2}\right|>N} \frac{c_{N} d}{\left|k_{2}\right|^{2+\alpha}} \leq c d .
$$

Since $d$ is arbitrarily chosen, this proves Lemma 17 .

\section{G Computation of coefficients in Lemma 18}

The first step consists in identifying the operator $P_{0} \mathfrak{L}^{(2)} P_{0}-P_{0} \mathfrak{L}^{(1)} \mathfrak{L}_{c}^{-1} \mathfrak{L}^{(1)} P_{0}$ from the linear differential equation (7.29), before the normalization process. Let us formally define, as in (11.10)

$$
\begin{aligned}
& \frac{1}{H^{2} \widetilde{\mathfrak{a}}} \mathcal{D}^{2}+K \overline{\mathcal{G}}+p_{1} \mathcal{D}+\mathcal{H}_{-1}=\widetilde{\mathfrak{L}}_{c}+\widetilde{\mathfrak{L}}^{(1)}+\widetilde{\mathfrak{L}}^{(2)}+\text { h.o.t. } \\
\mathfrak{S} \circ \mathfrak{g} \mathbb{I}=\mathfrak{S}^{(0)}+\mathfrak{S}^{(1)}+\mathfrak{S}^{(2)}+\text { h.o.t. } & \mathfrak{T}=\mathbb{I}+\mathfrak{T}^{(1)}+\mathfrak{T}^{(2)}+\text { h.o.t. }
\end{aligned}
$$

where (see Theorem 8 and $(9.27)) \mathfrak{S}^{(0)}=\mathfrak{g}^{(0)} \mathbb{I}$ and $\mathfrak{g}^{(0)}=\lambda^{-1}\left(\tau_{1}+\tau_{2}\right)^{-1}$. Then from formula (9.19), we have

$$
\left.\mathfrak{S} \circ \mathfrak{g} \mathbb{I} \frac{1}{H^{2} \widetilde{\mathfrak{a}}} \mathcal{D}^{2}+K \overline{\mathcal{G}}+p_{1} \mathcal{D}+\mathcal{H}_{-1}\right\} \mathfrak{T}=\Pi(\mathfrak{L}+\mathfrak{V}+\mathfrak{F}) \Pi+(\mathbb{I}-\Pi)(\mathfrak{L}+\mathfrak{P}) .
$$

This leads to

$$
\begin{aligned}
& \mathfrak{L}_{c}=\mathfrak{g}^{(0)} \widetilde{\mathfrak{L}}_{c}, \quad \mathfrak{L}^{(1)}=\mathfrak{g}^{(0)} \widetilde{\mathfrak{L}}^{(1)}+\mathfrak{S}^{(1)} \widetilde{\mathfrak{L}}_{c}+\mathfrak{g}^{(0)} \widetilde{\mathfrak{L}}_{c} \mathfrak{T}^{(1)}, \\
& \mathfrak{L}^{(2)}=\mathfrak{g}^{(0)} \widetilde{\mathfrak{L}}^{(2)}+\mathfrak{S}^{(1)} \widetilde{\mathfrak{L}}^{(1)}+\mathfrak{g}^{(0)} \widetilde{\mathfrak{L}}^{(1)} \mathfrak{T}^{(1)}+\mathfrak{g}^{(0)} \widetilde{\mathfrak{L}}_{c} \mathfrak{T}^{(2)}+ \\
& +\mathfrak{S}^{(1)} \widetilde{\mathfrak{L}}_{c} \widetilde{\mathfrak{T}}^{(1)}+\mathfrak{S}^{(2)} \widetilde{\mathfrak{L}}_{c} .
\end{aligned}
$$

Then, by using the identities $P_{0} \widetilde{\mathfrak{L}}_{c}=\widetilde{\mathfrak{L}}_{c} P_{0}=0$, and the fact that $\mathfrak{g}^{(0)}$ is a constant, we obtain easily

$$
P_{0} \mathfrak{L}^{(2)} P_{0}-P_{0} \mathfrak{L}^{(1)} \mathfrak{L}_{c}^{-1} \mathfrak{L}^{(1)} P_{0}=\mathfrak{g}^{(0)}\left\{P_{0} \widetilde{\mathfrak{L}}^{(2)} P_{0}-P_{0} \widetilde{\mathfrak{L}}^{(1)} \widetilde{\mathfrak{L}}_{c}^{-1} \widetilde{\mathfrak{L}}^{(1)} P_{0}\right\}
$$

Next, we identify the principal part of operator $\mathcal{H}_{-1}$. We show the following 
Proposition 6 The linear operator $\mathcal{H}_{-1}$ is of order $|\varepsilon|^{2}$ and its principal part satisfies

$$
\mathcal{H}_{-1}^{(2)} u=P_{0} \mathcal{H}_{-1}^{(2)} P_{0} u .
$$

Moreover, by using the coordinates of $P_{0} u$ defined as $P_{0} u=\gamma_{1} \sin y_{1}+\gamma_{2} \sin y_{2}$, then the matrix of $P_{0} \mathcal{H}_{-1}^{(2)} P_{0}$ in the basis $\left\{\sin y_{1}, \sin y_{2}\right\}$ has the following form

$P_{0} \mathcal{H}_{-1}^{(2)} P_{0}=\frac{1}{\mu_{c}}\left(\begin{array}{cc}\varepsilon_{1}^{2}\left(16 \tau_{1}^{4}-4 \tau_{1}^{2}-17\right) & -\varepsilon_{1} \varepsilon_{2} \lambda^{2}\left(17+20 \tau_{2}^{2}+\frac{16 \lambda^{2}}{\mu_{c}^{2}} \tau_{1} \tau_{2}\right) \\ -\varepsilon_{1} \varepsilon_{2} \lambda^{2}\left(17+20 \tau_{1}^{2}+\frac{16}{\mu_{c}^{2}} \tau_{1} \tau_{2}\right) & \varepsilon_{2}^{2} \lambda^{4}\left(16 \tau_{2}^{4}-4 \tau_{2}^{2}-17\right)\end{array}\right)$

Remark 12 Notice that the above proposition allows to identify the contribution of $\mathcal{H}_{-1}$ to the linear operator in formula (G.1).

Proof Recall definitions (7.30) and (7.26) of operator $\mathcal{H}_{-1}$ and the functionals $\boldsymbol{\Xi}_{i}$ involved in (7.30). We may observe that the coefficients $p_{3}$ and $\mathbf{p}_{2}$ in (7.30) are of order $O(|\varepsilon|)$, and that the integral in the formulae for $\boldsymbol{\Xi}_{j}[u, \mathbf{0}]$ is also of order $O(|\varepsilon|)$, hence $\mathcal{H}_{-1}$ is $O\left(|\varepsilon|^{2}\right)$. Let us compute exactly this principal part. It is clear from $(7.23),(7.9)$ and from Theorem 4 , that

$$
\begin{gathered}
\widetilde{\psi}^{(1)}=-2 \varepsilon_{1} \sin y_{1}-2 \varepsilon_{2} \sin y_{2}, \quad \widetilde{\eta}^{(1)}=\frac{2 \varepsilon_{1}}{\mu_{c}} \cos y_{1}+\frac{2 \varepsilon_{2} \lambda}{\mu_{c}} \cos y_{2}, \\
\mathbf{p}_{2}^{(1)}=\frac{1}{\mu_{c}} \mathcal{D}_{c} \widetilde{\nabla_{X} \psi^{(1)}}-\nabla_{X} \eta^{(1)}, \quad p_{3}^{(1)}=\frac{1}{\mu_{c}} \mathcal{D}_{c} \widetilde{\eta}^{(1)},
\end{gathered}
$$

hence

$$
\left(\mathbf{p}_{2} \cdot \mathbf{u}^{\perp}\right)^{(1)}=\frac{4 \tau_{1}}{\mu_{c}} \varepsilon_{1} \sin y_{1}-\frac{4 \lambda^{2} \tau_{2}}{\mu_{c}} \varepsilon_{2} \sin y_{2}, \quad p_{3}^{(1)}=-\frac{2}{\mu_{c}^{2}} \varepsilon_{1} \sin y_{1}-\frac{2 \lambda^{2}}{\mu_{c}^{2}} \varepsilon_{2} \sin y_{2},
$$

and it is clear that the principal part of $\mathcal{H}_{-1}$ takes its values in ker $\mathfrak{L}_{c}$. Now, since the integral in formulae (7.26) for $\boldsymbol{\Xi}_{j}[u, \mathbf{0}]$ is of order $O(|\varepsilon|)$, we just need

$$
\left(\frac{-1}{\boldsymbol{\xi} \cdot \boldsymbol{\zeta}^{\perp}} \boldsymbol{\zeta}^{\perp}\right)^{(0)}=\frac{-3 \mu_{c}}{4 \pi^{2} \lambda\left(\tau_{1}+\tau_{2}\right)}\left(\lambda \tau_{2}, \tau_{1}\right) \quad\left(\frac{-1}{\boldsymbol{\zeta} \cdot \boldsymbol{\xi}^{\perp}} \boldsymbol{\xi}^{\perp}\right)^{(0)}=\frac{-1}{4 \pi^{2} \lambda\left(\tau_{1}+\tau_{2}\right)}(\lambda,-1),
$$

as noticed in Remark 7, and it is sufficient to compute the first order in $\left(\varepsilon_{1}, \varepsilon_{2}\right)$ of the integral

$$
\int_{\mathbb{R}^{2} /(2 \pi \mathbb{Z})^{2}} \mathbf{T}\left[\nabla u,-\frac{1}{H \mathfrak{a}} \mathcal{D} u\right] d Y,
$$

in (7.26) given by the integral of

$$
\begin{aligned}
& \varrho_{c}\left\{\frac{1}{3 \mu_{c}}\left(\mathbb{G}_{c}^{-1} \cdot \nabla \widetilde{\mathfrak{b}}^{(1)}\right) \cdot \nabla u+\left(\frac{1}{3 H(\widetilde{\mathbb{Q}} \widetilde{V} \cdot \widetilde{V})}-\frac{\widetilde{\mathfrak{a}}}{3 \mu_{c}}\right)^{(1)} \mathcal{D}_{c} u-\frac{1}{3 \mu_{c}}\left(\frac{1}{H \widetilde{\mathfrak{a}}}\right)^{(1)} \mathcal{D}_{c}^{3} u\right\}+ \\
& +\frac{1}{\mu_{c}}\left(\mathbb{G}_{c}^{-1} \cdot \nabla \widetilde{\mathfrak{b}}^{(1)}\right) \mathcal{D}_{c} u-\frac{1}{\mu_{c}}\left(\mathbb{G}_{c}^{-1} \cdot \nabla \widetilde{\eta}^{(1)}\right) \mathcal{D}_{c}^{2} u-\left(H \mathbb{G}^{-1}\right)^{(1)} \nabla u .
\end{aligned}
$$

integrand

From the computation of $\mathfrak{b}$ made in Appendix B, we have

$$
\widetilde{\mathfrak{b}}^{(1)}=-2 \mu_{c}^{-1} \varepsilon_{1} \sin y_{1}-2 \lambda^{2} \mu_{c}^{-1} \varepsilon_{2} \sin y_{2}=\mathcal{D}_{c} \widetilde{\eta}^{(1)},
$$


hence, after an integration by parts

$$
\begin{aligned}
& \int_{\mathbb{R}^{2} /(2 \pi \mathbb{Z})^{2}}\left\{\frac{1}{\mu_{c}}\left(\mathbb{G}_{c}^{-1} \cdot \nabla \widetilde{\mathfrak{b}}^{(1)}\right) \mathcal{D}_{c} u-\frac{1}{\mu_{c}}\left(\mathbb{G}_{c}^{-1} \cdot \nabla \widetilde{\eta}^{(1)}\right) \mathcal{D}_{c}^{2} u\right\} d Y \\
= & -\int_{\mathbb{R}^{2} /(2 \pi \mathbb{Z})^{2}}\left\{\frac{2}{\mu_{c}} \mathcal{D}_{c}\left(\mathbb{G}_{c}^{-1} \cdot \nabla \widetilde{\mathfrak{b}}^{(1)}\right)\right\} u d Y .
\end{aligned}
$$

Now, from the expression of $g_{i j}$ given in Appendix C, we have

$$
\mathbb{G}_{c}^{-1}=\left(\begin{array}{cc}
1+\tau_{1}^{2} & \lambda\left(1-\tau_{1} \tau_{2}\right) \\
\lambda\left(1-\tau_{1} \tau_{2}\right) & \lambda^{2}\left(1+\tau_{2}^{2}\right)
\end{array}\right),
$$

hence

$$
\frac{-2}{\mu_{c}} \mathcal{D}_{c}\left(\mathbb{G}_{c}^{-1} \cdot \nabla \widetilde{\mathfrak{b}}^{(1)}\right)=-\frac{4}{\mu_{c}^{2}}\left(\begin{array}{c}
\varepsilon_{1}\left(1+\tau_{1}^{2}\right) \sin y_{1}+\varepsilon_{2} \lambda^{4}\left(1-\tau_{1} \tau_{2}\right) \sin y_{2} \\
\varepsilon_{1} \lambda\left(1-\tau_{1} \tau_{2}\right) \sin y_{1}+\varepsilon_{2} \lambda^{5}\left(1+\tau_{2}^{2}\right) \sin y_{2}
\end{array}\right) .
$$

integrand1a

Now $\left(H \mathbb{G}^{-1}\right)^{(1)}$ comes from the expressions of $H$ and $g_{i j}$ given in Appendix C:

$$
\left(H \mathbb{G}^{-1}\right)^{(1)}=\varepsilon_{1} \cos y_{1}\left(\mathbb{G}_{c}^{-1}-\mathbb{G}_{c}^{-1} \mathbb{G}^{(1,0)} \mathbb{G}_{c}^{-1}\right)+\varepsilon_{2} \cos y_{2}\left(\lambda \mathbb{G}_{c}^{-1}-\mathbb{G}_{c}^{-1} \mathbb{G}^{(0,1)} \mathbb{G}_{c}^{-1}\right),
$$

with

It results that

$$
\begin{aligned}
\mathbb{G}^{(1,0)} & =\frac{1}{\lambda\left(\tau_{1}+\tau_{2}\right)}\left(\begin{array}{cc}
-2 \lambda\left(2 \tau_{1}+\tau_{2}\right) & \tau_{1} \\
\tau_{1} & 0
\end{array}\right), \\
\mathbb{G}^{(0,1)} & =\frac{1}{\lambda\left(\tau_{1}+\tau_{2}\right)}\left(\begin{array}{cc}
0 & \lambda \tau_{2} \\
\lambda \tau_{2} & -2\left(\tau_{1}+2 \tau_{2}\right)
\end{array}\right) .
\end{aligned}
$$

$$
\left(H \mathbb{G}^{-1}\right)^{(1)}=\varepsilon_{1} \cos y_{1}\left(H \mathbb{G}^{-1}\right)^{(1,0)}+\varepsilon_{2} \cos y_{2}\left(H \mathbb{G}^{-1}\right)^{(0,1)},
$$

with

$$
\begin{aligned}
\left(H \mathbb{G}^{-1}\right)^{(1,0)} & =\left(\begin{array}{cc}
\left(1+\tau_{1}^{2}\right)\left(3+4 \tau_{1}^{2}\right) & \lambda\left(1+\tau_{1}^{2}\right)\left(3-4 \tau_{1} \tau_{2}\right) \\
\lambda\left(1+\tau_{1}^{2}\right)\left(3-4 \tau_{1} \tau_{2}\right) & \lambda^{2}\left[\tau_{2}^{2}\left(1+\tau_{1}^{2}\right)+3\left(1-\tau_{1} \tau_{2}\right)^{2}\right]
\end{array}\right) \\
\left(H \mathbb{G}^{-1}\right)^{(0,1)} & =\left(\begin{array}{cc}
\lambda\left[\tau_{1}^{2}\left(1+\tau_{2}^{2}\right)+3\left(1-\tau_{1} \tau_{2}\right)^{2}\right] & \lambda^{2}\left(1+\tau_{2}^{2}\right)\left(3-4 \tau_{1} \tau_{2}\right) \\
\lambda^{2}\left(1+\tau_{2}^{2}\right)\left(3-4 \tau_{1} \tau_{2}\right) & \lambda^{3}\left(1+\tau_{2}^{2}\right)\left(3+4 \tau_{2}^{2}\right)
\end{array}\right) .
\end{aligned}
$$

Finally, after an integration by parts, we obtain

$$
\begin{aligned}
\int_{\mathbb{R}^{2} /(2 \pi \mathbb{Z})^{2}}-\left(H \mathbb{G}^{-1}\right)^{(1)} \nabla u d Y= & -\frac{2 \pi^{2} \varepsilon_{1} \gamma_{1}}{\mu_{c}^{2}}\left(\begin{array}{c}
3+4 \tau_{1}^{2} \\
\lambda\left(3-4 \tau_{1} \tau_{2}\right)
\end{array}\right)+ \\
& -\frac{2 \pi^{2} \varepsilon_{2} \gamma_{2} \lambda^{4}}{\mu_{c}^{2}}\left(\begin{array}{c}
\left(3-4 \tau_{1} \tau_{2}\right) \\
\lambda\left(3+4 \tau_{2}^{2}\right)
\end{array}\right) .
\end{aligned}
$$

Then we obtain

$$
\begin{gathered}
\int_{\mathbb{R}^{2} /(2 \pi \mathbb{Z})^{2}}\left\{\frac{1}{\mu_{c}}\left(\mathbb{G}_{c}^{-1} \cdot \nabla \widetilde{\mathfrak{b}}^{(1)}\right) \mathcal{D}_{c} u-\frac{1}{\mu_{c}}\left(\mathbb{G}_{c}^{-1} \cdot \nabla \widetilde{\eta}^{(1)}\right) \mathcal{D}_{c}^{2} u-\left(H \mathbb{G}^{-1}\right)^{(1)} \nabla u\right\} d Y= \\
=-\frac{2 \pi^{2}}{\mu_{c}^{2}} \varepsilon_{1} \gamma_{1}\left(\begin{array}{c}
7+8 \tau_{1}^{2} \\
\lambda\left(7-8 \tau_{1} \tau_{2}\right)
\end{array}\right)-\frac{2 \pi^{2} \lambda^{4}}{\mu_{c}^{2}} \varepsilon_{2} \gamma_{2}\left(\begin{array}{c}
7-8 \tau_{1} \tau_{2} \\
\lambda\left(7+8 \tau_{2}^{2}\right)
\end{array}\right) .
\end{gathered}
$$


Since the other part of the integrand is colinear to $\varrho_{c}=(1, \lambda)$, we already obtain

$$
\left(\boldsymbol{\Xi}_{2}[u, \mathbf{0}]\right)^{(1)}=\frac{4}{\mu_{c}^{2}}\left(\tau_{1} \varepsilon_{1} \gamma_{1}-\lambda^{4} \tau_{2} \varepsilon_{2} \gamma_{2}\right) .
$$

The contribution $\left(\boldsymbol{\Xi}_{11}[u, \mathbf{0}]\right)^{(1)}$ to $\left(\boldsymbol{\Xi}_{1}[u, \mathbf{0}]\right)^{(1)}$ of the part of the integrand non colinear to $\varrho_{c}$, is then given by

$$
\left(\boldsymbol{\Xi}_{11}[u, \mathbf{0}]\right)^{(1)}=\frac{21}{2 \mu_{c}}\left(\varepsilon_{1} \gamma_{1}+\lambda^{4} \varepsilon_{2} \gamma_{2}\right) .
$$

It remains to compute the factor of $\varrho_{c}$ in the integrand. From Appendix B we have $\widetilde{\mathfrak{a}}^{(1)}$, and from Appendix $\mathrm{C}$ for $H^{(1)}$ and $(\widetilde{\mathbb{Q}} \widetilde{V} \cdot \widetilde{V})^{(1)}$ we then obtain

$$
\begin{gathered}
\left(\frac{1}{3 H(\widetilde{\mathbb{Q}} \widetilde{V} \cdot \widetilde{V})}\right)^{(1)}-\frac{1}{3 \mu_{c}} \mathcal{D}_{c}^{2}\left(\frac{1}{H \widetilde{\mathfrak{a}}}\right)^{(1)}-\frac{(\widetilde{\mathfrak{a}})^{(1)}}{3 \mu_{c}}=\left(\frac{3+2 \tau_{1}^{2}}{3 \mu_{c}^{2}}+1\right) \varepsilon_{1} \cos y_{1}+ \\
+\lambda\left(\lambda^{2} \frac{3+2 \tau_{2}^{2}}{3 \mu_{c}^{2}}+1\right) \varepsilon_{2} \cos y_{2}, \\
\frac{1}{3 \mu_{c}}\left(\mathbb{G}_{c}^{-1} \cdot \nabla \widetilde{\mathfrak{b}}^{(1)}\right)=-\frac{2}{3 \mu_{c}^{2}}\left(\begin{array}{c}
\left(1+\tau_{1}^{2}\right) \varepsilon_{1} \cos y_{1}+\lambda^{3}\left(1-\tau_{1} \tau_{2}\right) \varepsilon_{2} \cos y_{2} \\
\lambda\left(1-\tau_{1} \tau_{2}\right) \varepsilon_{1} \cos y_{1}+\lambda^{4}\left(1+\tau_{2}^{2}\right) \varepsilon_{2} \cos y_{2}
\end{array}\right),
\end{gathered}
$$

which leads to the contribution $\left(\boldsymbol{\Xi}_{12}[u, \mathbf{0}]\right)^{(1)}$ to $\left(\boldsymbol{\Xi}_{1}[u, \mathbf{0}]\right)^{(1)}$ of the part of the integrand colinear to $\varrho_{c}$

$$
\left(\boldsymbol{\Xi}_{12}[u, \mathbf{0}]\right)^{(1)}=-\frac{\mu_{c}}{2}\left[\left(4+\tau_{1}^{2}\right) \varepsilon_{1} \gamma_{1}+\lambda^{2}\left(4+\tau_{2}^{2}\right) \varepsilon_{2} \gamma_{2}\right],
$$

and finally

$$
\left(\boldsymbol{\Xi}_{1}[u, \mathbf{0}]\right)^{(1)}=\frac{\mu_{c}}{2}\left[\left(17+20 \tau_{1}^{2}\right) \varepsilon_{1} \gamma_{1}+\lambda^{2}\left(17+20 \tau_{2}^{2}\right) \varepsilon_{2} \gamma_{2}\right] .
$$

Proposition 6 is then proved.

It remains to study the contribution of the linear operator $\frac{1}{H^{2} \widetilde{a}} \mathcal{D}^{2}+K \overline{\mathcal{G}}+p_{1} \mathcal{D}$ to formula (G.1), noticing that by construction $K=1+O\left(|\varepsilon|^{2 m+1}\right)$. The idea is to make explicit the connection between the formulation as a second order scalar operator and the formulation as a first order 2-components linear operator, coming from the linearization of $\mathcal{F}(U, \mu, \mathbf{u})$. Let us define the expansion of the approximate solution $U_{2 m}$ with $A=\varepsilon_{1}, B=\varepsilon_{2}$ real positive as found in Theorem 4

$$
U_{2 m}=\varepsilon_{1} U^{(1,0)}+\varepsilon_{2} U^{(0,1)}+\varepsilon_{1}^{2} U^{(2,0)}+\varepsilon_{1} \varepsilon_{2} U^{(1,1)}+\varepsilon_{2}^{2} U^{(0,2)}+\text { h.o.t. }
$$

where

$$
U^{(1,0)}=\left(\begin{array}{c}
-2 \sin K_{1} \cdot X \\
\frac{2}{\mu_{c}} \cos K_{1} \cdot X
\end{array}\right), U^{(0,1)}=\left(\begin{array}{c}
-2 \sin K_{2} \cdot X \\
\frac{2 \lambda}{\mu_{c}} \cos K_{2} \cdot X
\end{array}\right) .
$$

The formal computation using Lyapunov-Schmidt method which was made in Appendix A, for finding the bifurcation equations (A.9), may be rewritten here as

$$
\begin{aligned}
\frac{2|\Omega|}{\mu_{c}} \varepsilon_{1}\left(\frac{\widetilde{\mu}}{\mu_{c}}-2\left(K_{1} \cdot \boldsymbol{\omega}\right)+a_{1} \varepsilon_{1}^{2}+b_{1} \varepsilon_{2}^{2}+\text { h.o.t. }\right) & =0 \\
\frac{2|\Omega| \lambda^{2}}{\mu_{c}} \varepsilon_{1}\left(\frac{\widetilde{\mu}}{\mu_{c}}-\frac{2}{\lambda}\left(K_{2} \cdot \boldsymbol{\omega}\right)+a_{2} \varepsilon_{1}^{2}+b_{2} \varepsilon_{2}^{2}+\text { h.o.t. }\right) & =0,
\end{aligned}
$$


where $a_{j}, b_{j}$ are the coefficients given explicitly in Appendix A. We now expand the differential in powers of $\left(\varepsilon_{1}, \varepsilon_{2}\right)$

$$
\mathcal{L}=\partial_{U} \mathcal{F}\left(U_{m}, \mu, \mathbf{u}\right)=\mathcal{L}^{(0)}+\mathcal{L}^{(1)}+\mathcal{L}^{(2)}+\text { h.o.t. }
$$

where the operator $\mathcal{L}^{(0)}$ is formally invertible in the subspace $\left(\operatorname{ker} \mathcal{L}^{(0)}\right)^{\perp}$. Then we have the following

Proposition 7 The following identities hold for any $\left(\gamma_{1}, \gamma_{2}\right) \in \mathbb{R}^{2}$ :

$$
\begin{gathered}
\left\langle\mathcal{L}^{(2)}\left(\gamma_{1} U^{(1,0)}+\gamma_{2} U^{(0,1)}\right)-\mathcal{L}^{(1)} \mathcal{L}^{(0)-1} \mathcal{L}^{(1)}\left(\gamma_{1} U^{(1,0)}+\gamma_{2} U^{(0,1)}\right), U^{(1,0)}\right\rangle= \\
=\frac{4|\Omega|}{\mu_{c}}\left(a_{1} \varepsilon_{1}^{2} \gamma_{1}+b_{1} \varepsilon_{1} \varepsilon_{2} \gamma_{2}\right), \\
\left\langle\mathcal{L}^{(2)}\left(\gamma_{1} U^{(1,0)}+\gamma_{2} U^{(0,1)}\right)-\mathcal{L}^{(1)} \mathcal{L}^{(0)-1} \mathcal{L}^{(1)}\left(\gamma_{1} U^{(1,0)}+\gamma_{2} U^{(0,1)}\right), U^{(0,1)}\right\rangle= \\
=\frac{4|\Omega| \lambda^{2}}{\mu_{c}}\left(a_{2} \varepsilon_{1} \varepsilon_{2} \gamma_{1}+b_{2} \varepsilon_{2}^{2} \gamma_{2}\right) .
\end{gathered}
$$

Proof In an identical way as shown in Lemma 18, the formal computation of the inverse of operator $\mathcal{L}$, leads to a linear two-dimensional system in the Kernel of $\mathcal{L}^{(0)}$, of the form

$$
\mathcal{P}_{0} \mathcal{L}^{(2)} \mathcal{P}_{0}-\mathcal{P}_{0} \mathcal{L}^{(1)} \mathcal{L}^{(0)-1} \mathcal{L}^{(1)} \mathcal{P}_{0}+\text { h.o.t. }
$$

where $\mathcal{P}_{0}$ is the projection defined in Appendix A, which may be as well obtained in differentiating the system of bifurcation equations (G.11) with respect to $\left(\varepsilon_{1}, \varepsilon_{2}\right)$. This leads to a linear operator mapping $\gamma_{1} U^{(1,0)}+\gamma_{2} U^{(0,1)}$ into the following two components:

$$
\begin{aligned}
& \frac{2|\Omega|}{\mu_{c}}\left(\left[\frac{\tilde{\mu}}{\mu_{c}}-2\left(K_{1} \cdot \boldsymbol{\omega}\right)+3 a_{1} \varepsilon_{1}^{2}+b_{1} \varepsilon_{2}^{2}\right] \gamma_{1}+2 b_{1} \varepsilon_{1} \varepsilon_{2} \gamma_{2}\right)+\text { h.o.t. } \\
& \frac{2|\Omega| \lambda^{2}}{\mu_{c}}\left(2 a_{2} \varepsilon_{1} \varepsilon_{2} \gamma_{1}+\left[\frac{\tilde{\mu}}{\mu_{c}}-\frac{2}{\lambda}\left(K_{2} \cdot \boldsymbol{\omega}\right)+a_{2} \varepsilon_{1}^{2}+3 b_{2} \varepsilon_{2}^{2}\right] \gamma_{2}\right)+\text { h.o.t. }
\end{aligned}
$$

and by using (G.11), this system becomes

$$
\left(\begin{array}{c}
\frac{4|\Omega|}{\mu_{c}}\left(a_{1} \varepsilon_{1}^{2} \gamma_{1}+b_{1} \varepsilon_{1} \varepsilon_{2} \gamma_{2}\right) \\
\frac{4|\Omega| \lambda^{2}}{\mu_{c}}\left(a_{2} \varepsilon_{1} \varepsilon_{2} \gamma_{1}+b_{2} \varepsilon_{2}^{2} \gamma_{2}\right)
\end{array}\right)+\text { h.o.t. }
$$

which proves the Proposition.

Our aim is now to use the above proposition to compute $P_{0} \widetilde{\mathfrak{L}}_{1}^{(2)} P_{0}-P_{0} \widetilde{\mathfrak{L}}^{(1)} \widetilde{\mathfrak{L}}_{c}^{-1} \widetilde{\mathfrak{L}}^{(1)} P_{0}$ where $\widetilde{\mathfrak{L}}_{1}^{(2)}=\widetilde{\mathfrak{L}}^{(2)}-\mathcal{H}_{-1}^{(2)}$. The first thing to do now is to write the two-components operator $\mathcal{L}$ in coordinate $Y$, and express the scalar products with integrals over a period in $Y$. We notice that

$$
\int_{\Omega} u(X) v(X) d X=\int_{T^{2}} \widetilde{u}(Y) \widetilde{v}(Y)\left|\operatorname{det} X^{\prime}(Y)\right| d Y,
$$

with

$$
\left|\operatorname{det} X^{\prime}(Y)\right|=\left|\operatorname{det} \mathbb{T}^{-1}\right|+O(|\varepsilon|),\left|\operatorname{det} \mathbb{T}^{-1}\right|=\frac{1}{\lambda\left(\tau_{1}+\tau_{2}\right)}=\frac{|\Omega|}{4 \pi^{2}}
$$


It results that with scalar products in coordinate $\mathrm{Y}$, we have

$$
\begin{gathered}
\left\langle\mathcal{L}^{(2)}\left(\gamma_{1} \widetilde{U}^{(1,0)}+\gamma_{2} \widetilde{U}^{(0,1)}\right)-\mathcal{L}^{(1)} \mathcal{L}^{(0)-1} \mathcal{L}^{(1)}\left(\gamma_{1} \widetilde{U}^{(1,0)}+\gamma_{2} \widetilde{U}^{(0,1)}\right), \widetilde{U}^{(1,0)}\right\rangle= \\
=\frac{4 \pi^{2}}{\mu_{c}}\left(a_{1} \varepsilon_{1}^{2} \gamma_{1}+b_{1} \varepsilon_{1} \varepsilon_{2} \gamma_{2}\right) \\
\left\langle\mathcal{L}^{(2)}\left(\gamma_{1} \widetilde{U}^{(1,0)}+\gamma_{2} \widetilde{U}^{(0,1)}\right)-\mathcal{L}^{(1)} \mathcal{L}^{(0)-1} \mathcal{L}^{(1)}\left(\gamma_{1} \widetilde{U}^{(1,0)}+\gamma_{2} \widetilde{U}^{(0,1)}\right), \widetilde{U}^{(0,1)}\right\rangle= \\
=\frac{4 \pi^{2} \lambda^{2}}{\mu_{c}}\left(a_{2} \varepsilon_{1} \varepsilon_{2} \gamma_{1}+b_{2} \varepsilon_{2}^{2} \gamma_{2}\right)
\end{gathered}
$$

with (renormalizing the eigenvectors)

$$
\widetilde{U}^{(1,0)}=\left(\begin{array}{c}
\sin y_{1} \\
\frac{-1}{\mu_{c}} \cos y_{1}
\end{array}\right), \widetilde{U}^{(0,1)}=\left(\begin{array}{c}
\sin y_{2} \\
\frac{-\lambda}{\mu_{c}} \cos y_{2}
\end{array}\right) .
$$

We now need to define precisely the linear operators occuring in the former expansions:

$$
\begin{aligned}
& \mathcal{L}=\left(\begin{array}{cc}
\overline{\mathcal{G}} & -\frac{1}{H} \mathcal{D}-q_{1} \\
\frac{1}{H} \mathcal{D} & \widetilde{\mathfrak{a}}
\end{array}\right), \quad \mathcal{L}^{(0)}=\left(\begin{array}{cc}
\overline{\mathcal{G}}^{(0)} & -\mathcal{D}_{c} \\
\mathcal{D}_{c} & \mu_{c}
\end{array}\right) \\
& \mathcal{L}^{(1)}=\left(\begin{array}{cc}
\overline{\mathcal{G}}^{(1)} & -\left(\frac{1}{H}\right)^{(1)} \mathcal{D}_{c}-q_{1}{ }^{(1)} \\
\left(\frac{1}{H}\right)^{(1)} \mathcal{D}_{c} & \widetilde{\mathfrak{a}}^{(1)}
\end{array}\right), \\
& \mathcal{L}^{(2)}=\left(\begin{array}{cc}
\overline{\mathcal{G}}^{(2)} & -\rho^{(2)} \partial_{y_{2}}-\left(\frac{1}{H}\right)^{(2)} \mathcal{D}_{c}-q_{1}^{(2)} \\
\rho^{(2)} \partial_{y_{2}}+\left(\frac{1}{H}\right)^{(2)} \mathcal{D}_{c} & \widetilde{\mathfrak{a}}^{(2)}
\end{array}\right), \\
& \widetilde{\mathfrak{L}}_{c}=\frac{1}{\mu_{c}} \mathcal{D}_{c}^{2}+\overline{\mathcal{G}}^{(0)}, \widetilde{\mathfrak{L}}^{(1)}=\left(\frac{1}{\widetilde{\mathfrak{a}} H^{2}}\right)^{(1)} \mathcal{D}_{c}^{2}+\overline{\mathcal{G}}^{(1)}+p_{1}^{(1)} \mathcal{D}_{c}, \\
& \widetilde{\mathfrak{L}}^{(2)}=\left(\frac{1}{\widetilde{\mathfrak{a}} H^{2}}\right)^{(2)} \mathcal{D}_{c}^{2}+\overline{\mathcal{G}}^{(2)}+p_{1}^{(2)} \mathcal{D}_{c}+\frac{2 \rho^{(2)}}{\mu_{c}} \partial_{y_{2}} \mathcal{D}_{c}, \\
& \text { with } p_{1}^{(1)}=\frac{q_{1}^{(1)}}{\mu_{c}}+\mathcal{D}_{c}\left(\frac{1}{\widetilde{\mathfrak{a}} H}\right)^{(1)}, \\
& p_{1}^{(2)}=\frac{q_{1}^{(2)}}{\mu_{c}}+\left(\frac{1}{\widetilde{\mathfrak{a}} H}\right)^{(1)} q_{1}^{(1)}+\mathcal{D}_{c}\left(\frac{1}{\widetilde{\mathfrak{a}} H}\right)^{(2)}+\left(\frac{1}{H}\right)^{(1)} \mathcal{D}_{c}\left(\frac{1}{\widetilde{\mathfrak{a}} H}\right)^{(1)} \text {. }
\end{aligned}
$$

Then, for $(u, \varsigma) \in\left(\operatorname{ker} \mathcal{L}^{(0)}\right)^{\perp}$ which is equivalent to $\left(u+\frac{1}{\mu_{c}} \mathcal{D}_{c} \varsigma\right) \in\left(\operatorname{ker} \widetilde{\mathfrak{L}}_{c}\right)^{\perp}=$ $\left(\text { ker } \mathfrak{L}_{c}\right)^{\perp}$, we have

$$
\mathcal{L}^{(0)-1}\left(\begin{array}{c}
u \\
\varsigma
\end{array}\right)=\left(\begin{array}{c}
\widetilde{\mathfrak{L}}_{c}^{-1}\left(u+\frac{1}{\mu_{c}} \mathcal{D}_{c} \varsigma\right) \\
-\frac{1}{\mu_{c}} \mathcal{D}_{c} \widetilde{\mathfrak{L}}_{c}^{-1}\left(u+\frac{1}{\mu_{c}} \mathcal{D}_{c} \varsigma\right)+\frac{1}{\mu_{c}} \varsigma
\end{array}\right) .
$$

Let us now denote by $\widetilde{U}^{(1)}$ one of the two eigenvectors $\widetilde{U}^{(1,0)}, \widetilde{U}^{(0,1)}$ and $u^{(1)}$ one of the two eigenvectors $\sin y_{1}$, sin $y_{2}$, such that $\widetilde{U}^{(1)}=\left(u^{(1)},-\frac{1}{\mu_{c}} \mathcal{D}_{c} u^{(1)}\right)^{t}$, and since $\mathcal{L}^{(1)} \widetilde{U}^{(1)} \in\left(\operatorname{ker} \mathcal{L}^{(0)}\right)^{\perp}$, it is easy to check that

$$
\mathcal{L}^{(0)-1} \mathcal{L}^{(1)} \widetilde{U}^{(1)}=\left(\begin{array}{c}
\widetilde{\mathfrak{L}}_{c}^{-1} \widetilde{\mathfrak{L}}^{(1)} u^{(1)} \\
-\frac{1}{\mu_{c}} \mathcal{D}_{c} \widetilde{\mathfrak{L}}_{c}^{-1} \widetilde{\mathfrak{L}}^{(1)} u^{(1)}+\left(\frac{1}{\tilde{\mathfrak{a}} H}\right)^{(1)} \mathcal{D}_{c} u^{(1)}
\end{array}\right),
$$


Now, for any smooth function $v$ and $\widetilde{V}^{(1)}=\left(v^{(1)},-\frac{1}{\mu_{c}} \mathcal{D}_{c} v^{(1)}\right) \in \operatorname{ker} \mathcal{L}^{(0)}$, we have

$$
\left\langle\mathcal{L}^{(1)}\left(\begin{array}{c}
v \\
-\frac{1}{\mu_{c}} \mathcal{D}_{c} v
\end{array}\right), \widetilde{V}^{(1)}\right\rangle=\left\langle\widetilde{\mathfrak{L}}^{(1)} v, v^{(1)}\right\rangle,
$$

from which it results that

$$
\begin{gathered}
\left\langle\mathcal{L}^{(1)} \mathcal{L}^{(0)-1} \mathcal{L}^{(1)} \widetilde{U}^{(1)}, \widetilde{V}^{(1)}\right\rangle=-\left\langle\mu_{c}\left(\frac{1}{\tilde{\mathfrak{a}} H}\right)^{(1)} \mathcal{D}_{c}\left[\left(\frac{1}{\tilde{\mathfrak{a}} H}\right)^{(1)} \mathcal{D}_{c} u^{(1)}\right], v^{(1)}\right\rangle+(\mathrm{G} .16) \text { decompformula1 } \\
+\left\langle\widetilde{\mathfrak{L}}^{(1)} \widetilde{\mathfrak{L}}_{c}^{-1} \widetilde{\mathfrak{L}}^{(1)} u^{(1)}, v^{(1)}\right\rangle+\left\langle\left(\mathcal{D}_{c}\left(\frac{\widetilde{\mathfrak{a}}^{(1)}}{\mu_{c}}\right)-q_{1}^{(1)}\right)\left(\frac{1}{\widetilde{\mathfrak{a}} H}\right)^{(1)} \mathcal{D}_{c} u^{(1)}, v^{(1)}\right\rangle .
\end{gathered}
$$

Now with $\mathcal{L}^{(2)}$ we have

$$
\begin{aligned}
\left\langle\mathcal{L}^{(2)} \widetilde{U}^{(1)}, \widetilde{V}^{(1)}\right\rangle= & \left\langle\widetilde{\mathfrak{L}}_{1}^{(2)} u^{(1)}, v^{(1)}\right\rangle-\left\langle\mu_{c}\left(\left(\frac{1}{\tilde{\mathfrak{a}} H}\right)^{(1)}\right)^{2} \mathcal{D}_{c}^{2} u^{(1)}, v^{(1)}\right\rangle+ \\
& -\left\langle\mu_{c}\left(\frac{1}{\tilde{\mathfrak{a}} H}\right)^{(1)} \mathcal{D}_{c}\left(\frac{1}{\widetilde{\mathfrak{a}} H}\right)^{(1)} \mathcal{D}_{c} u^{(1)}, v^{(1)}\right\rangle+ \\
& +\left\langle\left(\mathcal{D}_{c}\left(\frac{\widetilde{\mathfrak{a}}^{(1)}}{\mu_{c}}\right)-q_{1}^{(1)}\right)\left(\frac{1}{\widetilde{\mathfrak{a}} H}\right)^{(1)} \mathcal{D}_{c} u^{(1)}, v^{(1)}\right\rangle,
\end{aligned}
$$

hence finally from (G.16) and (G.17), and for any $\widetilde{U}^{(1)}, \widetilde{V}^{(1)} \in \operatorname{ker} \mathcal{L}^{(0)}$ we have the identity

$$
\begin{aligned}
& \left\langle\mathcal{L}^{(2)} \widetilde{U}^{(1)}, \widetilde{V}^{(1)}\right\rangle-\left\langle\mathcal{L}^{(1)} \mathcal{L}^{(0)-1} \mathcal{L}^{(1)} \widetilde{U}^{(1)}, \widetilde{V}^{(1)}\right\rangle \\
= & \left\langle\widetilde{\mathfrak{L}}_{1}^{(2)} u^{(1)}, v^{(1)}\right\rangle-\left\langle\widetilde{\mathfrak{L}}^{(1)} \widetilde{\mathfrak{L}}_{c}^{-1} \widetilde{\mathfrak{L}}^{(1)} u^{(1)}, v^{(1)}\right\rangle .
\end{aligned}
$$

Taking into account of the form of coefficients $a_{j}, b_{j}$ found in Appendix A, it results that in the basis $\left\{\sin y_{1}, \sin y_{2}\right\}$ the linear operator $P_{0} \widetilde{\mathfrak{L}}_{1}^{(2)} P_{0}-P_{0} \widetilde{\mathfrak{L}}^{(1)} \widetilde{\mathfrak{L}}_{c}^{-1} \widetilde{\mathfrak{L}}^{(1)} P_{0}$ has the following symmetric matrix

$$
\frac{2}{\mu_{c}}\left(\begin{array}{cc}
\frac{4}{\mu_{c}^{4}} \varepsilon_{1}^{2} & \lambda^{2} a_{2} \varepsilon_{1} \varepsilon_{2} \\
\lambda^{2} a_{2} \varepsilon_{1} \varepsilon_{2} & \frac{4 \lambda^{8}}{\mu_{c}^{4}} \varepsilon_{2}^{2}
\end{array}\right),
$$

where $a_{2}$ has a complicated expression given in Appendix A. After adding $P_{0} \mathcal{H}_{-1}^{(2)} P_{0}$ given in Proposition 6, we then have explicitly found the form of the operator $P_{0} \mathfrak{L}^{(2)} P_{0}-P_{0} \mathfrak{L}^{(1)} \mathfrak{L}_{c}^{-1} \mathfrak{L}^{(1)} P_{0}$ occuring in Lemma 18, with

$$
\begin{aligned}
& \widetilde{a}_{1}=3 \nu_{c}\left\{8 \tau_{1}^{4}+4 \tau_{1}^{2}-3\right\}, \widetilde{b}_{2}=3 \lambda^{4} \nu_{c}\left\{8 \tau_{2}^{4}+4 \tau_{2}^{2}-3\right\} \\
& \widetilde{b}_{1}=\lambda^{2} \nu_{c}\left\{2 a_{2}-\left(17+20 \tau_{2}^{2}+16\left(1+\tau_{2}^{2}\right) \tau_{1} \tau_{2}\right)\right\}, \\
& \widetilde{a}_{2}=\lambda^{2} \nu_{c}\left\{2 a_{2}-\left(17+20 \tau_{1}^{2}+16\left(1+\tau_{1}^{2}\right) \tau_{1} \tau_{2}\right)\right\} .
\end{aligned}
$$

atilde j, btilde $_{-} j$

This ends the proof of Lemma 18.

\section{References}

B-D-M 1. Bridges, T., Dias, F., Menasce, D.: Steady three-dimensional water-wave patterns on a finite-depth fluid. J.Fluid Mech. 436, 145-175 (2001)

2. Craig, W., Nicholls, D.:Traveling gravity water waves in two and three dimensions. EJMB/Fluids 21, 615-641 (2002) 
3. Craig, W., Nicholls D.: Travelling two and three-dimensional capillary gravity water waves. SIAM J. Math. Anal. 32, 323-359 (2000)

4. Craig, W., Schanz,U., Sulem, C.: The modulational regime of three-dimensional water waves and the Davey-Stewartson system. Ann. Inst. Henri Poincaré 14, 615-667 (1997)

5. Dias, F., Iooss, G.: Water waves as a spatial dynamical system. In Friedlander, S., Serre, D. (ed.) Handbook of Mathematical Fluid Dynamics, chap 10, 443 -499. Elsevier (2003)

6. Dias, F., Kharif, C.: Nonlinear gravity and capillary-gravity waves. Annu. Rev. Fluid Mech. 31, 301-346 (1999)

7. Fuchs, R.: On the theory of short-crested oscillatory waves. U.S. Natl. Bur. Stand. Circ. 521, 187-200 (1952)

8. Buffoni, B., Groves, M., Sun, S.M.: Existence and conditional energetic stability of three-dimensional fully localised solitary gravity-capillary water waves. Preprint (2009)

9. Groves, M.: An existence theory for three-dimensional periodic travelling gravity-capillary water waves with bounded transverse profiles. Physica D 152153, 395-415 (2001)

10. Groves,M., Haragus, M.: A bifurcation theory for three-dimensional oblique travelling gravity-capillary water waves. J.Nonlinear Sci. 13, 397-447 (2003)

11. Groves, M., Mielke, A.: A spatial dynamics approach to three-dimensional gravity-capillary steady water waves. Proc. Roy. Soc. Edin. A 131, 83-136 (2001)

12. Hammack, J., Henderson, D., Segur, H.: Progressive waves with persistent, twodimensional surface patterns in deep water. J.Fluid Mech. 532, 1-52 (2005)

13. Haragus-Courcelle, M., Kirchgässner, K.: Three-dimensional steady capillarygravity waves. In Fiedler, B. (ed.)Ergodic theory, Analysis and efficient simulation of dynamical systems, Berlin, 363-397. Springer-Verlag (2001)

14. Hu, B., Nicholls, D.: Analyticity of Dirichlet-Neumann operators on Hölder and Lipschitz domains. SIAM J. Math. Anal. 37, 302-320 (2005)

15. Iooss, G., Plotnikov, P.I., J.Toland, J.F.: Standing waves on an infinitely deep perfect fluid under gravity. Arch. Rat. Mech. Anal. 177, 367-478 (2005)

16. Iooss, G., Plotnikov, P.I.:Small divisor problem in the theory of threedimensional water gravity waves. Memoirs of AMS, 200, 940, (2009)

17. Kirchgässner, K.: Wave solutions of reversible systems and applications. J.Diff. Eqns. 45, 113-127 (1982)

18. Lannes, D.: Well-posedness of the water-waves equations. J.Amer. Math. Soc. $18,605-654(2005)$

19. Levi-Civita, T.: Détermination rigoureuse des ondes permanentes d'ampleur finie. Math. Annalen 93, 264-314 (1925)

20. Moser, J.: Minimal solutions of variational problems on a torus. Ann. Inst. H .Poincare Anal. Non Lineaire, 3, 229-272 (1986)

21. Moser,J.: A stability theorem for minimal foliations on a torus. Ergodic Th. \& Dynamical syst. 8*, 251-281 (1988)

22. Nekrasov, A.I.: On waves of permanent type. Izv. Ivanovo-Voznesensk. Politekhn. Inst., 3, 52-65 (1921)

23. Rabinowitz, P.H., Stredlinsky, E.: On some results of Moser and of Bangert. Ann. Inst. H. Poincaré, Anal. Non Linéaire, 21, 673-688 (2004)

24. Reeder,J., Shinbrot, M.: Three-dimensional, nonlinear wave interaction in water of constant depth. Nonlinear Anal., T.M.A., 5 , 303-323 (1981)

25. Roberts, A., Schwartz, L.: The calculation of nonlinear short-crested gravity waves. Phys. Fluids 26, 2388-2392 (1983)

26. Sretenskii, L.: Spatial problem of determination of steady waves of finite amplitude (russian). Dokl. Akad. Nauk SSSR (N.S.) 89 , 25-28 (1953)

27. Stokes, G.G.: On the theory of oscillatory waves. Trans. Camb. Phil. Soc. 8, 441-455 (1847)

28. Zakharov, V.E.: Stability of periodic waves of finite amplitude on the surface of a deep fluid. J.Appl. Mech. Tech. Phys. 9 190-194 (1968) 